PROACTT V TY AND I N ECT V TY OF HORI ZONTAL VELLS

ANUAL TECHN CAL REPORT

REPCRTI NG PER OD. 03/ 10/1996 - 03/ 09/ 1997

\author{
AUTHORS: KHALI D AZ Z \\ THOMAS A HEVETT \\ SEPEHR ARABABI \\ I SSUE DATE: 04/29/1997 \\ DE- FG22- 93BC14862- - 18 \\ STANFCRD UN VERSI TY \\ DEPARTMENT OF PEIRQLEUM ENG NEERN NG \\ STANFORD, CA 94305-2220
}




\section{Disclaimer}

This report was prepared as an account of work sponsored by an agency of the United States Government. Neither the United States Government nor any agency thereof, nor any of their employees, makes any warranty, express or implied, or assumes any legal liability or responsibility for the accuracy, completeness, or usefulness of any information, apparatus, product, or process disclosed, or represents that its use would not infringe privately owned rights. Reference herein to any specific commercial product, process, or service by trade

name, trademark, manufacturer, or otherwise does not necessarily constitute or imply its endorsement, recommendation, or favoring by the United States Government or any agency thereof. The views and opinions of authors expressed herein do not necessarily state or reflect those of the United States Government or any agency thereof. 


\section{Contents}

1 Single Phase Fluid Flow in a Wellbore $\ldots \ldots \ldots \ldots \ldots \ldots \ldots \ldots \ldots \ldots \ldots$

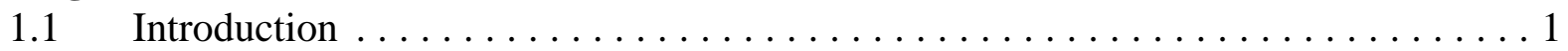

1.2 Influences of Mass Transfer through Pipe Wall $\ldots \ldots \ldots \ldots \ldots \ldots \ldots \ldots \ldots$

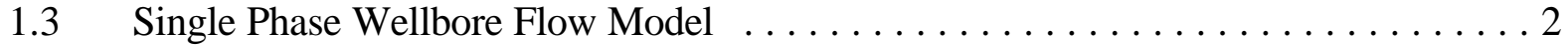

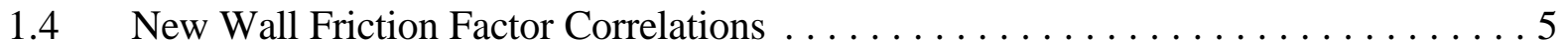

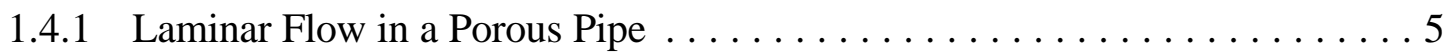

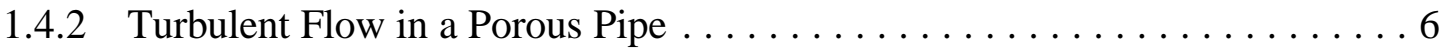

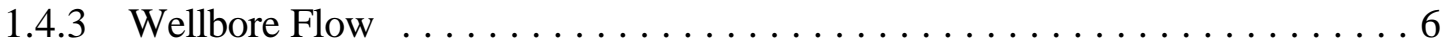

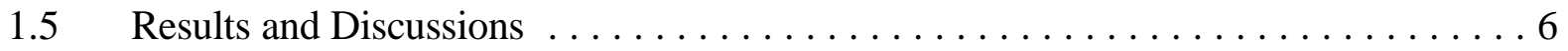

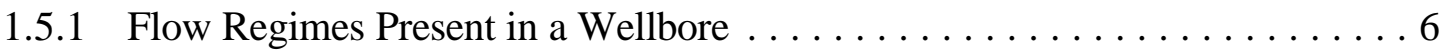

1.5.2 Partially-Perforated Horizontal Well Example . . . . . . . . . . . . . . 7

1.5.3 Fully-Perforated Horizontal Well Example . . . . . . . . . . . . . 7

1.5.4 Coupling the New Wellbore Model with a Reservoir Inflow Model . . . . . . 8

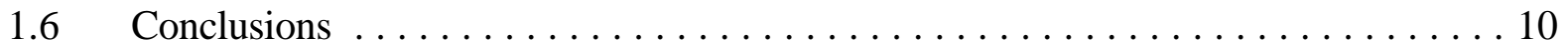

2 Development and Testing of a New Mechanistic Model for Multiphase

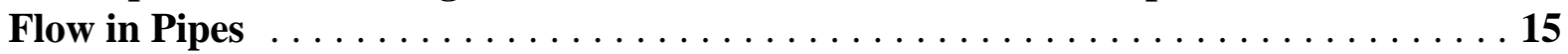

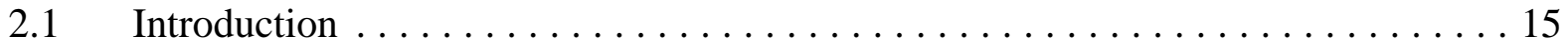

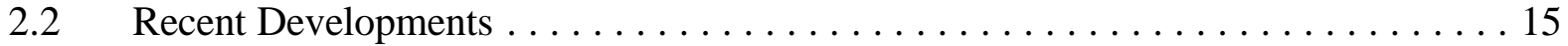

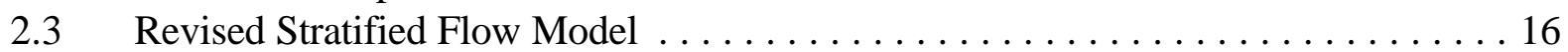

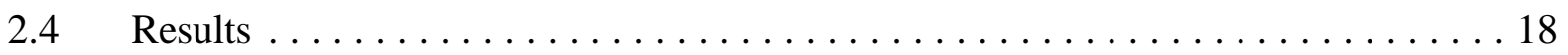

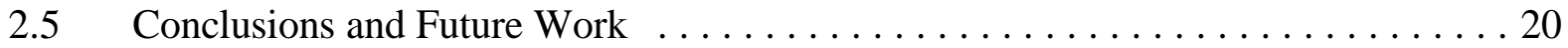

3 Analysis of the 1995 and 1996 Stanford Horizontal Wellbore Experiments $\ldots \ldots \ldots 22$

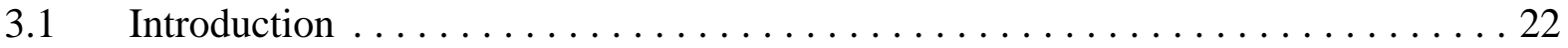

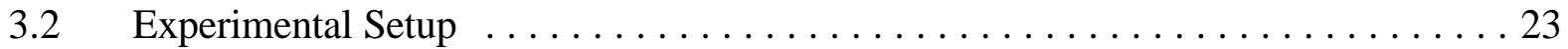

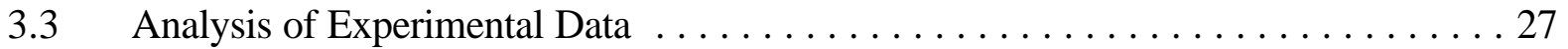

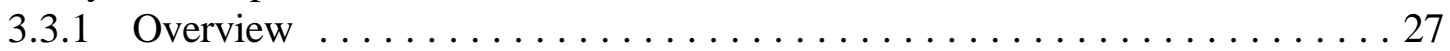

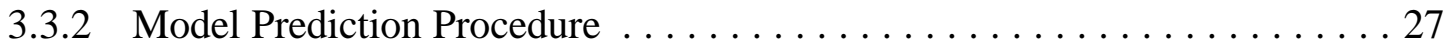

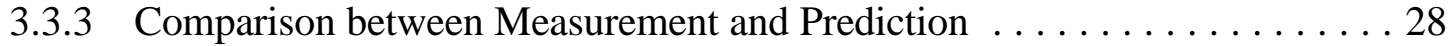

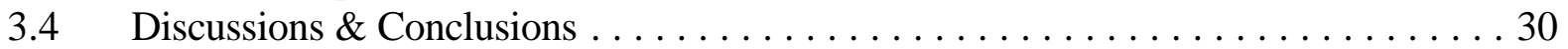

4 Effects of Pressure Drop in Horizontal Wells and Optimum Well Length . . . . . . 47

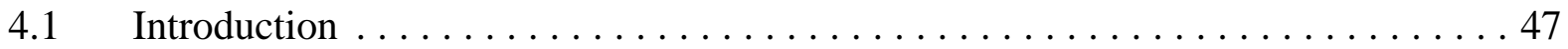

4.1 .1 Type 1: Infinitely Conductive Well . . . . . . . . . . . . . . 48

4.1.2 Type 2: Wellbore Coupled to an Analytically Approximated Reservoir . . . 48 
4.1.3 Type 3: Wellbore Coupled to a Numerically Approximated Reservoir . . . . 49

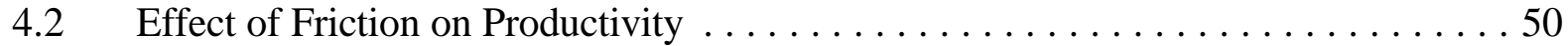

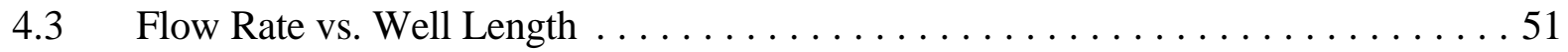

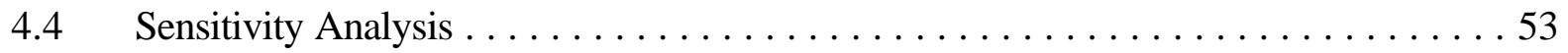

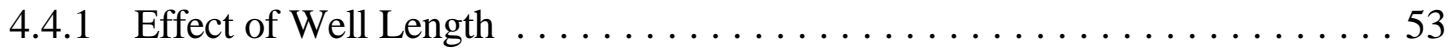

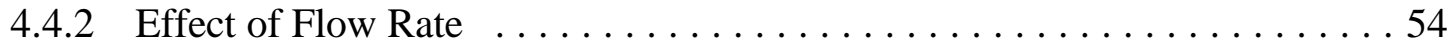

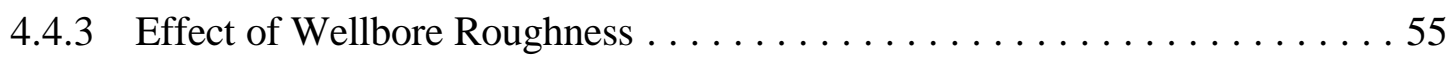

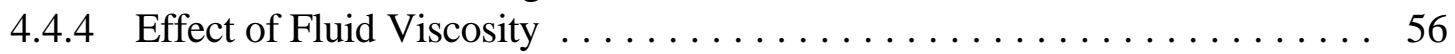

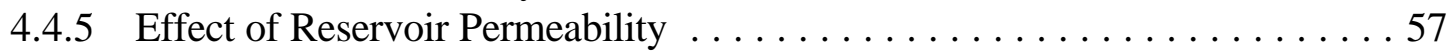

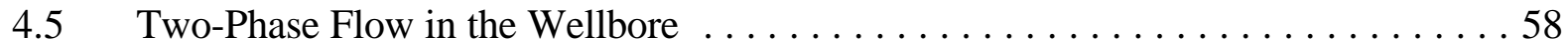

4.6 Frictional Effects on Water/Gas Breakthrough $\ldots \ldots \ldots \ldots \ldots \ldots \ldots \ldots \ldots$

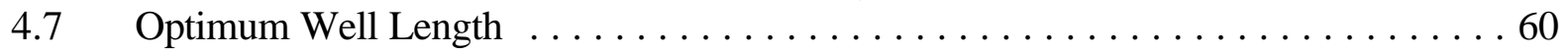

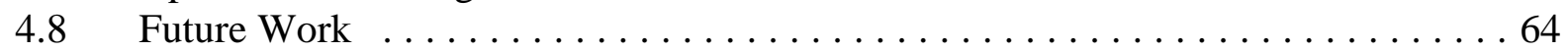

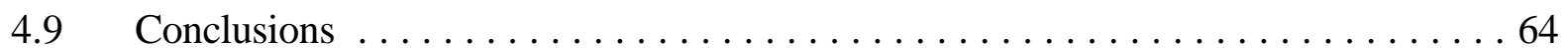

Appendix A: Flow Rate vs. Well Length $\ldots \ldots \ldots \ldots \ldots \ldots \ldots \ldots \ldots \ldots \ldots$

Appendix B: Optimum Well Length $\ldots \ldots \ldots \ldots \ldots \ldots \ldots \ldots \ldots \ldots \ldots \ldots \ldots$

5 Effects of Wellbore Friction under Transient Conditions $\ldots \ldots \ldots \ldots \ldots \ldots$

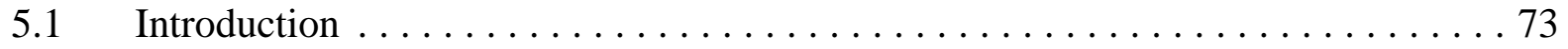

5.2 Babu \& Odeh's Solution for Horizontal Well Productivity $\ldots \ldots \ldots \ldots \ldots \ldots$

5.3 Analytical Solution: Infinite Conductive Well . . . . . . . . . . . . . 75

5.4 Frictional Pressure Drop in the Wellbore $\ldots \ldots \ldots \ldots \ldots \ldots \ldots \ldots \ldots$

6 Effects of Grid Systems on Predicting Horizontal Well Productivity $\ldots \ldots \ldots \ldots$

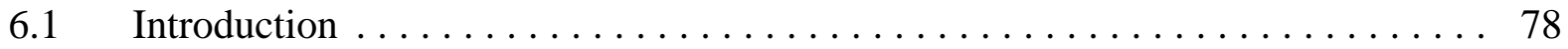

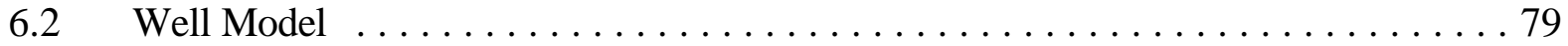

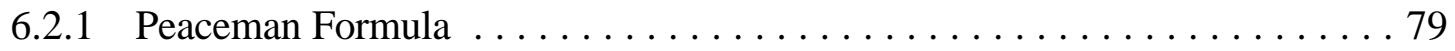

6.2 .2 Babu and Odeh's Analytical Solution $\ldots \ldots \ldots \ldots \ldots \ldots \ldots$

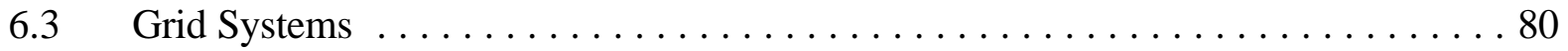

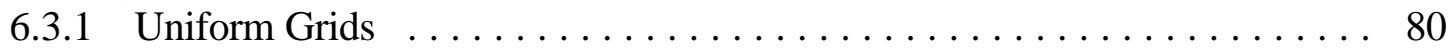

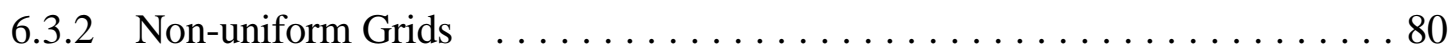

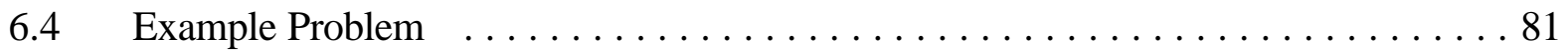

6.4 .1 Model Description $\ldots \ldots \ldots \ldots \ldots \ldots \ldots \ldots \ldots \ldots \ldots \ldots \ldots$

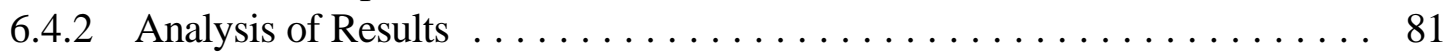

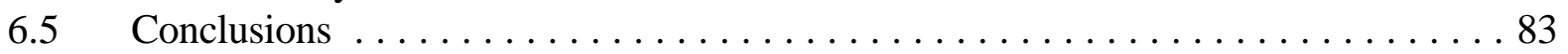

7 Effect of Reservoir Heterogeneities and Other Parameters on Performance

Prediction of Non-Conventional Wells $\ldots \ldots \ldots \ldots \ldots \ldots \ldots \ldots \ldots \ldots \ldots \ldots \ldots$

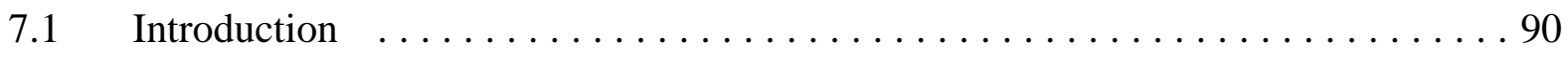

7.2 Mat is Needed for Preducting Performance $\ldots \ldots \ldots \ldots \ldots \ldots \ldots \ldots \ldots$

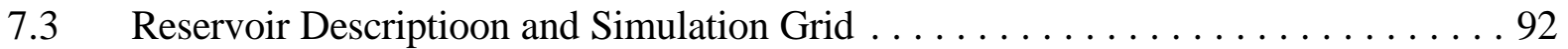

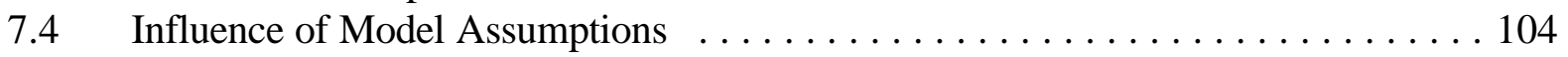

7.4 .1 Well Models . . . . . . . . . . . . . . . . . . . . . 104 
7.4.2 Effect of Wellbore Pressure Drop on Well Performance $\ldots \ldots \ldots \ldots . . . . .105$

7.4.3 Analytical Cresting Models ............................ 105

7.4.4 Productivity Models . . . . . . . . . . . . . . . . . . . . . . . . . . . . 106

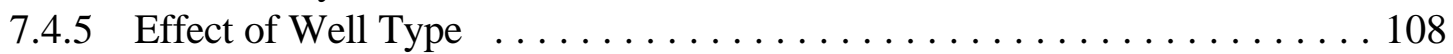

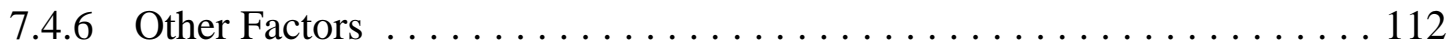

7.4.7 Concluding Remarks .............................. 112

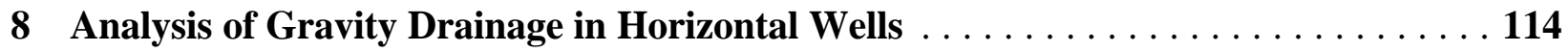

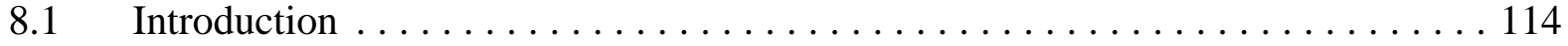

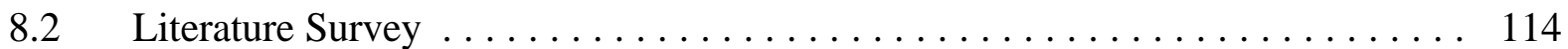

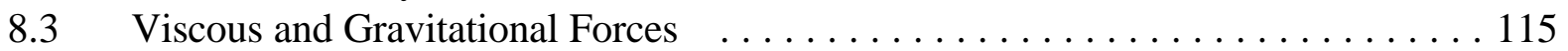

8.4 Experimental Setup and Procedure . . . . . . . . . . . . . . . . . . . . 116

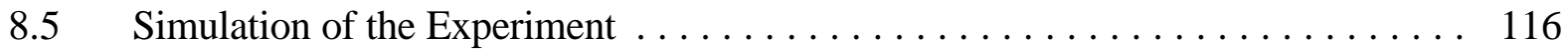

8.6 Future Work . . . . . . . . . . . . . . . . . . . . . . . . . . . . . 118

9 Correlations for Cresting Behavior in Horizontal Wells $\ldots \ldots \ldots \ldots \ldots \ldots \ldots$

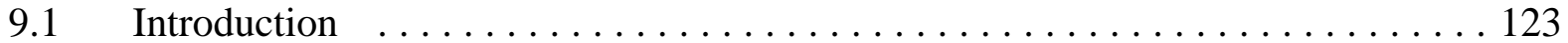

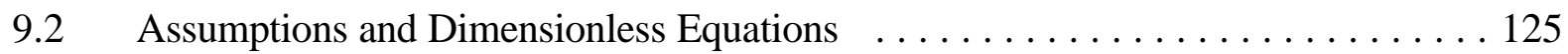

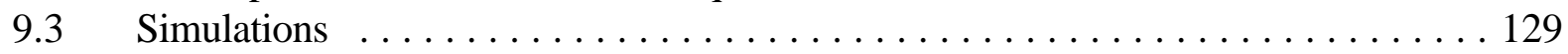

9.3.1 Grid Block Size . . . . . . . . . . . . . . . . . . . . . . . . . 129

9.3.2 Vertical Gridblock Size Analysis . . . . . . . . . . . . . . . . . . . . 131

9.3.3 Horizontal Gridblock Size Analysis . . . . . . . . . . . . . . . 131

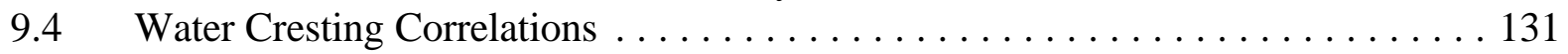

$9.4 .1 \quad$ Breakthrough Time ................................ 131

9.4.2 Post-Breakthrough Behavior . . . . . . . . . . . . . . . . . . . . . . . 140

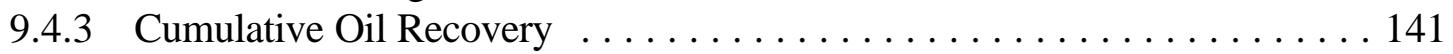

9.4 .4 Optimum Grid . . . . . . . . . . . . . . . . . . . . . . . . . 141

$9.5 \quad$ Applications ......................................... 144

9.5.1 Maximum Rate .................................... 144

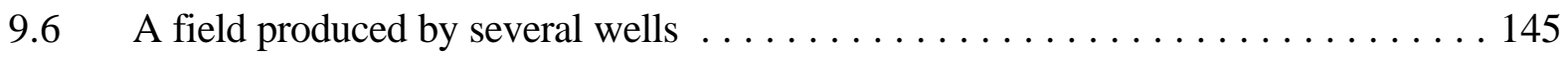

9.7 Estimation of breakthrough time . . . . . . . . . . . . . . . . . . . 146

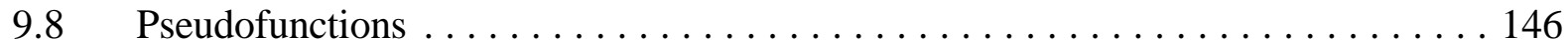

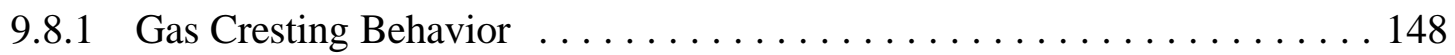

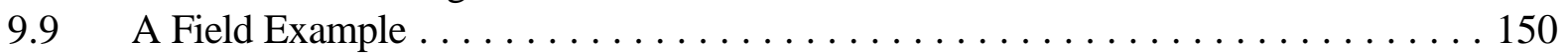

9.9.1 Water-oil flow . . . . . . . . . . . . . . . . . . . . . 153

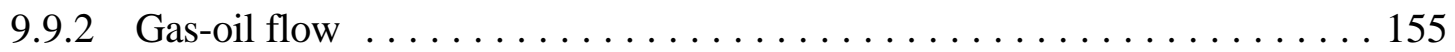

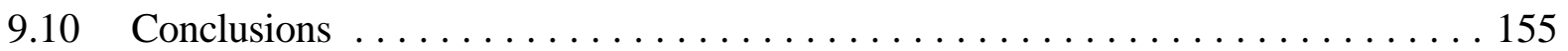

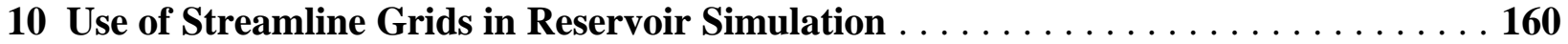

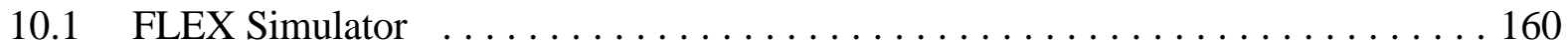

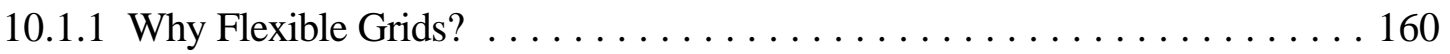

10.1 .2 Numerical Model $\ldots \ldots \ldots \ldots \ldots \ldots \ldots \ldots \ldots \ldots \ldots \ldots \ldots$

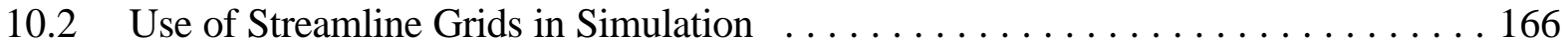

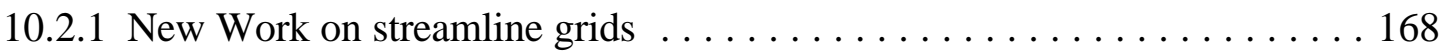

10.2.2 Results .................................... 169 


\section{List of Figures}

1.1 Mass and Momentum Transfer along a Wellbore with Mass Transfer through

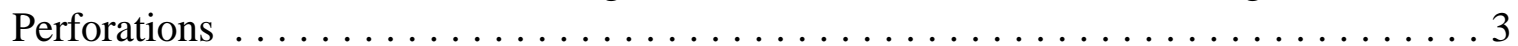

1.2 Pressure Gradients Change with Wellbore Location (well \#1) $\ldots \ldots \ldots \ldots \ldots \ldots \ldots$

1.3 Change in Cumulative Pressure Drops with Wellbore Location (well \#1) . . . . . . 8

1.4 Pressure Gradients Change with Wellbore Location (well \#2) . . . . . . . . . . . . 9

1.5 Pressure Gradients Change with Wellbore Location (well \#3) $\ldots \ldots \ldots \ldots \ldots \ldots$

1.6 Change in Cumulative Pressure Drops with Wellbore Location (well \#3) . . . . . . . 11

1.7 Specific Inflow Rate Distribution (well \#3) . . . . . . . . . . . . . . . . 12

1.8 Overall Well Production Rate Change with Wellbore Length (well \#3) . . . . . . . . . 12

2.1 Momentum Balance with Two Segregated Fluids $\ldots \ldots \ldots \ldots \ldots \ldots \ldots$

2.2 Comparison of Calculated Liquid Volume Fraction with Experimental Data . . . . . . 18

2.3 Comparison of Calculated Pressure Gradient with Experimental Data . . . . . . . . . . 19

3.1 Wellbore Section Used for the 1996 Horizontal Flow Experiments . . . . . . . . . . . . 24

3.2 Average Measured Wellbore Internal Diameter for Each Section . . . . . . . . . . . . 24

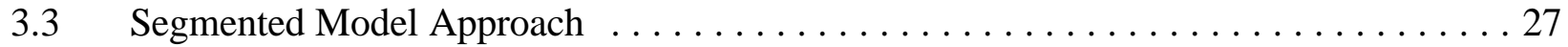

3.4 Comparison between Measured Pressure Drop and Prediction by Petalas \& Aziz (1996) Model (1995 Experiments) . . . . . . . . . . . . . . . . . . . 32

3.5 Comparison between Measured Pressure Drop and Prediction by Xiao, Shoham \& Brill (1990) Model (1995 Experiments) . . . . . . . . . . . . . . . . . . . 33

3.6 Comparison between Measured Pressure Drop and Prediction by Beggs \& Brill (1973, revised 1977) Model (1995 Experiments) . . . . . . . . . . . . . . . . 34

3.7 Comparison between Measured Pressure Drop and Prediction by Dukler, Wicks \& Cleveland (1964) Correlation (1995 Experiments) . . . . . . . . . . . . . . . . 35

3.8 Comparison between Measured Pressure Drop and Prediction by the Homo geneous Model (1995 Experiments) . . . . . . . . . . . . . . . . . . . . . 36

3.9 Comparison between Measured Pressure Drop and Prediction by Petalas \& Aziz (1996) Model (1995 Experiments) . . . . . . . . . . . . . . . . . . . . . . . 37

3.10 Comparison between Measured Pressure Drop and Prediction by Xiao, Shoham \& Brill (1990) Model (1995 Experiments) . . . . . . . . . . . . . . . . . . . . 38

3.11 Comparison between Measured Pressure Drop and Prediction by Beggs \& Brill (1973, revised 1977) (1995 Experiments) . . . . . . . . . . . . . . . . . . . . 39

3.12 Comparison between Measured Pressure Drop and Prediction by Dukler, Wicks \& Cleveland (1964) Correlation (1995 Experiments) . . . . . . . . . . . . . . . 40 
3.13 Comparison between Measured Pressure Drop and Prediction by the Homogeneous Model (1995 Experiments) . . . . . . . . . . . . . . . . . . . . . 41

3.14 Comparison between Measured Pressure Drop and Prediction by Petalas \& Aziz (1996) Model (1996 Experiments) . . . . . . . . . . . . . . . . . . . . 42

3.15 Comparison between Measured Pressure Drop and Prediction by Xiao, Shoham \& Brill (1990) Model (1996 Experiments) . . . . . . . . . . . . . . . . . . 42

3.16 Comparison between Measured Pressure Drop and Prediction by Beggs \& Brill (1973, revised 1977) Model (1996 Experiments) . . . . . . . . . . . . . . 43

3.17 Comparison between Measured Pressure Drop and Prediction by Dukler, Wicks \& Cleveland (1964) Correlation (1996 Experiments) . . . . . . . . . . . . . . 43

3.18 Comparison between Measured Pressure Drop and Prediction by the Homogeneous Model (1996 Experiments) . . . . . . . . . . . . . . . . . . . . . . . 44

3.19 Comparison between Measured Pressure Drop and Prediction by Petalas \& Aziz (1996) Model (1996 Experiments) . . . . . . . . . . . . . . . . . . . . 44

3.20 Comparison between Measured Pressure Drop and Prediction by Xiao, Shoham \& Brill (1990) Model (1996 Experiments) . . . . . . . . . . . . . . . . . . 45

3.21 Comparison between Measured Pressure Drop and Prediction by Beggs \& Brill (1973, revised 1977) Model (1996 Experiments) . . . . . . . . . . . . . . . 45

3.22 Comparison between Measured Pressure Drop and Prediction by Dukler, Wicks \& Cleveland (1964) Correlation (1996 Experiments) . . . . . . . . . . . . . . . 46

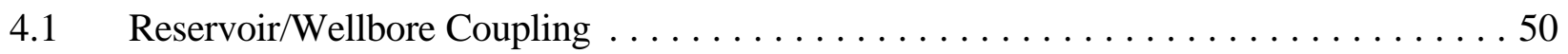

4.2 Effect of Flow Rate and Well Length on Wellbore Friction $\ldots \ldots \ldots \ldots \ldots \ldots$

4.3 Effect of Roughness on Wellbore Friction (Drawdown $=25 \mathrm{psi} \ldots \ldots \ldots \ldots \ldots \ldots$

4.4 Effect of Oil Viscosity on Wellbore Friction . . . . . . . . . . . . . . . . . 57

4.5 Effect of Reservoir Permeability on Wellbore Friction $\ldots \ldots \ldots \ldots \ldots \ldots \ldots$

4.6 Effect of Two-Phase Flow in the Well on Wellbore Friction . . . . . . . . . . . . . . 59

4.7 Early Breakthrough at the Heel of the 1 Well Due to Wellbore Friction . . . . . . . . . 60

4.8 Optimum Well Length Calculation $\ldots \ldots \ldots \ldots \ldots \ldots \ldots \ldots \ldots \ldots \ldots \ldots \ldots \ldots \ldots \ldots \ldots$

$5.1 \quad$ Horizontal well in a box-shaped reservoir (Babu and Odeh [1]) $\ldots \ldots \ldots \ldots \ldots 74$

6.1 Influence of Grid System on Production Ratio for the Case of Constant Well Pressure Constraint ................................ 85

6.2 An Expanded Portion of Figure 6.1 from 20 to 25 Days . . . . . . . . . . . 85

6.3 Approach to Pseudo Steady State for the Case of Constant Well Pressure

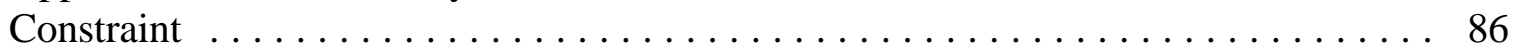

6.4 Adjustment Factor for WI to obtain Correct Flow Rate - Isotropic Case . . . . . . . . 86

6.5 Approach to Pseudo Steady State for the Constant Flow Rate Case . . . . . . . . . . . 87

6.6 Results of Constant Flow Rate Set Initially Followed by a Period of Constant Wellbore Pressure Constraint . . . . . . . . . . . . . . . . . . . . 87

6.7 Bottomhole Pressure in the Case of Constant Flow Rate Constraint . . . . . . . . . 88

6.8 An Expanded Portion of Figure 6.7 from 15 to 20 Days . . . . . . . . . . . 88

6.9 Variation of Oil Rate with Well Index for both Isotropic and Anisotropic Cases . . . . . 89 
6.10 Variation of Bottomhole Pressure with Well Index for both Isotropic and Anisotropic Case ............................................ 89

7.1 Histograms of Horizontal and Vertical Permeability for Fine, Medium, and Coarse Resolutions . ................................. 95

7.2 Horizontal and Vertical Permeability Maps of Realization 3 for Fine, Mediumf, Coarse-f, and Medium-p Grids . . . . . . . . . . . . . . . . . 96

7.3 Horizontal and Vertical Permeability Maps of Realization 9 for Fine, Mediumf, Coarse-f, and Medium-p Grids . . . . . . . . . . . . . . . . . . . . . . . 97

7.4 Horizontal and Vertical Permeability Maps of Realization 12 for Fine, Mediumf, Coarse-f, and Medium-p Grids . . . . . . . . . . . . . . . . . . . 98

7.5 Horizontal and Vertical Permeability Maps of Realization 19 (Base Case) for Fine, Medium-f, Coarse-f, and Medium-p Grids . . . . . . . . . . . . . . . . 99

7.6 Oil Production Rate for 20 Realizations on Medium-f Grid . . . . . . . . . . . . . . 100

$7.7 \quad$ GOR for 20 Realizations on Medium-f Grid . . . . . . . . . . . . . . . . . . . . . 100

$7.8 \quad$ WOR for 20 Realizations on Medium-f Grid ..................... 101

7.9 Comparison of Oil Production Rates from Different Grids for the Base Case (Realization 19) .................................... 101

7.10 Comparison of Gas/Oil Ratio (GOR)from Different Grids for the Base Case

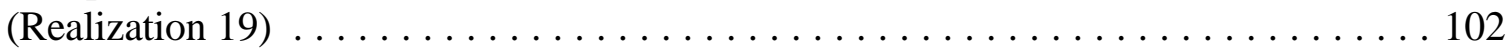

7.11 Comparison of Water/Oil Ratio (WOR)from Different Grids for the Base Case

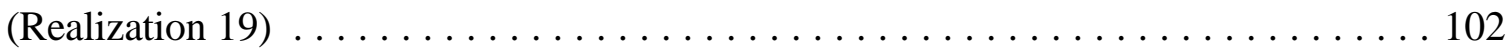

7.12 Effect of Well Index (WI) on Oil Production Rate and GOR for the Base Case (Realization 19) ................................... 105

7.13 Comparison Between Singl Phase (Analytical and Simulation) and ThreePhase Medium Grid Simulation with Homogeneous Properties . . . . . . . . . . . . 107

7.14 Oil Production Rate for 20 Realizations on Medium-f Grid for Vertical Well

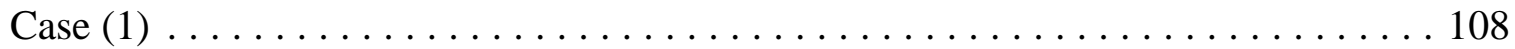

7.15 GOR for 20 Realizations on Medium-f Grid for Vertical Well Case (1) . . . . . . . . 109

7.16 WOR for 20 Realizations on Medium-f Grid for Vertical Well Case (1) . . . . . . . . 109

7.17 Oil Production Rate for 20 Realizations on Medium-f Grid for Vertical Well Case (1) ......................................... 110

7.18 GOR for 20 Realizations on Medium-f Grid for Vertical Well Case (2) . . . . . . . 111

7.19 GOR for 20 Realizations on Medium-f Grid for Vertical Well Case (2) . . . . . . . 111

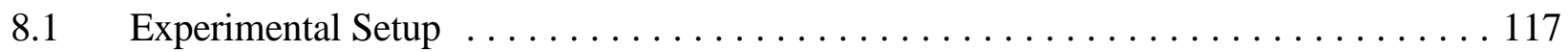

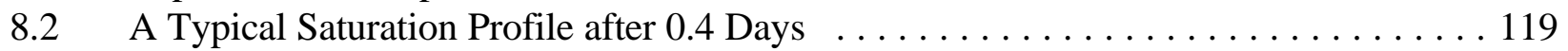

8.3 Production Rate of Oil versus Time for Parallel Horizontal Injector and Producer . . 119

8.4 Saturation Profile in X-Z Direction for Horizontal Injector and Producer . . . . . . . 120

8.5 Saturation Profile in X-Z Direction for Vertical Injector and Producer . . . . . . . . 120

8.6 Oil Production Rate for Vertical Injector and Producer $\ldots \ldots \ldots \ldots \ldots \ldots \ldots \ldots$

8.7 Gas Production Rate for Vertical Injector and Producer . . . . . . . . . . . . . . . 121

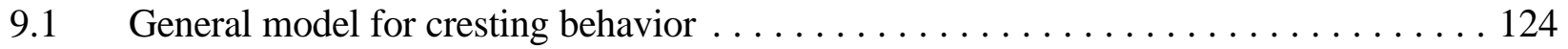

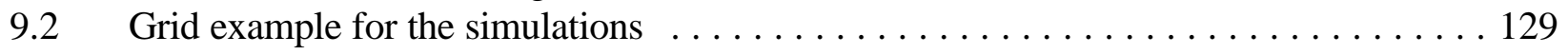


9.3 Effect of number of blocks used between the top of the formation and water-oil

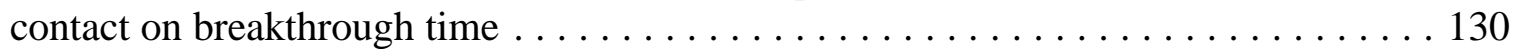

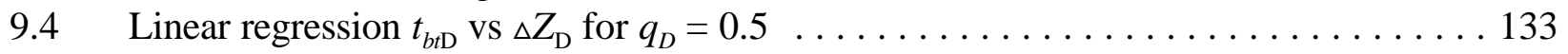

9.5 Comparison of breakthrough time and $q_{o D}$ correlations with simulation results

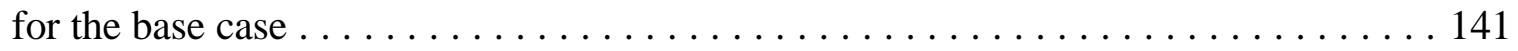

9.6 Comparison of breakthrough time and WOR correlations with simulation results for the base case . . . . . . . . . . . . . . . . . . . 142

9.7 Comparison of $N_{p D} v s t_{D}$ between the correlations and the simulation results

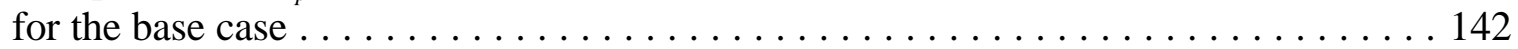

9.8 Effect of vertical gridblock size on WOR as a function of time $\ldots \ldots \ldots \ldots \ldots \ldots$

9.9 Evenly spaced wells in a field with a specified total rate $\ldots \ldots \ldots \ldots \ldots \ldots$

$9.10 \quad N_{p D} v s t_{D}$ curves for a field with four wells $\ldots \ldots \ldots \ldots \ldots \ldots \ldots \ldots \ldots \ldots \ldots \ldots$

9.11 Extrapolation of two coarse grid simulations to find $t_{b t D}^{0} \quad \ldots \ldots \ldots \ldots \ldots \ldots \ldots$

9.12 Procedure to obtain $n_{o p}$ for a given $n_{o}$ and $\Delta Z_{D} \ldots \ldots \ldots \ldots \ldots \ldots \ldots \ldots \ldots$

9.13 Application of pseudo $n_{o}$ for the base case data . . . . . . . . . . . . . 149

9.14 Variation of $n_{o}, n_{w}$ or $M$ to generate pseudofunctions for any simulation . . . . . . 149

9.15 Comparison of breakthrough time and GOR from correlations with simulation

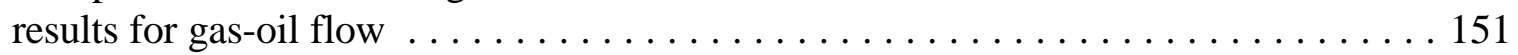

9.16 Comparison of $N_{p D}$ calculated from correlations to simulation results for gas-

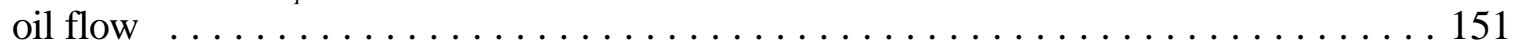

9.17 Comparison of breakthrough time and WOR correlations with simulation re-

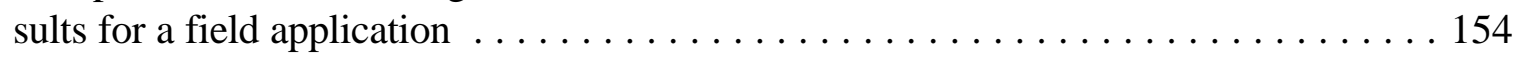

9.18 Comparison of breakthrough time and GOR correlations with simulation re-

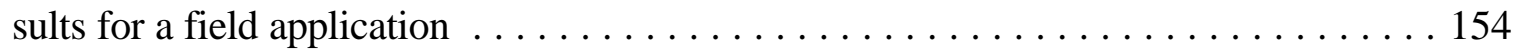

10.1 Modules Used for Grid Generation in 2D (After Ref [1], Chapter 4) . . . . . . . 161

10.2 A 2D Voronoi Grid Using Modules (After Ref [1], Chapter 4) . . . . . . . . . . . 162

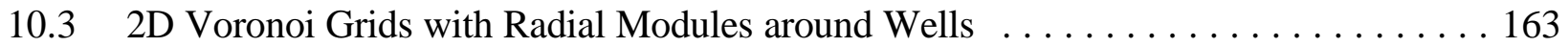

10.4 2D CVFE Grid with Radial Modules around Wells . . . . . . . . . . . . . . . . 164

10.5 Example of a Control Volume and its Connections to the other Grid Blocks . . . . . . 165

10.6 Streamline Grid Generated after Selecting Some Streamlines and Upscaling Permeabilities . . . . . . . . . . . . . . . . . . . . . . . . . 167

10.7 Streamlines generated by Injecting at the Most Left Boundary and Producing at the Most Right Boundary . . . . . . . . . . . . . . . . . . . 167

10.8 Construction of Grid Blocks Using Streamlines and Equipotentials . . . . . . . . . . . 168

10.9 Locations of Pressure Points for Interpolation and Transmissibility Calculations . . . . 169

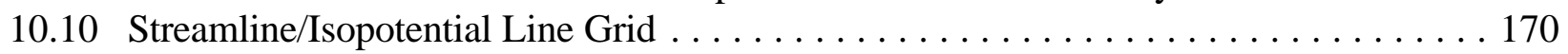

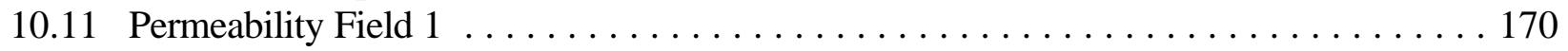

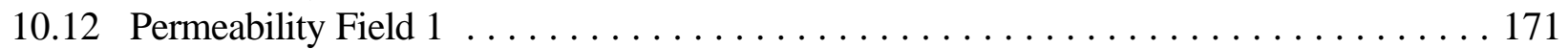

10.13 Three Different Grids Corresponding to the Permeability Field $1 \ldots \ldots$. . . . . . 172

10.14 Water-Cut Response for the Three Grids Used in CASE $1 \ldots \ldots \ldots \ldots \ldots$

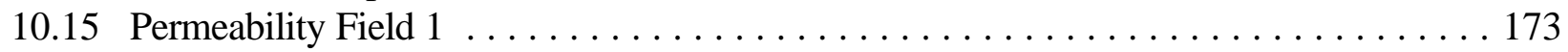

10.16 Three Different Grids Corresponding to the CASE $2 \ldots \ldots \ldots \ldots \ldots \ldots \ldots$

10.17 Water-Cut Response for the Three Grids Used in CASE $2 \ldots \ldots \ldots \ldots \ldots$ 


\section{List of Tables}

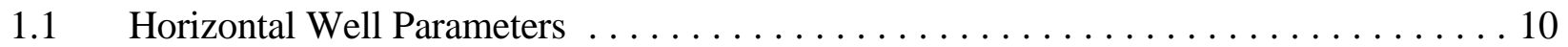

Flow Pattern Distribution in Experimental Data $\ldots \ldots \ldots \ldots \ldots \ldots$

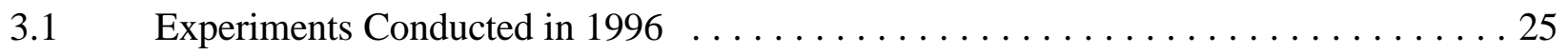

4.1 Parameters Used in Example Problem $\ldots \ldots \ldots \ldots \ldots \ldots \ldots \ldots \ldots \ldots \ldots \ldots$

Reservoir and Fluid Properties $\ldots \ldots \ldots \ldots \ldots \ldots \ldots \ldots \ldots \ldots \ldots \ldots \ldots \ldots$

7.1 Summary of Rock and Fluid Properties Used $\ldots \ldots \ldots \ldots \ldots \ldots$

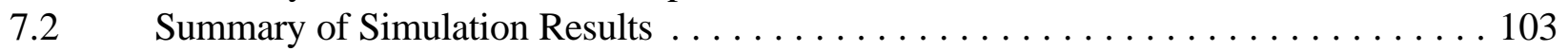

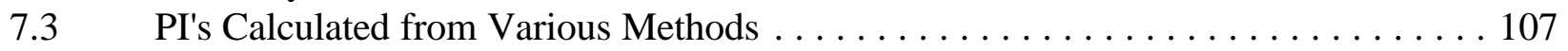

9.1 Base case data set for simulation runs $\ldots \ldots \ldots \ldots \ldots \ldots \ldots \ldots \ldots \ldots \ldots \ldots$

9.2 Base case for the simulations and range of variation of each dimensionless

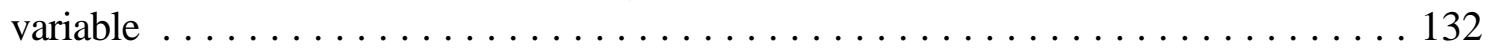

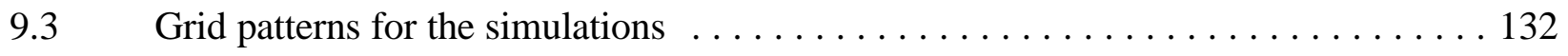

9.4 Values of $t^{o}{ }_{b t D}$, $\alpha$ for breakthrough time and $m$ for $q_{o D}$ for each dimensionless variable simulated. A blank entry indicates that dimensionless variable has

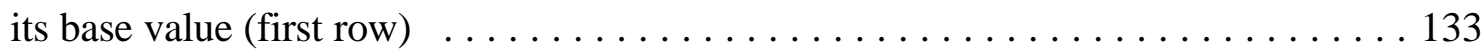

9.5 Values of for breakthrough time and $m$ for $q_{o D}$ for simultaneous variations of $q_{D}$ with $M$ or $\varkappa_{e D} . H_{w D}, n_{o}$ and $n_{w}$ are all equal to 1 , their base values. Also a blank entry indicates that dimensionless variable has its base

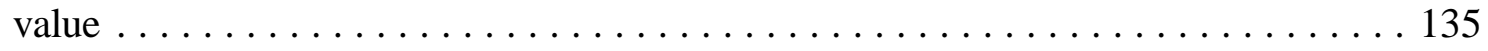

9.6 Values of $t_{b t D}^{o}, \alpha$ for breakthrough time and $m$ for $q_{o D}$ for simultaneous variations of $q_{D}$ with $n_{o} . x_{e D}=20$ and $\mathrm{M}, h_{w D}$, and $n_{o}$ are equal to 1 , their base

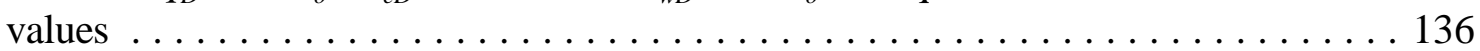

9.7 Equations and functions for the correlations of $t_{b t D}^{o}, \alpha$ and $m\left(q_{o D}\right) \ldots \ldots \ldots$

9.8 Values of the constants for the correlations of $t_{b t D}^{o}, \alpha$ and $m\left(q_{o D}\right)$. A - means that the constant does not appear in the equation for that factor $\ldots \ldots \ldots \ldots 139$

9.9 Variations in breakthrough time and $N_{p D}$ with vertical gridblock size for the

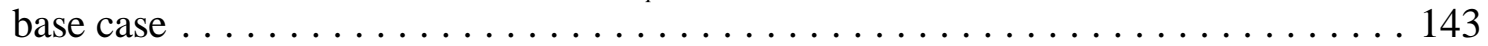

9.10 Field data set used to apply the developed correlations $\ldots \ldots \ldots \ldots \ldots \ldots 151$

9.11 Water-oil relative permeability curves for field data $\ldots \ldots \ldots \ldots \ldots \ldots \ldots$

9.12 Gas-oil relative permeability curves for field data $\ldots \ldots \ldots \ldots \ldots \ldots \ldots \ldots \ldots$

9.13 Dimensionless variables for water-oil flow for the field data . . . . . . . . . . 152

9.14 Dimensionless variables for gas-oil flow for real field data $\ldots \ldots \ldots \ldots \ldots \ldots$ 


\section{Disclaimer}

This report was prepared as an account of work sponsored by an agency of the United States Government. Neither the United States Government nor any agency thereof, nor any of their employees, makes any warranty, express or implied, or assumes any legal liability or responsibility for the accuracy, completeness, or usefulness of any information, apparatus, product, or process disclosed, or represents that its use would not infringe privately owned rights. Reference herein to any specific commercial product, process, or service by trade name, trademark, manufacturer, or otherwise does not necessarily constitute or imply its endorsement, recommendation, or favoring by the United States Government or any agency thereof. The views and opinions of authors expressed herein do not necessarily state or reflect those of the United States Government or any agency thereof. 


\title{
Management Summary
}

\section{DOE Approval}

The DOE approval for the annual renewal of the research grant to the Stanford Project on the Productivity and Injectivity of Horizontal Wells was received in early March 1996. The Project formally commenced on March 10, 1993. Mr. Thomas Reid is the DOE Project Manager in Bartlesville and Mr. John Augustine is the DOE Contracts Officer in Pittsburgh.

\section{Industrial Affiliates Program}

The DOE Project operates in association with an Industrial Affiliates program on horizontal wells, for which oil company membership has also continued during 1996. The membership during this year comprised the following organizations:

\author{
Amoco (USA) \\ AGIP (Italy) \\ ARTEP/IFP (France) \\ BP Exploration (USA) \\ Chevron (USA) \\ INTEVEP (Venezuela) \\ Marathon (USA) \\ Mobil (USA) \\ Norsk Hydro (Norway) \\ Petrobras America (Brazil) \\ Phillips Petroleum (USA) \\ Texaco (USA) \\ Union Pacific Resources (USA) \\ US Department of Interior/ \\ Minerals Management Service (USA)
}

\section{Project Goals}

The Project has eight principal goals to be studied and developed over a five year period. These goals are as follows:

TASK 1: Advanced Modeling of Horizontal Wells - Develop special gridding techniques and associated averaging algorithms for accurate simulation of HW-performance.

TASK 2: Investigate and Incorporate the Effects of Reservoir Heterogeneities - Study impacts of various types of heterogeneity and develop methods for incorporating their effects in both fine grid and coarse grid models.

TASK 3: Develop Improved Methods for Calculating Multi-Phase Pressure Drops within the Wellbore - Plan, execute, and interpret two-phase flow experiments at an oil company research facility, and use results to analyze/validate a new two-phase model. 
TASK 4: Pseudo-Functions - Define improved methods for computing two-phase pseudofunctions for effective relative permeabilities for coarse grid blocks near an HW - determine sensitivities to heterogeneities, flow conditions, skin factors, etc.

TASK 5: Develop Multi-Well Models - Develop numerical techniques and software in a parallel computing architecture capable of interactively coupling multiple detailed HWmodels to a large scale reservoir simulator.

TASK 6: Test HW-Models with Field Examples - Work with affiliate's member companies to establish HW-modeling capabilities from field measurements, particularly for pathological problem cases.

TASK 7: EOR Applications - Provide and implement practical HW aspects into modeling of EOR processes - miscible gas, steam displacement, in-situ combustion.

TASK 8: Application Studies and Their Optimization - Seek field opportunities for HW's and study their best implementation in various reservoir scenarios e.g., multiple laterals, hydraulic fracture variants, etc.

Tasks 1, 2, 3, 4, and 7 were the primary foci of activities in 1996, and this annual report is therefore written around these five tasks, which are also ongoing in 1997. Some of our principal achievements are summarized below.

\section{General Single Phase Wellbore Flow Model (Chapter 1)}

A general wellbore flow model, which incorporates not only frictional, accelerational, and gravitational pressure drops, but also the pressure drop caused by inflow for single phase flow was developed in 1996. The new wellbore model clearly identifies the relative magnitude of each pressure drop component and is readily applicable to different wellbore perforation patterns and well completions. It is found that the influence of either inflow (production well) or outflow (injection well) depends on the flow regime present in the wellbore. For laminar flow, wall friction increases due to inflow but decreases due to outflow. For turbulent flow, inflow reduces the wall friction, while outflow increases the wall friction. New wall friction correlations for wellbore flows based on our 1995 experiments have been developed.

\section{Large-Scale Flow Loop Experiments and Data Analysis (Chapters 2 and 3)}

Two-phase flow experiments were continued during the period 1995-96 in the large Wellbore Model at the Marathon Technology Center in Littleton, Colorado. Extensive modifications were made to the rig in order to (1) use 4.5 inch ID pre-packed wire wrapped screen liner (2) measure in situ liquid fractions at three window locations using nuclear densitometers, and (3) enhance the data acquisition system. The inflow section of the model was changed from $75 \mathrm{ft}$ to $66 \mathrm{ft}$ to allow for three transparent windows at which liquid fractions were measured and flow regimes were recorded. A slightly higher gas rate of $1.8 \mathrm{MMscfD}$ was achieved as opposed to $1.7 \mathrm{MMscfD}$ in 1995. The fluids in all the experiments were water and air. After running a few baseline single phase flow experiments, a total of more than 200 two-phase flow experiments were performed. These experiments included single phase axial water flow with the inflow of water or air, two phase core flow with no radial 
influx, two-phase core flow with the inflow of water or air, and finally two-phase core flow with various arrangement of the influx of water and air through perforations. In addition to horizontal flow experiments, some of the experiments were also carried out at $+2^{\circ}$ (upflow) and $-2^{\circ}$ (downflow). Differential pressures were recorded along the wellbore using Rosemount pressure transmitters. The observed flow regimes as seen through the transparent acrylic windows were recorded on video tape for every experiment. These video images will be used to check the physical flow regimes in the system with screens and compare them with the 1995 experiments where we did not have the liner. The experimental work was financed by the Industrial Consortium for this Project.

Analyses of all the 1995 and 1996 experimental data were performed. Results indicate that inflow alters flow pattern transitions significantly enough so that predictions based on available multiphase flow models could be badly in error. This indicates the need to incorporate the effects of inflow in the new Stanford mechanistic model. Theoretical efforts to accomplish this for each flow regime have already started and will continue during 1997. Advances have been made in the current mechanistic model (with no inflow). The previously developed correlations for the stratified and annular mist flow regimes have been optimized based on the available data in the Stanford Multiphase Flow Database (SMFD). The existing two-phase flow correlations do not contain features designed to represent effects of perpendicular inflow through perforations, and future work will need to include ways to handle these cross-flow perturbations. Various improvements have also been made to the many features of the SMFD.

\section{Effect of Pressure Drop and Optimum Well Length (Chapter 4)}

To understand the effect of pressure drop on cumulative oil production, a new project on the effect of friction on well productivity was launched in 1995. A semi-analytical model has been developed to evaluate horizontal well productivity with consideration to friction in the wellbore. The model involves coupling Darcy flow in the reservoir to the flow in the wellbore using material balance equations. Results obtained for the single phase oil case indicate that wellbore pressure drop becomes important only when high permeability or high production rates are involved. Both laminar and turbulent flows are considered. By combining the resulting solutions with a simple economic model, a methodology for computing the optimum well length is developed. For laminar flow, an analytical expression is derived for the optimum well length while for the more practical case of turbulent flow, an iterative procedure yields the optimum well length. The code was written in $\mathrm{C}++$, which inherently supports modularity, and is flexible enough to take advantage of any existing

or new multiphase flow models, like the one being developed under Task 3. This kind of coupling with a simulator will allow us to study the effects of wellbore pressure drop in complex heterogeneous reservoirs. Initially the well module will be linked to the reservoir simulator in an explicit fashion so that it will not adversely effect the Jacobian matrix of the simulator. In the final phase of this study, the above mentioned well module will be extended to include multi-lateral wellbores as discussed under the previous task. This comprehensive project will continue in 1997 and beyond. 


\section{Well Indices (Chapters 5 and 6)}

Previously we have shown that the correct well index for a typical partially penetrating horizontal well could be very different from the classical Peaceman well index. The correct well index can be calculated in a semi analytical manner in which the well pressure is obtained analytically and the well block pressure is computed numerically. Another approach is to eliminate the need to calculate any well index. In this so called explicit modeling, the well is very finely gridded. We studied a published utilization of this approach and compared its results with fine grid and analytical results. It is shown that this approach gives incorrect results when used with nonuniform grids having high aspect ratios.

\section{Effects of Heterogeneities on Prediction Performance (Chapter 7)}

In 1996, we also conducted a study in which we explored the reasons why performance prediction of horizontal wells is so difficult. We generated twenty consistent geostatistical descriptions of permeability and porosity that were all constrained to the hard data obtained from a vertical well previously drilled and cored. All stochastic images were created on a $100 \times 50 \times 30$ fine grid. Two sets of upscaled images of the fine grid: a $20 \times 10 \times 10$ medium grid and a $10 \times 5 \times 5$ coarse grid were also generated. The upscaling was done using two commonly used methods namely, flow-based and power-averaged. Different geological descriptions, grids and methods of upscaling gave very different results. Also we showed that many of the analytical techniques published in the literature do not provide reliable predictions.

The effect of other assumptions on performance prediction of horizontal wells such as: well index, pressure drop, analytical cresting models, and productivity models were also investigated. It is concluded that the greatest source of uncertainty is reservoir description and how it is used in simulators. Upscaling introduces errors and the scale at which upscaling is robust is not known in general. Uncertainties in the well index can cause errors in predications that are of the same magnitude as those caused by reservoir heterogeneities.

\section{Specification for EOR Extensions (Chapter 8)}

In a recently completed study of the Project in conjunction with the SUPRI-C (Gas Displacement Project at Stanford), sweep efficiencies of many well configurations were studied in a gas injection process by simulation. Comparisons were made at different viscous to gravity ratios as a way to present the effects of gravity on sweep efficiency. It was found that fully penetrating horizontal wells deliver the highest recovery among the seven different configurations studied and their best recovery is at a viscous to gravity ratio of one. For the heterogeneous systems, horizontal wells still delivered the highest recovery but this time recovery improved as the viscous to gravity ratio decreased. For vertical wells, there are severe rate limitations to achieve the gravity drainage benefits, but the use of longer horizontal wells may allow this new technique to become economically attractive. Initial simulation runs have been made to investigate the comparative aspects of sweep efficiency for vertical and horizontal wells. In this sensitivity study, the effects of many parameters such as length and location of both well types on sweep efficiency are being examined. In conjunction with these simulation runs, we are setting up laboratory experiments with horizontal wells and analog three-phase fluids to study vertical sweep, viscous fingering, and local displacement 
efficiencies. The newly installed high resolution X-ray CT Scanner with its dual X-ray energy features at Stanford will be used to monitor saturation distributions in these experiments. The experiments will be analyzed using appropriate 3D-simulation methods.

\section{Cresting Correlations and Pseudo-Functions (Chapter 9)}

Accurate representation of cresting behavior requires fine grid simulations which are costly and not always practical. This issue has been addressed by developing a coarse grid method to accurately predict breakthrough time and post breakthrough performance of horizontal wells. Correlations for breakthrough time, maximum rate, and water/oil or gas/oil ratio have been developed. The correlations are based on numerous simulations runs and some analytical approaches using an appropriate set of dimensionless variables. A procedure to derive pseudo functions either using numerical correlations or coarse grid simulations was also devised. In 1997, we plan to apply the developed correlations to various example problems including case studies using real field data.

\section{Generalized Gridding Methods (Chapter 10)}

In our flexible grid simulator, FLEX, one can align gridblock boundaries along streamlines. Examples of this technique have shown improvements in water-cut response for $2 \mathrm{D}$ areal problems. This improvement was achieved in spite of using vertical isopotential lines. In another recently developed code, this restriction has been removed. The calculated streamlines and actual isopotential lines from this new code are used to align the grids in FLEX. Advantages of this approach are being investigated.

\section{Newly Launched Projects}

A number of new projects have been recently started. Here, we only introduce them and leave the reporting on them to a later time when reasonable advance has been made in each;

- Optimal Positioning of Multi-Lateral Wells

A simulation study to investigate the productivity of multi-lateral wells in terms of their arrangement and positioning.

- Gas Condensate Reservoirs

A critical evaluation of literature has been made and the underlying equations have been derived. Both black oil and compositional formulations were considered. Derivations have revealed subtle points in the validity of some assumptions in the previous methods.

\section{- A Field Case Study}

We are in the process of obtaining data on the Dos Cuadras field from DOI/MMS. This is an offshore field located in the Eastern Santa Barbara Basin in California. We plan to build a geostatistical model for a part of the field and perform flow simulations. There are several horizontal wells in the field. We will test our predictive techniques by using data on these wells. 


\section{Affiliates Progress Review Meeting}

An annual review meeting for member companies in the Stanford Horizontal Well Project was held at Stanford on October 10-11, 1996. This meeting was well attended and member companies made presentations on their horizontal well interests and activities on the second day. The next meeting is scheduled for October 27 and 28, 1997.

\section{Papers and Presentations at Conferences, etc.}

Three papers were presented:

1. "General Wellbore Flow Model for Horizontal, Vertical, and Slanted Well Completions" presented at the 1996 SPE Annual Fall Meeting - Denver, October 6-9

2. "Why is it so Difficult to Predict the Performance of Non-Conventional Wells" presented at the 1996 SPE International Conference on Horizontal Well Technology Calgary, November 18-20

3. "Effects of Pressure Drop in Horizontal Wells and Optimum Well Length" presented at the 1997 SPE Production Operation Symposium - Oklahoma City, March 9-11

We held a joint meeting with SUPRI-A (Heavy Oil) on February 12, 1997 in Bakersfield, California to discuss research directions for a project on the use of horizontal wells in thermal recovery of heavy oils. The meeting was well attended by the Affiliate Members of Stanford groups and other interested parties. A number of possible research topics were proposed. We intend to develop several projects dealing with the application of horizontal well technology to heavy oil reservoirs.

We also organized a short course on Reservoir Simulation at Stanford University and offered it free to members of our Industrial Affiliates (July 22-26, 1996)

We also attended the following meetings:

- "Forecasting Performance of Petroleum Reservoirs: is the Answer Woth the Cost," Deans Distinguished Lecture Series, Rice University - Houston, January 31, 1997.

- "Multiphase Flow in Pipes" University of Texas, Austin, March 18, 1996.

- Lectures for Norsk Hydro, Norway, on various problems of modeling well performance, May 27 to July 7, 1996.

- Lectures for RIPED, China, on various problems of modeling well performance, July 10 to $12,1996$.

- Lectures for JNOC and JAPEX, Japan, on various problems of modeling well performance, July 15 abd 16, 1996. 


\title{
1. Single Phase Fluid Flow in a Wellbore
}

\author{
by Liang-Biao Ouyang, Sepehr Arbabi, and Khalid Aziz
}

\begin{abstract}
A general wellbore flow model is presented to incorporate not only frictional, accelerational and gravitational pressure drops, but also the pressure drop caused by inflow. Influence of inflow or outflow on the wellbore pressure drop is analyzed. New friction factor correlations accounting for both inflow and outflow are also developed.
\end{abstract}

\section{$1.1 \quad$ Introduction}

Starting with Yuan \& Finkelstein in 1956, a number of mechanical engineers have studied single phase fluid flow in porous pipes with mass transfer through the pipe wall. However, This type of flow did not interest petroleum engineers until the horizontal well technology was introduced and widely applied in the oil and gas industry in the 1980s. For fluid flow in horizontal wells, the flow format is quite similar to pipe flow with mass transfer through porous walls. The main differences between these two types of flow are as follows (Ouyang et al., [1]):

- In horizontal wells, the mass transfer is normally through perforations, while in the case of pipe flow it is through pores in the wall. In other words, the effective perforation density is very large (theoretically infinite) for the porous pipe flow case. Nevertheless, in the case of openhole completions, the horizontal well and porous pipe flow problems are conceptually identical.

- The injection rates are usually quite small in the porous pipe flow case, this is not necessarily the case for wellbore flow.

- When there is no mass transfer through the pipe wall, the effective pipe roughness is very different from the actual pipe roughness in a horizontal well due to the effect of perforations on the axial flow (such as flow separation, cavity flow or secondary flow), but it changes only slightly from the actual value for the porous pipe flow case.

Little information is available related to the significance of the above-mentioned differences on the prediction of horizontal well behavior. Although much research work has been completed for the porous pipe flow, these results may not be easily applicable to horizontal wells. Recognizing this fact, petroleum engineers have begun to study the horizontal well flow behavior since the late 1980s. Research efforts are under way for wellbore flow with mass transfer through pipe wall, but more needs to be done due to the following observations:

- No correlations exist for determining the wall friction factors for single phase and multiphase fluid flow in a wellbore with inflow or outflow through perforations. 
- The accelerational and inflow-directional pressure drop are neglected in most of the single wellbore flow models or wellbore-reservoir coupling models.

- The wall friction shears are usually evaluated by using the friction factor correlations for pipe flow without mass transfer through pipe wall. Therefore, the impact of mass transfer through pipe wall on the wall friction is not included.

- No mechanistic model has been developed to predict the flow characteristics, such as flow pattern, liquid holdup and pressure drop in multiphase wellbore flow with the presence of inflow or outflow through perforations.

\subsection{Influences of Mass Transfer through Pipe Wall}

Based on the analysis by Ouyang et al. [1,2], the influence of mass transfer through pipe wall on velocity profile and wall friction can be summarized as follows:

- Laminar Flow: when mass transfer through the pipe wall exists, the parabolic velocity profile is inappropriate for describing the velocity distribution over the pipe cross section. The velocity should increase for inflow (injection) and decrease for outflow (suction) following the law of mass conservation. Although inflow leads to the increasing of axial velocities across the whole pipe section, as can be imagined, the axial velocities near the pipe wall will increase more than those far away from the wall (and thus close to the centerline). Similarly, outflow decreases axial velocities near the wall more significantly than those away from the wall. As a result, the velocity gradient on and near the pipe wall increases due to the inflow while it decreases due to outflow. Correspondingly, the wall friction increases for inflow case but decreases for outflow.

- Turbulent Flow: with the presence of mass transfer through perforations, the time average velocity profile for turbulent pipe flow is altered due to the interaction between the axial flow and the perforation flow. Inflow lifts and expands the turbulent boundary layer and thus increases the axial velocity beyond the layer while decreases the velocity within the layer to follow the mass conservation law. As a consequence the axial velocity gradient near the pipe wall decreases and so does the wall friction shear stress. On the contrary, outflow lowers and reduces the boundary layer and thus decreases the average velocity outside the layer but increases the velocity inside the layer, which results in an increase of the axial velocity gradient near the pipe wall and hence the wall friction shear stress. The analysis is consistent with the numerical observations of Kinney \& Sparrow [3] for pipe flow with suction through pipe wall.

\subsection{Single Phase Wellbore Flow Model}

Consider fluid flow in a wellbore as shown in Figure 1.1 and assume single phase flow of an incompressible Newtonian fluid under isothermal conditions with no heat transfer to and from the fluid. Furthermore, assume that no mechanical work is done on or by the fluid during its passage through the pipe. With these assumptions, the momentum balance equation takes the form (see Ouyang et al. [2] for details) 


$$
\begin{aligned}
{\left[(p A)_{2}-(p A)_{1}\right]=} & {\left[\frac{1}{\alpha_{1} g_{c}} \rho A_{1} U_{1}^{2}-\frac{1}{\alpha_{2} g_{c}} \rho A_{2} U_{2}^{2}\right] } \\
& +\frac{n \triangle x}{\alpha_{I} g_{c}} \rho A_{I} U_{r} U_{x}-\tau_{w} S \triangle x \\
& -\rho \bar{A} \triangle x \frac{g}{g_{c}} \sin \theta
\end{aligned}
$$

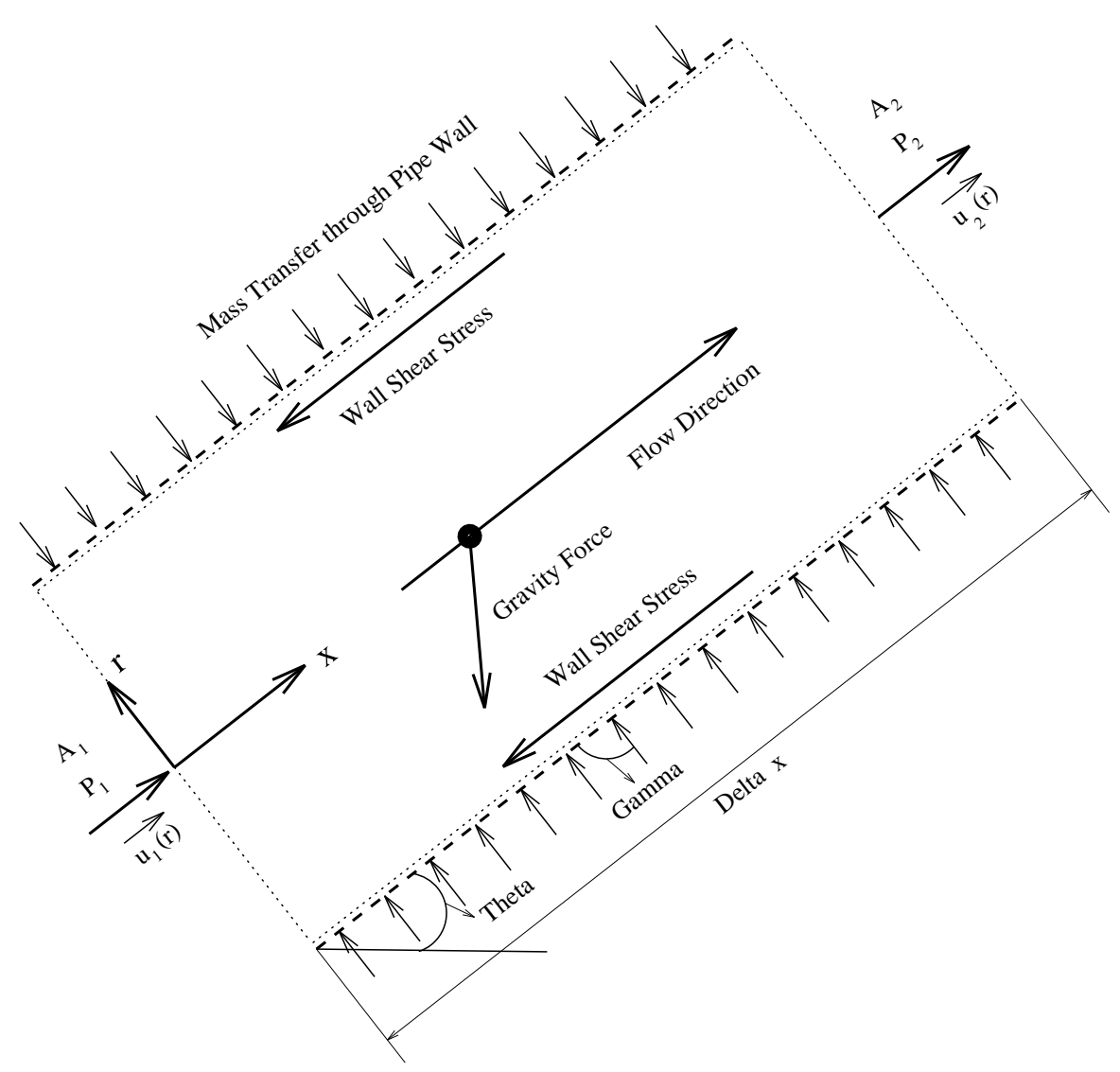

Figure 1.1: Mass and Momentum Transfer along a Wellbore with Mass Transfer through Perforations

which can be rearranged in combination with mass balance equation to give the following pressure gradient equation

$$
\begin{aligned}
\frac{d p}{d x}= & -n \frac{A_{I}}{A} \frac{2 \rho}{g_{c} \alpha} U U_{I}-\tau_{w} \frac{S}{A} \\
& +n \frac{A_{I}}{A} \frac{\rho}{2 g_{c} \alpha_{I}} U_{I}^{2} \sin 2 \gamma-\rho \frac{g}{g_{c}} \sin \theta
\end{aligned}
$$

where $\gamma$ is the injection angle (Figure 1.1), $A_{I}$ the cross-section area of each perforation.

Eq. 1.2 indicates that the overall pressure gradient consists of four different components: 
- The pressure gradient due to kinetic energy change (accelerational effects). This term should be zero for incompressible fluid flow in pipes of constant ID and without inflow or outflow. Obviously, it will not be zero for wellbore flow with influx.

- The frictional pressure gradient. This part of pressure gradient depends on both axial and perforation flows.

- The pressure gradient due to the inflow direction, and it is termed as inflow-directional pressure drop in this paper. It may help or hinder the axial flow depending on the inflow direction.

- The gravitational pressure gradient. It is reasonable to assume that the gravitational pressure gradient is trivial and thus negligible for horizontal wellbore flow.

In order to quantitatively describe the relative importance of different pressure gradient components, the following three dimensionless numbers are introduced:

$R_{a f}$ is defined as the ratio of the accelerational pressure gradient and the frictional pressure gradient;

$R_{g f}$ is defined as the ratio of the gravitational pressure gradient and the frictional pressure gradient;

$R_{d a}$ is defined as the ratio of the directional pressure gradient and the accelerational pressure gradient.

Therefore, the total pressure gradient can be expressed as:

$$
\frac{d p}{d x}=-\frac{4 \tau_{w}}{D}\left[1+R_{a f}\left(1-R_{d a}\right)+R_{g f}\right]
$$

Ouyang et al. $[1,2]$ found that the closer we are to the horizontal well toe $(x=0)$, the smaller the local wellbore production rate $q_{w}$, thus the larger the $R_{a f}$ and the more important the accelerational pressure gradient. In contrast, near the heel of the horizontal well $(x=L)$, the local production rate $q_{w}$ becomes large and close to the total well production rate, while the specific influx (inflow rate per wellbore length) $q_{e}$ does not change significantly, so $R_{a f}$ is small, and the accelerational pressure gradient is small and may be negligible.

For the uniform inflow case, we have shown in Ref. [2] that:

- In the laminar flow regime $\left(x \leq L_{t}\right), R_{a f}$ is only dependent on fluid properties, inflow rate and pipe ID, but it is independent of location $x$ and pipe roughness $\epsilon$. The larger the $q_{e}$, the larger the $R_{a f}$, but the shorter the laminar flow length $L_{t}$.

- In the turbulent flow regime $\left(x>L_{t}\right)$, the Fanning friction factor $f$ is dependent on the local Reynolds number, the wall Reynolds number and the relative pipe roughness, as a consequence, the $R_{a f}$ depends on location $x$, pipe geometry (pipe ID and pipe roughness), fluid properties, and inflow rates. 
It is anticipated that turbulent flow occurs along almost the whole wellbore section for most practical situations. Furthermore, the momentum correction factor $\alpha$ does not change much for different velocity profiles under turbulent flow conditions (Ouyang \& Aziz, [4]), therefore, $\alpha$ can be assumed constant. As a result, Eq. 1.2 can be rewritten as

$$
\tau_{w}=\frac{D}{4}\left[\frac{d p}{d x}-n \frac{A_{I}}{A} \frac{2 \rho}{g_{c} \alpha} U U_{I}+n \frac{A_{I}}{A} \frac{\rho}{2 g_{c} \alpha_{I}} U_{I}^{2} \sin 2 \gamma-\rho \frac{g}{g_{c}} \sin \theta\right]
$$

Based on Eq. 1.4, the wall friction shear stress and hence the wall friction factor can be calculated. The wall friction factor is expected to depend on the local axial Reynolds number, the injection Reynolds number, the effective relative pipe roughness and maybe the inflow/axial flow rate ratios.

\subsection{New Wall Friction Factor Correlations}

The mass transfer through the pipe wall affects wall friction shear. The influence of either inflow or outflow depends on the flow regime present in the wellbore. The inflow (injection) increases the wall friction for laminar flow while decreases it for turbulent flow. On the contrary, outflow (suction) decreases the wall friction for laminar flow while increases the friction for turbulent flow. In other words, the wall friction is altered compared to the pipe flow where no inflow or outflow is present. Therefore, the friction factor correlations for pipe flow without inflow or outflow can not be used for wellbore flow with both axial flow in the pipe and inflow or outflow through perforations.

\subsubsection{Laminar Flow in a Porous Pipe}

Based on the reduced ordinary differential equation for the dimensionless streamfunction and the numerical procedure provided in Kinney [5], the ratios between the local friction factor and the no-wall-flow friction factor corresponding to different wall Reynolds numbers $R_{e w}$ were calculated. These data have been used to develop the following friction factor correlations by means of a fast and effective nonlinear regression procedure (Ouyang et al., $[1,2])$.

- For inflow (injection) case

$$
f=\frac{16.0}{R_{e}}\left(1+0.04304 R_{e w}^{0.6142}\right)
$$

- For outflow (suction or extraction) case

$$
f=\frac{16.0}{R_{e}}\left[1-0.0625 \frac{\left(-R_{e w}\right)^{1.3056}}{\left(R_{e w}+4.626\right)^{-0.2724}}\right]
$$

\subsubsection{Turbulent Flow in a Porous Pipe}

A new correlation for the local wall friction factor for turbulent flow has also been developed in the present paper by using Olson \& Eckert's experimental data [6] for turbulent 
air flow in a porous pipe with uniform air injection through the pipe wall. The new correlation is:

$$
f=f_{0}\left[1-29.03\left(\frac{R_{e w}}{R_{e}}\right)^{0.8003}\right]
$$

It is found that the new correlation can provide satisfactory prediction of wall friction factor for porous pipe flow with injection through the pipe wall. The no-wall-flow friction factor $f_{0}$ can be determined either from the Colebrook-White $[7,8]$ equation or from one of its explicit approximations (Ouyang \& Aziz, [4]).

For turbulent pipe flow with outflow (suction) through porous pipe wall, the local friction factor can be evaluated by the Kinney-Sparrow-Wallis's correlation [3]:

$$
f=f_{0}\left[1-17.5 \frac{R_{e w}}{R_{e}^{0.75}}\right]
$$

\subsubsection{Wellbore Flow}

Both correlations (Eqs. 1.7 and 1.8) are based on experimental data for porous pipe flow. It is unclear whether they can also be used for normal wellbore flow conditions. We have applied Eq. 1.7 to analyse the single phase wellbore flow data from the 1995 stanford horizontal wellbore experiments and found that it overpredicts the wall friction factor. Therefore, another new correlation for the local friction factor has been developed for wellbore turbulent flow based on the 1995 Stanford horizontal wellbore experiment data:

$$
f=f_{0}\left[1-0.0153 R_{e w}^{0.3978}\right]
$$

\subsection{Results and Discussions}

\subsubsection{Flow Regimes Present in a Wellbore}

The changes of accelerational to frictional pressure gradient ratio, $R_{a f}$, with distance along the wellbore for uniform-influx horizontal wells with different wellbore lengths, production rates, and oil viscosities indicate that three regions exist along the wellbore, the laminar flow region, the partially-developed turbulent flow region, and the fully-developed turbulent flow region. The dimensionless number $R_{a f}$ is a constant in the laminar flow region that depends on fluid properties (viscosity and density), production rate and pipe geometry (pipe ID and pipe roughness). If all other parameters are fixed, then the shorter the horizontal well, or the higher the production rate, or the more viscous the oil, the larger the value of $R_{a f}$, but the shorter the length of the laminar flow region. For the fully-developed turbulent flow region, all the $R_{a f}$ curves for different production rates, different fluid properties, and different wellbore lengths, merge into one, which only changes with wellbore location. Between the laminar and the fully-developed turbulent flow region lies the partially-developed turbulent flow region where $R_{a f}$ varies with wellbore location, fluid properties, production rate and pipe geometry. 


\subsubsection{Partially-Perforated Horizontal Well Example}

A partially-perforated horizontal well refers to a well where only part of the wellbore is perforated to allow inflow. Also, it is assumed that the wellbore flow rate before the perforated zone, $Q_{0}$, may or may not be zero.

Figures 1.2 and 1.3 show pressure gradients and cumulative pressure drops from the start of the perforations in a partially-perforated horizontal well (well \#1, see Table 1.1). Also shown in Figure 1.2 is the frictional pressure gradient determined from the no-wall-flow friction factor $f_{0}$. It is found that there is a nontrivial difference between the actual frictional pressure gradient and the frictional pressure gradient obtained from the no-wall-flow friction factor. The accelerational pressure gradient is comparable to the frictional component, and the dimensionless number $R_{a f}$ changes from 1.27 at the starting point of the well perforation to 0.71 at the end of the perforation. The cumulative accelerational pressure drop from the starting point of the perforations is more or less the same as the cumulative frictional pressure drop (Figure 1.3).

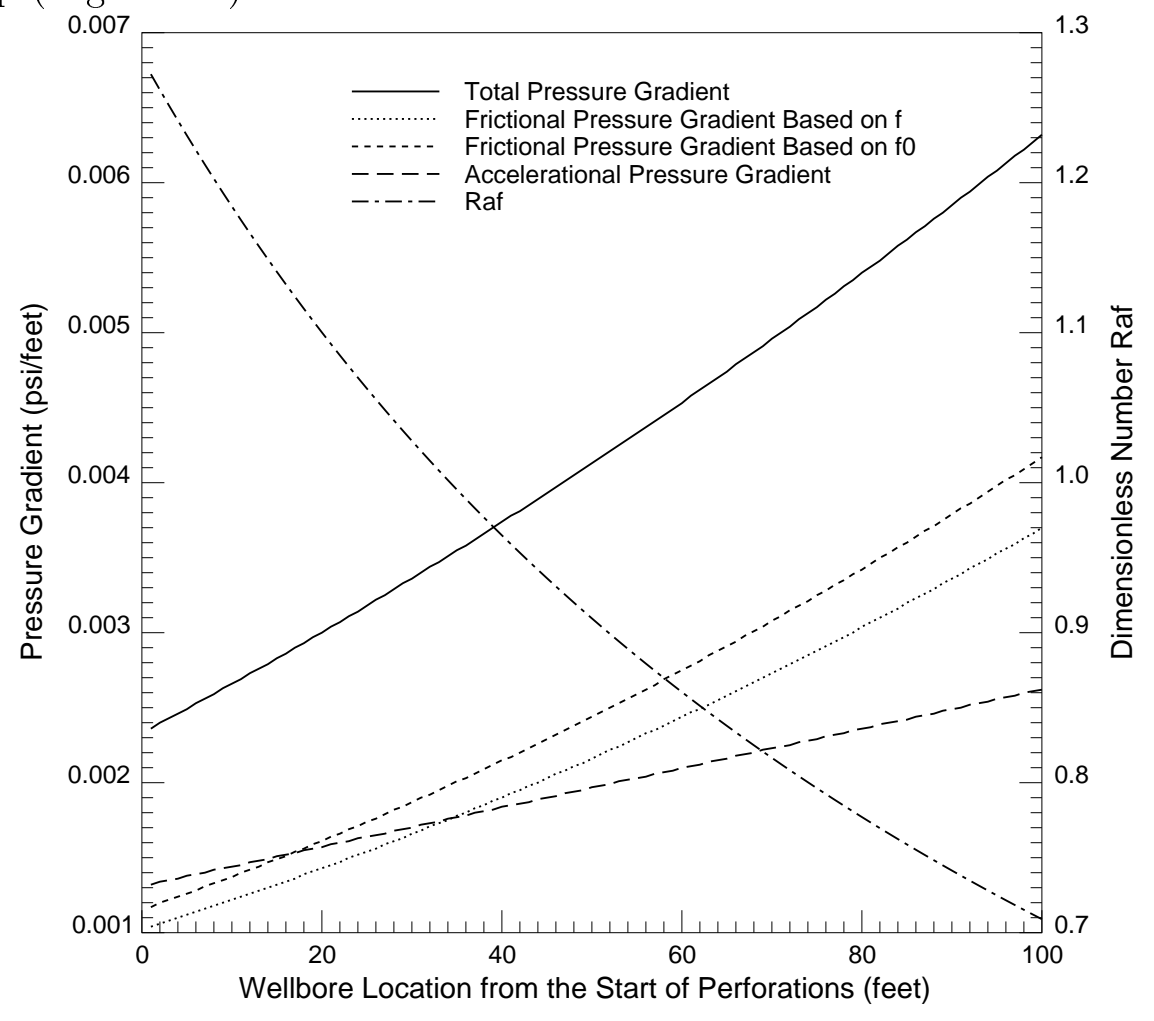

Figure 1.2: Pressure Gradients Change with Wellbore Location (well \#1)

\subsubsection{Fully-Perforated Horizontal Well Example}

In contrast to partially-perforated horizontal wells, fully-perforated horizontal wells have perforations over the entire wellbore length.

Figure 1.4 shows the change in pressure gradients with wellbore location for horizontal well \#2 (see Table 1.1). In this case, the dimensionless pressure gradient ratio $R_{a f}$ ranges from 0.8 to 0.05 , and the cumulative accelerational pressure drop is only about $6 \%$ of the total pressure drop along the whole wellbore, hence the accelerational pressure gradient is 


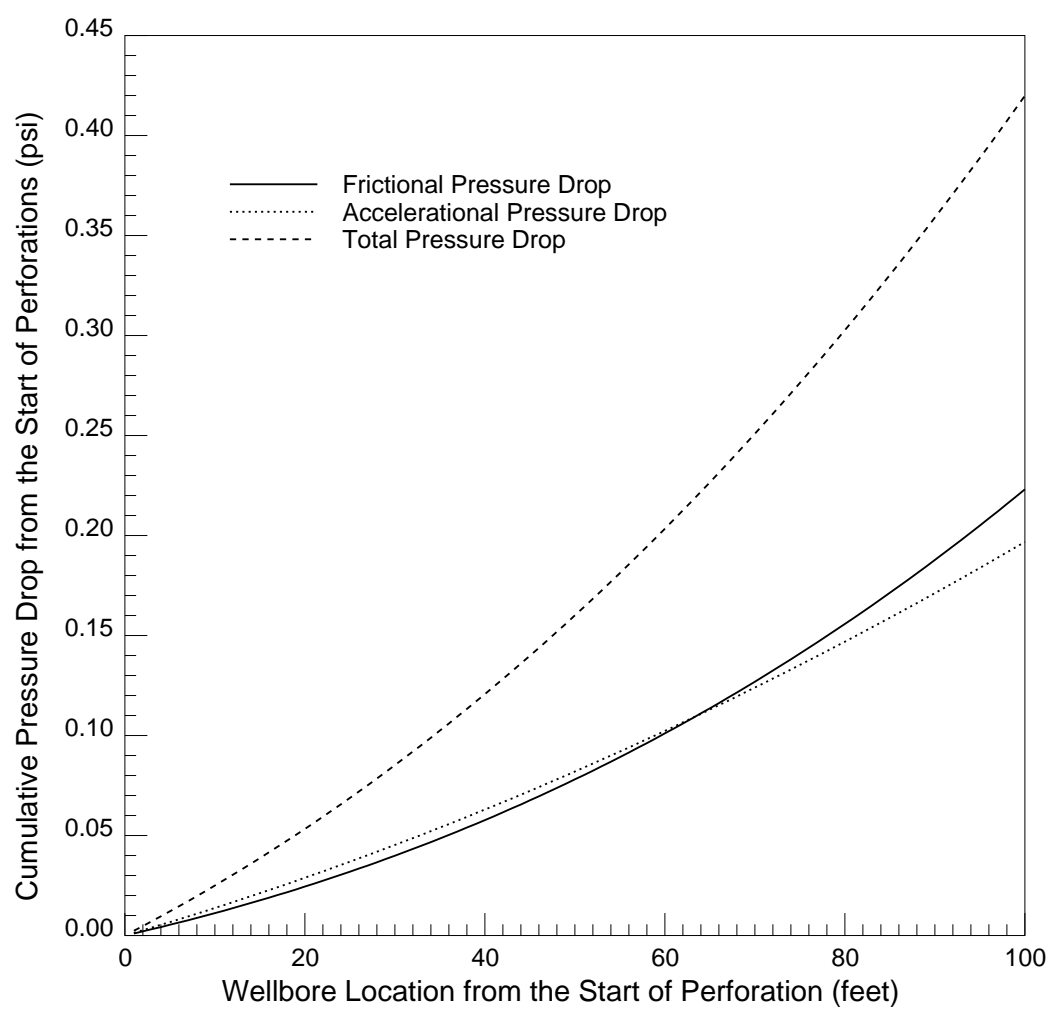

Figure 1.3: Change in Cumulative Pressure Drops with Wellbore Location (well \#1)

quite small compared to the frictional component for this horizontal well and only minor errors will result if the accelerational pressure drop is neglected.

\subsubsection{Coupling the New Wellbore Model with a Reservoir Inflow Model}

It is assumed in the above discussion that the specific inflow rate (i. e. the inflow rate per unit wellbore length) is the same everywhere along the whole wellbore. This is not necessarily true in a real horizontal well, since the wellbore pressure near the well toe is higher than that at the well heel due to wellbore pressure drop, the pressure drawdown near the well toe is less than that near the well heel, therefore, the specific inflow rate near the well toe should be smaller than that near the well heel. On the one hand, wellbore pressure or pressure drop is needed to determine the specific inflow rate distribution along the wellbore. On the other hand, the specific inflow rate distribution is an indispensable parameter to calculate wellbore pressure or pressure drop. Hence, a model which incorporates both the wellbore fluid flow and the fluid flow from reservoir into wellbore is required for determining specific inflow rate distribution, wellbore pressure drop, and overall well production rate.

Since the reservoir inflow model is not the main issue of this paper, a simple reservoir model as used by Dikken [9] and Novy [10] is applied to describe the reservoir fluid flow:

$$
q_{e}(x)=J_{s}\left[p_{e}-p_{w}(x)\right]
$$

As discussed above, the total pressure gradient for horizontal wells consists of three components, frictional, accelerational and inflow-directional. For the horizontal well \#3 (Table 1.1), the frictional component contributes the largest part of the total pressure gradient, 


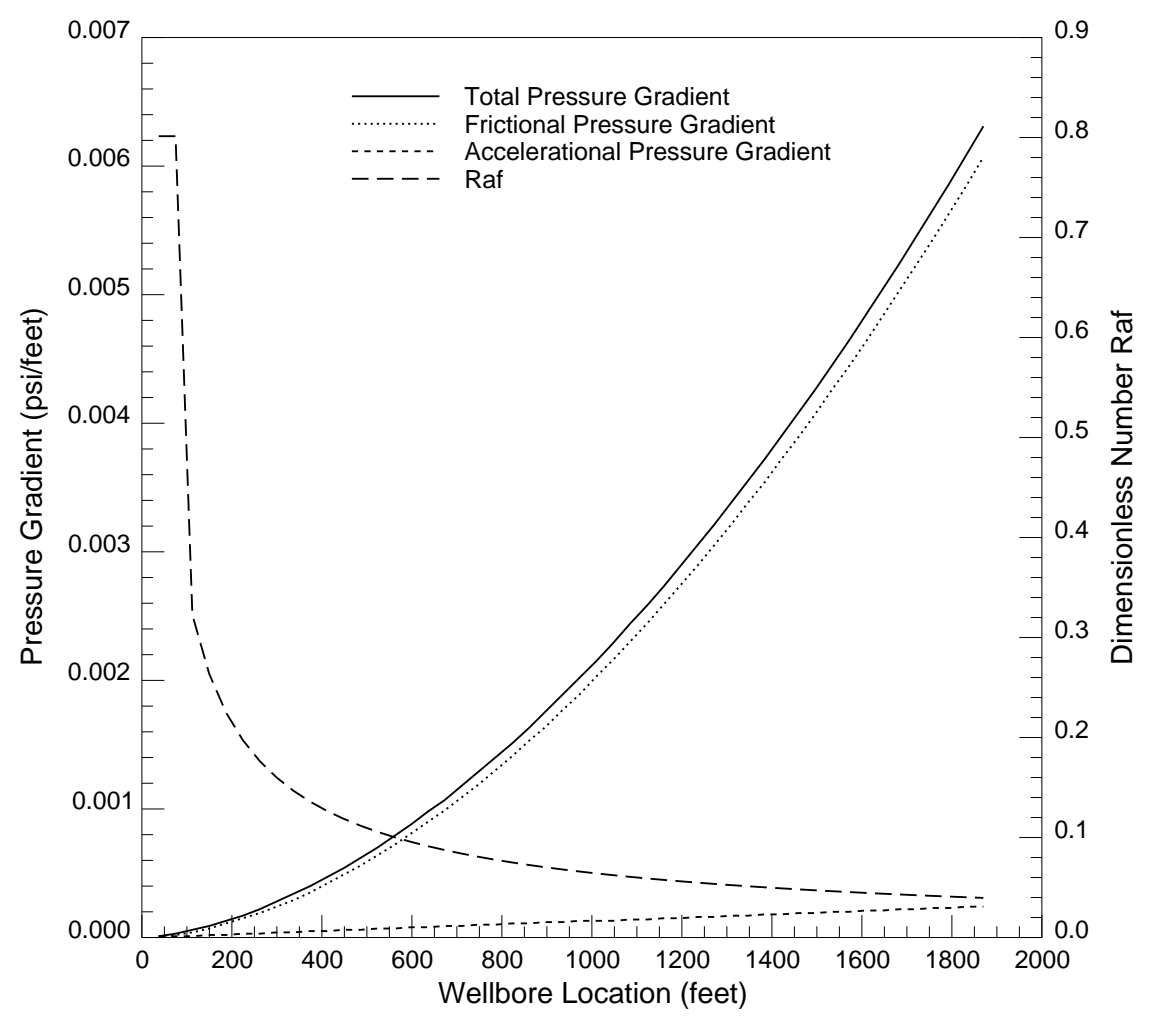

Figure 1.4: Pressure Gradients Change with Wellbore Location (well \#2)

the accelerational component contributes in the range of $20 \%$ to $80 \%$ depending on the wellbore location $\left(R_{a f}\right.$ changes from about 7.0 to 0.25$)$, while the inflow-directional component contributes very little and can be neglected (Figure 1.5). Correspondingly the cumulative pressure drop along the wellbore is primarily caused by wall friction (which is also affected by inflow), while acceleration or kinetic energy changes only contribute about $5 \%$ to $10 \%$ to the overall pressure drop (Figure 1.6).

Specific inflow rate is not constant along the wellbore due to wellbore pressure drop (Figure 1.7). Significant differences occur between the real specific inflow rate predicted by considering pressure drop along the wellbore and the fictitious specific inflow rate where no pressure drop along the wellbore is assumed. For more accurate prediction of the specific inflow rate distribution, accelerational pressure drop should also be included in the wellbore model, otherwise around $10 \%$ overestimation of the specific inflow rate is likely to occur.

The well production rate is proportional to wellbore length if no pressure drop occurs along wellbore. The statement is invalid for many real horizontal wells. Figure 1.8 shows the production rate change with wellbore length. Well production rate decreases significantly due to pressure drop in the wellbore, and is likely to reach a maximum value at certain wellbore length beyond which no additional production is expected, no matter how long the horizontal well is. The well production rate decreases further if both frictional and accelerational pressure drops are included.

\subsection{Conclusions}

A general single phase wellbore flow model, which incorporates not only frictional, accelerational and gravitational pressure drops, but also the pressure drop caused by inflow, is 


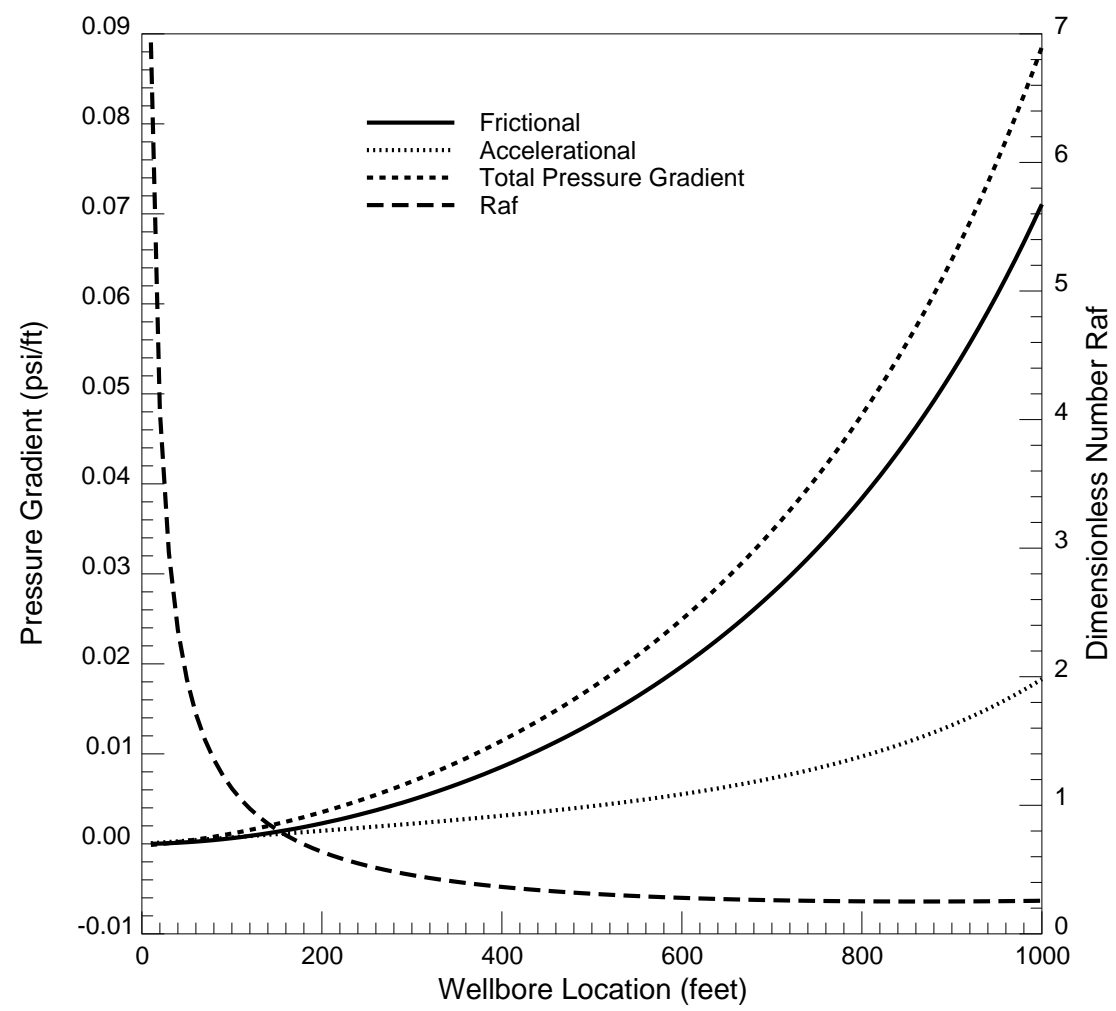

Figure 1.5: Pressure Gradients Change with Wellbore Location (well \#3)

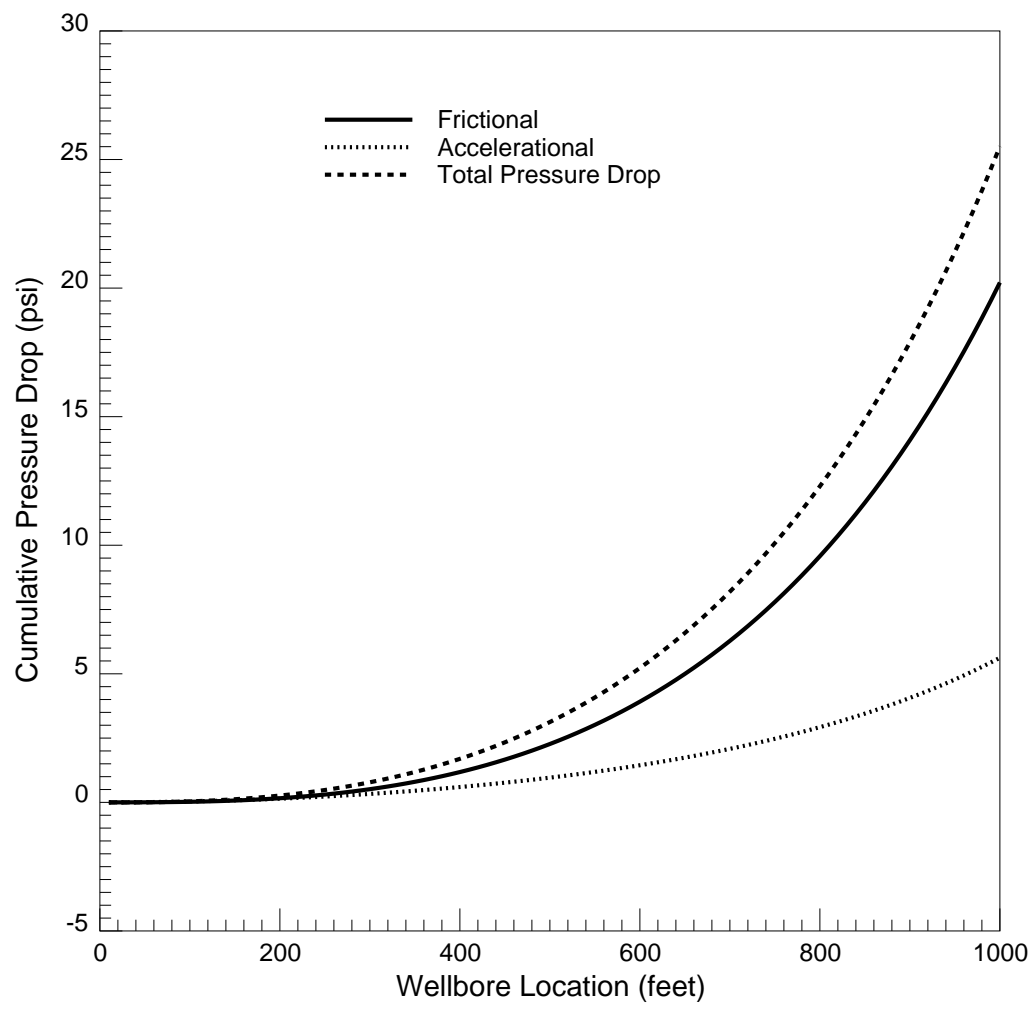

Figure 1.6: Change in Cumulative Pressure Drops with Wellbore Location (well \#3) 


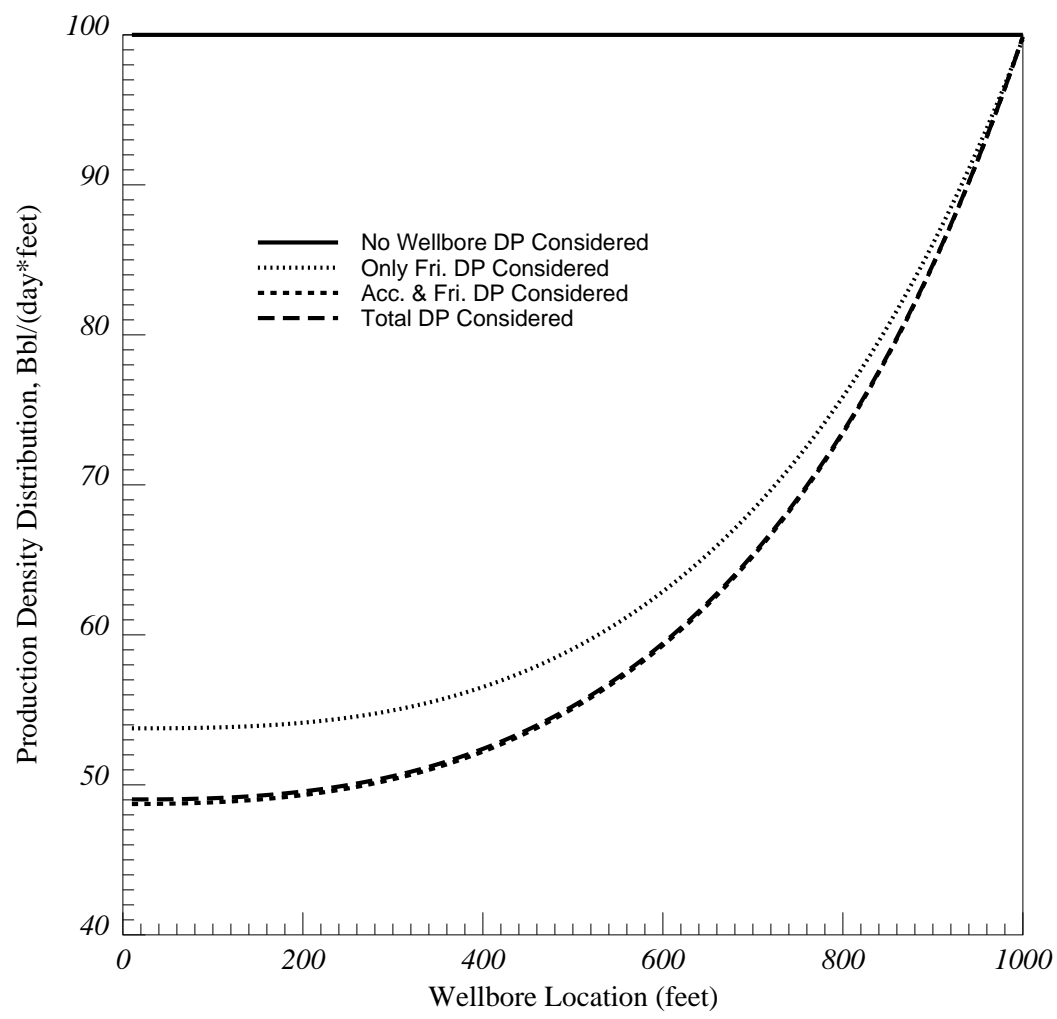

Figure 1.7: Specific Inflow Rate Distribution (well \#3)

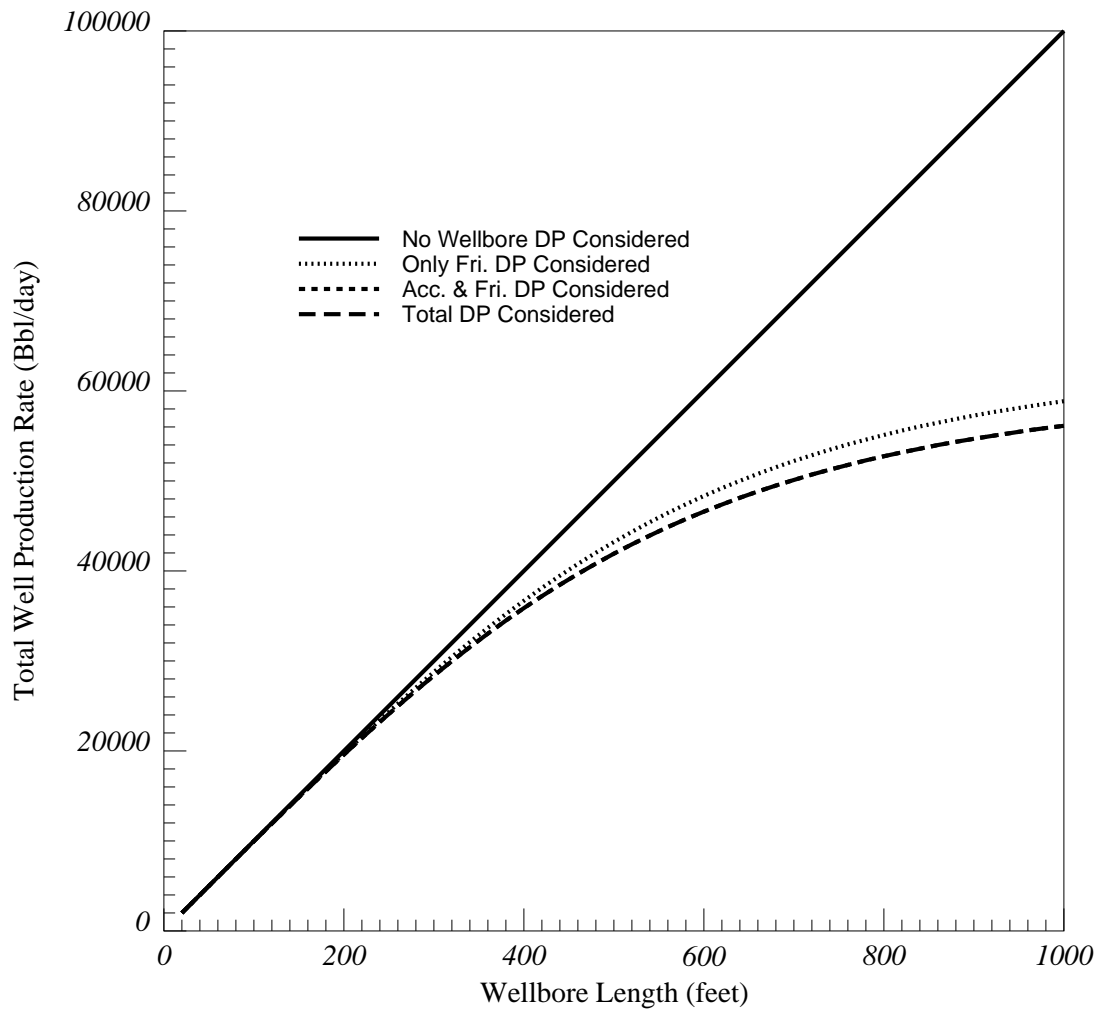

Figure 1.8: Overall Well Production Rate Change with Wellbore Length (well \#3) 
Table 1.1: Horizontal Well Parameters

\begin{tabular}{|c|c|c|c|}
\hline Parameter & Well \#1 & Well \#2 & Well \#3 \\
\hline Wellbore Length (feet) & 110 & 1870 & 1000 \\
\hline Perforated Interval (feet) & 100 & 1870 & 1000 \\
\hline Pipe ID (inch) & 6.18 & 4.5 & 6 \\
\hline Relative Pipe Roughness & $2.0 \times 10^{-4}$ & $1.0 \times 10^{-4}$ & $2.0 \times 10^{-4}$ \\
\hline Perforation ID (inch) & 0.18 & 0.18 & 0.18 \\
\hline Perforation Density $\left(\frac{h o l e}{\text { feet }}\right)$ & 8 & 10 & 10 \\
\hline Inflow Direction ( deg) & 90 & 45 & 45 \\
\hline Fluid Density $\left(\frac{b m}{f t^{3}}\right)$ & 62.4 & 52.44 & 62.0 \\
\hline Fluid Viscosity $(c p)$ & 0.878 & 2.5 & 1.0 \\
\hline Wellbore Rate before Completion Point $\left(\frac{B b l}{d a y}\right)$ & 7000 & N/A & N/A \\
\hline Inflow Rate $\left(\frac{B b}{d a y}\right)$ & 7000 & 7500 & N/A \\
\hline Specific Inflow Rate $\left(\frac{B b l}{\text { day } \times \text { feet }}\right)$ & $\mathrm{N} / \mathrm{A}$ & $\mathrm{N} / \mathrm{A}$ & 2.0 \\
\hline
\end{tabular}

presented. The new wellbore model is readily applicable to any wellbore perforation patterns and well completions, and can be easily incorporated in reservoir simulators or analytical reservoir inflow models.

Inflow and outflow affect wall friction shear. The influence of either inflow or outflow depends on the flow regime present in the wellbore. Inflow (production wells) increases the wall friction for laminar flow while decreases the friction for turbulent flow. The opposite is true for outflow (injection wells).

New wall friction factor correlations for pipe flow with influx have been developed based on published data and the Stanford horizontal wellbore experiments. They can be applied for wellbore flow to determine wall friction shear and thus frictional pressure drop for either inflow (production well) or outflow (injection well) and for either laminar flow or turbulent flow.

It is shown that accelerational pressure drop may or may not be important compared to the frictional component depending on the specific pipe geometry, fluid properties and flow conditions. 


\section{Nomenclature}

A pipe cross-section area

$D$ pipe internal diameter (ID)

$D_{I}$ perforation internal diameter

$f \quad$ Fanning friction factor

$f_{0} \quad$ no-wall-flow Fanning friction factor

$f_{T} \quad$ total or apparent Fanning friction factor

$g$ acceleration due to gravity

$g_{c} \quad$ conversion factor

$L \quad$ wellbore length

$L_{t} \quad$ wellbore length for laminar flow region

$n$ perforation density

$p$ pressure

$q_{e} \quad$ specific inflow rate

$q_{w} \quad$ local wellbore flow rate

$R_{a f}$ ratio between accelerational and frictional pressure gradient

$R_{g f}$ ratio between gravitational and frictional pressure gradient

$R_{d a}$ ratio between inflow-directional and accelerational pressure gradient

$R_{e} \quad$ Reynolds number

$R_{e w}$ wall Reynolds number, positive for injection and negative for suction

$S \quad$ wellbore perimeter

$U$ average axial velocity

$U_{n} \quad$ average velocity for no inflow or outflow case

$U_{w}$ average velocity for inflow or outflow case

$U_{\infty}$ free stream velocity

$V$ volume

$V_{w} \quad$ wall inflow or outflow velocity

$x$ axial coordinate

\section{Greek Letters}

$\alpha \quad$ momentum correction factor

$\gamma \quad$ inflow direction angle

$\delta \quad$ boundary layer thickness

$\theta \quad$ wellbore inclination angle

$\mu \quad$ fluid viscosity

$\nu \quad$ kinetic fluid viscosity

$\rho \quad$ fluid density

$\tau_{w} \quad$ wall friction shear stress

\section{Subscripts}

acc accelerational

ele elevational or gravitational 
fri frictional

\section{References}

[1] L-B Ouyang, S. Arbabi, and K. Aziz. "General Wellbore Flow Model for Horizontal, Vertical and Slanted Well Completions". In The 71st Annual SPE Technical Conference \& Exhibition, Denver, Colorado, 1996. Paper SPE 36608.

[2] L-B Ouyang, S. Arbabi, and K. Aziz. "General Single Phase Wellbore Flow Model". Technical Report for U.S. Department of Energy, Stanford University, CA, 1996.

[3] R. B. Kinney and E. M. Sparrow. "Turbulent Flow, Heat Transfer and Mass Transfer in a Tube with Surface Suction". J. Heat Transfer, 92:117-125, 1970.

[4] L-B Ouyang and K. Aziz. "Steady-State Gas Flow in Pipes". J of Petroleum Science and Engineering, 14:137-158, 1996.

[5] R.B. Kinney. "Fully Developed Frictional and Heat-transfer Characteristics of Laminar Flow in Porous Tubes". Int J Heat Mass Transfer, 11:1393-1400, 1968.

[6] R. M. Olson and E. R. G. Eckert. "Experimental Studies of Turbulent Flow in a Porous Circular Tube with Uniform Fluid Injection through the Tube Wall". J of Applied Mechanics, 33(1):7-17, 1996.

[7] C. F. Colebrook and C. M. White. "Experiments with Fluid Friction in Roughened Pipes". Proc. Roy Soc. (London), 161A:367-381, 1937.

[8] C. F. Colebrook. "Turbulent Flow in Pipes, with Particular Reference to the Transition Region between the Smooth and Rough Pipe Laws". J. Instn. Civil Engrs. (London), 12:133-156, 1939.

[9] B. J. Dikken. "Pressure Drop in Horizontal Wells and its Effect on Production Performance". J of Petroleum Technology, 42(11):1426-1433, 1990.

[10] R. A. Novy. "Pressure Drops in Horizontal Wells: When Can They Be Ignored?", SPE Reservoir Engineering, 10(1):29-35,1995. 


\title{
2. Development and Testing of a New Mechanistic Model for Multiphase Flow in Pipes
}

\author{
by Nicholas Petalas and Khalid Aziz
}

\begin{abstract}
A revised stratified flow model is presented including new correlations for the friction factors at the gas/liquid interface and at the wall/liquid interface. The mechanistic model is tested after incorporating the new stratified flow model and the test results are presented and discussed.
\end{abstract}

\subsection{Introduction}

The development of the mechanistic model started with a preliminary version that was developed based on the best correlations found in the currently available literature. The model's performance was then evaluated through the use of graphical techniques ${ }^{1}$ over the complete range of pipe geometries, fluid flow rates and properties.

To aid in the testing of the model with actual data, the Stanford Multiphase Flow Database $^{4}$ (SMFD), was developed. The model's performance was then tested through comparisons with experimental data from the SMFD and it was also compared to existing mechanistic models and empirical correlations.

\subsection{Recent developments}

Improvements to the mechanistic model are being implemented by independently analyzing each flow regime using the SMFD and developing, where required, new empirical correlations to replace those previously selected. The first flow pattern to be investigated was the annular-mist regime and new correlations were developed for the gas/liquid interfacial friction factor and the liquid fraction entrained. The resulting model, including the comparisons with existing models, was discussed in a report ${ }^{6}$ issued in April 1996 and presented in a paper ${ }^{5}$ at the summer meeting of the ASME in July 1996.

In the work that has since ensued, the stratified flow regime was examined and the model was revised as described below. In addition, the model was tested using 79 actual field measurements provided by BP and was reportedly accurate to within $6 \%$. The field data, however, have not as of yet been made available to us.

A version of the mechanistic model of $\mathrm{Xiao}^{7}$ et. al. was obtained through private communication with J.J. Xiao. This model differs significantly from the published version which, in previous comparisons with the present model, was shown to be quite poor. The new 
version has been implemented and although the results are significantly improved, the Stanford model is still superior to all other models tested.

\subsection{Revised Stratified Flow Model}

The geometrical aspects of stratified flow are shown in Figure 2.1.

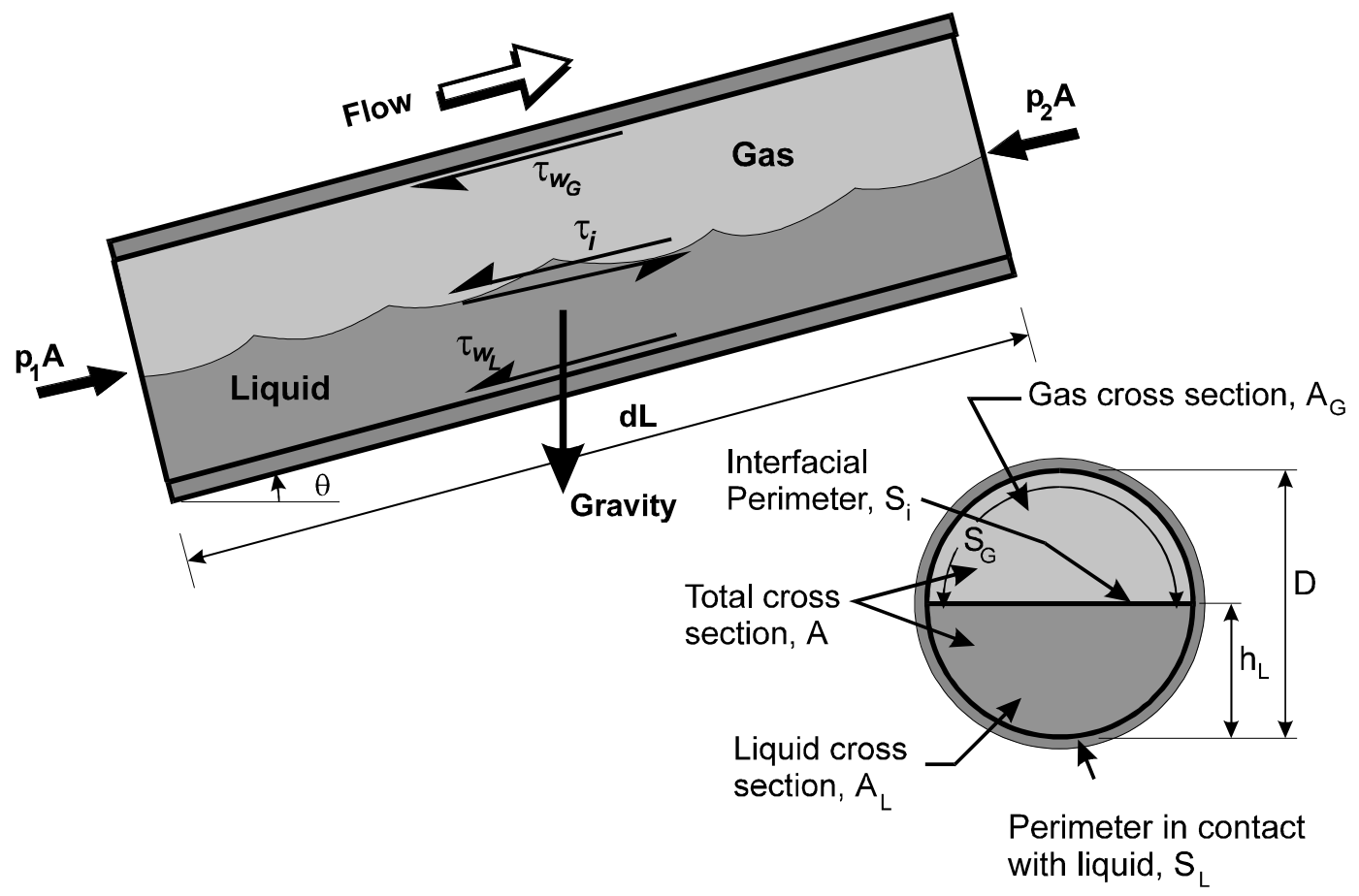

FIGURE 2.1 - MOMENTUM BALANCE WITH TWO SEGREGATED FLUIDS

A momentum balance can be written on the liquid and gas phases contained in a control volume. Thus, for the liquid phase:

$$
-\frac{p_{2} A_{L}}{\Delta L}+\frac{p_{1} A_{L}}{\Delta L}-\tau_{w L} S_{L}+\tau_{i} S_{i}-\rho_{L} A_{L} \frac{g}{g_{c}} \sin \theta=0
$$

Similarly, for the gas phase:

$$
-\frac{p_{2} A_{G}}{\Delta L}+\frac{p_{1} A_{G}}{\Delta L}-\tau_{w G} S_{G}-\tau_{i} S_{i}-\rho_{G} A_{G} \frac{g}{g_{c}} \sin \theta=0
$$

Note that the interfacial shear stress, $\tau_{i}$, is positive if the gas phase is flowing faster than the liquid phase, i.e. $V_{\mathrm{G}}>V_{\mathrm{L}}$. After taking the limit as $\Delta \mathrm{L} \rightarrow 0$ the two momentum balance equations may be expressed as:

$$
-A_{L}\left(\frac{d p}{d L}\right)-\tau_{w L} S_{L}+\tau_{i} S_{i}-\rho_{L} A_{L} \frac{g}{g_{c}} \sin \theta=0
$$

and 


$$
-A_{G}\left(\frac{d p}{d L}\right)-\tau_{w G} S_{G}-\tau_{i} S_{i}-\rho_{G} A_{G} \frac{g}{g_{c}} \sin \theta=0
$$

The shear stress terms can be expressed in terms of friction factors:

$$
\tau_{w L}=\frac{f_{L} \rho_{L} V_{L}^{2}}{2 g_{c}}, \tau_{w G}=\frac{f_{G} \rho_{G} V_{G}^{2}}{2 g_{c}} \text {, and } \tau_{i}=\frac{f_{i} \rho_{G} V_{i}\left|V_{i}\right|}{2 g_{c}}
$$

The average interface velocity, $V_{\mathrm{i}}$, is assumed to be given by the difference of the average phase velocities, i.e.

$$
V_{i}=\left(V_{G}-V_{L}\right)
$$

The friction factor for the gas phase is evaluated from the single phase relationship by using the Colebrook ${ }^{3}$ correlation with the following definition of Reynolds number and hydraulic diameter:

$$
\operatorname{Re}_{G}=\frac{D_{G} \rho_{G} V_{G}}{\mu_{G}}, D_{G}=\frac{4 A_{G}}{S_{G}+S_{i}}
$$

In order to determine the friction factors for the gas/liquid interface $\left(f_{\mathrm{i}}\right)$ and for the liquid/wall interface $\left(f_{\mathrm{L}}\right)$ the following empirically derived correlations are used:

$$
\begin{gathered}
f_{i}=0.0104\left(0.004+0.5 \times 10^{-6} \operatorname{Re}_{s L}\right) \operatorname{Fr}_{L}^{1.335}\left\{\frac{\rho_{L} D g}{\rho_{G} V_{G}^{2}}\right\} \\
f_{L}=0.452 f_{S L}^{0.731}
\end{gathered}
$$

The Froude number is defined as $\operatorname{Fr}_{L}=\frac{V_{L}}{\sqrt{g h_{L}}}$.

The evaluation of these relationships is based on data from the Stanford Multiphase Flow Database. A total of 1,026 stratified smooth and stratified wavy experimental measurements were extracted from the database and the following procedure adopted.

By adding Eq. (2.3) to Eq. (2.4) and dividing by the area, the interfacial shear stress term is eliminated and following equation is obtained:

$$
-\frac{d P}{d L}=\frac{\tau_{w L} S_{L}+\tau_{w G} S_{G}}{A}+\left(E_{L} \rho_{L}+E_{G} \rho_{G}\right) \frac{g}{g_{c}} \sin \theta
$$

Using the experimental value of $E_{\mathrm{L}}$, the "experimental" value of the dimensionless liquid height, $\left[h_{\mathrm{L}} / D\right]_{\text {exp }}$, is determined. From this $S_{\mathrm{L}}, S_{\mathrm{G}}$, and $\tau_{\mathrm{wG}}$ are obtained. Now using the experimental value for $(d P / d L)$, the value of $\tau_{\mathrm{wL}}$ can be obtained, and hence $f_{\mathrm{L}}$. By iterating, the value of $f_{\mathrm{i}}$ is determined for which the calculated $h_{\mathrm{L}} / D$ matches $\left[h_{\mathrm{L}} / D\right]_{\text {exp. }}$. This is done for all of the experimental observations and the resulting values of $f_{\mathrm{i}}$ and $f_{\mathrm{L}}$ are correlated to obtain Eq. (2.8) and Eq. (2.9). 


\subsection{Results}

The behavior of the model was examined over a wide range of flow rates and fluid properties using three-dimensional surface plots. This was done over the complete range of upward and downward pipe inclinations and both pressure gradient and volumetric liquid fraction were analyzed. The results indicate much smoother transitions between flow regimes than the previous version of the model.

A comparison with experimental data was also performed. Data were extracted from the Stanford Multiphase Flow Database for which pressure gradient, holdup and flow pattern observations were available. This resulted in a total of 5,951 measurements consisting of variations in fluid properties, pipe diameters, and upward as well as downward inclinations. The model predictions for liquid volume fraction are plotted against the experimental measurements in Figure 2.2. Figure 2.3 shows a similar plot for the pressure gradient calculations.

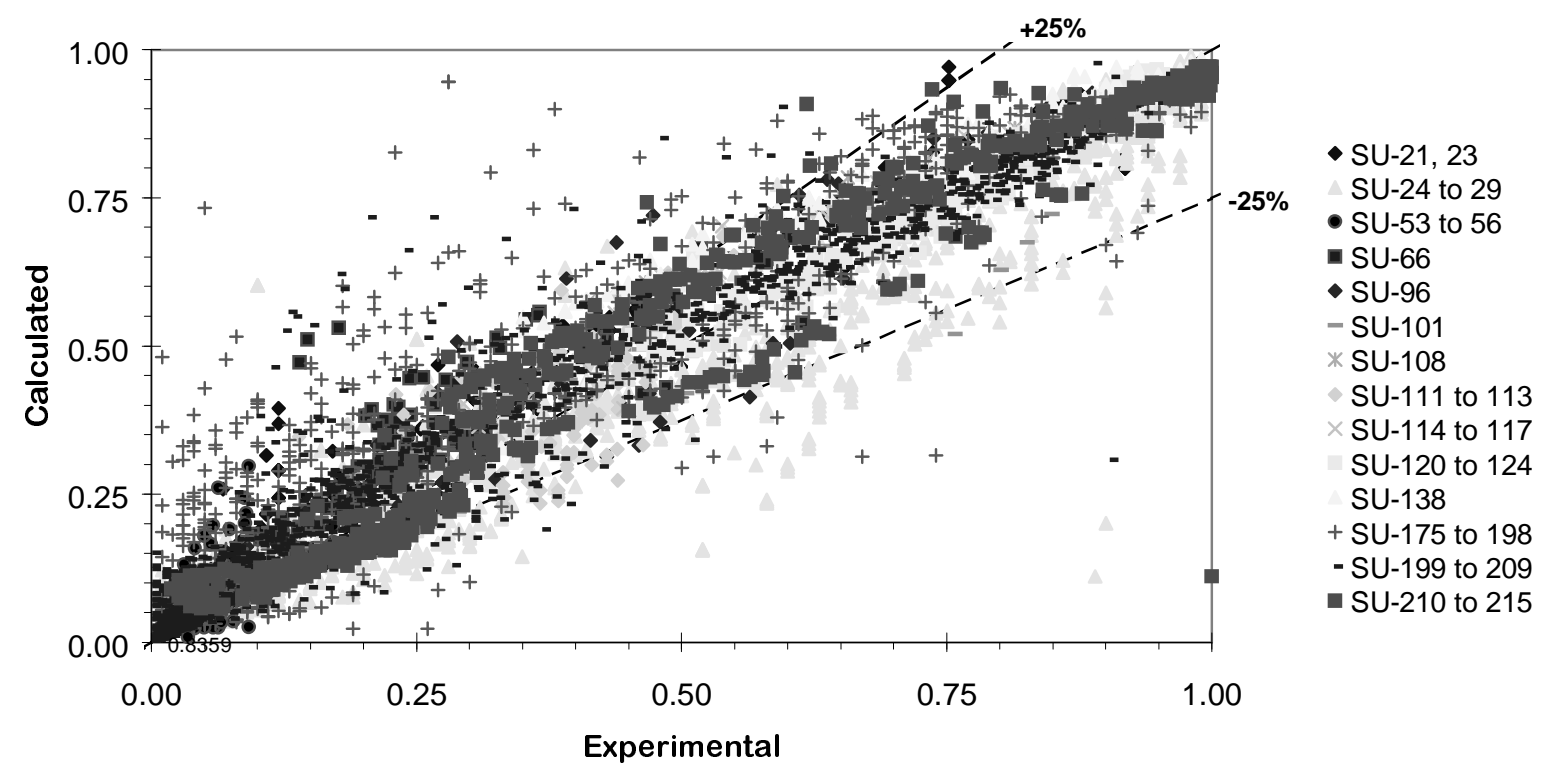

FIGURE 2.2 - COMPARISON OF CALCULATED LIQUID VOLUME FRACTION WITH EXPERIMENTAL DATA 


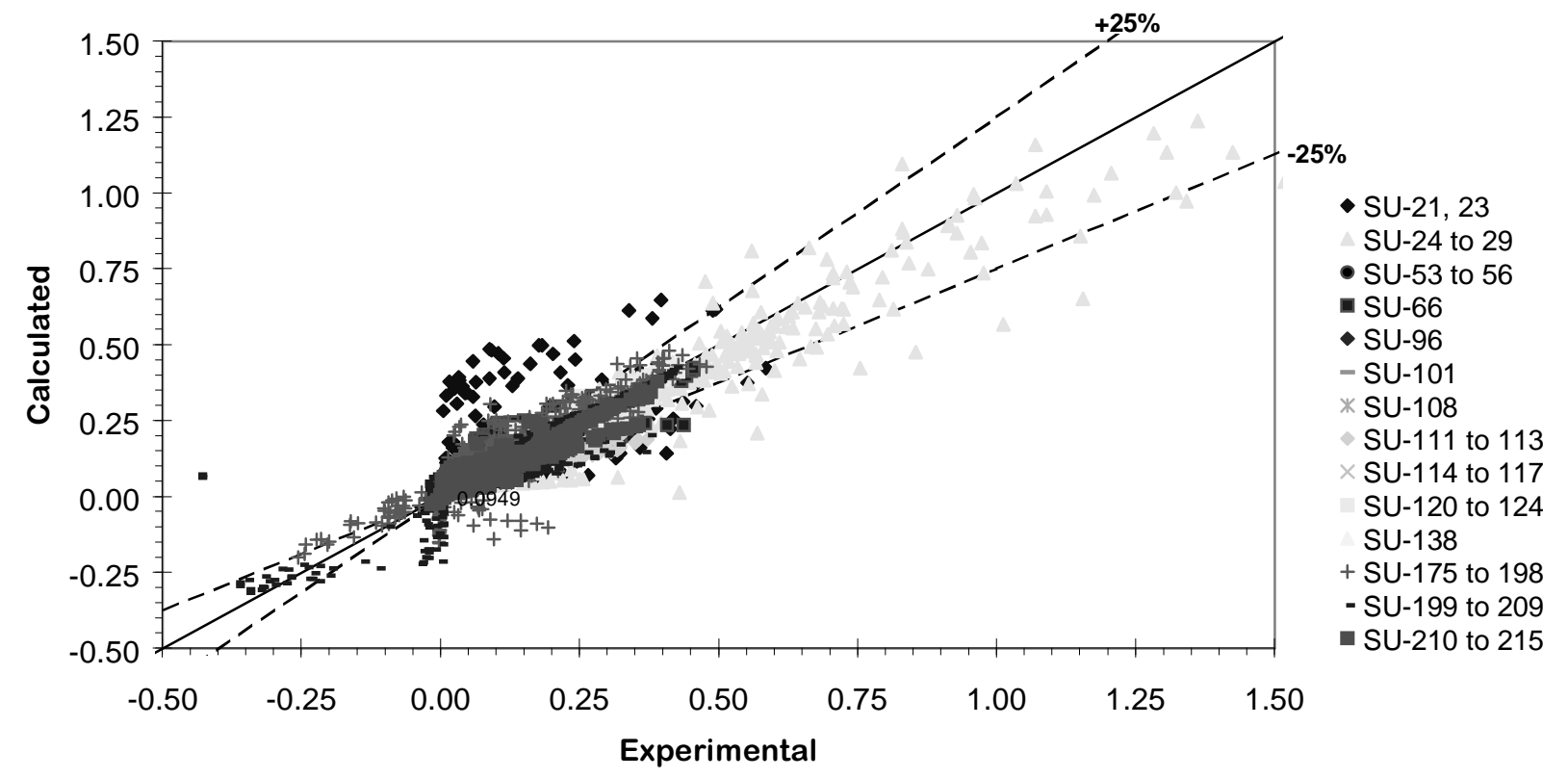

\section{FIGURE 2.3 - COMPARISON OF CALCULATED PRESSURE GRADIENT WITH EXPERIMENTAL DATA}

The total number of pressure gradient predictions to fall within $25 \%$ of the experimental observations improved slightly to 3,096 data points versus 2,977 for the previous version of the model. Similarly, the volume fraction liquid prediction resulted in a small improvement: 4,076 of the 5,951 data points were predicted to within $25 \%$ of the experimental data, up from 3,986 .

Table 2.1 shows how the new stratified model affects the flow pattern predictions. It is seen that further improvement is required here as the correct flow pattern is predicted in only 1,792 of the 5,951 cases. It should be noted, however, that intermittent flow (consisting of elongated bubble, slug, and froth flow) constitute the majority of the experimental observations and this model has yet to be optimized.

A further point to consider is that the stratified smooth and stratified wavy flow regimes are treated as a single flow pattern by the model. A distinction between the two is only made for the purposes of flow pattern prediction. If the transition between smooth and wavy stratified flow is ignored, it is seen that stratified flow was observed in 1,012 of the 5,951 cases. The model predicted stratified flow in 719 cases. Of these, the predictions matched the experimental observations in 464 cases, a significant improvement over the 110+92=202 reported in Table 2.1. This suggests that the transition mechanism between stratified smooth and wavy flow may need to be modified. 
TABLE 2.1 - FLOW PATTERN DISTRIBUTION IN EXPERIMENTAL DATA

\begin{tabular}{|lccc|}
\hline Flow Pattern & Predicted & Measured & Matched \\
\hline Elongated bubble & 394 & 584 & 106 \\
Bubble & 43 & 426 & 0 \\
Stratified smooth & 131 & 449 & 92 \\
Stratified wavy & 588 & 563 & 110 \\
Slug & 1511 & 2103 & 627 \\
Annular-Mist & 1416 & 1007 & 667 \\
Dispersed bubble & 498 & 411 & 38 \\
Froth I & 1323 & 408 & 152 \\
Froth II & 47 & 0 & 0 \\
\hline & & 5951 & 1792 \\
\hline
\end{tabular}

\subsection{Conclusions and Future Work}

The improvements to the stratified flow model have resulted in minor improvements to the mechanistic model's ability to predict flow patterns, pressure drop and holdup. There have also been significant reductions in the discontinuities between flow patterns resulting in much smoother transitions.

Significant additional improvements are expected to be achieved by optimizing the intermittent flow model in the same manner as has been done for annular-mist and for stratified flow. Once all of the individual flow patterns have been optimized, the transition mechanisms presently used in the model will be reassessed. The model will also be tested using data from actual wells (approximately 1,800 such measurements are available in the SMFD)..

Testing of the model with data from the Stanford horizontal well experiments has indicated that the accelerational effects due to inflow cannot be ignored. A model to account for these effects is being developed and when it is completed, it will be incorporated in and tested with the mechanistic model.

\section{Nomenclature}
A Cross-sectional area
D Pipe internal diameter
E In situ volume fraction
g Acceleration due to gravity
$\mathrm{h}_{\mathrm{L}} \quad$ Height of liquid (Stratified flow)
L Length
$\mathrm{p} \quad$ Pressure
Re Reynolds number
$\mathrm{S} \quad$ Contact perimeter 


$\begin{array}{ll}V_{S G} & \text { Superficial gas velocity } \\ V_{S L} & \text { Superficial liquid velocity } \\ \varepsilon_{\mathrm{k}} & \text { Pipe roughness } \\ \theta & \text { Angle of inclination } \\ \mu & \text { Viscosity } \\ \rho & \text { Density } \\ \sigma & \text { Interfacial (surface) tension } \\ \tau & \text { Shear stress } \\ \tilde{x} & \text { Dimensionless quantity, } x\end{array}$

Subscripts

$G \quad$ relating to the gas phase

$i \quad$ relating to the interface

$L \quad$ relating to the liquid phase

$S G \quad$ based on superficial gas velocity

$S L$ based on superficial liquid velocity

$w L$ relating to the wall-liquid interface

$w G$ relating to the wall-gas interface

\section{References}

1) Aziz, K. and Petalas, N., "New PC-Based Software for Multiphase Flow Calculations," paper SPE 28249 presented at the SPE Petroleum Computer Conference, Dallas, 31 July3 August, 1994

2) Baker, A., Nielsen, K. and Gabb A., "New Correlations - 1, Pressure Loss Liquid-Holdup Calculations Developed," Oil \& Gas J., March 14, 1988, 55-59

3) Colebrook, C. F., "Turbulent Flow in Pipes with Particular Reference to the Transition Region Between the Smooth and Rough-Pipe Laws,” J. Inst. Civil Engrs., 11, 133 (1939)

4) Petalas, N., and Aziz, K., "Stanford Multiphase Flow Database - User's Manual," Version 0.2, Petroleum Engineering Department, Stanford University.

5) Petalas, N., and Aziz, K., "Development and Testing of a New Mechanistic Model for Multiphase Flow in Pipes," Proceedings of the ASME Fluids Division Summer Meeting, Volume 1, FED-Vol. 236, pp. 153-159, July, 1996.

6) Petalas, N., and Aziz, K., "Development and Testing of Mechanistic Models for Multiphase Flow in Pipes - Phase I: A Preliminary Approach for Stabilized Flow at All Inclinations," Report for the Stanford Reservoir Simulation Industrial Affiliates and the Stanford Project on the Productivity and Injectivity of Horizontal Wells, Petroleum Engineering Department, Stanford University, April, 1996.

7) Xiao, J. J., Shoham, O., Brill, J. P., "A Comprehensive Mechanistic Model for TwoPhase Flow in Pipelines," paper SPE 20631, 65th ATC\&E of SPE, New Orleans, September 23-26, 1990 


\title{
3. Analysis of the 1995 and 1996 Stanford Horizontal Wellbore Experiments
}

\author{
by Liang-Biao Ouyang, Nicholas Petalas, Sepehr Arbabi, and Khalid Aziz
}

\begin{abstract}
A brief introduction to the 1996 Stanford Horizontal Wellbore experiments is presented. Different existing mechanistic models, simplified models as well as empirical correlations are applied to analyze the 1995 and 1996 Stanford horizontal wellbore experiments. Finally, comparisons between measurement and prediction are performed, which leads to the conclusion that no existing models can predict sufficiently accurate pressure drops for gas-liquid wellbore flow where wall mass transfer exists.
\end{abstract}

\subsection{Introduction}

Over the last decade horizontal wells have become a well established technology for the recovery of oil and gas. They have become increasingly attractive for production from thin reservoirs, naturally fractured reservoirs, reservoirs with gas and water coning problems, offshore environments where various wells are drilled from a central platform, as well as in enhanced oil recovery practices such as steam injection.

The modeling of horizontal wells poses additional challenges, one of which is the determination of the frictional pressure drop along the wellbore. The pressure drop due to friction is usually neglected in classical reservoir simulation models. However, practical examples have shown that in many cases the inclusion of wellbore friction is essential for the correct prediction of well performance. For horizontal wells, the frictional pressure drop becomes even more significant since the drilling technology now allows wells to be several thousands of feet long. Thus, the frictional pressure drop over the production portion of the well will be much larger in horizontal wells compared to that in vertical wells.

Depending on the completion method used in a horizontal well, fluid may enter the wellbore radially through perforations at various locations. This alters the flow behavior along the wellbore and complicates the modeling of the problem. For single phase flow, Ouyang et al. [1] have shown that influx through well perforations increases wall friction for the laminar flow regime and decreases wall friction for the turbulent flow regime. This means that the frictional pressure drop can be larger or smaller than that for no influx or no-wall-flow situations, depending on the flow regime present in the wellbore. In addition, as the distance between adjacent perforations may not be large enough to achieve a stabilized velocity profile, the flow characteristics and the pressure gradient in a horizontal wellbore may change with location. The flow behavior in a horizontal wellbore with an increasing flow rate along it due to influx and the relationship between pressure drop and influx from the reservoir are some of the most important but unsolved issues in the engineering analysis of horizontal wells. Furthermore, in certain cases, 
gas and liquid flow simultaneously in horizontal wellbores under different flow patterns. Since various flow patterns behave differently when no influx is present through the pipe walls, with inflow the problem will become more complicated.

A literature survey leads to the following observations:

- No correlations exist for determining wall friction factors for single phase and multiphase fluid flow in wellbores with inflow or outflow through perforations.

- The accelerational and inflow-directional pressure drops are neglected in most of the single wellbore flow models or wellbore-reservoir coupling models.

- The wall friction shear is usually evaluated by using the friction factor correlations for pipe flow without mass transfer through pipe wall. Therefore, the impact of mass transfer through the pipe wall on the wall friction is not included.

- No mechanistic model has been developed to predict the flow characteristics, such as flow pattern, liquid holdup and pressure drop in multiphase wellbore flow with the presence of inflow or outflow through perforations.

In order to investigate the influence of inflow on single phase and multiphase wellbore flow behavior, experiments have been designed and performed by Stanford University in 1994 for oil-nitrogen and in 1995 for air-water flow in a 100 feet-long horizontal wellbore model at the Marathon Oil Company's Littleton facility. For 1996, the wellbore model was modified by inserting stainless steel wire-wrapped screens inside the base acrylic pipe. Air-water flow experiments, similar to the 1995 experiments, were carried out using this setup, as described in more detail in the next section.

\subsection{Experimental Setup}

The horizontal wellbore flow model is shown in Figure 3.1. The outer acrylic pipe is the same as used during the 1995 experiments. It is 100 feet long and consists of twenty 5-feet sections with an average internal diameter of 6.2 inches. The longer inner pipe is made of three acrylic window sections (denoted by $\mathrm{W}$ in Figure 3.1), each about 4 feet long, and three stainless steel wire-wrapped screen sections, each about 21 feet long. Packers are used to eliminate flow in the annular space around the window sections. Inflow only occurs in the screen sections so that the total inflow length is approximately 63 feet, down from 75 feet in the 1995 experiments. Window sections are used to observe and record the flow regimes and they each house a nuclear densitometer to measure the average in situ liquid fraction. Figure 3.2 shows the average measured internal diameter of different sections of the inner wellbore. The screen sections have a very small variation in diameter (4.500 to $4.509 \mathrm{in})$, whereas the window sections show a somewhat larger variation in their diameters (4.450 to $4.476 \mathrm{in})$. However, both variations are much smaller than the variations in diameters of the sections forming the outer pipe.

A total of 164 experiments have been conducted (Table 3.1) which can be classified as:

- Single phase water flow without inflow - calibration experiment (series 0 and V)

- Single phase water axial flow with water inflow (series IA1-IA3 and IV)

- Air and water two-phase flow 
1. Air-water axial flow without inflow (series IIA1-IIA3)

2. Water axial flow with air inflow (series IIB1-IIB3)

3. Air-water axial flow with single phase inflow

- Water inflow (series IIC1a-IIC1c)

- Air Inflow (series IIC2a-IIC2b)

4. Air and water axial flow with air and water inflow (series IID1a-IID3b)

For each experimental run, axial flow and inflow flow rates; the liquid fractions at 11.25 feet, 36.25 feet, and 61.25 feet locations; pressure drops every 10 feet and 50 feet; pipe inclination angle; inlet and outlet temperatures and pressures were measured and recorded automatically into a Microsoft Excel file for later analyses. For most experiments, data were recorded for at least 10 minutes after reaching stabilized flow.

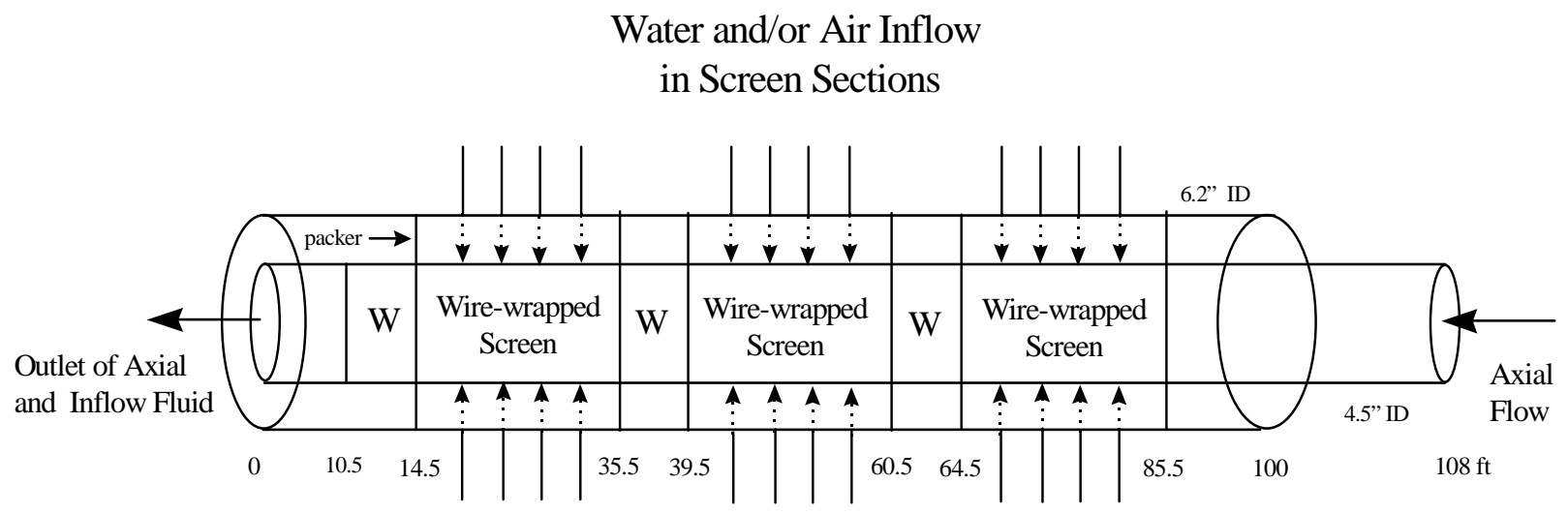

Figure 3.1: Wellbore Section Used for the 1996 Horizontal Flow Experiments

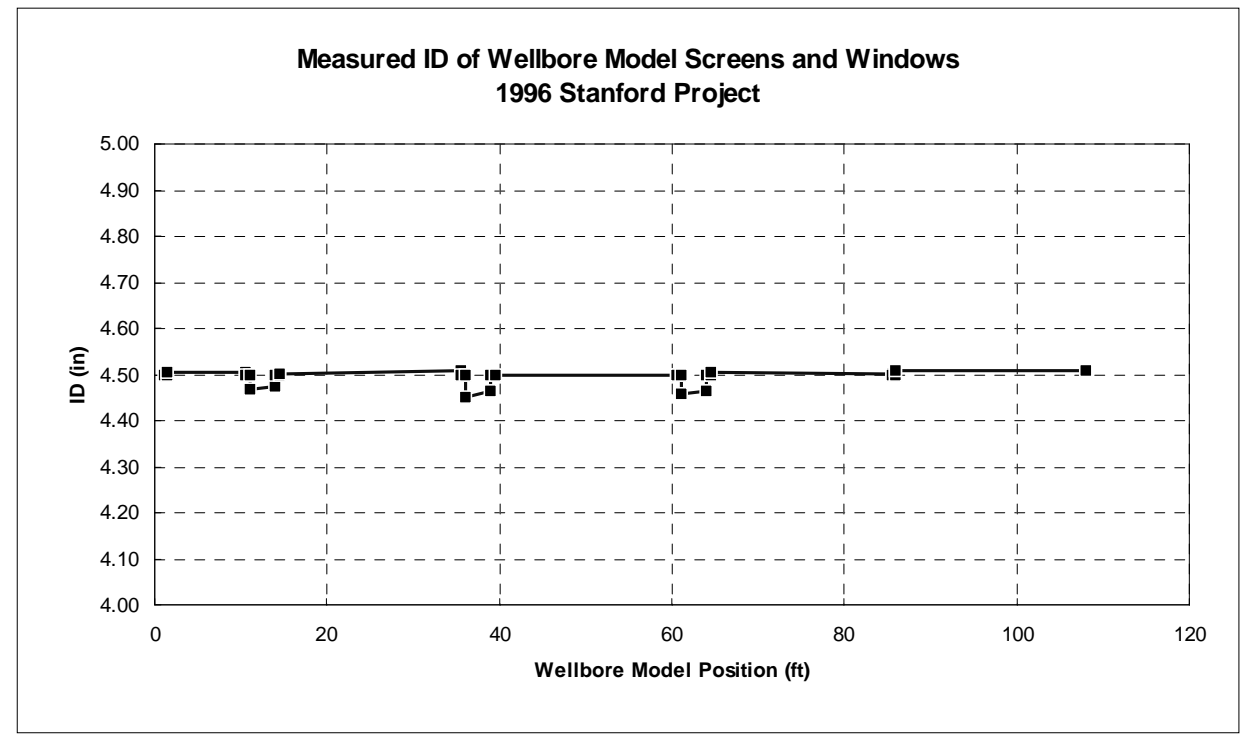

Figure 3.2: Average Measured Wellbore Internal Diameter for Each Section 
Table 3.1: Experiments Conducted in 1996

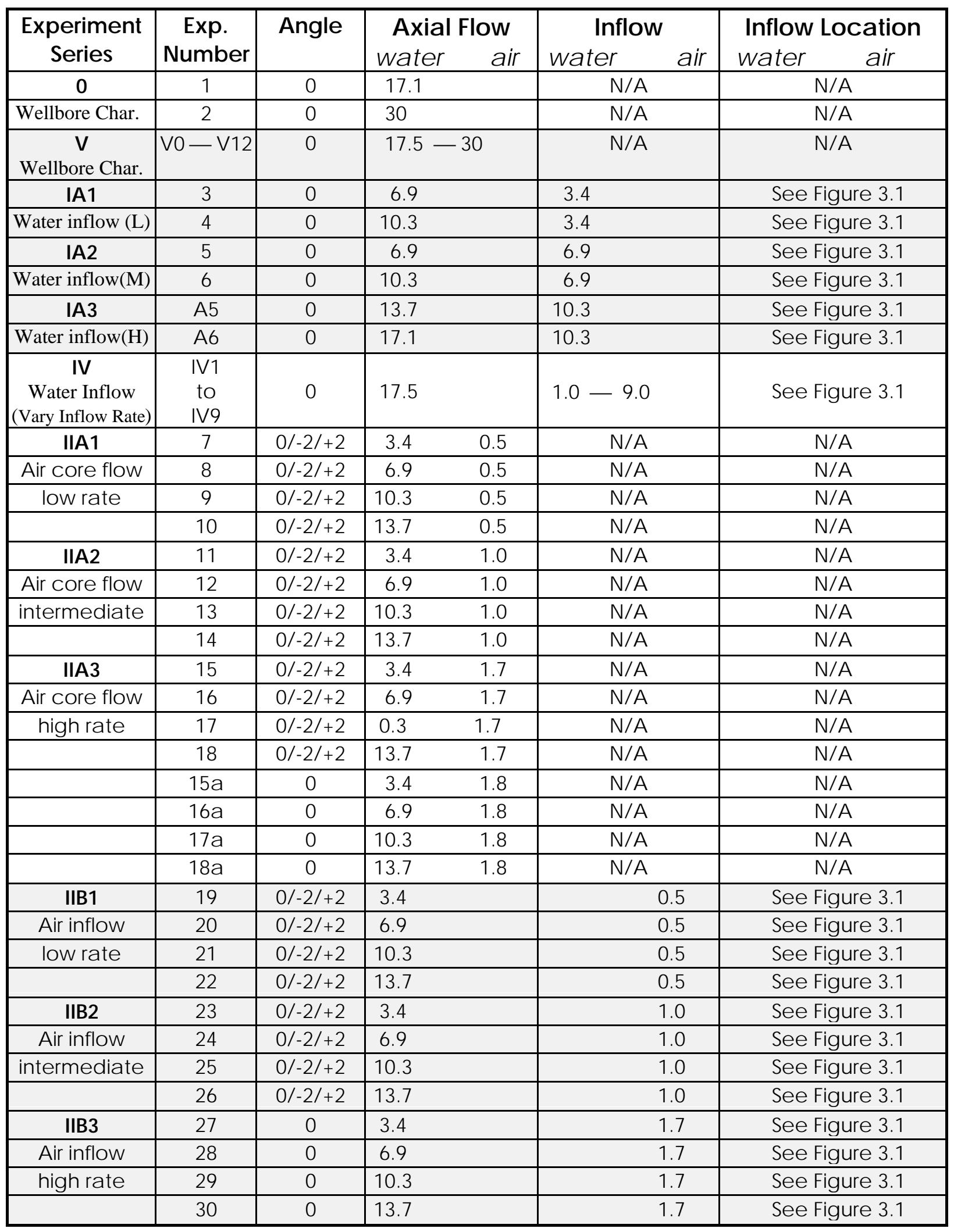


Table 3.1: Experiments Conducted in 1996 (continued)

\begin{tabular}{|c|c|c|c|c|c|c|c|}
\hline \multirow{2}{*}{$\begin{array}{l}\text { Expeniment } \\
\text { Series }\end{array}$} & \multirow{2}{*}{\begin{tabular}{|c|} 
Exp. \\
Number
\end{tabular}} & \multirow[t]{2}{*}{ Angle } & \multicolumn{2}{|c|}{ Axial fow } & \multicolumn{2}{|c|}{ Infiow } & Inflow Location \\
\hline & & & water & air & water & air & water \\
\hline IICla & 31 & $0 /-2 /+2$ & 3.4 & 0.5 & 3.4 & & See Figure 3.1 \\
\hline Aircore flow & 32 & $0 /-2 /+2$ & 6.9 & 0.5 & 3.4 & & See Figure 3.1 \\
\hline \multirow[t]{2}{*}{ water inflow } & 33 & $0 /-2 /+2$ & 10.3 & 0.5 & 3.4 & & See Figure 3.1 \\
\hline & 34 & $0 /-2 /+2$ & 13.7 & 0.5 & 3.4 & & See Figure 3.1 \\
\hline IIC 1b & 35 & $0 /-2 /+2$ & 3.4 & 1.0 & 3.4 & & See Figure 3.1 \\
\hline Air core flow & 36 & $0 /-2 /+2$ & 6.9 & 1.0 & 3.4 & & See Figure 3.1 \\
\hline \multirow[t]{2}{*}{ water inflow } & 37 & $0 /-2 /+2$ & 10.3 & 1.0 & 3.4 & & See Figure 3.1 \\
\hline & 38 & $0 /-2 /+2$ & 13.7 & 1.0 & 3.4 & & See Figure 3.1 \\
\hline IIC IC & 39 & 0 & 3.4 & 1.7 & 3.4 & & See Figure 3.1 \\
\hline Air core flow & 40 & 0 & 6.9 & 1.7 & 3.4 & & See Figure 3.1 \\
\hline \multirow[t]{6}{*}{ water inflow } & 41 & 0 & 10.3 & 1.7 & 3.4 & & See Figure 3.1 \\
\hline & 42 & 0 & 13.7 & 1.7 & 3.4 & & See Figure 3.1 \\
\hline & $39 a$ & 0 & 3.4 & 1.8 & 3.4 & & See Figure 3.1 \\
\hline & $40 a$ & 0 & 6.9 & 1.8 & 3.4 & & See Figure 3.1 \\
\hline & $41 a$ & 0 & 0.3 & 1.8 & 3.4 & & See Figure 3.1 \\
\hline & $42 a$ & 0 & 13.7 & 1.8 & 3.4 & & See Figure 3.1 \\
\hline IIC2a & 43 & $0 /-2 /+2$ & 3.4 & 0.5 & & 0.5 & See Figure 3.1 \\
\hline Air core flow & 44 & $0 /-2 /+2$ & 6.9 & 0.5 & & 0.5 & See Figure 3.1 \\
\hline \multirow[t]{2}{*}{ air inflow } & 45 & $0 /-2 /+2$ & 10.3 & 0.5 & & 0.5 & See Figure 3.1 \\
\hline & 46 & $0 /-2 /+2$ & 13.7 & 0.5 & & 0.5 & See Figure 3.1 \\
\hline IIC 2b & 47 & $0 /-2 /+2$ & 3.4 & 0.5 & & 1.0 & See Figure 3.1 \\
\hline Air core flow & 48 & $0 /-2 /+2$ & 6.9 & 0.5 & & 1.0 & See Figure 3.1 \\
\hline \multirow[t]{2}{*}{ a ir inflow } & 49 & $0 /-2 /+2$ & 10.3 & 0.5 & & 1.0 & See Figure 3.1 \\
\hline & 50 & $0 /-2 /+2$ & 13.7 & 0.5 & & 1.0 & See Figure 3.1 \\
\hline \multirow[t]{2}{*}{ IIDla } & 51 & 0 & 3.4 & 0.5 & 3.4 & 0.5 & See Figure 3.1 \\
\hline & 52 & 0 & 13.7 & 0.5 & 3.4 & 0.5 & See Figure 3.1 \\
\hline \multirow[t]{2}{*}{ IID1b } & 53 & 0 & 3.4 & 0.5 & 3.4 & 0.5 & See Figure 3.1 \\
\hline & 54 & 0 & 13.7 & 0.5 & 3.4 & 0.5 & See Figure 3.1 \\
\hline \multirow[t]{2}{*}{ IID2a } & 55 & 0 & 3.4 & 0.5 & 3.4 & 0.5 & See Figure 3.1 \\
\hline & 56 & 0 & 13.7 & 0.5 & 3.4 & 0.5 & See Figure 3.1 \\
\hline \multirow[t]{2}{*}{ IID2b } & 57 & 0 & 3.4 & 0.5 & 3.4 & 0.5 & See Figure 3.1 \\
\hline & 58 & 0 & 13.7 & 0.5 & 3.4 & 0.5 & See Figure 3.1 \\
\hline \multirow[t]{2}{*}{ IID3a } & 59 & 0 & 3.4 & 0.5 & 3.4 & 0.5 & See Figure 3.1 \\
\hline & 60 & 0 & 13.7 & 0.5 & 3.4 & 0.5 & See Figure 3.1 \\
\hline \multirow[t]{2}{*}{ IID3b } & 61 & 0 & 3.4 & 0.5 & 3.4 & 1.0 & See Figure 3.1 \\
\hline & 62 & 0 & 13.7 & 0.5 & 3.4 & 1.0 & See Figure 3.1 \\
\hline Total & 164 & & & & & & \\
\hline
\end{tabular}




\subsection{Analysis of Experimental Data}

\subsubsection{Overview}

Preliminary comparison results of the 1995 Stanford horizontal wellbore experiments were given in the 1996 Annual Report to the Department of Energy (DOE) of the United States [2]. Due to time limitation, only part of the 1995 experimental data was analyzed and compared with predictions by simplified models, mechanistic models as well as emprirical correlations. In the past year, research associated with the Stanford horizontal wellbore experiments includes: (a) Complete analysis for both 1995 and 1996 experiments; (b) Improvement of the Stanford mechanistic model for gas-liquid two-phase flow in pipes; (c) Development of new models (including a simplified model and mechanistic models) and correlations to account for mass transfer through wellbore perforations. Results from the first area of our research are presented

\subsubsection{Model Prediction Procedure}

In all, five models are selected for comparison with experimental data:

- the Stanford mechanistic model (Petalas \& Aziz, 1996 [3]);

- the Xiao, Shoham \& Brill model (1990) [4];

- the Beggs \& Brill model (1973, revised 1977) [5];

- the Dukler, Wicks \& Cleveland (1964) correlation [6];

- the no-slip homogeneous model (Beattie \& Whalley, 1982 [7]).

Among these models, the first three involve a mechanistic approach, the fourth is an empirical correlation, while the last one is a simplified flow model. Note that the models or correlations used do not account for the effects of mass transfer through the pipe wall on wall friction and on the kinetic energy change.
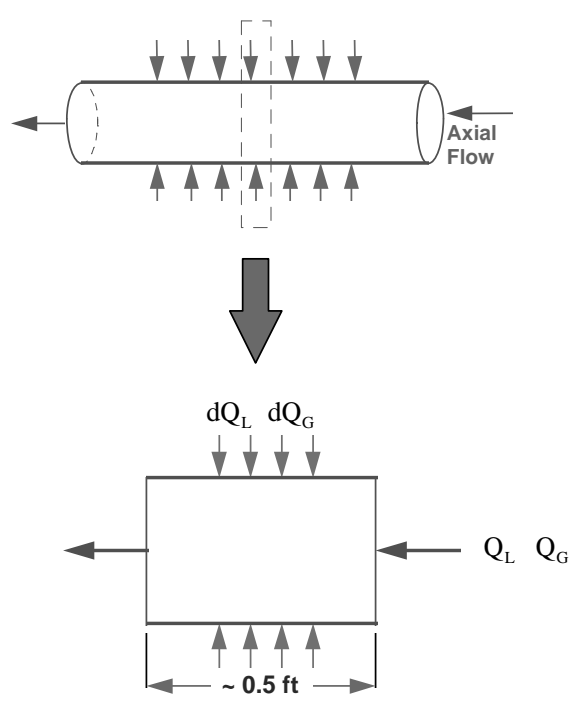

Sum up to get the information of flow pattern change along the whole pipe and the pressure drop over any segment of interest

Figure 3.3: Segmented Model Approach 
Because of inflow through the perforations, the local gas and liquid flow rates (thus superficial velocities $U_{S L}$ and $U_{S G}$ ) vary with wellbore location. Consequently, a segmented model approach as shown in Figure 3.3 must be applied. This approach features three primary steps:

1). Divide the entire wellbore into small segments such that the predicted pressure drop along each segment is smaller than a specified tolerance.

2). Compute the gas and liquid flow rates entering each segment through the perforations and add these inflow rates to the axial flow rates at the inlet of the segment.

3). Determine the flow pattern, if applicable (based on the model being used), and the pressure drop along each segment and sum them up to obtain pressure drops along the entire wellbore or along any section of interest.

\subsubsection{Comparison between Measurement and Prediction}

\section{Experiments}

A total of 134 experimental runs featuring different combinations of axial flow and influx were conducted in the 1995 program [2], including

- $\quad$ single phase water axial flow with water inflow;

- single phase water axial flow with air inflow;

- air and water two-phase axial flow with water inflow;

- air and water two-phase axial flow with air inflow;

- air and water two-phase axial flow and inflow.

Some duplicate experiments were performed, which increases the total number of experimental runs to 200. Among all these experiments, more than $95 \%$ fall into air-water two-phase flow category and will be used in the comparison below.

Comparisons between measured and predicted pressure drops for the 1995 experiments are shown in Figures 3.4-3.8. Two plots are included in each figure, where plot (a) compares the pressure drop along the section of pipe from 40 to 50 feet, while plot (b) shows the comparison of the pressure drop along the entire wellbore (100 feet long). The following observations can be made based on Figures 3.4-3.8:

1). Large scatter is seen in Figures 3.4-3.8, indicating that there exist significant differences between experimental data and model predictions. To make things worse, no apparent trends are evident in the data, except that all the models overpredict the pressure drop for downward flow. For horizontal and upward flow, the models overpredict pressure drop for some runs, while underpredicting for others. 
2). It is found that predictions by the Stanford mechanistic model improve by either ignoring stratified flow or forcing annular-mist flow. Particularly, significant improvements in prediction are found in the case of forcing annular-mist flow, though nontrivial discrepancies are still present in this case.

3). The predicted pressure drops along the entire wellbore and the section at 40-50 feet by the Xiao, Shoham \& Brill (1990) model [4] become zero at numerous data points (Figure 3.5). Horizontal pipe flow occurs for most of these data points. The underlying reason is that the Xiao, Shoham \& Brill (1990) model [4] predicts negative values of pressure drop for these experiments, which is obviously incorrect.

Figures 3.9-3.13 are similar to those listed in Figures 3.4-3.8. The only difference is that the data points are classified based on influx in Figures 3.9-3.13 as opposed to inclination angle. All the data points are divided into four groups: (a) no influx; (b) air influx only; (c) water influx only; (d) both air and water influx. Basically, all the models reflected in Figures 3.9-3.13, were developed for gas-liquid two-phase pipe flow without any influx (we refer to this type of flow as regular pipe flow to distinguish it from pipe flow with wall mass transfer). Unfortunately, the predictions for regular pipe flow based on these models deviate substantially from measurements. One of the possible reasons is that the closure relationships or correlations used in these models were proposed on the basis of experimental data with smaller pipe diameters and therefore may introduce some inaccuracy for large pipe diameter situations such as in the 1995 Stanford experiments. Another possible reason is that pressure drops were not very high, which makes accurate measurement difficult. The models also seem to overpredict pressure drop for air-water two-phase flow without influx (Figures 3.9-3.13). This could indicate that an inappropriate pipe roughness has been used.

\section{Experiments}

The 1996 Stanford horizontal wellbore experiments differ from the 1995 experiments primarily in wellbore geometry and inflow format. The average wellbore internal diameter is 4.5 inch in 1996 as compared to 6.18 inch in 1995. Fluid (air and/or water) influx is through a screen in 1996 experiments, whereas it is through perforations, uniformly distributed over the wellbore test section, in 1995 (Ouyang et al. [2]). Excluding 52 single phase experiments, there are 162 experimental runs related to air-water two-phase flow if repeated measurements are also counted. As in the analysis for the 1995 experiments, the same models are applied to predict pressure drop along the entire pipe length and comparisons between model predictions and the experimental data are shown in Figure 3.14-3.23. Due to the smaller pipe internal diameter, measured pressure drops are much higher than for experiments in 1995 with the same air and water flow rates. Greater than 10 psia pressure drops along the whole pipe were observed in nearly half of the experimental runs.

As before, the data points are grouped according to pipe inclination angle in Figures 3.14-3.18, while they are grouped by influx in Figures 3.19-3.23. From these figures it is 
observed that the model predictions for the 1996 Stanford experiments are much improved over those for the 1995 experiments and less data scatter is seen. The following observations can be made:

1) The Stanford mechanistic model provides good predictions for some of the experimental runs (Figure 3.14), although significant differences still exist in other parts of the data. It is interesting to note that for most of the data points, the Stanford mechanistic model substantially overpredicts pressure drop during downward flow. The predictions improve if either stratified flow is ignored or annular-mist flow is forced. Even in these cases, the Stanford mechanistic model slightly underpredicts the pressure drop.

2) The pressure drop predictions for the 1996 experiments by the Xiao, Shoham \& Brill model, the Dukler, Wicks \& Cleveland correlation and the no-slip homogeneous model are similar and they all show a remarkable improvement over the predictions for the 1995 experiments (Figures 3.15, 3.17 and 3.18). For a majority of data points, however, all these models underpredict the pressure drop. It is apparent that improvement in these models is necessary in order to apply them to wellbore flow with influx.

3) The worst predictions of pressure drop for the 1996 experiments are observed for the Beggs \& Brill model (Figure 3.16), although, here too, predictions show an improvement over those for the 1995 experiments.

The comparisons shown in Figures 3.19-3.23, where data was grouped by influx, are interesting. Fairly clear trends in model predictions can be seen:

1) All the models underpredict the pressure drop for cases with gas influx. They either slightly overpredict the pressure drop or give good predictions for cases without influx or with water influx.

2) Both the Stanford mechanistic model and the no-slip homogeneous model result in good predictions for cases with no influx.

3) The no-slip homogeneous model and the Xiao, Shoham \& Brill model are able to provide fairly accurate predictions for most experimental runs with water influx.

\subsection{Discussions \& Conclusions}

A segmented method has been applied to predict flow pattern, pressure drop and liquid holdup by using existing mechanistic models or empirical correlations. Unfortunately, substantial differences between measurements and predictions have been observed. Some of the possible reasons are given below: 
- Existing models or correlations do not account for the inflow effect, although the kinetic energy effect can be quite significant due to inflow.

- Correlations for wall friction factors, interfacial friction factor and liquid entrainment fraction were proposed for gas-liquid two-phase pipe flow without inflow, as a consequence they may not be appropriate for air and water wellbore flow where inflow occurs.

- Some transition criteria in existing mechanistic models may be inappropriate for wellbore flow with influx, and the flow patterns predicted may be incorrect.

\section{References}

[1] Ouyang, L-B., Arbabi, S., and Aziz, K. "General Single Phase Wellbore Flow Model for Horizontal, Vertical and Slanted Well Completions," SPE paper 36608, presented at the 71st Annual SPE Technical Conference \& Exhibition, Denver, Colorado, 1996

[2] Ouyang, L-B., Arbabi, S., and Aziz, K. "Preliminary Analysis of the 1995 Stanford Horizontal Wellbore Experiments," In Productivity and Injectivity of Horizontal Wells, Annual Report for Department of Energy (DOE) of U. S., Stanford University, pp1-24, 1996

[3] Petalas, N., and Aziz, K. "Development and Testing of a New Mechanistic Model for Multiphase Flow in Pipes," Second International Symposium on Numerical Methods for Multiphase Flows, ASME Fluids Engineering Division Summer Meeting, July 7-11, 1996, San Diego, California, USA.

[4] Xiao, J. J., Shoham, O. and Brill, J. P. "A Comprehensive Mechanistic Model for TwoPhase Flow in Pipelines," SPE paper 20631, presented at the 65th SPE Annual Technical Conference \& Exhibition, New Orleans, LA, 1990

[5] Beggs, H. D., and Brill, J. P. "A Study of Two-Phase Flow in Inclined Pipes," Trans. AIME, vol 256, 607, 1973.

[6] Dukler, A. E., Wicks, M., and Cleveland, R. G. "Frictional Pressure Drop in Two Phase Flow: A. A Comparison of Existing Correlations for Pressure Loss and Holdup," AIChE J., vol 10, 38, 1964.

[7] Beattie, D. R. H., and Whalley, P. B. "A Simple Two-Phase Frictional Pressure Drop Calculation Method," Int. J. Multiphase Flow, vol 8, no 1, 83-87, 1982. 


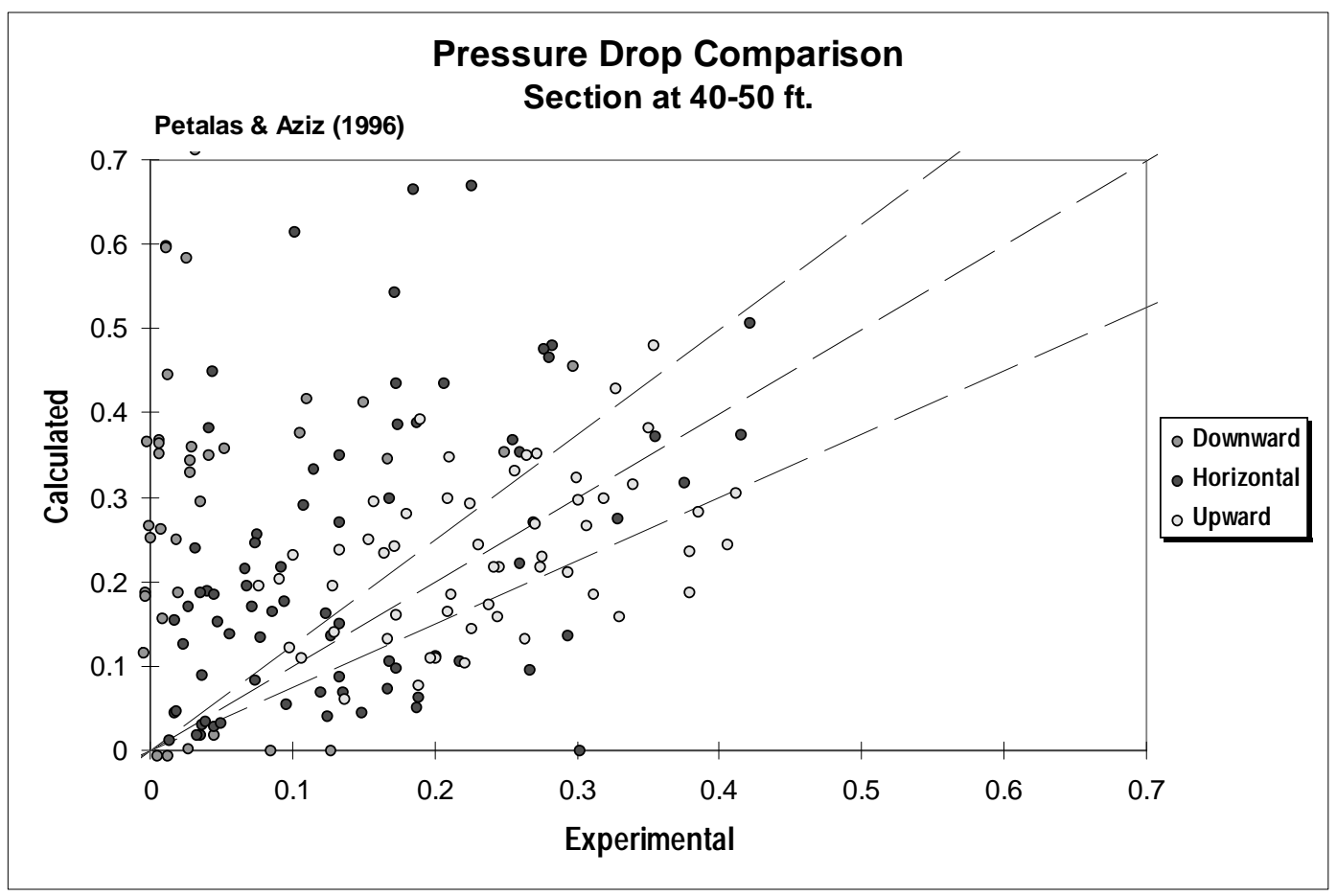

(a) Pressure Drop along the Section at 40-50 feet

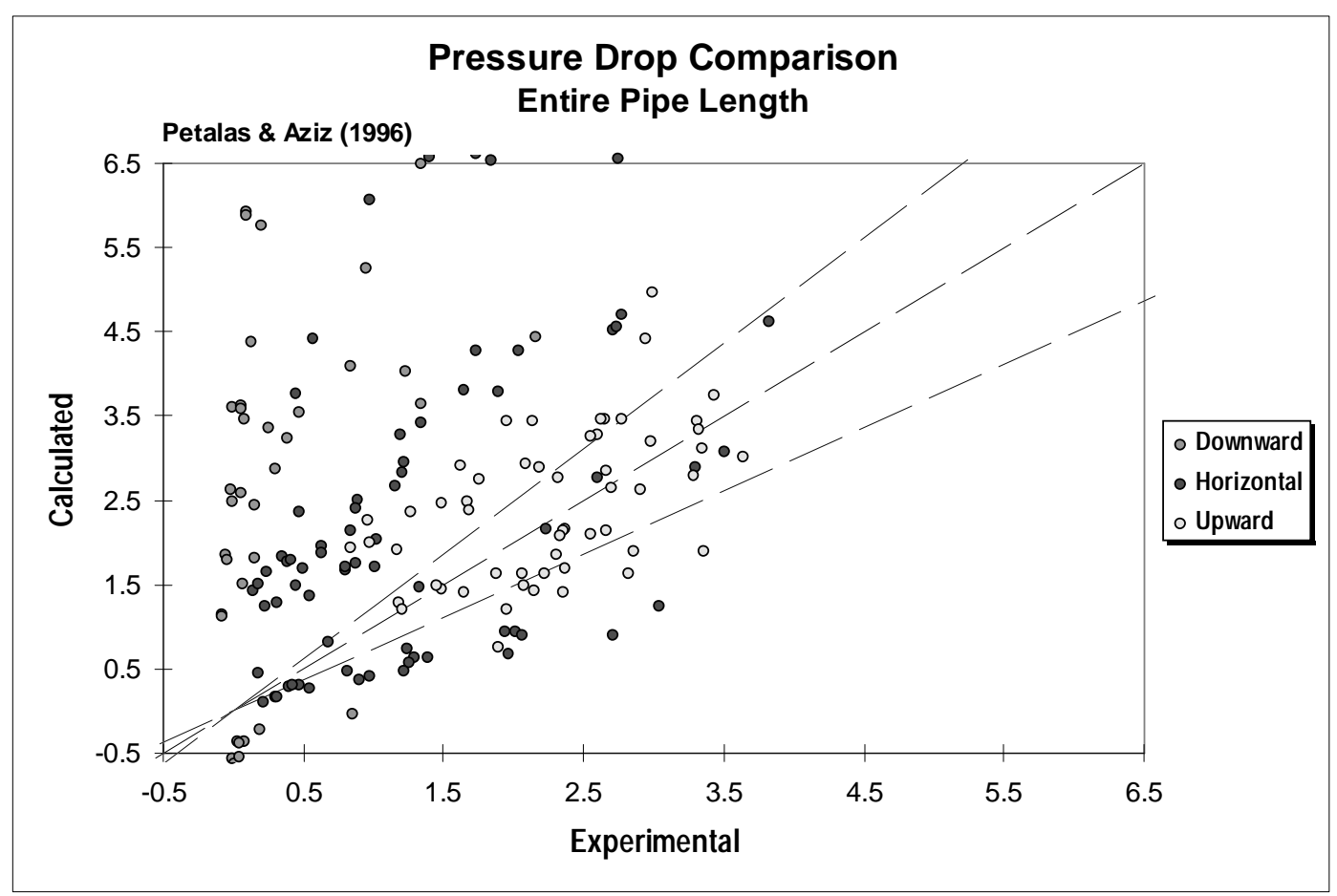

(b) Entire Pressure Drop

Figure 3.4 Comparison between Measured Pressure Drop and Prediction by Petalas \& Aziz (1996) Model (1995 Experiments) 


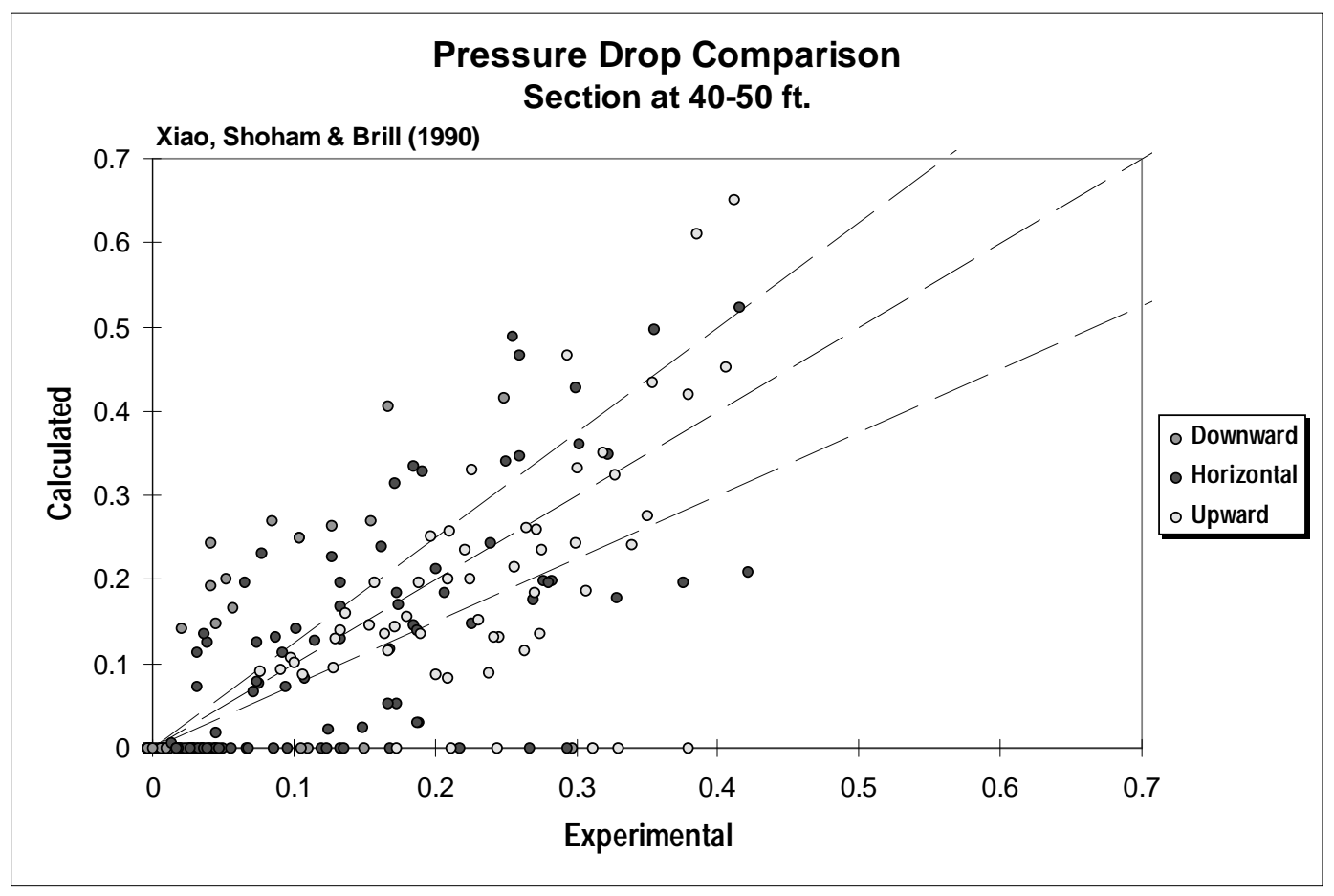

(a) Pressure Drop along the Section at 40-50 feet

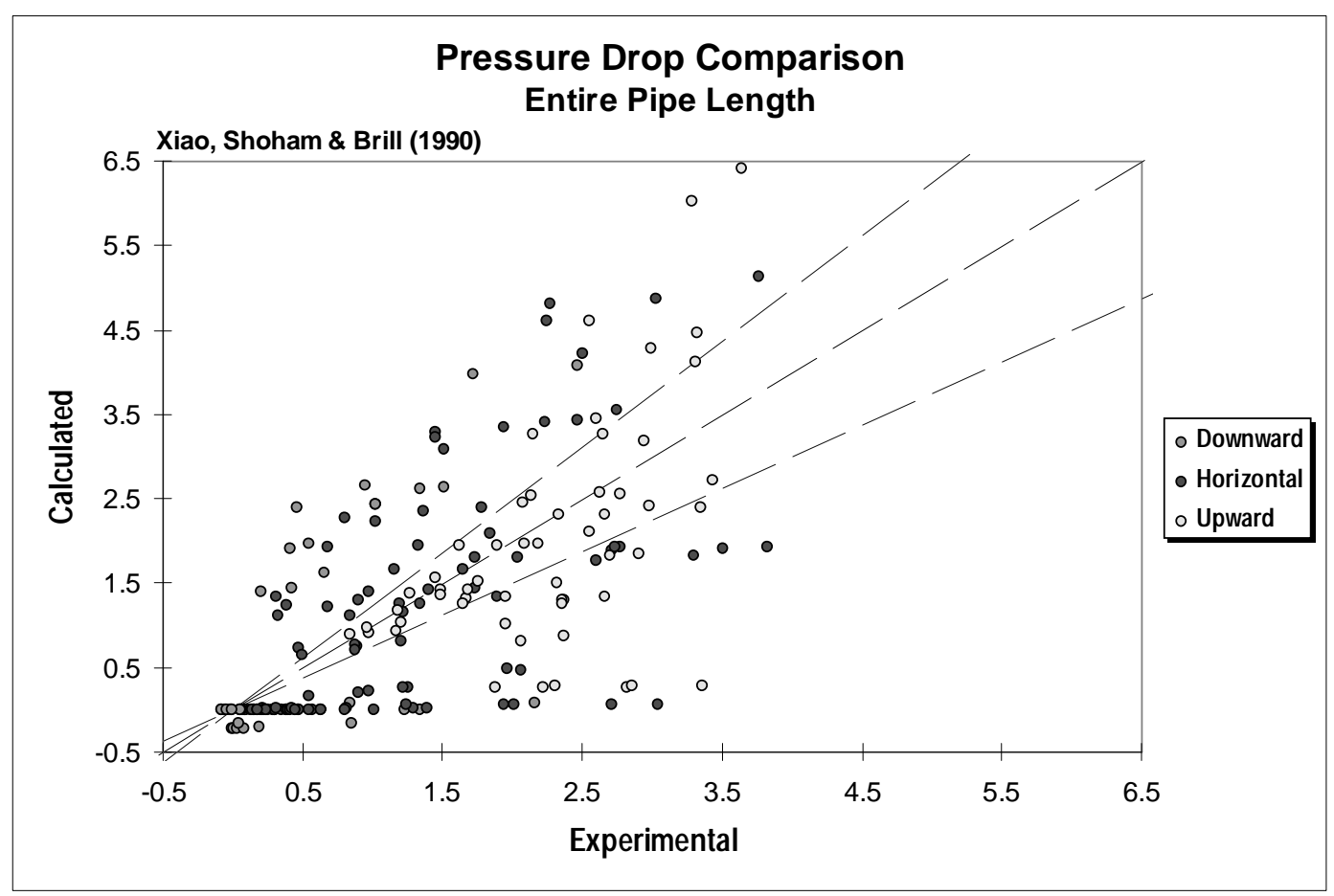

(b) Entire Pressure Drop

Figure 3.5 Comparison between Measured Pressure Drop and Prediction by Xiao, Shoham \& Brill (1990) Model (1995 Experiments) 


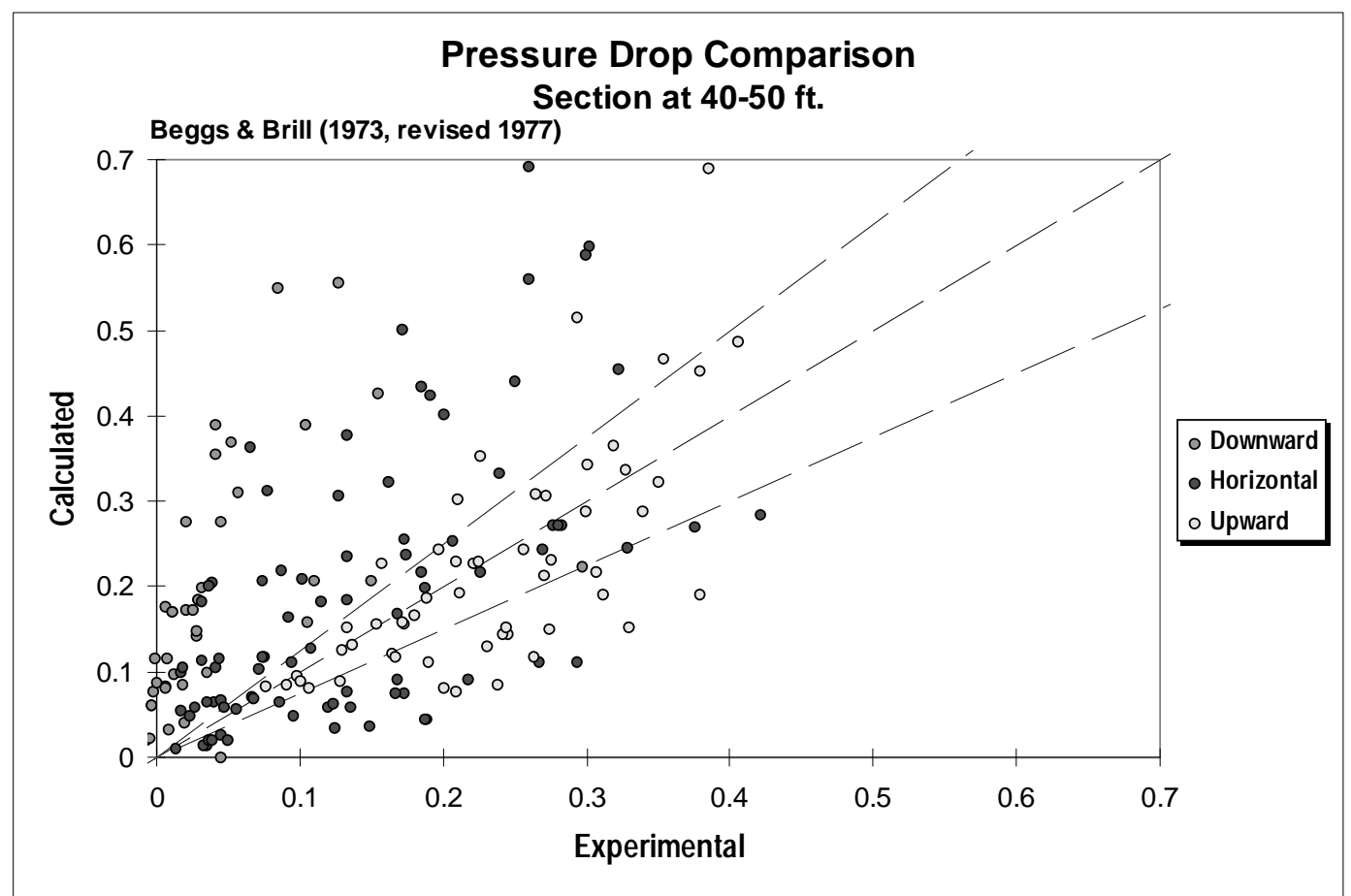

(a) Pressure Drop along the Section at 40-50 feet

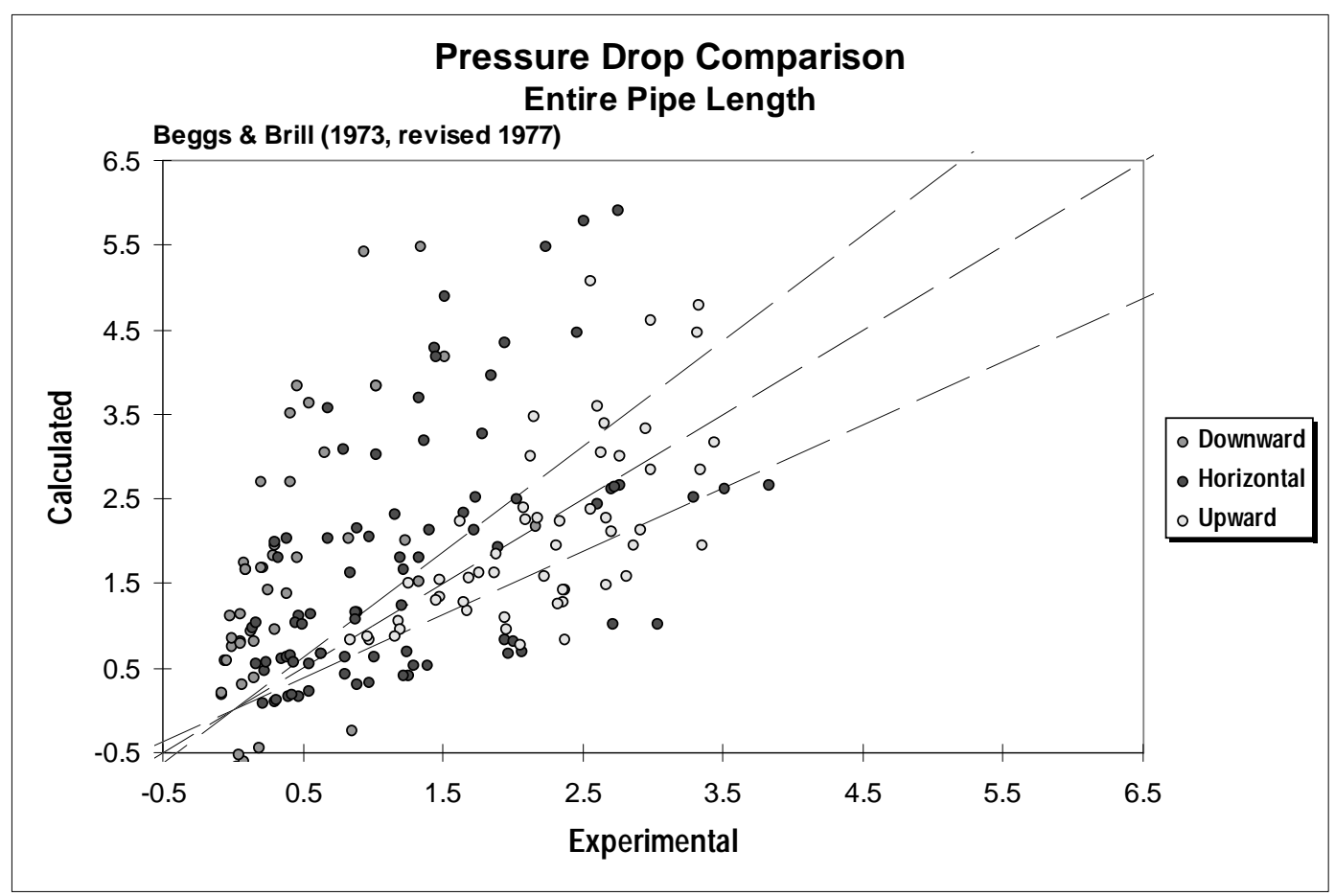

(b) Entire Pressure Drop

Figure 3.6 Comparison between Measured Pressure Drop and Prediction by Beggs \& Brill (1973, revised 1977) Model (1995 Experiments) 


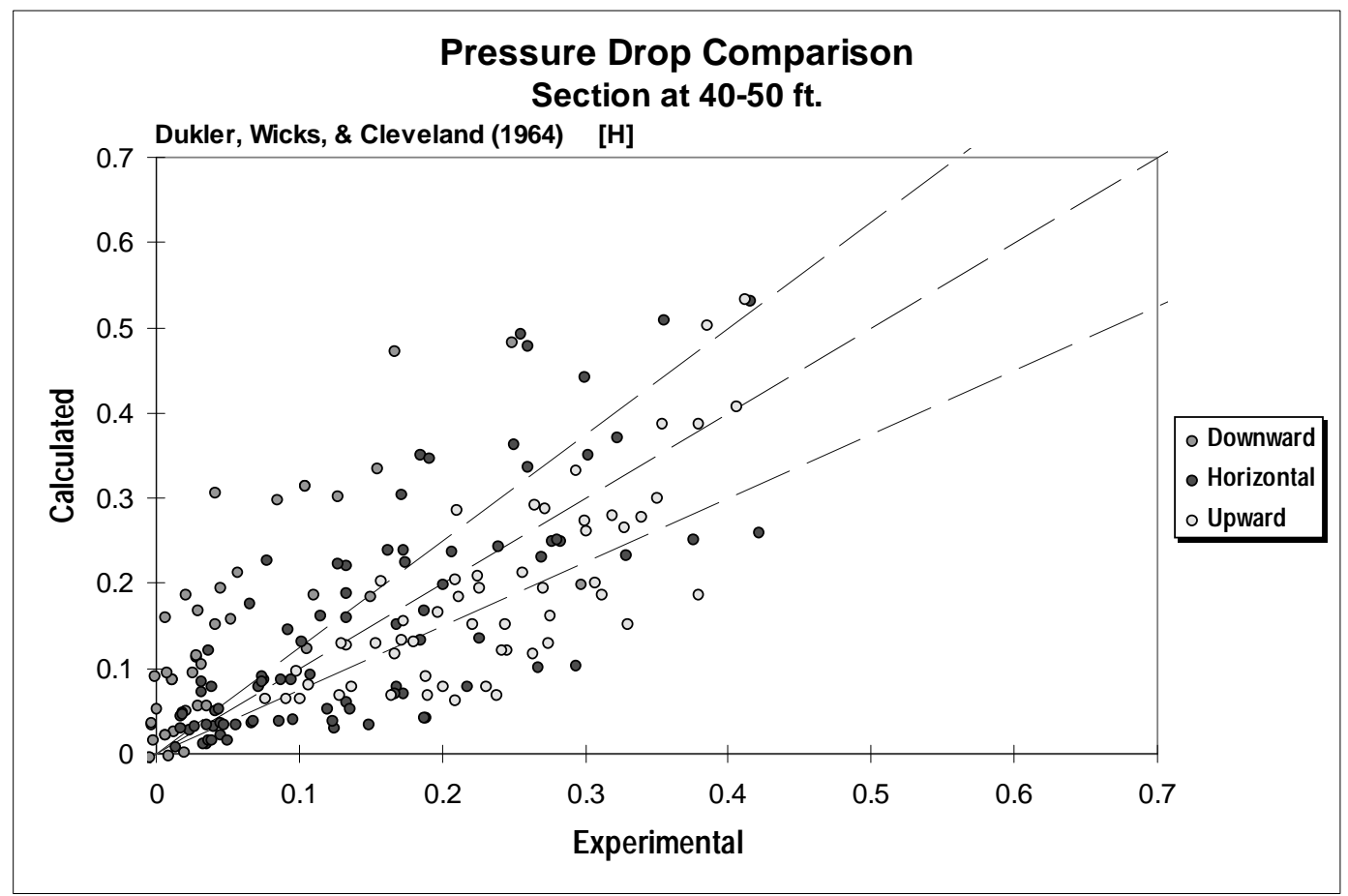

(a) Pressure Drop along the Section at 40-50 feet

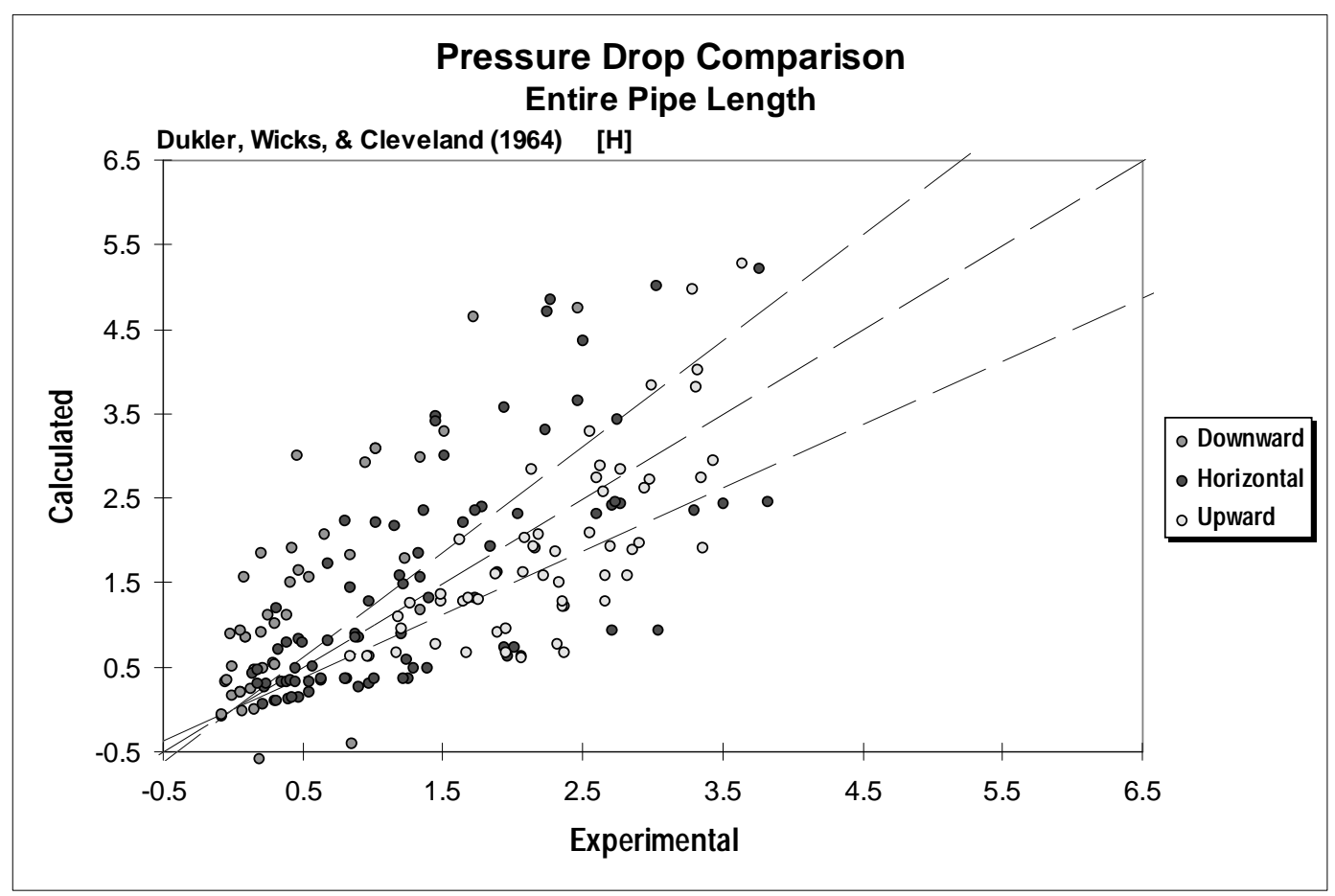

(b) Entire Pressure Drop

Figure 3.7 Comparison between Measured Pressure Drop and Prediction by Dukler, Wicks \& Cleveland (1964) Correlation (1995 Experiments) 


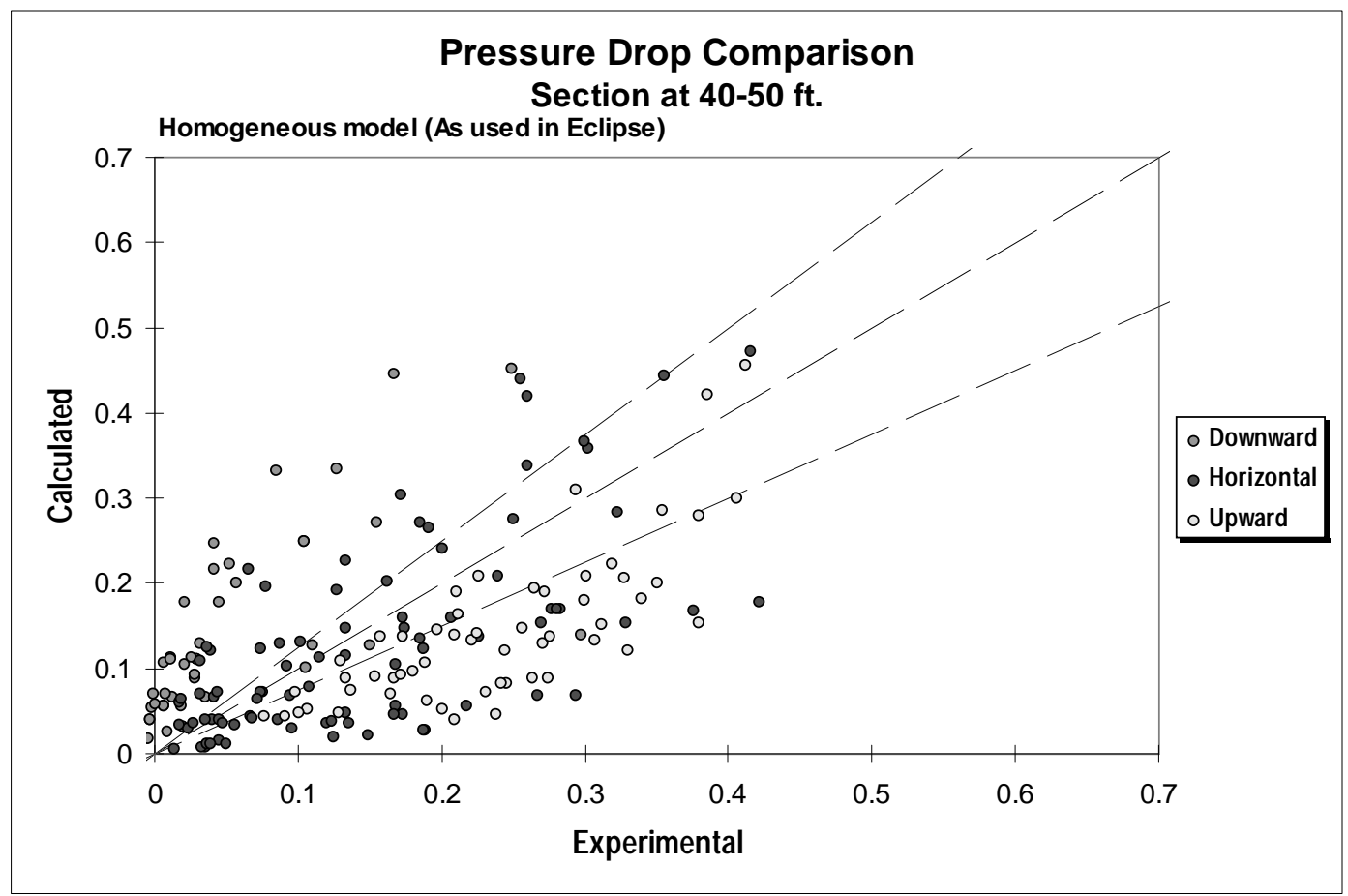

(a) Pressure Drop along the Section at 40-50 feet

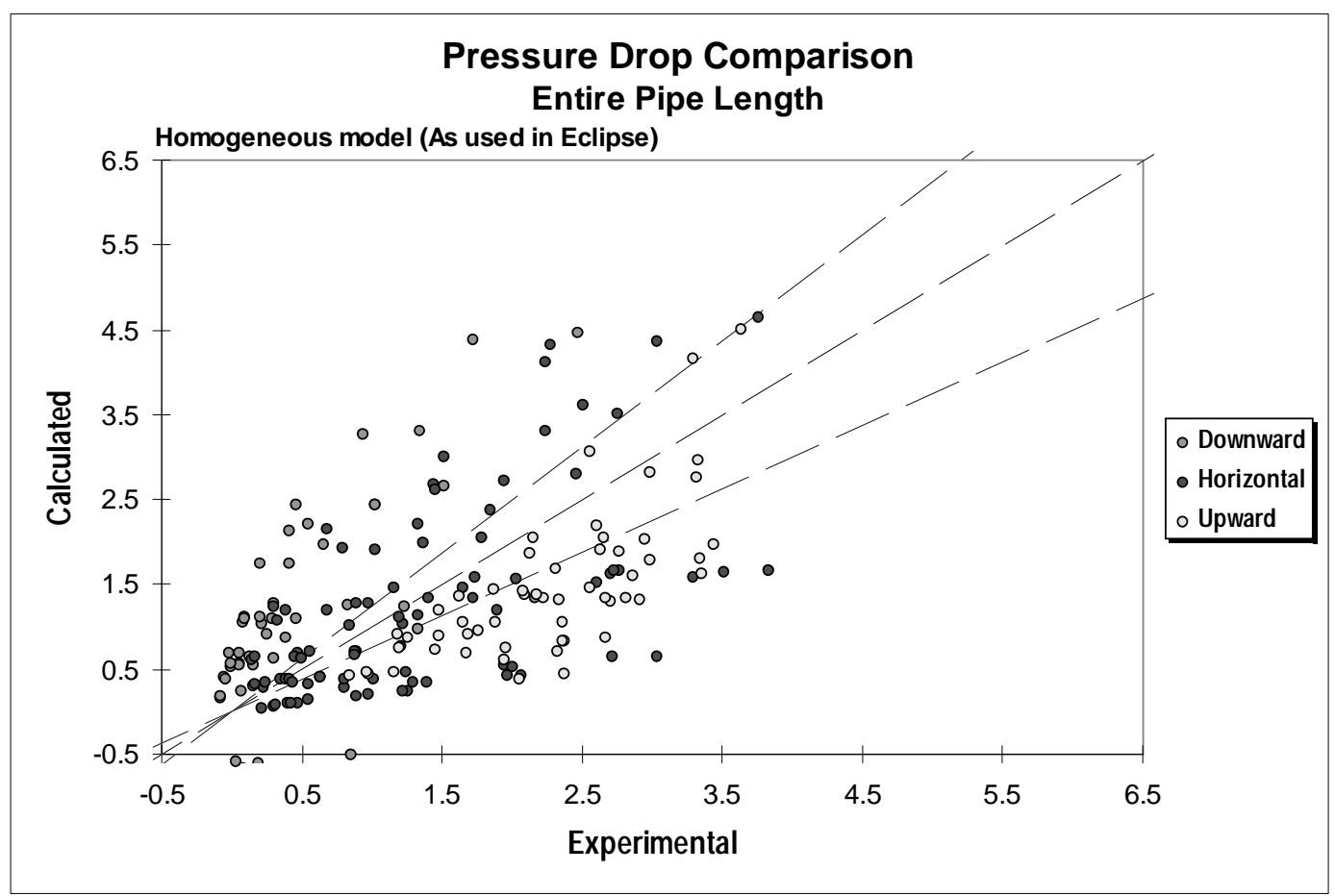

(b) Entire Pressure Drop

Figure 3.8 Comparison between Measured Pressure Drop and Prediction by the Homogeneous Model (1995 Experiments) 


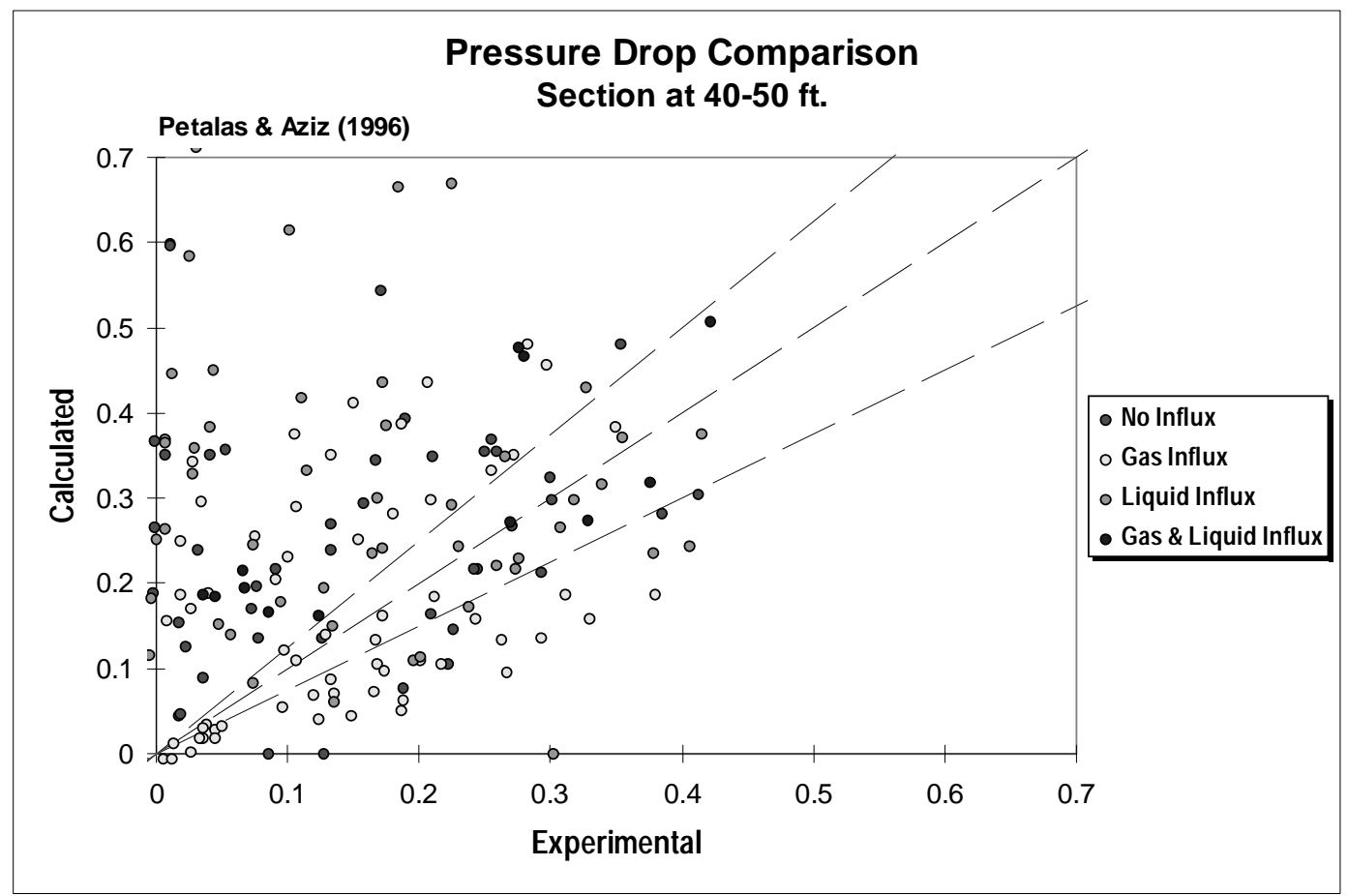

(a) Pressure Drop along the Section at 40-50 feet

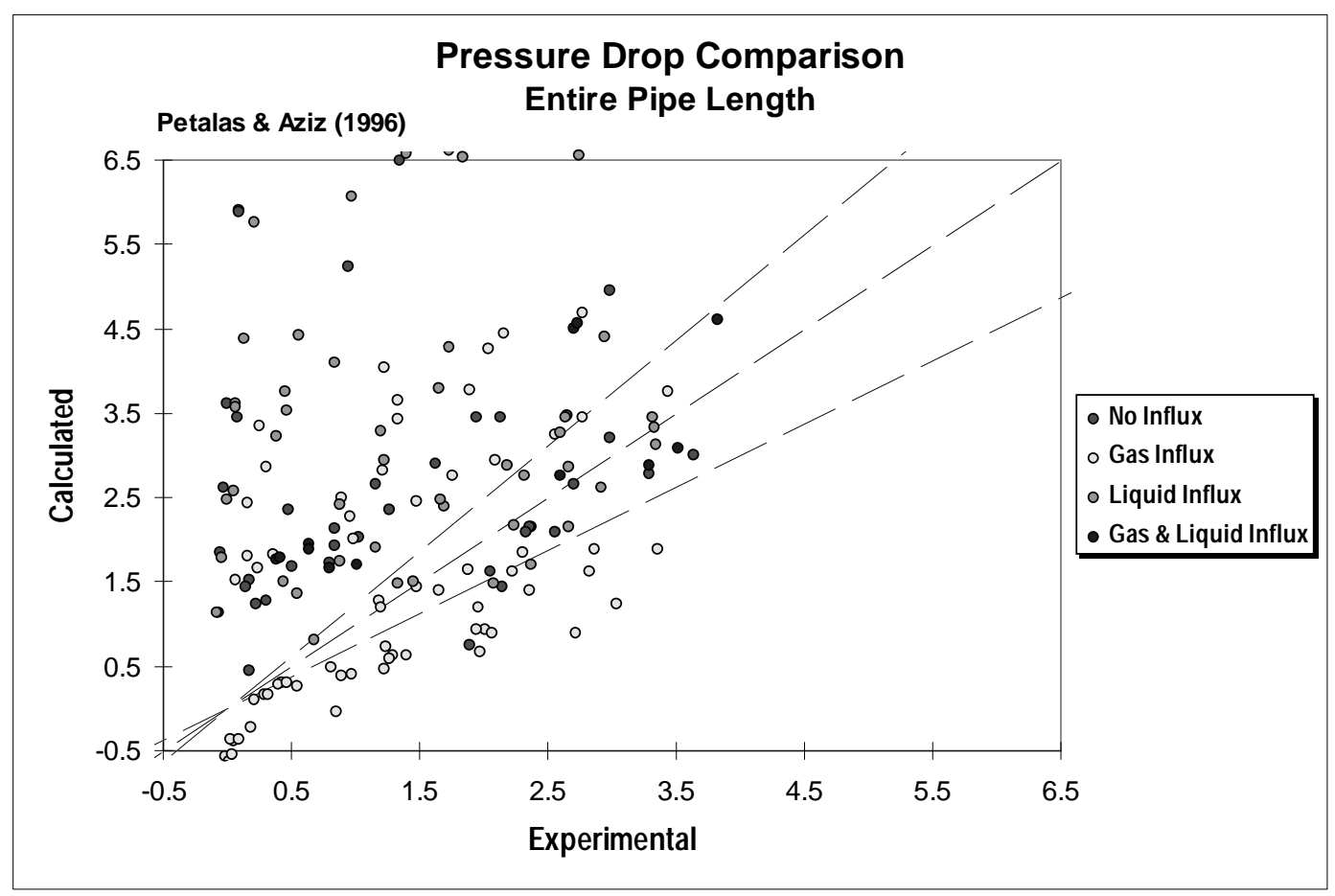

(b) Entire Pressure Drop

Figure 3.9 Comparison between Measured Pressure Drop and Prediction by Petalas \& Aziz (1996) Model (1995 Experiments) 


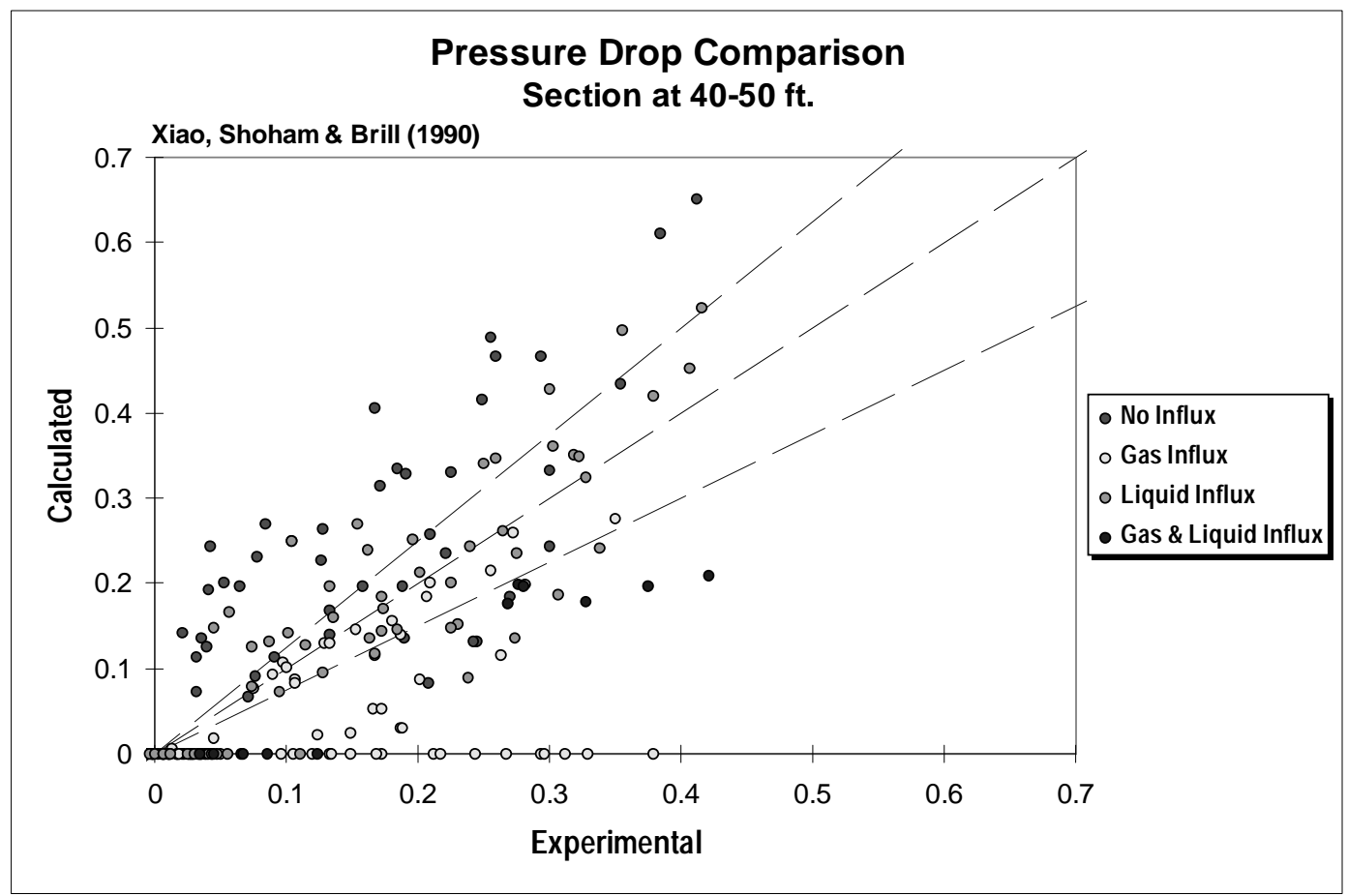

(a) Pressure Drop along the Section at 40-50 feet

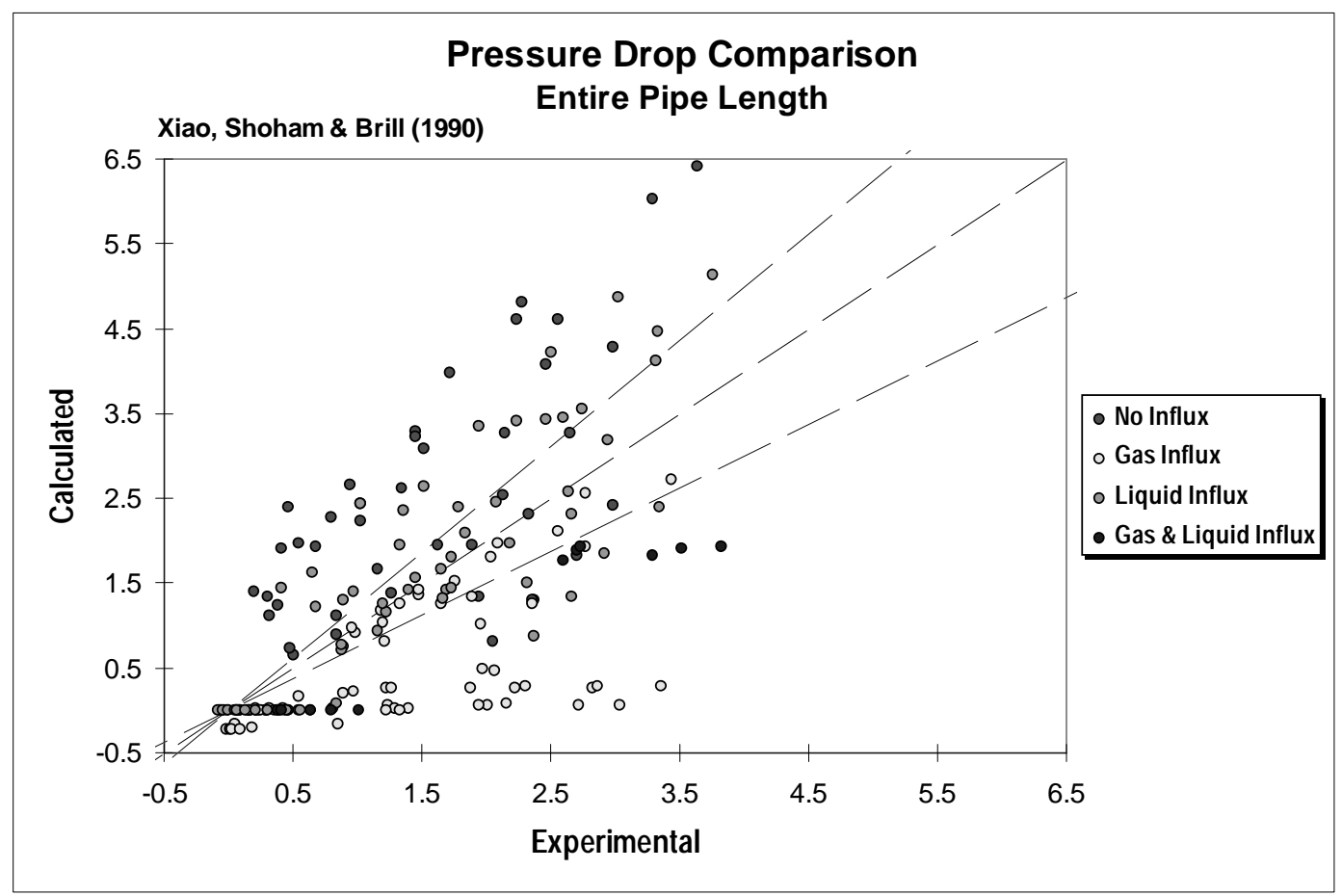

(b) Entire Pressure Drop

Figure 3.10 Comparison between Measured Pressure Drop and Prediction by Xiao, Shoham \& Brill (1990) Model (1995 Experiments) 


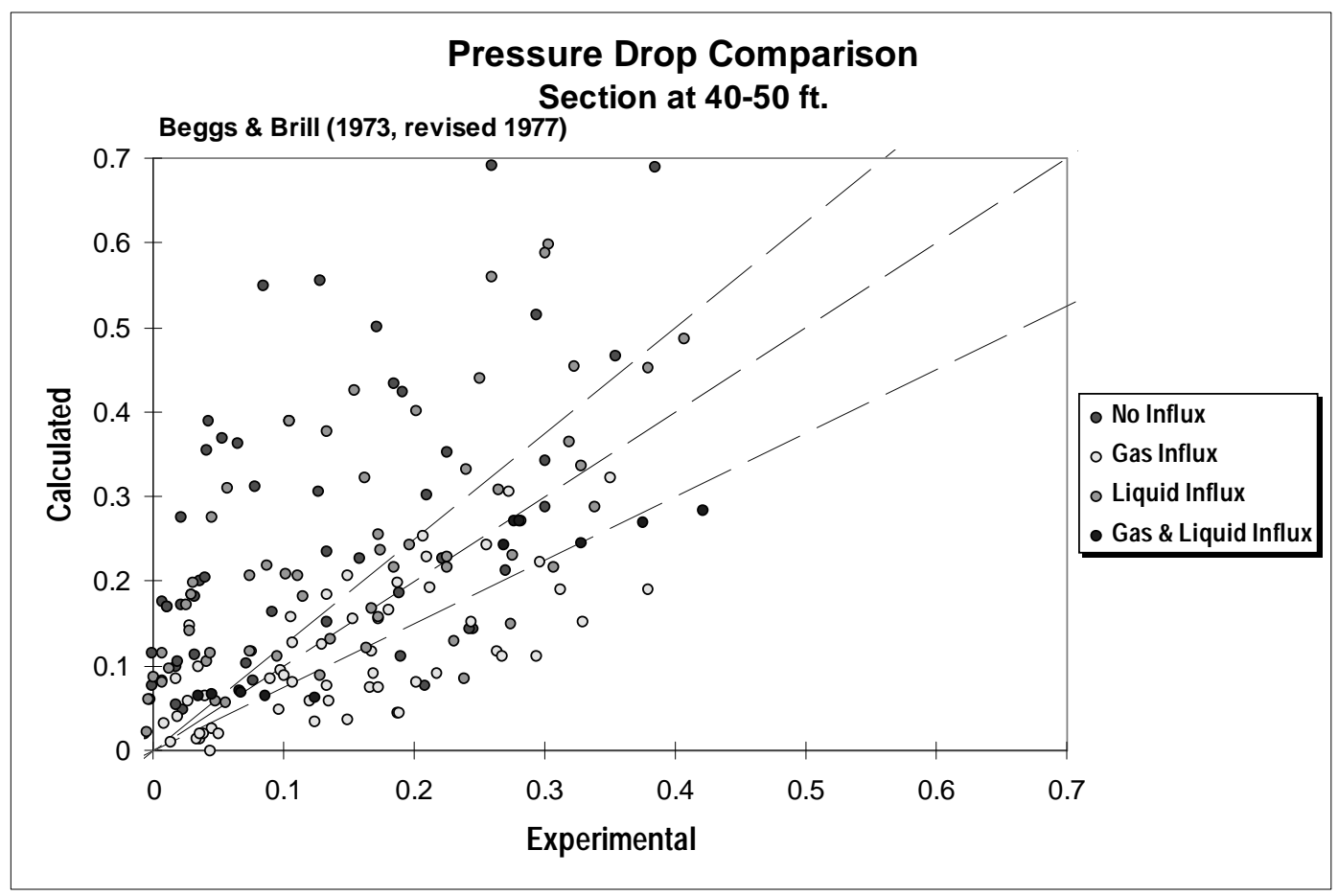

(a) Pressure Drop along the Section at 40-50 feet

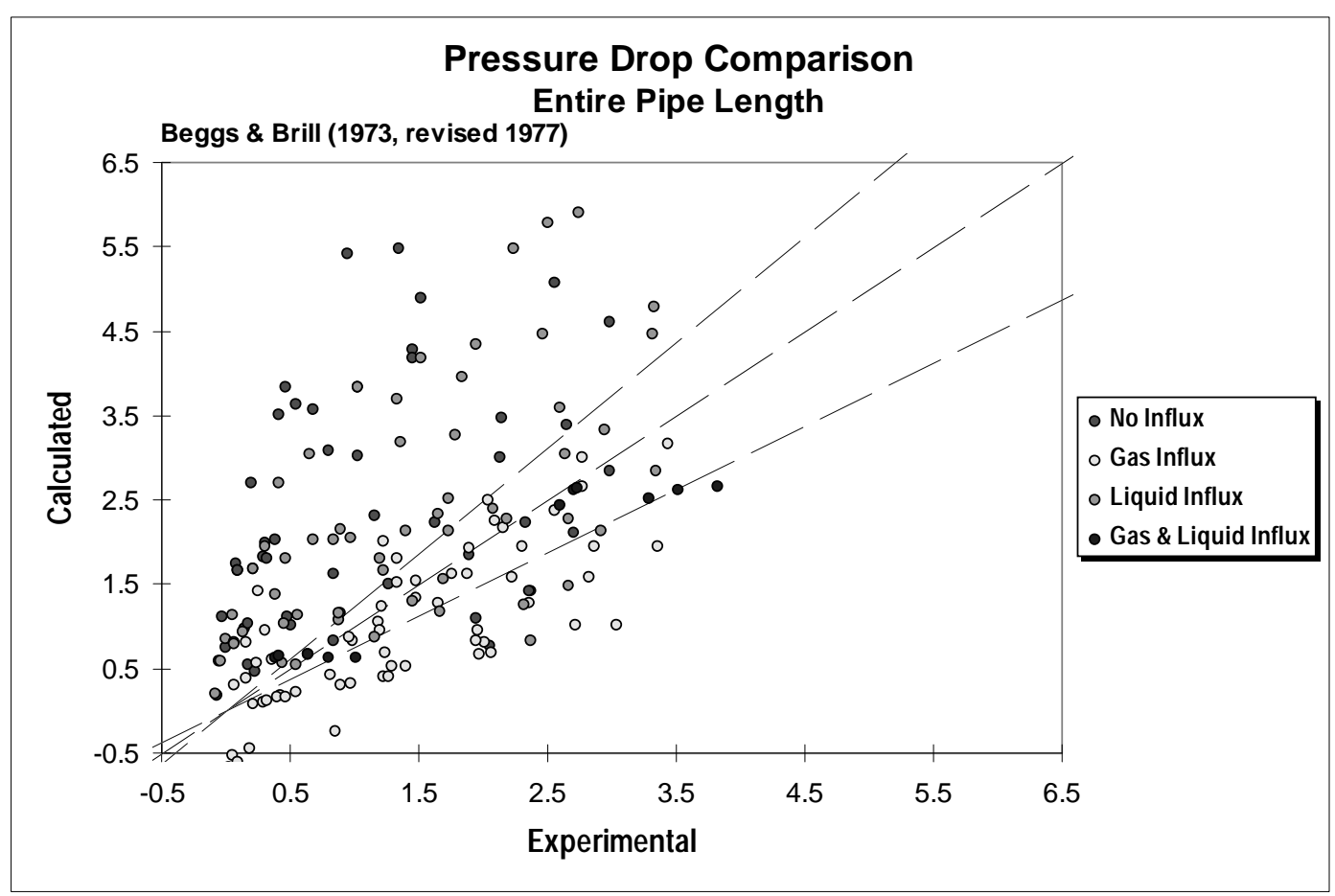

(b) Entire Pressure Drop

Figure 3.11 Comparison between Measured Pressure Drop and Prediction by Beggs \& Brill Model (1973, revised 1977) (1995 Experiments) 


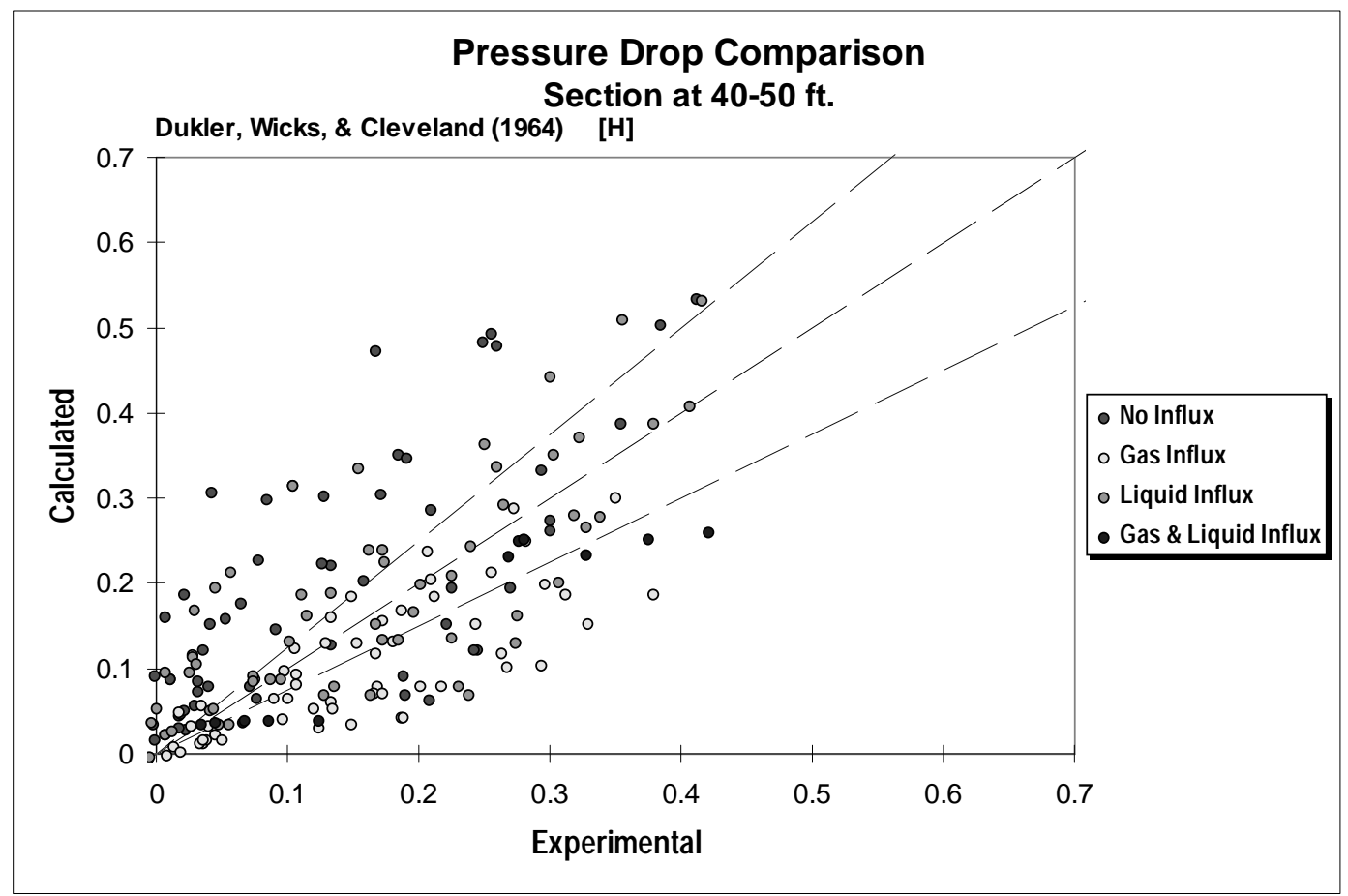

(a) Pressure Drop along the Section at 40-50 feet

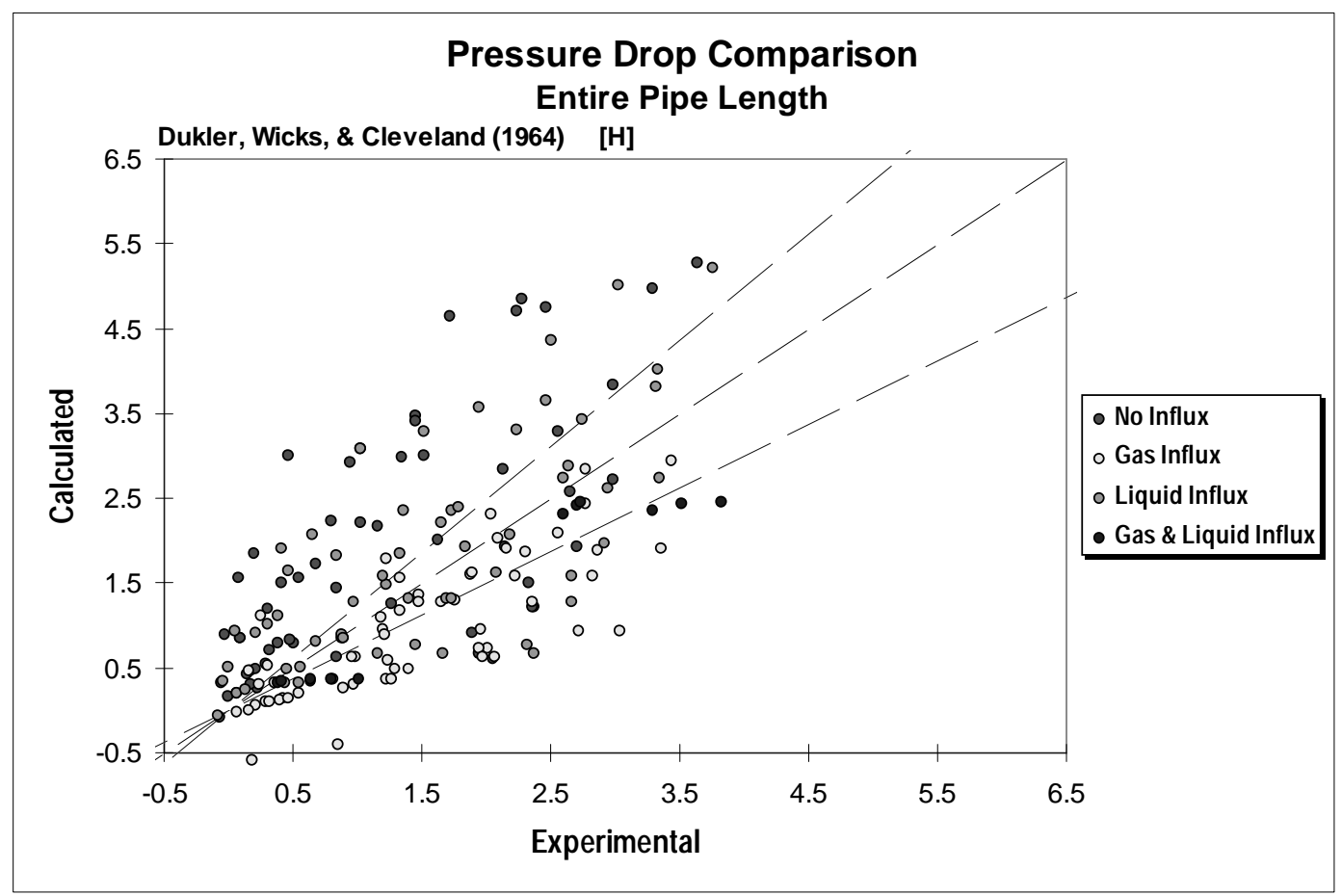

(b) Entire Pressure Drop

Figure 3.12 Comparison between Measured Pressure Drop and Prediction by Dukler, Wicks \& Cleveland (1964) Correlation (1995 Experiments) 


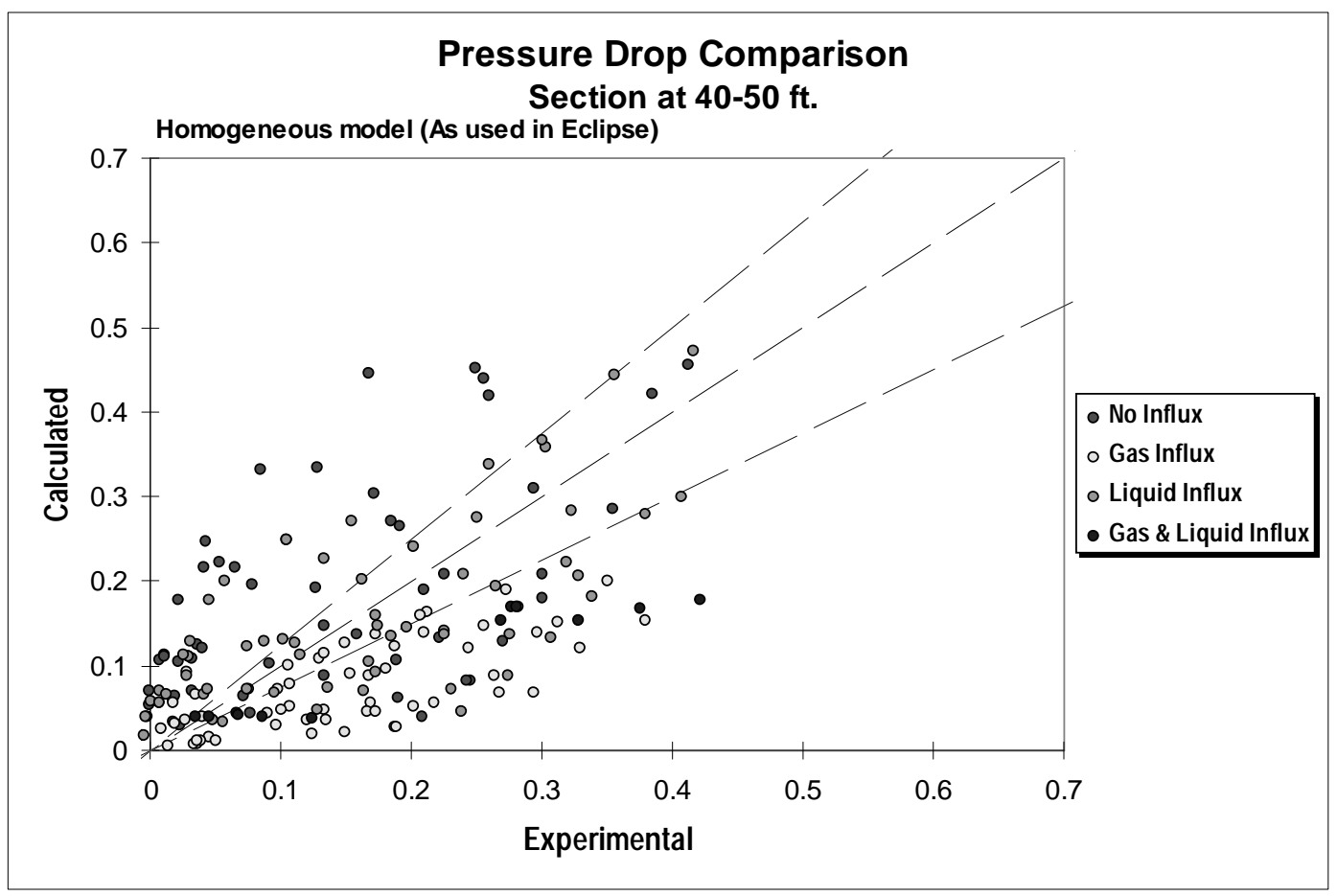

(a) Pressure Drop along the Section at 40-50 feet

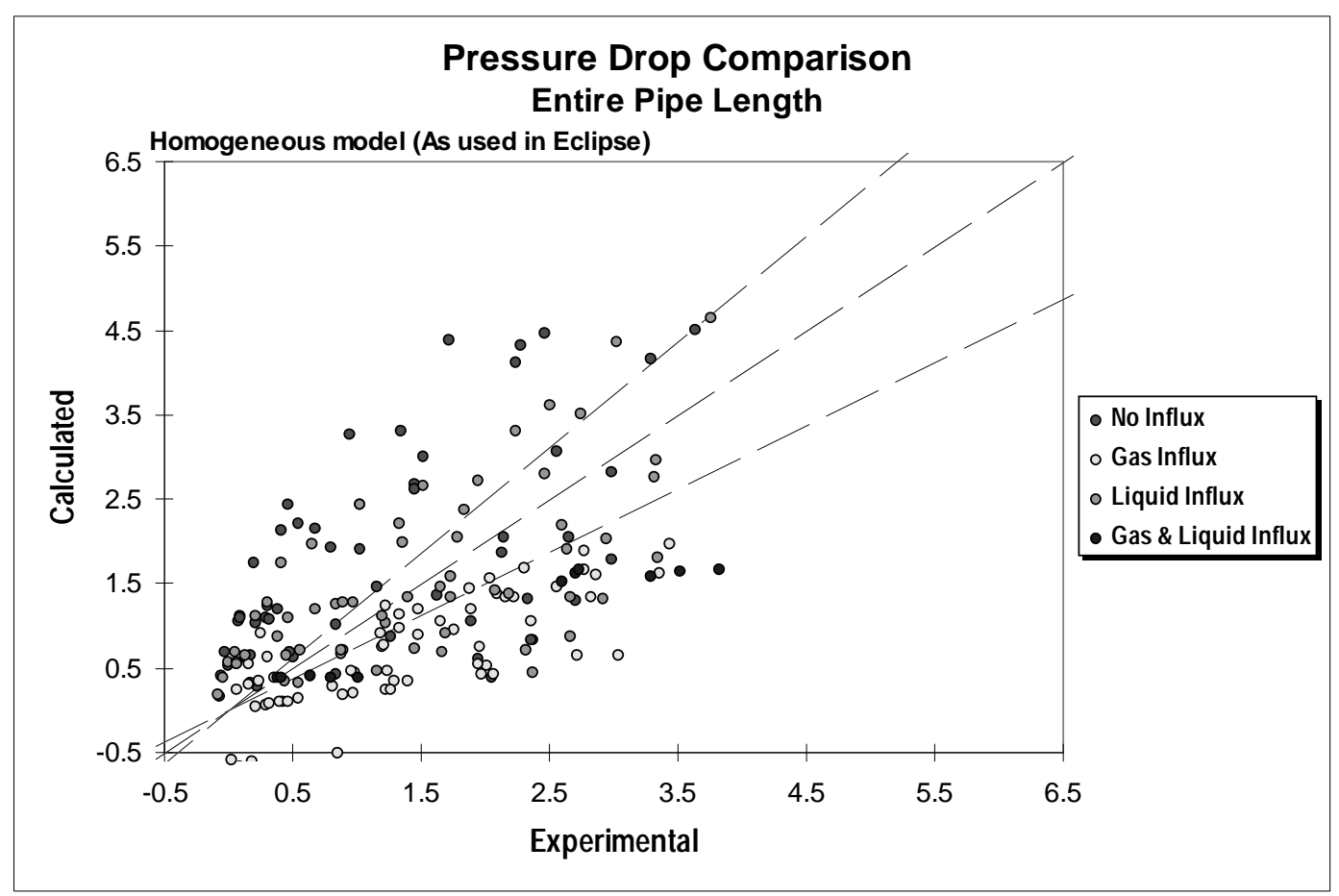

(b) Entire Pressure Drop

Figure 3.13 Comparison between Measured Pressure Drop and Prediction by the Homogeneous Model (1995 Experiments) 


\section{Pressure Drop Comparison \\ Entire Pipe Length}

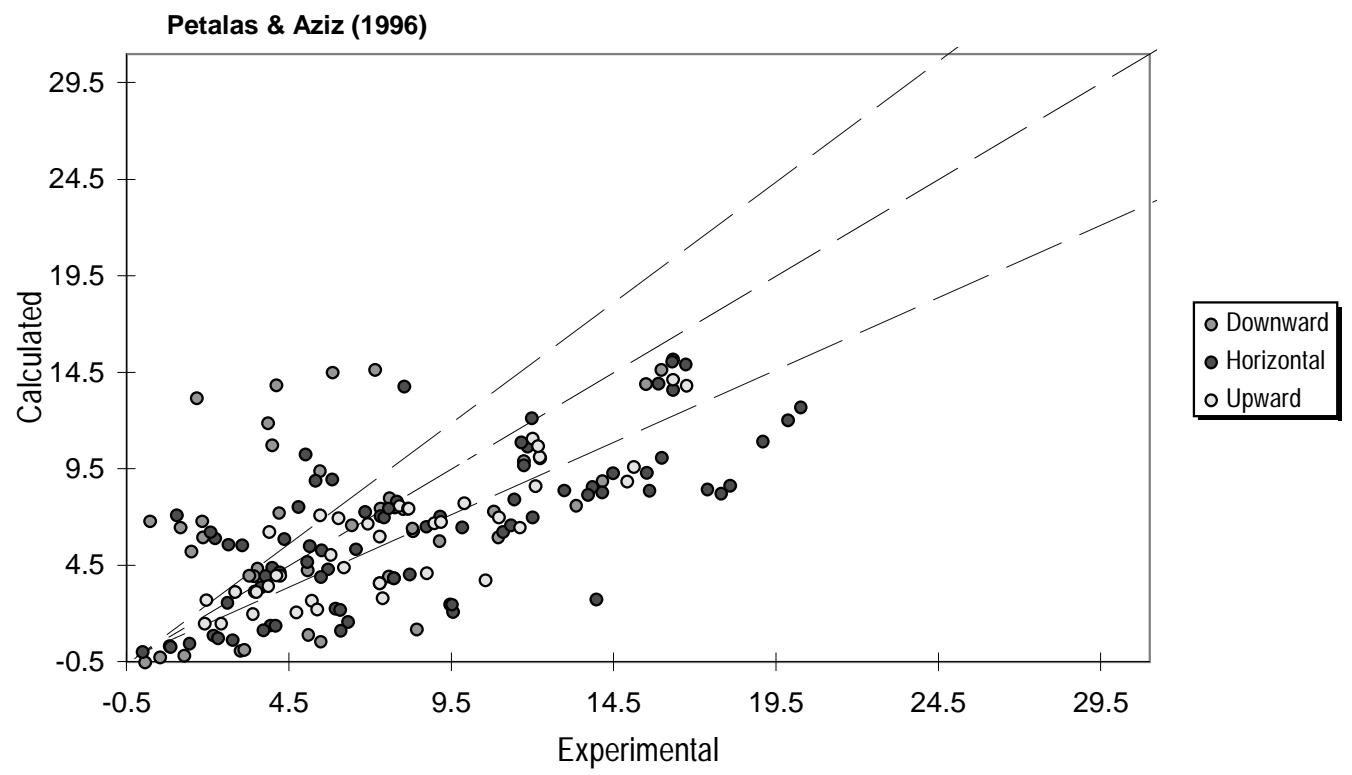

Figure 3.14 Comparison between Measured Pressure Drop and Prediction by Petalas \& Aziz (1996) Model (1996 Experiments)

\section{Pressure Drop Comparison Entire Pipe Length}

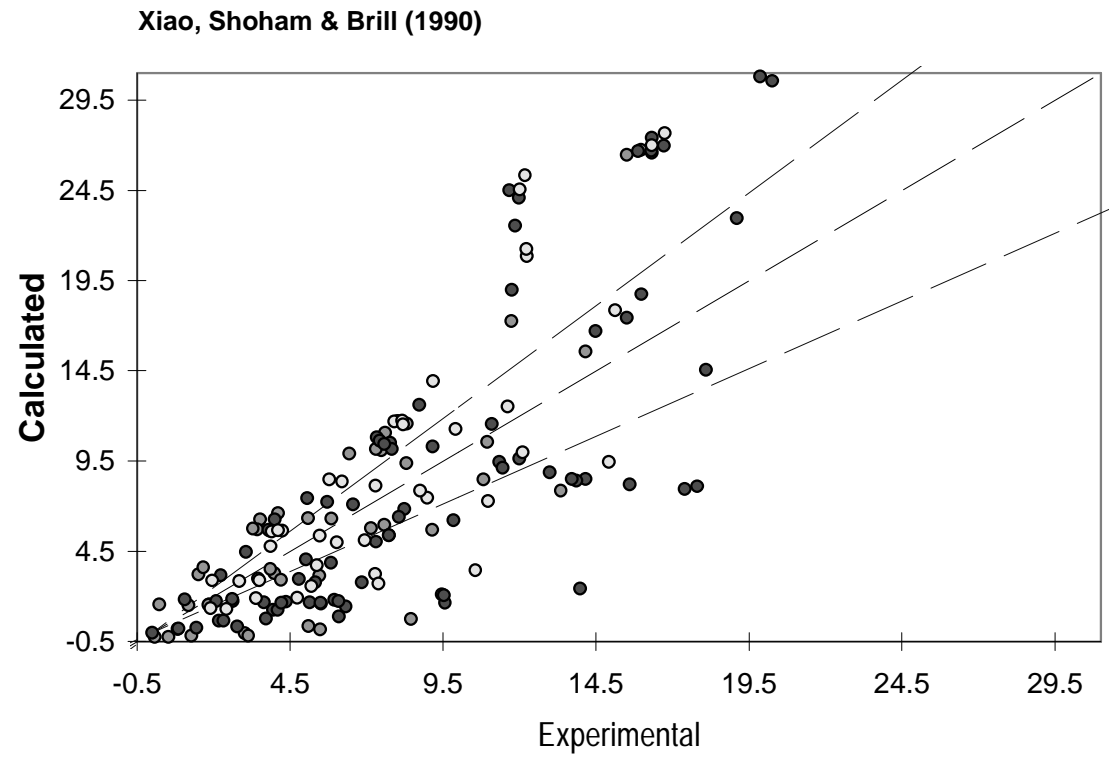

o Downward

- Horizontal

o Upward

Figure 3.15 Comparison between Measured Pressure Drop and Prediction by Xiao, Shoham \& Brill (1990) Model (1996 Experiments) 


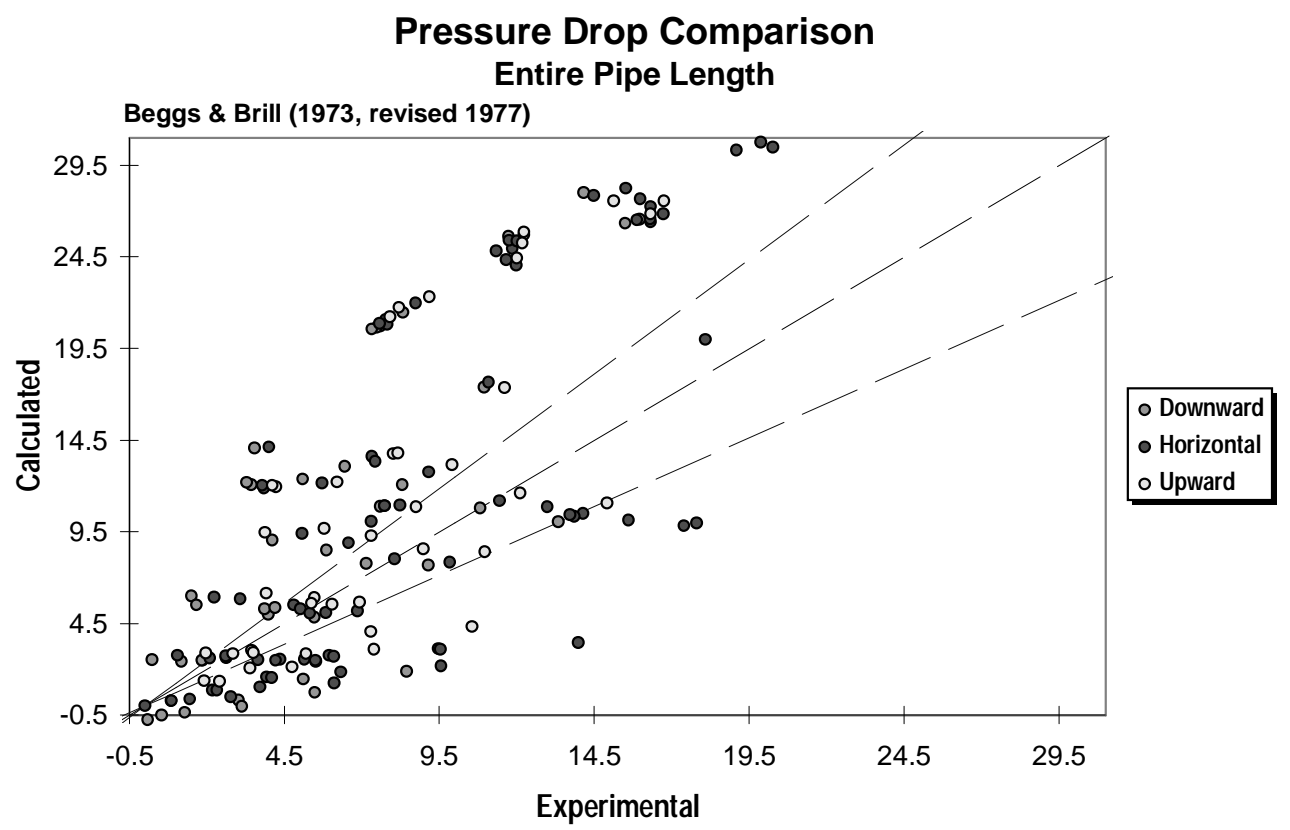

Figure 3.16 Comparison between Measured Pressure Drop and Prediction by Beggs \& Brill (1973, revised 1977) Model (1996 Experiments)

\section{Pressure Drop Comparison \\ Entire Pipe Length}

Dukler, Wicks, \& Cleveland (1964) $\quad[H]$

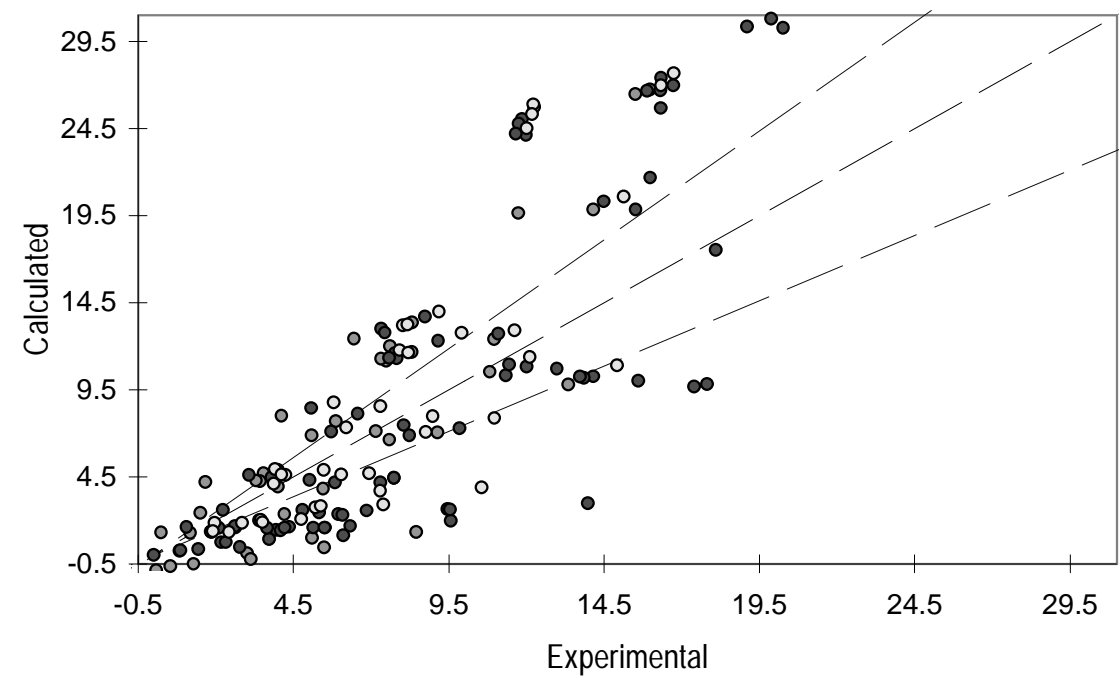

Figure 3.17 Comparison between Measured Pressure Drop and Prediction by Dukler, Wicks \& Cleveland (1964) correlation (1996 Experiments) 


\section{Pressure Drop Comparison \\ Entire Pipe Length}

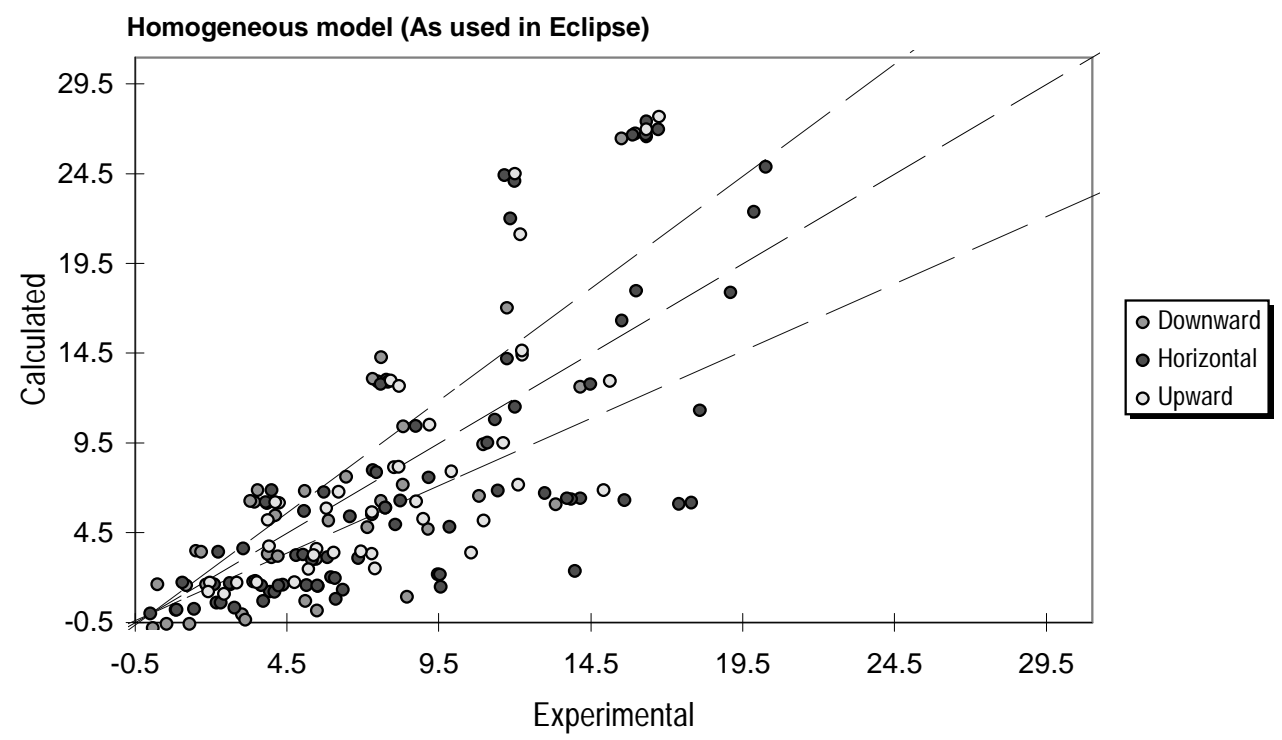

Figure 3.18 Comparison between Measured Pressure Drop and Prediction by the Homogeneous Model (1996 Experiments)

\section{Pressure Drop Comparison Entire Pipe Length}

Petalas \& Aziz (1996)

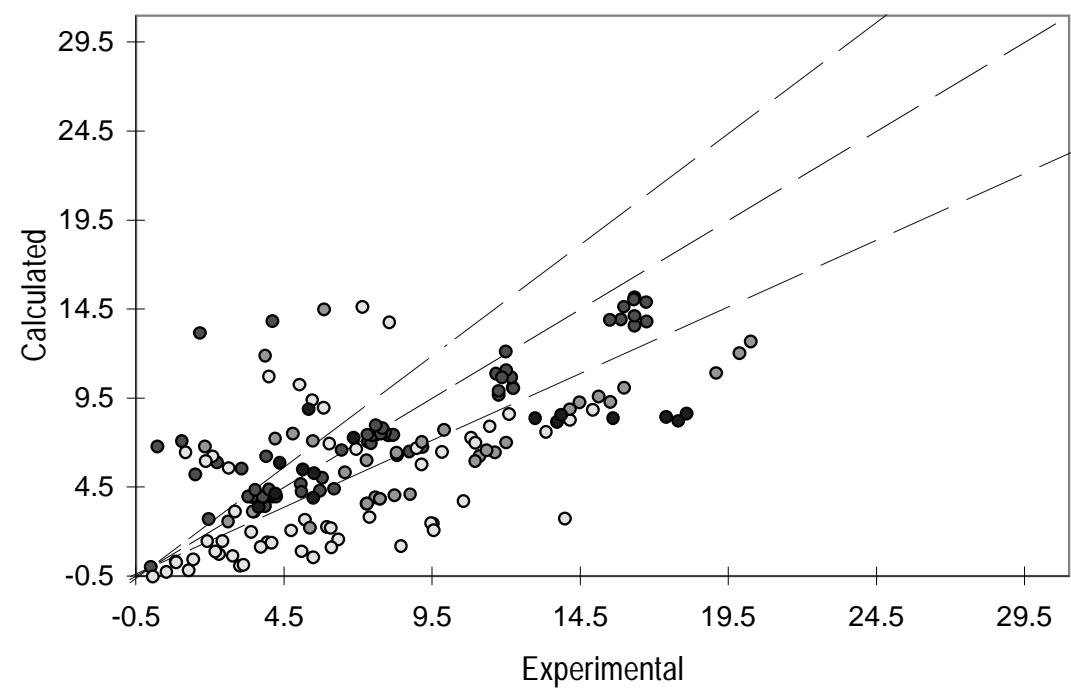

- No Influx

- Gas Influx

- Liquid Influx

- Gas \& Liquid Influx

Figure 3.19 Comparison between Measured Pressure Drop and Prediction by Petalas \& Aziz (1996) Model (1996 Experiments) 


\section{Pressure Drop Comparison \\ Entire Pipe Length}

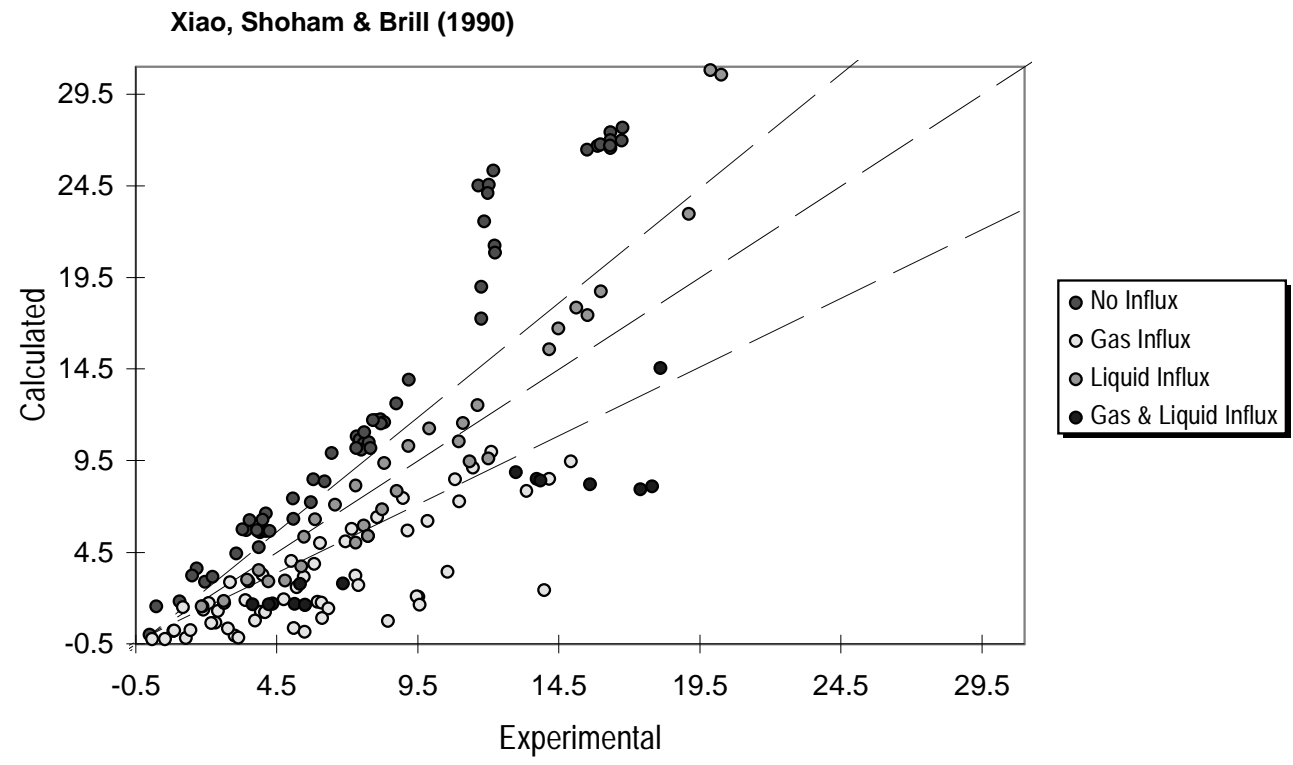

Figure 3.20 Comparison between Measured Pressure Drop and Prediction by Xiao, Shoham \& Brill (1990) Model (1996 Experiments)

\section{Pressure Drop Comparison}

Entire Pipe Length

Beggs \& Brill (1973, revised 1977)

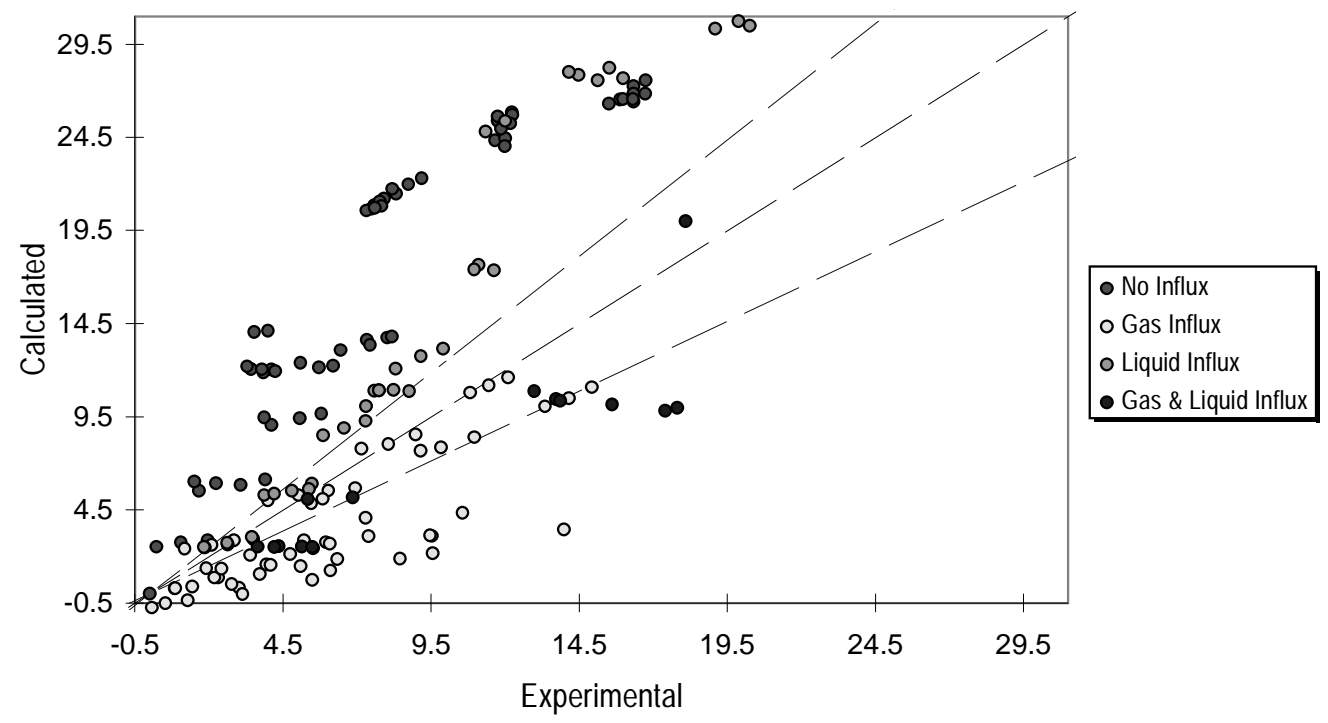

Figure 3.21 Comparison between Measured Pressure Drop and Prediction by Beggs \& Brill (1973, revised 1977) Model (1996 Experiments) 


\section{Pressure Drop Comparison \\ Entire Pipe Length}

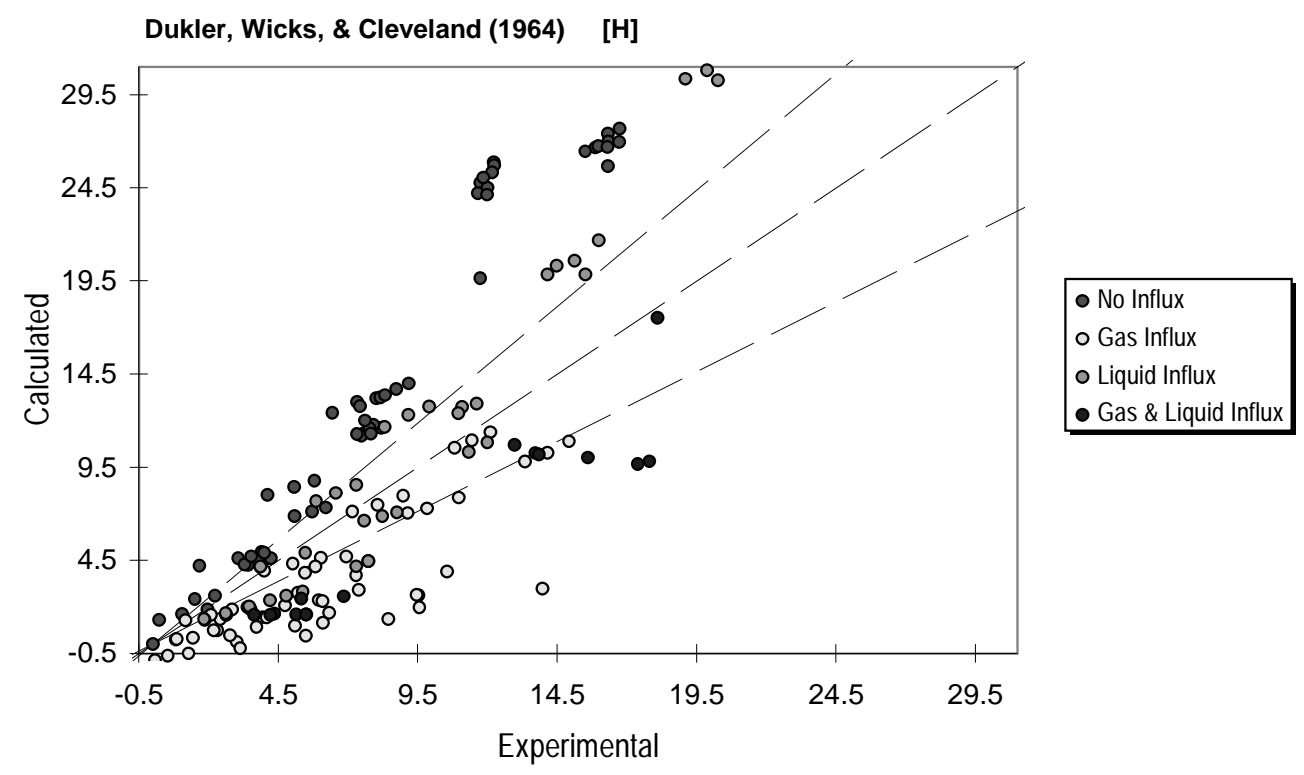

Figure 3.22 Comparison between Measured Pressure Drop and Prediction by Dukler, Wicks \& Cleveland (1964) Correlation (1996 Experiments)

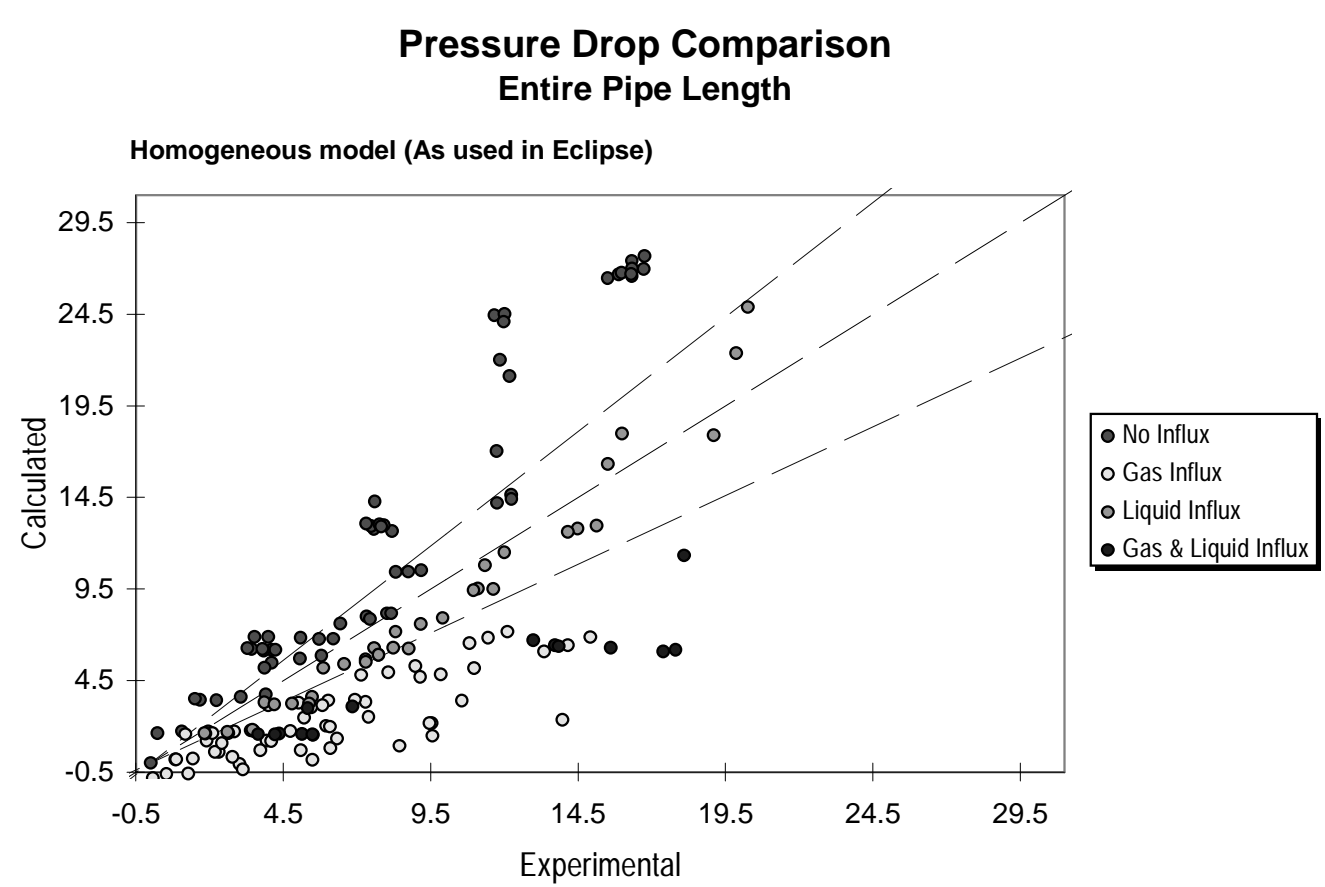

Figure 3.23 Comparison between Measured Pressure Drop and Prediction by the Homogeneous Model (1996 Experiments) 


\title{
4. Effects of Pressure Drop in Horizontal Wells and Optimum Well Length
}

\author{
by Raju Penmatcha, Sepehr Arbabi, and Khalid Aziz*
}

\begin{abstract}
As the length of a horizontal well is increased, its contact with the reservoir increases. But at the same time, the resistance to flow in the well also increases, which has a direct negative effect on the productivity of the well. The overall performance of a horizontal well depends on the balance of these two opposing factors. No reliable tools are currently available that account for both these factors in the evaluation of horizontal well performance. A semi-analytical wellmodel is developed which can quantify the effects of both single phase oil and two-phase oil/gas flow pressure loss in the well on the overall well performance. The model is quite flexible and can incorporate any friction factor correlation. A methodology is developed to show the effects of various reservoir, fluid and well parameters on well productivity. We demonstrate that ignoring frictional effects could lead to unrealistically high production estimates and low breakthrough times for water or gas. As a result of pressure drop in the well, breakthroughs occur first at the heel of the well. A methodology is also developed to calculate the optimum horizontal well length.
\end{abstract}

\subsection{Introduction}

Most of the analytical work done in the past ${ }^{1-16}$ on horizontal well productivity either assumed that the well is infinitely conductive or the flow is uniform along the entire well length. References 1-6 are based on the assumption of steady state flow in the reservoir, 7-9 are for pseudo steady-state flow conditions and 10-16 are for transient flow. The assumption of uniform flow was made purely for mathematical convenience. The infinite conductivity assumption is a good assumption only in certain cases when the pressure drop in the wellbore is very small compared to the drawdown in the reservoir, otherwise, the pressure drop in the wellbore should also be taken into account.

Previous work can be categorized into three types: (1) Models for wells that are infinitely conductive and thus are not influenced by pressure drop in the well, (2) models where the reservoir is represented by an analytical model for single phase flow, and (3) general models that couple multi-phase flow simulators with wells.

\footnotetext{
${ }^{*}$ This paper (SPE 37494) was presented at the 1997 SPE Production Operations Symposium held in Oklahoma City on March 9-11, 1997
} 


\subsubsection{Type1: Infinitely Conductive Well}

A horizontal wellbore can be considered to be infinitely conductive if the pressure drop in the wellbore is so small that ignoring it would not result in significant errors. Analytical equations with this assumption are available in the literature ${ }^{5,6,11-15}$. Based on the nature of the flow, any of the available equations can be used for quick performance evaluation of horizontal wells in single phase, homogeneous reservoirs. A numerical simulator, on the other hand, can be used for a thorough analysis of complex reservoirs. Using an analytical equation or a numerical simulator with the infinite conductivity well assumption for cases with significant pressure drop in the wellbore can lead to errors in the estimates. Most of the commercial simulators either ignore wellbore pressure drop or use inappropriate models to compute this term.

\subsubsection{Type2: Wellbore Coupled to an Analytically Approximated Reservoir}

Realizing the importance of wellbore pressure drop, a few groups have developed techniques to evaluate the effects of wellbore friction. This section outlines the available methods that couple the wellbore to an analytically approximated reservoir. The behavior of many homogenous, single phase reservoirs can be approximated analytically. Such analyses, though not too general in their applicability to complex reservoirs, give a good understanding of the nature of the problem. None of the approaches presented in this section take into account multiphase flow, or radial inflow effects in the wellbore.

Dikken ${ }^{17}$ was the first author to present an analytical model to couple turbulent flow in the horizontal wellbore to the flow in the reservoir. He showed that in most practical situations, a horizontal well will exhibit non-laminar (transition or turbulent) well flow. He considered steady state, single-phase pressure drop in the well due to laminar and turbulent flow in the wellbore and linked the wellbore to the reservoir using a material balance relationship. For turbulent flow, Blasius' formula was used to compute the friction factor. He solved this problem analytically for an infinite length horizontal well and numerically for a finite length horizontal well. Flow in the reservoir was defined using the PI (productivity index) relationship and he assumed that PI was constant along the well length.

Novy ${ }^{18}$ generalized Dikken's work by developing equations that could handle both single phase oil and single phase gas flow. In the case of gas flow, he also included the non-Darcy flow term in his analysis. He used a cylindrical reservoir drainage area, and stated that partial penetration or changing the shape of the drainage volume would not significantly alter his results. The problem was formulated as a boundary value problem and was solved using a finite difference scheme. He showed that for laminar flow in the wellbore, frictional effects reduce production rate by at least $10 \%$ when the wellbore pressure drop is more than $15 \%$ of the drawdown at the heel of the well. He also developed plots that can be used to determine combinations of well length and production rate that are immune to significant production loss due to frictional effects.

Ozkan et al. ${ }^{19}$ developed a model similar to that of Dikken, except they used a transient solution for the reservoir based on Green's function. Their model was presented in terms of dimensionless variables. 
Landman ${ }^{20}$ proposed further improvements to Dikken's model by allowing the PI to change along the wellbore. PI can vary along the well due to variation in perforation density, formation permeability or flow characteristics (radial/spherical flow). Using this model, he developed a methodology to calculate optimum perforation density along the well ( defined as the density that yields constant specific inflow along the well ). Forcing a constant specific inflow into the well delays breakthrough of gas or water.

\subsubsection{Type 3: Wellbore Coupled to a Numerically Approximated Reservoir}

In the papers discussed here, the wellbore was coupled to a numerical simulator. Since these works are not analytical it is harder to analyze the specific characteristics of the problem. But the advantage is that the numerical simulator can characterize a complex reservoir with multi-phase flow and hence it is more general.

Stone et al. ${ }^{21}$ applied energy, mass, and momentum balance equations both in the reservoir and the wellbore. The reservoir simulator was a time dependent, thermal, three phase, one dimensional model. In the wellbore, stratified, bubbly, slug and mist flow patterns were considered. Reservoir and the wellbore were coupled implicitly.

Islam et al. ${ }^{22}$ developed a model for radial influx into the wellbore based on experimental and numerical analyses. They developed mass and momentum balance equations for two-phase flow in the wellbore and coupled these to a numerical simulator. They suggested that bubbles created at the perforations due to radial influx and jet-like flow through the perforations induce additional turbulence in the wellbore.

Folefac et al. ${ }^{23}$ concluded that well length, well diameter and perforated interval have the most significant effect on the magnitude of pressure drop in the wellbore. They used a drift flux model in the wellbore and allowed for inter-phase mass transfer in the wellbore using an equation of state. They stated that production logging showed variation of flow rate along the well, which could be due to pressure drop in the wellbore.

Brekke et al. $^{24}$ developed a network wellbore simulator, called HOSIM, which can calculate pressure drop with up to three phases in the wellbore. Their approach is modular. However, the network model, representing the wellbore, has to be built on a PC before it can be coupled to the reservoir through the Eclipse simulator. For pressure drop in the well they provide a choice of three multi-phase flow models. The reservoir and the wellbore were connected using the PI approach. The PI was allowed to vary with time and location along the well length. They calculated the PI from the simulator runs by slightly perturbing the flow and then internally calculating $\mathrm{dQ} / \mathrm{dP}$ during every iteration.

None of the published works show how the magnitude of the effect of various reservoir, fluid and well variables on frictional pressure drop in the wellbore and thereby the well productivity. Here we present a methodology that can be used to assess the effect of various parameters on well productivity. Results are presented for the effects of well length, well flow rate, wellbore roughness, drawdown, fluid viscosity and reservoir permeability on well productivity. Many of the existing simulators neglect frictional pressure drop in the wellbore. Our work gives the reservoir engineer guidelines on determining when friction might be important. 
With pressure drop in the well, productivity does not continue to increase as the well length is increased. At some point the additional drilling and maintenance costs do not offset additional revenues. So far the literature does not provide a method of calculating the optimum length of horizontal wells. We present a method to calculate the optimum length of horizontal wells. The results can be expressed explicitly for the case of steady-state, single-phase flow in a homogeneous reservoir.

\subsection{Effect of Friction on Productivity}

Figure 4.1 shows a horizontal well in a reservoir. Let $p_{e}$ be the pressure at the outer boundary of the reservoir and $p_{w}(x)$ be the varying pressure along the wellbore due to frictional pressure drop. The well inflow equation can be written as

$q_{s}(x)=J_{s}(x)\left[p_{e}-p_{w}(x)\right]$

where $q_{s}(x)$ is the flow into the well per unit length of the wellbore and $J_{s}(x)$ is the productivity index per unit length of the wellbore. $J_{s}(x)$ can vary along the wellbore due to variation in perforation density, formation permeability or flow characteristics (spherical vs. radial flow).

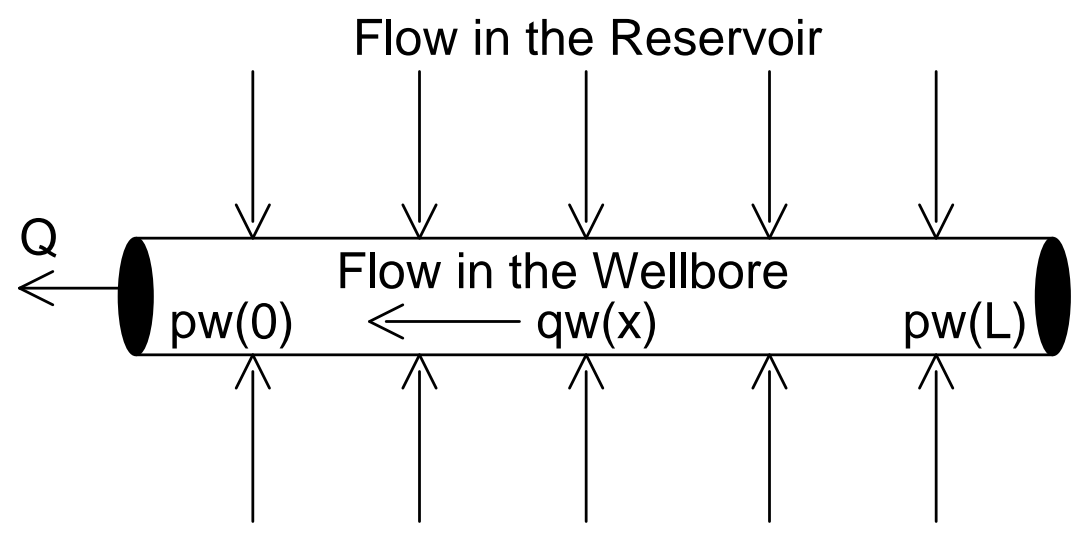

Figure 4.1: Reservoir/Wellbore Coupling

For simplicity, we assume that the reservoir is homogeneous and there is steady state, single phase oil flow in the system. The steady state flow assumption can be used in a reservoir where the oil production rate is equal to the water injection rate in a water flood operation. Since the fluid is single phase liquid, we assume that the density variations of the fluid along the 
wellbore are negligibly small. We also assume that the penetration ratio of the well in the drainage area is high enough so that we can practically neglect the end effects. This allows us to assume that $J_{s}(x)$ is constant along the wellbore. Using these assumptions, Eq.(4.1) can be integrated along the entire well to obtain the total flow rate from the well:

$$
Q_{w, f r i c}=\int_{0}^{L} q_{s}(x) d x=\int_{0}^{L} J_{s}\left[p_{e}-p_{w}(x)\right]=J_{s} p_{e} L-J_{s} \int_{0}^{L} p_{w}(x) d x
$$

If the pressure drop in the wellbore is neglected, then

$$
Q_{w, n o f}=\int_{x=0}^{L} q_{s}(x) d x=\int_{0}^{L} J_{s}\left[p_{e}-p_{w, 0}\right] d x=J_{s} L\left[p_{e}-p_{w, 0}\right]
$$

where $p_{w, 0}$ is the pressure at the heel of the well. We define a variable called Productivity Error, P.E., which gives us the error in the well productivity calculations as a result of neglecting frictional pressure drop in the wellbore. P.E. is defined as:

$$
\text { P.E. }=\frac{Q_{w, n o f}-Q_{w, f r i c}}{Q_{w, n o f}}
$$

Substituting Eq.(4.2) and Eq.(4.3) in Eq.(4.4), we get

$$
\text { P.E. }=\frac{J_{s} L\left(p_{e}-p_{w, 0}\right)-J_{s} p_{e} L+J_{s} \int_{0}^{L} p_{w}(x) d x}{J_{s} L\left(p_{e}-p_{w, 0}\right)}=\frac{\frac{1}{L} \int_{0}^{L} p_{w}(x) d x-p_{w, 0}}{\left(p_{e}-p_{w, 0}\right)}=\frac{\frac{1}{L} \int_{0}^{L}\left[p_{w}(x)-p_{w, 0}\right] d x}{\left(p_{e}-p_{w, 0}\right)}
$$

Hence,

$$
\text { P.E. }=\frac{\text { average wellbore pressure drop }}{\text { drawdown at the heel }}
$$

Eq.(4.6) indicates that pressure drop in the wellbore becomes significant only if it is comparable to the drawdown at the heel of the well. We will use this equation in the sensitivity analysis section later where we will show the effect of various parameters on well productivity.

\subsection{Flow Rate vs. Well Length}

Mass balance gives the following equation for flow in the wellbore: 


$$
\frac{d}{d x}\left[q_{w}(x)\right]=-q_{s}(x)
$$

where $q_{w}(x)$ is the flow rate in the wellbore and it varies along the length of the wellbore. After some mathematical manipulation (see Appendix A), we can obtain the following relationship to calculate the required well length after the drawdown at the heel and the required well flow rate are specified.

$$
L=\int_{0}^{Q} \frac{d q_{w}(x)}{\sqrt{2 J_{s} \int_{Q}^{q_{w}}\left[\frac{d p_{w}}{d x}\right] d q_{w}(x)+\left[J_{s} \Delta p_{0}\right]^{2}}}
$$

In the above equation, $\frac{d p_{w}}{d x}$ is the pressure gradient in the wellbore, $L$ is the length of the well, and $\Delta p_{0}$ is the drawdown at the heel of the well. Equation (4.8) is independent of the method of calculating wellbore pressure drop. This gives us the flexibility of using any single phase or multi-phase correlation to obtain the pressure drop in the wellbore. In this study, the pressure drop in the well due to friction is calculated using the following equations:

$$
\frac{d p_{w}(x)}{d x}=1.079 \times 10^{-4} f\left[\frac{\rho U^{2}}{D}\right]=7.294 \times 10^{-12} f\left[\frac{q_{w}^{2} \rho}{\pi^{2} D^{5}}\right]
$$

where $f$ is Moody's friction factor. The flow is considered laminar for $N_{\mathrm{Re}}<2100$, where $N_{\mathrm{Re}}$ is the Reynolds number. For $N_{\mathrm{Re}} \geq 2100$, turbulent flow equations are used.

Reynolds number is calculated as,

$$
N_{\mathrm{Re}}=0.1231 \frac{q_{w} \rho}{\mu D}
$$

For laminar flow,

$$
f=\frac{64}{N_{\mathrm{Re}}}
$$

is used, and for turbulent flow Jain's approximation ${ }^{25}$ of Colebrook-White equation is used. Typically, the flow rate in the well starts out to be laminar at the toe and then as the flow in the well increases towards the heel it slowly becomes turbulent. An equation that accounts for radial influx into the wellbore, for example, can be easily used in this model, as long as the derivative can be expressed as a function of the variable of integration in Eq.(4.8), $q_{w}$. 


\subsection{Sensitivity Analysis}

Using the parameters in Table 4.1 and the equations developed so far, the effects of friction on well productivity are illustrated below. All the cases described below are based on steady state flow in the reservoir with single phase oil in the wellbore. The steady-state equations developed by Economides et al. ${ }^{6}$ are used for describing the flow in the reservoir. The productivity index per unit length $\left(J_{s}\right)$ is obtained by dividing the productivity index of the horizontal well with its length. $J_{s}$ is then assumed constant along the entire horizontal well length.

Table 4.1: Parameters Used in Example Problem

$\begin{array}{lc}\text { Horizontal Permeability } & 3000 \mathrm{mD} \\ \text { Vertical Permeability } & 300 \mathrm{mD} \\ \text { Reservoir Thickness } & 50 \mathrm{ft} \\ \text { Outer Boundary Pressure } & 5000 \mathrm{psia} \\ \text { Oil FVF } & 1.18 \\ \text { Gas FVF } & 0.0045 \\ \text { Oil Viscosity } & 1.0 \mathrm{cP} \\ \text { Gas Viscosity } & 0.02 \mathrm{cP} \\ \text { Oil Density } & 40.0 \mathrm{lbm} / \mathrm{cu} . \mathrm{ft} \\ \text { Gas Density } & 2.0 \mathrm{e}-04 \mathrm{lbm} / \mathrm{cu} . \mathrm{ft} \\ \text { Drainage Area } & 650 \mathrm{acres} \\ \text { Well Radius } & 3.0 \mathrm{inch} \\ \text { Skin } & 0.0 \\ \text { Wellbore Roughness } & 0.0 \mathrm{ft}\end{array}$

\subsubsection{Effect of Well Length}

Figure 4.2 shows the effect of flow rate and well length on well friction. The solid lines in the plot show the variation of oil production rate with well length for three different drawdowns. The drawdowns are low because of the high permeability of the reservoir. The concave shape of these lines shows the decrease in incremental production with well length. For example, for a 5 psi drawdown the flow rates of $8000 \mathrm{STB} /$ day can never be reached with any well length. If friction were neglected in the wellbore, then the well productivity continues to increase with well length. 
This figure also shows lines of constant P.E.. For a given drawdown at the heel of the well (denominator in the RHS of Eq.(4.6) held constant), as the well length increases, pressure drop in the wellbore increases due to the increase in the flow rate. Hence, the longer the well, the larger is the ratio of pressure drops for a given drawdown, and hence the higher the P.E. from Eq.(4.6). As expected, frictional effects become more important as the horizontal well length increases.

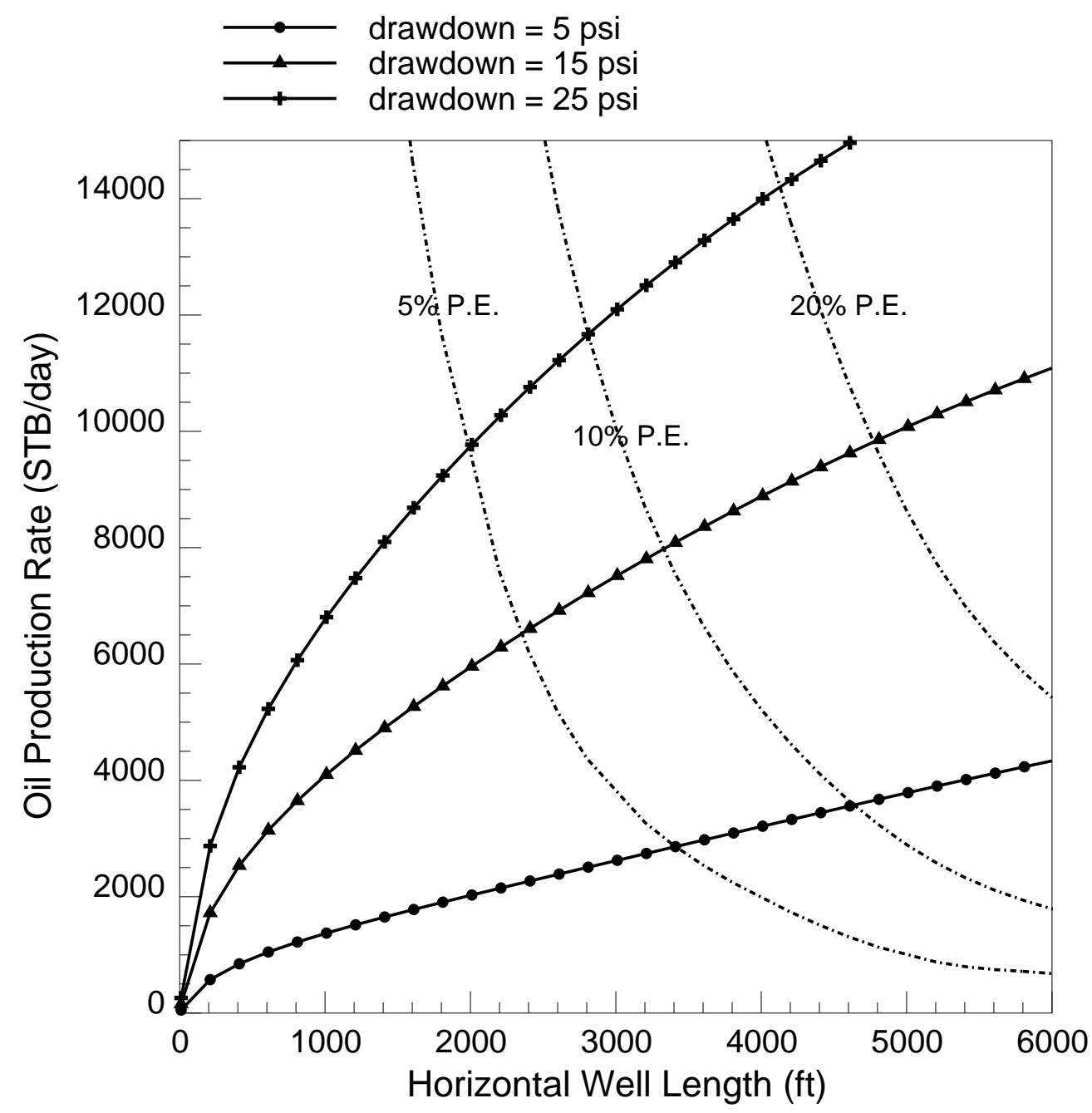

Figure 4.2: Effect of Flow Rate and Well Length on Wellbore Friction

\subsubsection{Effect of Flow Rate}

As discussed, the pressure drop in the wellbore can increase faster than that in the reservoir. The flow rate from a given horizontal well in a reservoir can be altered by changing the drawdown. Keeping all parameters constant except the flow rate from the well and the drawdown at the heel of the well we can show some interesting results. 
Assuming for flow in the reservoir to be laminar, from Eq.(4.3),

$\Delta p_{0} \propto Q_{n o f}$

Blasius' formula shows that for a smooth pipe with turbulent flow,

$f=C N_{\mathrm{Re}}^{-0.25}$

Combining Eq.(4.13) with Eqs.(9) and (10), we can show that

$\Delta p_{\text {turbulent }} \propto q_{w}^{1.75}$

Now consider the case when the drawdown at the heel is increased by lowering the pressure at the heel of the well, $p_{w, 0}$. Based on Eq.(4.12), $Q_{n o f}$ increases linearly due to an increase in drawdown. Let us consider the increase in $Q_{\text {fric }}$ due to the increase in drawdown. The increase in drawdown increases the flow rate $q_{w}(x)$ along the well. And because of turbulent flow in the wellbore pressure drop in the wellbore increases at a higher rate than the drawdown at the heel. Following Eq.(4.2), this causes $Q_{\text {fric }}$ to increase at a sub-linear rate with an increase in the drawdown at the heel. Hence, using Eq.(4.4), an increase in drawdown at the heel increases the numerator of Eq.(4.4) super-linearly while the denominator is increasing linearly. This causes the P.E. to increase with an increase in drawdown. Since an increase in drawdown increases the flow rate from the well we can also say that an increase in the well flow rate causes the P.E. to increase and vice-versa.

This result can be illustrated using Figure 4.2. Let us assume that the well length is fixed at $3000 \mathrm{ft}$. The flow rate from this well can be increased by increasing the drawdown. When the drawdown is 5 psi, the flow rate is about $2600 \mathrm{STB} /$ day and the P.E. is below 5\%. By increasing the drawdown to $15 \mathrm{psi}$, the flow rate can be increased to $7500 \mathrm{STB} /$ day. But the P.E. has also increased and it now lies between 5\% and 10\%. The P.E. goes above $10 \%$ when the drawdown is increased to $25 \mathrm{psi}$. So for a fixed well length, the higher the flow rate, the more important the frictional pressure drop in the wellbore. This suggests that frictional effects will be higher for a well during its early life when the flow rate is the highest.

\subsubsection{Effect of Wellbore Roughness}

The effect of wellbore roughness is shown in Figure 4.3. The dashed lines in this figure are calculated using the parameters in Table 4.1. The solid lines are drawn for the case when the wellbore relative roughness is increased from 0.0 to 0.1 (such a high value of effective roughness may be caused by open hole completion or due to fluid influx into the well) and keeping everything else the same as in Table 4.1. Drawdown is 25 psi.

For a well of length $3000 \mathrm{ft}$, increasing the wellbore roughness does two things. It decreases the production rate and increases the P.E.. The increase in P.E. in this case is from approximately $11 \%$ to $26 \%$. It can also be observed that for a fixed production rate, the required well length increases with the increase of wellbore friction. 


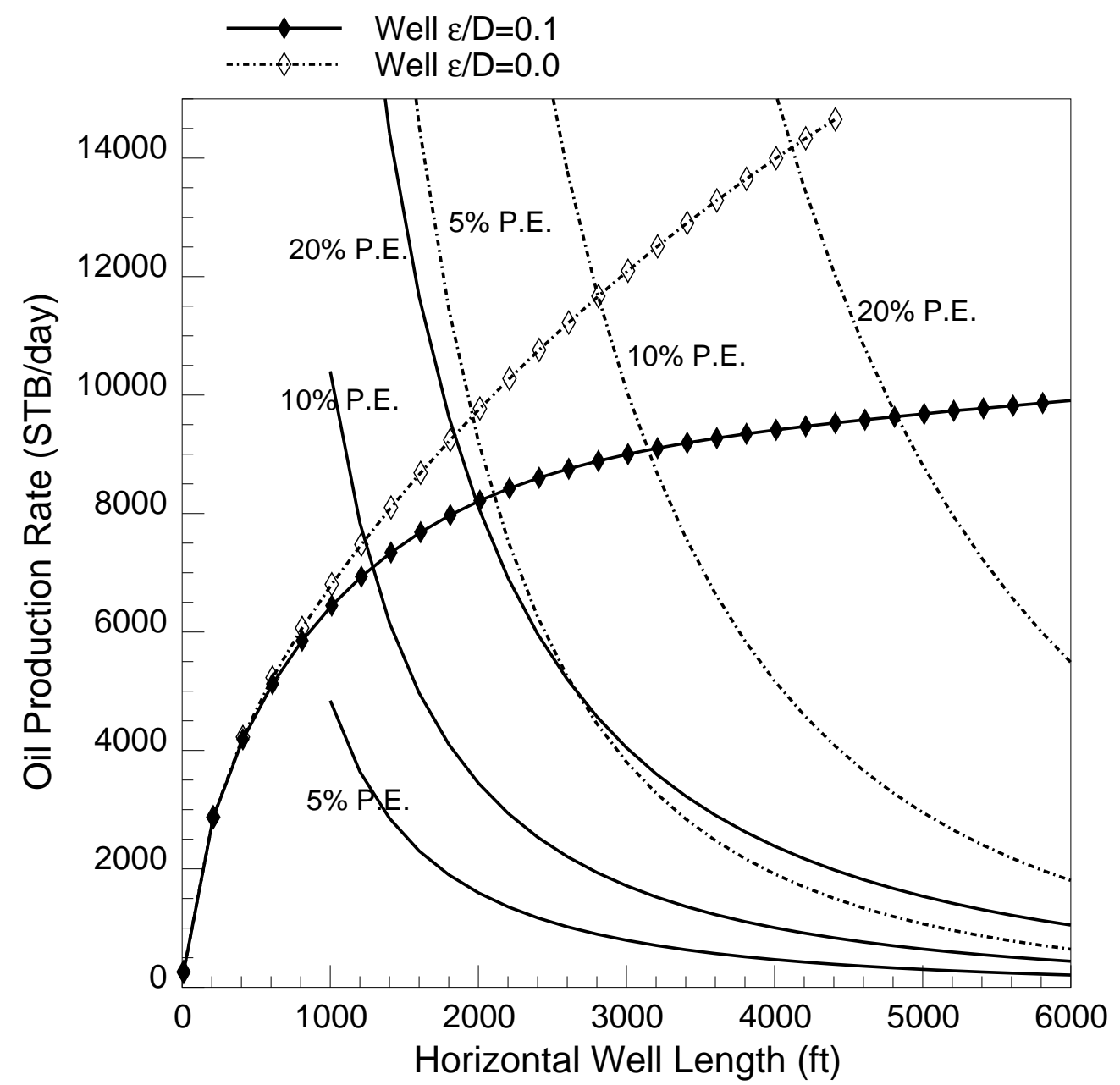

Figure 4.3: Effect of Well Roughness on Wellbore Friction $($ Drawdown=25 psi)

\subsubsection{Effect of Fluid Viscosity}

The flow in the reservoir is almost always laminar, while in the wellbore the flow can be turbulent. Because of this, an increase in fluid viscosity causes the pressure drop in the wellbore to increase less than the increase in the reservoir. This gives the interesting result that the P.E. can be lower for higher viscosity fluids. This effect is illustrated in Figure 4.4. The dashed lines are drawn using the parameters in Table 4.1 with 15 psi drawdown. The bold lines are drawn by increasing the viscosity in Table 4.1 to $10 \mathrm{cP}$ and drawdown to 150 psi. As shown in Figure 4.4, for producing $8000 \mathrm{STB} / \mathrm{day}$, the required horizontal well lengths for the two cases shown are not very different. But the P.E. for the case with $10 \mathrm{cP}$ viscosity is less than $2 \%$ while that for the case with $1 \mathrm{cP}$ is much higher at $10 \%$. 


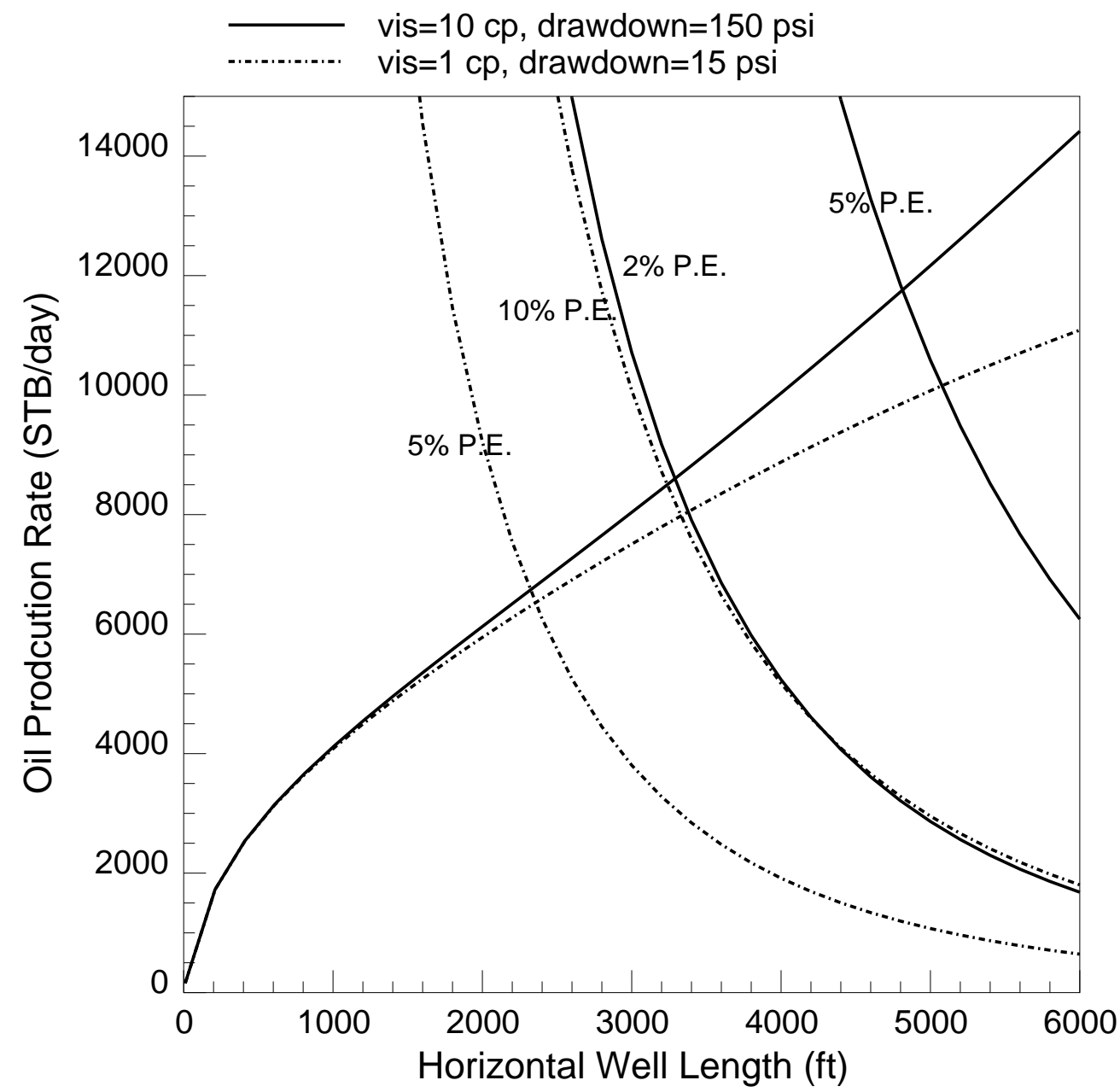

Figure 4.4: Effect of Oil Viscosity on Wellbore Friction

\subsubsection{Effect of Reservoir Permeability}

The effect of reservoir permeability is shown in Figure 4.5. The dashed lines are drawn using the parameters in Table 4.1 for single phase oil flow with 15 psi drawdown. The bold lines are drawn by decreasing the horizontal permeability from $3000 \mathrm{mD}$ to $300 \mathrm{mD}$ and increasing the drawdown to $150 \mathrm{psi}$. The vertical to horizontal permeability ratio was kept constant at 0.1 in both these cases. This figure clearly indicates that the P.E. increases with increasing reservoir permeability. 


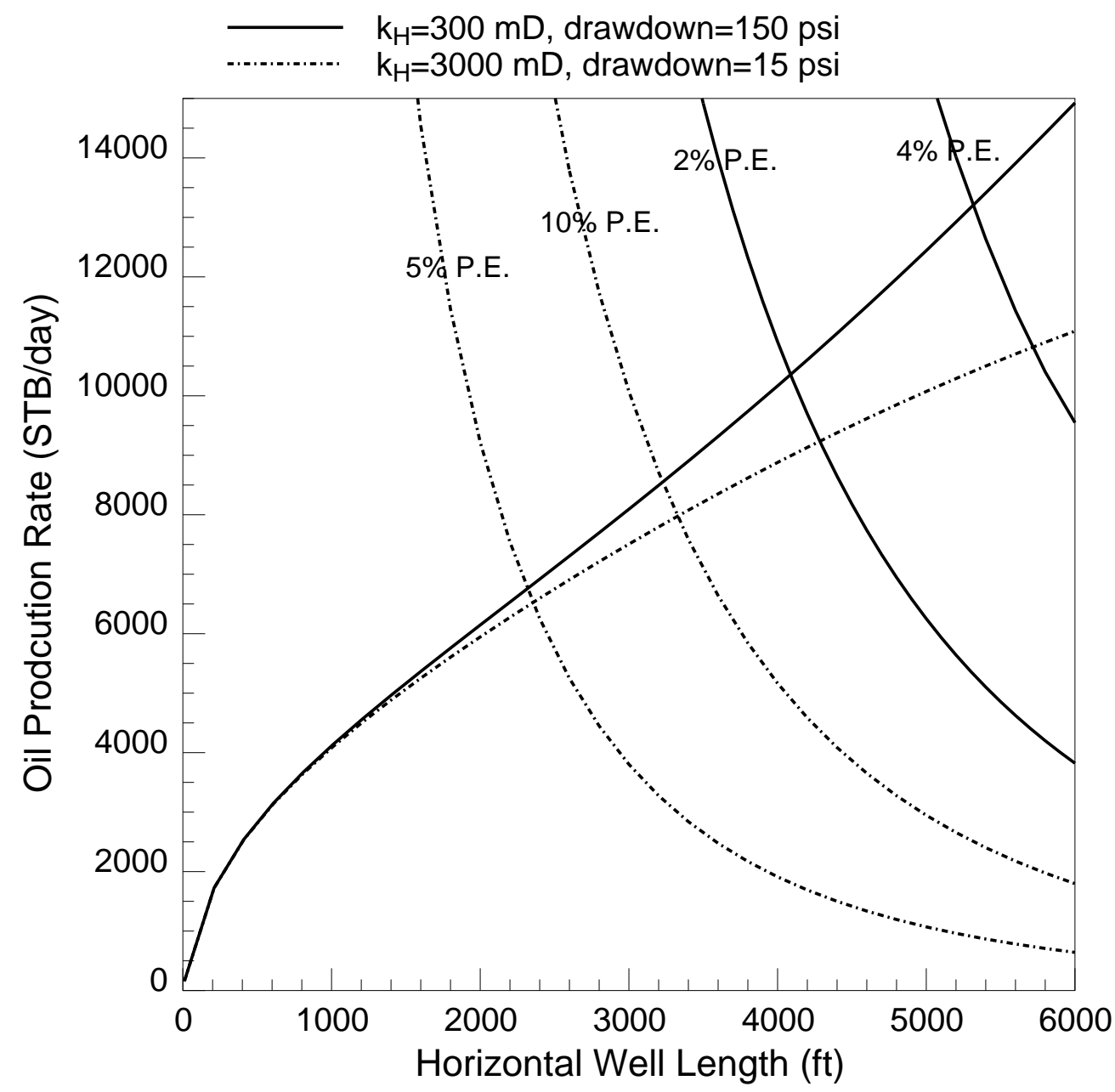

Figure 4.5: Effect of Reservoir Permeability on Wellbore Friction

\subsection{Two-Phase Flow in the Wellbore}

The frictional effects in the wellbore become more pronounced when there are twophases flowing. The effect of two-phase flow is illustrated by looking at a case where the GOR is $1000 \mathrm{SCF} / \mathrm{STB}$. The gas present is free gas and the GOR is assumed to be constant over the length of the horizontal well. Figure 4.6 shows a comparison of four different cases with a 15 psi drawdown in the reservoir. The four cases considered are: a) single phase oil flowing in the wellbore (the presence of gas is neglected), b) two-phase flow wellbore pressure drop computed by assuming no-slip homogeneous flow, c) two-phase flow wellbore pressure drop calculated by the Beggs \& Brill ${ }^{26}$ model and, d) friction is neglected in the wellbore. Let us look, for example, at the case when the oil rate is $8000 \mathrm{STB} /$ day. If friction is not considered in the wellbore, the calculations show that a horizontal well of length $3000 \mathrm{ft}$ is sufficient to produce this amount. But when frictional effects are considered, a $4500 \mathrm{ft}$ long well is required. Since friction is inevitable, a $3000 \mathrm{ft}$ well would produce only $6700 \mathrm{STB} /$ day. As the graph shows, frictional 
effects get worse when there are two phases flowing in the wellbore. The plot also shows that the P.E. is more when the Beggs \& Brill correlation is used than when a homogeneous flow model is used. If a new model that gives appropriate consideration to radial influx into the wellbore is used, then the pressure drop in the well would be higher.

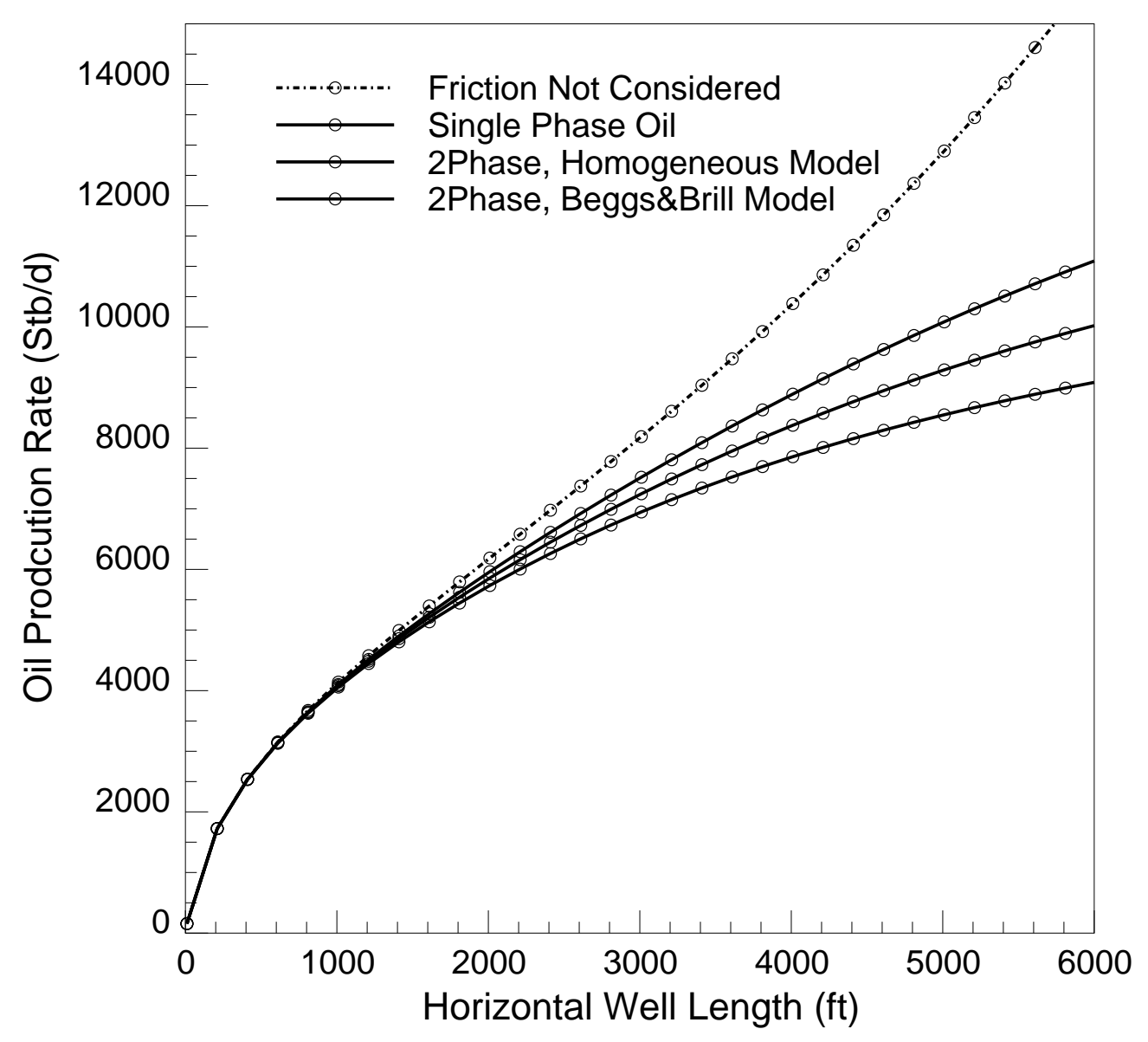

Figure 4.6: Effects of Two-Phase Flow in the Well on Wellbore Friction

\subsection{Frictional Effects on Water/Gas Breakthrough}

Figure 4.7 shows the effects of friction on breakthrough tendencies at the heel of the well. In this figure, the inflow from the reservoir into the well is shown for a $3000 \mathrm{ft}$ horizontal well. The inflow at the heel of the well is taken as a basis and the inflow at other well locations is shown as a fraction of the inflow at the heel. As the figure depicts, when the frictional effects are insignificant, the inflow into the well is uniform along the entire well length. But as frictional effects increase with increasing pipe roughness, the fluid inflow is highest at the heel and decreases towards the toe. The message from this figure is that ignoring frictional effects, when they are significant, can lead to under-estimation of breakthrough times for water and gas. 


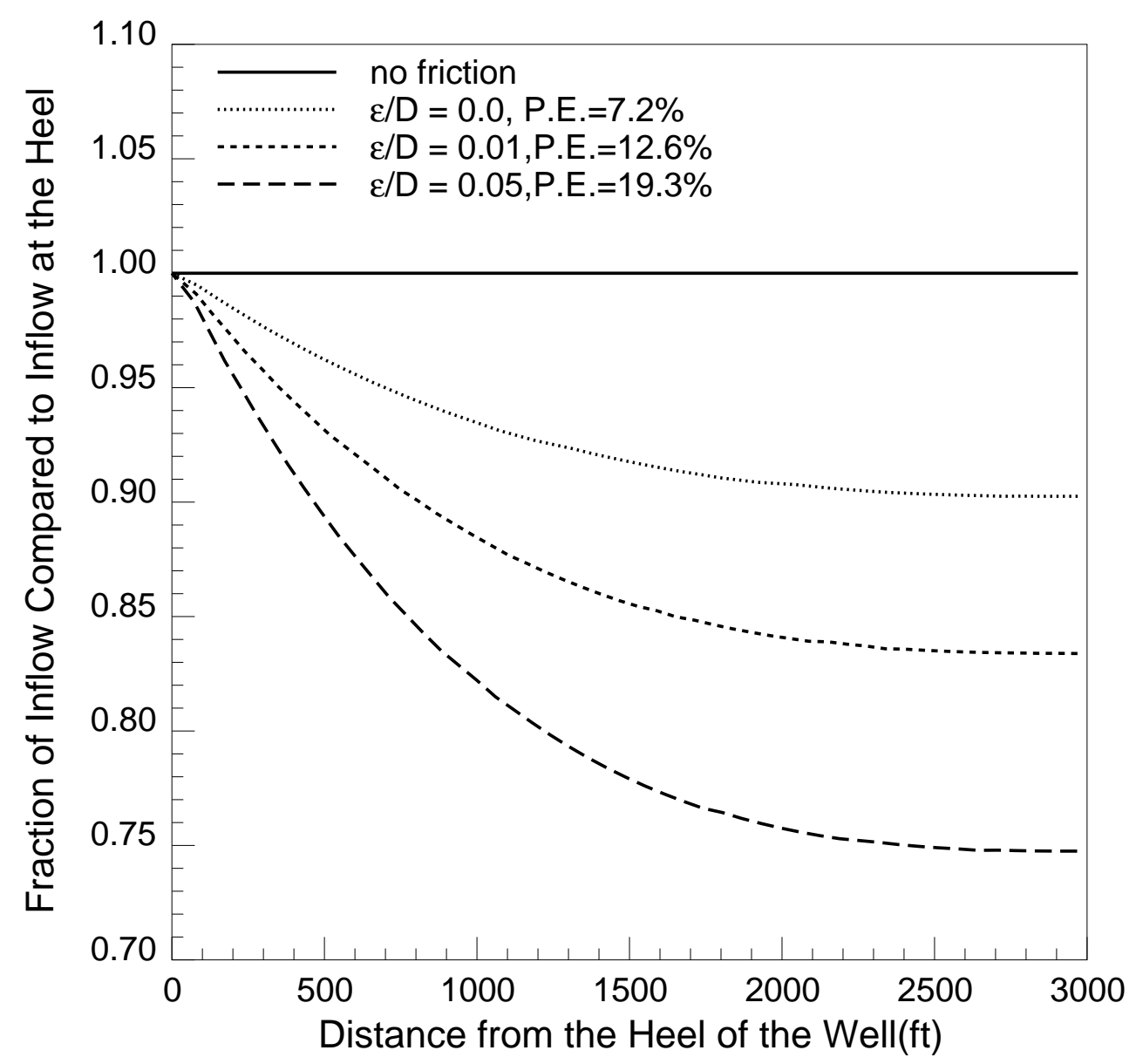

Figure 4.7: Early Breakthrough at the Heel of the Well due to Wellbore Friction

\subsection{Optimum Well Length}

Optimum well length is defined here as the length of the horizontal well in a given reservoir that gives maximum revenues, with consideration for frictional pressure drop in the wellbore. Because of the frictional effects, the incremental recovery of oil decreases with increasing well length. But the drilling and completions costs increase almost linearly as the well length is increased. Because of this, there will be a certain length of the well which maximizes revenues.

The total income from a well is given as a product of oil price and flow rate produced from the well.

Total Income $=\sum_{i=1}^{t} \$_{\text {oil }}^{i} Q_{w, \text { fric }}^{i} \frac{1}{(1+I)^{i}}$ 
$Q_{w, f r i c}^{i}$ is the production rate from the well in STB/day and $\$_{o i l}^{i}$ is the oil price in \$/STB at time $i$. $I$ is the interest rate and $t$ is the production time in days.

Total expenses for the well for this simplified case are the sum of the cost of drilling and completing the well and the operating cost.

Total Expenses $=C_{F}+C_{H} L+C_{M} t$

where $C_{F}$ is the fixed cost to drill vertical, $C_{H}$ is the cost to drill one foot of horizontal well, and $C_{M}$ is the operating cost per day of operation.

Net Revenue, $\mathrm{R}=$ Total Income - Total Expenses.

$R=\left[\sum_{i=1}^{t} \$_{\text {oil }}^{i} Q_{\text {fric }}^{i} \frac{1}{(1+I)^{i}}\right]-C_{F}-C_{H} L-C_{M} t$

For the sake of simplicity, we assume that the flow is at steady state so that the flow rate remains constant with time and we also assume that oil price is constant in the frame work of our time. Then Eq.(4.17) becomes,

$R=\$_{\text {oil }} Q_{w, f r i c} F(t, I)-C_{F}-C_{H} L-C_{M} t$

where

$F(t, I)=\sum_{i=1}^{t} \frac{1}{(1+I)^{i}}$

Under the special case when interest rate $I=0, F(t, I)$ will be equal to $t$.

Dividing both sides by $C_{F}$, we get:

$\frac{R}{C_{F}}=\frac{\$_{o i l} Q_{w, f r i c} F(t, I)}{C_{F}}-1-\frac{C_{H} L}{C_{F}}-\frac{C_{M} t}{C_{F}}$

The above equation can be non-dimensionalized using the following definitions:

Dimensionless net revenue, $R_{D}=\frac{R}{C_{F}}$

Dimensionless production rate, $Q_{D}=\frac{\$_{\text {oil }} Q_{w, \text { fric }} F(t, I)}{C_{M} t}$ 
Dimensionless well length, $L_{D}=\frac{C_{H} L}{C_{F}}$

Dimensionless production time, $t_{D}=\frac{C_{M} t}{C_{F}}$

With these definitions Eq.(4.20) becomes:

$R_{D}=Q_{D} t_{D}-1-L_{D}-t_{D}$

Taking the derivative with respect to $L_{D}$ on both sides and setting $\frac{d R_{D}}{d L_{D}}=0$ leads to,

$t_{D} \frac{d Q_{D}}{d L_{D}}=1$

Using the above equation, we can obtain the optimum length of a horizontal well for the cases of laminar and turbulent flow in the wellbore.

With laminar flow in the wellbore, the optimum well length is given by the following equation: (see Appendix B for details)

$L_{o p t}=\frac{1}{\sqrt{J_{s} R_{w}}} \cosh ^{-1}\left(\sqrt{\frac{\$_{o i l} J_{s} \Delta p_{0} F(t, I)}{C_{H}}}\right)$

where $R_{w}$ is the resistance in the wellbore. Eq.(4.23) shows that the optimum well length decreases as flow resistance in the wellbore increases. Also the optimum well length increases as the oil price increases, because with a higher oil price an additional barrel of oil produced due to a longer well is worth more.

When there is turbulent flow in the wellbore, the optimum well length is calculated using the following relationship:

$\frac{d Q}{d L}=\left[\frac{1}{J_{s} \Delta p_{0}}+J_{s} \frac{d p_{w}}{d x}(Q) M^{\prime}\right]^{-1}$

where,

$M^{\prime}=\int_{0}^{Q} \frac{1}{\left[2 J_{s} \int_{Q}^{q} \frac{d p_{w}}{d x} d \varepsilon+\left[J_{s} \Delta p_{0}\right]^{2}\right]^{\frac{3}{2}}} d q_{w}$

Using Eq.(4.24) and Eq.(4.22), we can calculate the flow rate from the well with optimum length. $L_{\text {opt }}$ can then be calculated using this flow rate in Eq.(4.8). 
Results of an example problem to show the effects of friction on the optimum well length are shown in Figure 4.8. This figure shows that when friction is neglected, the production from the well increases at a constant rate with an increase in the well length. But when turbulent flow in the wellbore is considered, then after a certain well length revenues start to drop. The optimum well length in this example is about $1600 \mathrm{ft}$.

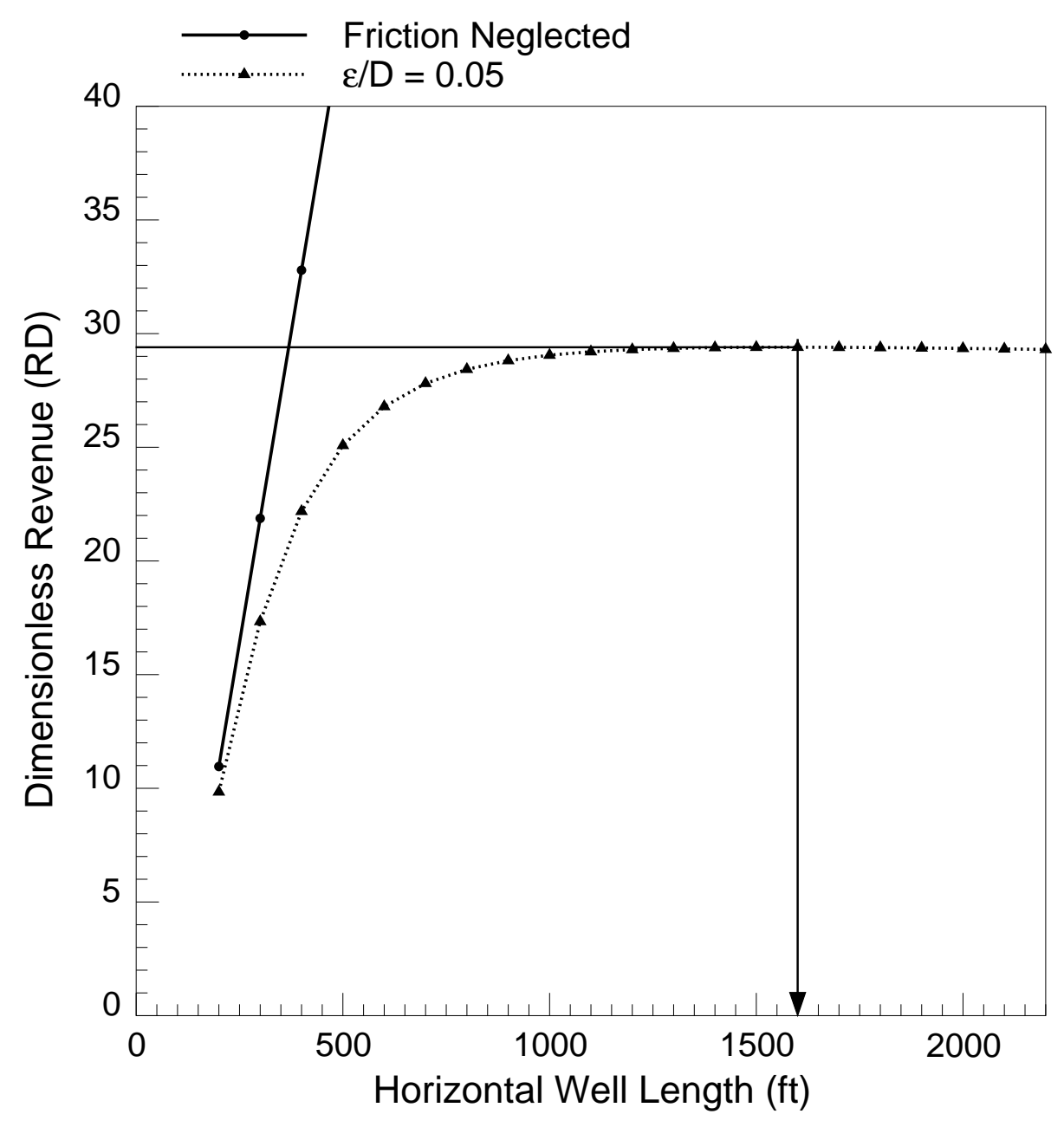

Figure 4.8: Optimum Well Length Calculation

The radius of the well can also be optimized along with its length. The costs involved in changing the radius of the well are not very direct. We need to account for pipe costs, drilling costs, mud costs and various others. If all those parameters are available, then a similar analysis can also yield the optimum well radius. 


\subsection{Future Work}

In the future, we plan to extend this model to transient flow in the reservoir. We also plan to couple our model to a three phase, three dimensional numerical simulator and develop a procedure for determining optimum well length for more realistic problems.

\subsection{Conclusions}

1. A flexible reservoir/wellbore coupling model was developed to account for frictional pressure drop in horizontal wells.

2. The ratio of wellbore pressure drop to reservoir drawdown gives a good indication of the frictional effects on well productivity.

3. Ignoring pressure drop in the wellbore can give higher estimates for well productivity and later estimates for breakthrough time.

4. Errors in productivity calculations due to ignoring frictional effects increase with increasing well length, flow rates, wellbore roughness and reservoir permeabilities; and they decrease with higher drawdown or higher fluid viscosities.

5. A methodology to calculate the optimum well length for horizontal wells was also developed.

\section{Acknowledgments}

This work was supported by the US Department of Energy through Contract Number DFFG22-93BC14862. Support was also provided by the Stanford University Horizontal Well Affiliate Program.

\section{Nomenclature}

$C_{F} \quad$ Fixed cost for drilling \& completion of the vertical part of the well, $\$$

$C_{H} \quad$ Cost per unit length for drilling \& completion of the horizontal part of the well, $\$ / \mathrm{ft}$

$C_{M} \quad$ Operating cost per day of production, $\$ /$ day

D Diameter of the well, $\mathrm{ft}$

f Moody friction factor

F A specified function

I Annual interest rate

$J_{s} \quad$ Productivity index per unit length of the well, $\mathrm{rbbl} / \mathrm{day} / \mathrm{psi} / \mathrm{ft}$

$\mathrm{k} \quad$ Reservoir permeability, $\mathrm{mD}$

L Horizontal well length, $\mathrm{ft}$

$\mathrm{p}$ pressure, psi

P.E. Productivity error 
$\$_{\text {oil }} \quad$ Oil price per barrel, $\$ /$ STB

$q_{s} \quad$ Inflow into the well per unit length of the well

Q Oil production rate, rbbl/day

$q_{w} \quad$ Flow in the wellbore, rbbl/day

$\mathrm{R} \quad$ Net revenue, $\$$

$R_{w} \quad$ Resistance in the wellbore, $\mathrm{rbbl} / \mathrm{day}$

$\mathrm{t} \quad$ Production time, day

U Superficial oil flow rate in the well, cu.ft/sec

$\mathrm{x}$ Distance along the wellbore coordinate, $\mathrm{ft}$

\section{Greek Letters}

$\rho \quad$ Oil density, lbm/cu.ft

$\Delta p_{0} \quad$ Drawdown at the heel of the well, psi

\section{Subscripts}

$\begin{array}{ll}\text { H } & \text { Horizontal } \\ \text { V } & \text { Vertical } \\ \text { w } & \text { Well } \\ \text { e } & \text { External boundary } \\ \text { s } & \text { Specific } \\ \text { fric } & \text { Frictional effects considered } \\ \text { nof } & \text { Frictional effects not considered } \\ \text { opt } & \text { Optimum }\end{array}$

\section{References}

1. Giger, F.: "Reduction du nombre de puits par l'utilisation de forages horizontaux," Revue de I'Institut du Petrole, vol. 38, No 3, May-June 1983

2. Borisov, Ju. P.: "Oil Production Using Horizontal and Multiple Deviation Wells," Nedra, Moscow, 1964. Translated by J. Stauss, S.D. Joshi (ed.), Phillips Petroleum Co., the R \& D Library Translation, Bartlesville, Oklahoma, 1984

3. Giger, F.M., Reiss, L.H., and Jourdan, A.P.: "The Reservoir Engineering Aspect of Horizontal Drilling," paper SPE 13024 presented at the SPE 59 $9^{\text {th }}$ Annual Technical Conference and Exhibition, Houston, Texas, Sept. 16-19, 1984

4. Renard, G.I. and Dupuy, J.M.: "Influence of Formation Damage on the Flow Efficiency of Horizontal Wells," paper SPE 19414, presented at the Formation Damage Control Symposium, Lafayette, Louisiana, Feb. 22-23, 1990 
5. Joshi, S.D.: “Augmentation of Well Productivity Using Slant and Horizontal Wells," Journal of Petroleum Technology, pp. 729-739, June 1988

6. Economides, M.J., Hill, A.D., and Economides, C.E.: Petroleum Production Systems, PTR Prentice Hall, pp.33, 1994

7. Mutalik, P.N., Godbole, S.P., and Joshi, S.D.: "Effect of Drainage Area Shapes on Horizontal Well Productivity," paper SPE 18301, presented in the SPE $63^{\text {rd }}$ Annual Technical Conference, Houston, Texas, Oct. 2-5, 1988

8. Kuchuk, F.J., Goode, P.A., Brice, B.W., Sherrard, D.W., and Thambynayagam, R.K.M.: "Pressure Transient Analysis and Inflow Performance for Horizontal Wells," paper SPE 18300, presented in the SPE $63^{\text {rd }}$ Annual Technical Conference and Exhibition, Houstan, Texas, Oct. 2-5, 1988

9. Babu, D.K. and Odeh, A.S.: "Productivity of a Horizontal Well," SPE Reservoir Engineering, pp. 417-421, November 1989

10. Gringarten, A.C., Ramey, H.J. Jr. and Raghavan, R.: "Unsteady-State Pressure Distributions Created by a Well with Single Infinite-Conductivity Vertical Fracture," SPE Journal, pp. 300-347, August 1974

11. Clonts, M.D. and Ramey, H.J. Jr.: "Pressure Transient Analysis for Wells With Horizontal Drainholes," paper SPE 15116 presented at the SPE California Regional Meeting, Oakland, April 2-4, 1986

12. Goode, P.A. and Thambynayagam, R.K.M.: "Pressure Drawdown and Buildup Analysis of Horizontal Wells in Anisotropic Media," SPEFE (Dec. 1987) 683-97; Trans., AIME, 283.

13. Kuchuk, F.J., Goode, P.A., Wilkinson, D.J. and Thambynayagam, R.K.M.: "Pressure Transient Behavior of Horizontal Wells With and Without Gas Cap or Aquifer," paper SPE 17413, presented at the SPE California Regional Meeting, Long Beach, California, March 23-35, 1988

14. Daviau, F., Mouronval, G., Bourdarot, G. and Curutchet, P.: "Pressure Analysis for Horizontal Wells," SPE Formation Evaluation, pp. 716-724, December 1988

15. Ozkan, E., Raghavan, R. and Joshi, S.D.: "Horizontal-Well Pressure Analysis," SPE Formation Evaluation, pp. 567-575, December 1989

16. Odeh, A.S. and Babu, D.K.: "Transient Flow Behavior of Horizontal Wells: Pressure Drawdown and Buildup Analysis," SPE Formation Evaluation, pp. 7-15, 1990

17. Dikken, B.J.: "Pressure Drop in Horizontal Wells and its Effects on Production Performance," Journal of Petroleum Technology, 1426-1433, Nov. 1990 
18. Novy, R.A.: "Pressure Drop in Horizontal Wells: When Can They Be Ignored?," SPE Reservoir Engineering, pp. 29-35, February 1995

19. Ozkan, E., Sarica, C., and Haciislamoglu, M.: "Effect of Conductivity on Horizontal Well Pressure Behavior," SPE Advanced Technical Series, Vol. 3, No. 1, 85-93, 1995

20. Landman, M.J.: "Analytical Modeling of Selectively Perforated Horizontal Wells," Journal of Petroleum Science \& Engineering, 10, 179-188, 1994

21. Stone, T.W. and Kristoff, B.J.: "A Comprehensive Wellbore/Reservoir Simulator," paper SPE 18419 presented at the SPE Symposium on Reservoir Simulation, Houstan, Feb. 6-8, 1989

22. Islam, M.R. and Chakma, A.: "Comprehensive Physical and Numerical Modeling of a Horizontal Well," paper SPE 20627 presented at the SPE 65th Annual Technical Conference and Exhibition, New Orleans, LA, 1990

23. Folefac, A.N., Archer, J.S., and Issa, R.I.: "Effect of Pressure Drop Along Horizontal Wellbores on Well Performance," paper SPE 23094 presented at the Offshore Europe Conference, Aberdeen, 1991

24. Brekke, K., Johansen, T.E., and Olufsen, R.: "A New Modular Approach to Comprehensive Simulation of Horizontal Wells," paper SPE 26518 presented at the SPE 68th Annual Technical Conference and Exhibition, Houstan, TX, 1993

25. Jain, A.K.: “Accurate Explicit Equation for Friction Factor,” J. Hydraulics Div. ASCE (1976) $102,674-677$

26. Beggs, H.D. and Brill, J.P.: "A Study of Two-Phase Flow in Inclined Pipes," Journal of Petroleum Technology, 607-617, May, 1973 


\section{Appendix A:}

\section{Flow Rate vs. Well Length}

Mass balance gives the following equation for flow in the wellbore:

$\frac{d}{d x} q_{w}(x)=-q_{s}(x)$

where $q_{w}(x)$ is the flow rate in the wellbore and it varies along the length of the well. Combining Eq.(4.1) and Eq.(4.A1), we have

$\frac{d}{d x} q_{w}(x)=-J_{s}\left[p_{e}-p_{w}(x)\right]$

Differentiating Eq.(4.A2), with constant $J_{s}$ and $p_{e}$, results in

$\frac{d^{2} q_{w}(x)}{d x^{2}}=J_{s} \frac{d p_{w}(x)}{d x}$

Multiplying both sides by $\frac{d q_{w}}{d x}$, as Landman ${ }^{20}$ did, we get

$\frac{d q_{w}(x)}{d x} \frac{d}{d x}\left[\frac{d q_{w}(x)}{d x}\right]=J_{s} \frac{d p_{w}(x)}{d x} \frac{d q_{w}(x)}{d x}$

$\frac{1}{2} \frac{d}{d x}\left[\frac{d q_{w}}{d x} \frac{d q_{w}}{d x}\right]=J_{s} \frac{d p_{w}(x)}{d x} \frac{d q_{w}(x)}{d x}$

$d\left[\left(\frac{d q_{w}}{d x}\right)^{2}\right]=2 J_{s} \frac{d p_{w}(x)}{d x} d q_{w}(x)$

Integrating the above equation, we obtain

$$
\begin{gathered}
\int_{q_{w}=Q}^{q_{w}=q_{w}} d\left[\left(\frac{d q_{w}}{d x}\right)^{2}\right]=2 J_{s} \int_{q_{w}=Q}^{q_{w}=q_{w}} \frac{d p_{w}(x)}{d x} d q_{w}(x) \\
{\left[\frac{d q_{w}}{d x}\right]_{q_{w}}^{2}-\left[\frac{d q_{w}}{d x}\right]_{q_{w}=Q}^{2}=2 J_{s} \int_{Q}^{q_{w}} \frac{d p_{w}(x)}{d x} d q_{w}(x)}
\end{gathered}
$$

Using boundary condition I, defined as: 


$$
\begin{aligned}
& {\left[\frac{d q_{w}}{d x}\right]_{x=0}=\left[\frac{d q_{w}}{d x}\right]_{q_{w}=Q}=-J_{s}\left[p_{e}-p_{w, 0}\right]} \\
& =-J_{s} \Delta p_{0}
\end{aligned}
$$

Eq.(4.A8) becomes

$$
\begin{aligned}
& {\left[\frac{d q_{w}}{d x}\right]^{2}-\left[-J_{s} \Delta p_{0}\right]^{2}=2 J_{s} \int_{Q}^{q_{w}} \frac{d p_{w}(x)}{d x} d q_{w}(x)} \\
& \frac{d q_{w}}{d x}=-\sqrt{2 J_{s} \int_{Q}^{q_{w}} \frac{d p_{w}(x)}{d x} d q_{w}(x)+\left[J_{s} \Delta p_{0}\right]^{2}}
\end{aligned}
$$

The choice of negative for the square root in the above equation comes from the fact that $q_{w}$ increases as $\mathrm{x}$ decreases. Using boundary condition II, defined as

$$
q_{w, x=L}=0
$$

yields,

$$
\begin{aligned}
& \int_{x=x}^{x=L} d x=-\int_{q_{w}=q_{w}}^{q_{w}=0} \frac{d q_{w}(x)}{\sqrt{2 J_{s} \int_{Q}^{q_{w}}\left[\frac{d p_{w}}{d x}\right] d q_{w}(x)+\left[J_{s} \Delta p_{0}\right]^{2}}} \\
& L-x=\int_{0}^{q_{w}(x)} \frac{d q_{w}(x)}{\sqrt{2 J_{s} \int_{Q}^{q_{w}}\left[\frac{d p_{w}}{d x}\right] d q_{w}(x)+\left[J_{s} \Delta p_{0}\right]^{2}}}
\end{aligned}
$$

Since at the heel, $q_{w}(x=0)=Q$, the above equation can be expressed as,

$$
L=\int_{0}^{Q} \frac{d q_{w}(x)}{\sqrt{2 J_{s} \int_{Q}^{q_{w}}\left[\frac{d p_{w}}{d x}\right] d q_{w}(x)+\left[J_{s} \Delta p_{0}\right]^{2}}}
$$




\section{Appendix B: Optimum Well Length}

\section{Laminar Flow in the Wellbore:}

For this case, Dikken derived an expression for the flow rate from the well:

$$
Q_{w, f r i c}=\sqrt{\frac{J_{s}}{R_{w}}} \tanh \left(L \sqrt{J_{s} R_{w}}\right) \Delta p_{0}
$$

where $R_{w}$ is the resistance to flow in the wellbore.

For a smooth pipe, $R_{w}$ was defined as,

$$
R_{w}=2.921 \times 10^{-12}\left(\frac{\mu D}{\rho}\right)^{0.25} \frac{\rho}{\pi^{1.75} D^{5}}
$$

Assuming that $J_{s}$ is a weak function of L, we get

$$
\frac{d Q_{w, \text { fric }}}{d L}=\sqrt{\frac{J_{s}}{R_{w}}} \sqrt{J_{s} R_{w}} \sec h^{2}\left(L \sqrt{J_{s} R_{w}}\right) \Delta p_{o}
$$

From the definition of dimensionless variables, we have

$$
\frac{d Q_{D}}{d L_{D}} t_{D}=\frac{d Q_{w, f r i c}}{d L} t \frac{\$_{o i l} F(t, I)}{C_{M} t} \frac{C_{M}}{C_{F}} \frac{C_{F}}{C_{H}}=\frac{d Q_{w, f r i c}}{d L} \frac{\$_{o i l} F(t, I)}{C_{H}}
$$

Substituting the above equation in Eq.(4.22), the optimum well length is obtained,

$$
L_{o p t}=\frac{1}{\sqrt{J_{s} R_{w}}} \cosh ^{-1}\left(\sqrt{\frac{\$_{o i l} J_{s} \Delta p_{0} F(t, I)}{C_{H}}}\right)
$$

\section{Turbulent Flow in the wellbore:}

Eq.(4.A15) defines the flow in the system when there is turbulent flow in the wellbore. We need the derivative of Eq.(4.A15) with respect to. L to calculate the optimum well length. We use Leibnitz's rule to accomplish this. Leibnitz's rule states:

If $I(\alpha)=\int_{a(\alpha)}^{b(\alpha)} f(x, \alpha) d x$, then 


$$
\frac{d I}{d \alpha}=I^{\prime}(\alpha)=\int_{a(\alpha)}^{b(\alpha)} \frac{\partial f(x, \alpha)}{\partial \alpha} d x+f[b(\alpha), \alpha] \frac{d b}{d \alpha}-f[a(\alpha), \alpha] \frac{d a}{d \alpha}
$$

Taking the derivative of Eq.(4.A15) with respect to L, we get

$$
1-\int_{0}^{Q} \frac{\partial}{\partial L}\left[\frac{1}{\sqrt{2 J_{s} \int_{Q}^{q_{w}} \frac{d p_{w}}{d x} d \varepsilon+\left[J_{s} \Delta p_{0}\right]^{2}}}\right] d q_{w}(x)-\frac{1}{\left[J_{s} \Delta p_{0}\right]} \frac{d Q}{d L}=0
$$

In the above equation, $\varepsilon$ is the variable of integration.

If we set $\mathrm{M}=\frac{\partial}{\partial L}\left[\frac{1}{\sqrt{2 J_{S} \int_{Q}^{q_{w}} \frac{d p_{w}}{d x} d \varepsilon+\left[J_{s} \Delta p_{0}\right]^{2}}}\right]$, and perform the differentiation using the Leibnitz's rule on the integral, we obtain

$$
\begin{aligned}
& M=\frac{\partial}{\partial L}\left[\frac{1}{\left.\sqrt{2 J_{S} \int_{Q}^{q_{w}} \frac{d p_{w}}{d x} d \varepsilon+\left[J_{s} \Delta p_{0}\right]^{2}}\right]}\right] \\
& =-\frac{1}{2} \frac{1}{\left[2 J_{s} \int_{Q}^{q_{w}} \frac{d p_{w}}{d x} d \varepsilon+\left[J_{s} \Delta p_{0}\right]^{2}\right]^{\frac{3}{2}}} *\left(2 J_{S}\left[\int_{Q}^{q_{w}} \frac{\partial}{\partial L}\left[\frac{d p_{w}}{d x}\right] d \varepsilon+\frac{d p_{w}}{d x}\left(q_{w}\right) \frac{d q_{w}}{d L}-\frac{d p_{w}}{d x}(Q) \frac{d Q}{d L}\right]\right)
\end{aligned}
$$

In the above equation, we can write

$$
\frac{\partial}{\partial L}\left[\frac{d p_{w}}{d x}\right]=\frac{\partial}{\partial \varepsilon}\left[\frac{d p_{w}}{d x}\right] \frac{\partial \varepsilon}{\partial L}
$$

Since $q_{w}$ and $\varepsilon$ are variables of integration,

$$
\frac{d q_{w}}{d L}=\frac{\partial \varepsilon}{\partial L}=0
$$


Using above relations in Eq.(4.B8), we obtain

$\frac{d Q}{d L}\left[\frac{1}{J_{s} \Delta p_{0}}+J_{s} \frac{d p_{w}}{d x}(Q) M^{\prime}\right]=1$

where,

$$
M^{\prime}=\int_{0}^{Q} \frac{1}{\left[2 J_{s} \int_{Q}^{q_{w}} \frac{d p_{w}}{d x} d \varepsilon+\left[J_{s} \Delta p_{0}\right]^{2}\right]^{\frac{3}{2}}} d q_{w}
$$

Which implies that,

$\frac{d Q}{d L}=\left[\frac{1}{J_{s} \Delta p_{0}}+J_{s} \frac{d p_{w}}{d x}(Q) M^{\prime}\right]^{-1}$

Using the above equation along with Eq.(4.22), we can calculate the flow rate from the well with optimum length. $L_{o p t}$ can then be determined using this flow rate in Eq.(4.A15).

\section{SI Metric Conversion Factors}

$\begin{array}{ll}\mathrm{bbl} \times 1.589873 \mathrm{E}-01 & =\mathrm{m}^{3} \\ \mathrm{cP} \times 1.0 \mathrm{E}+00 & =\mathrm{mPa} . \mathrm{s} \\ \mathrm{ft} \times 3.048 \mathrm{E}-01 & =\mathrm{m} \\ \mathrm{lbm} \times 4.535924 \mathrm{E}-01 & =\mathrm{kg} \\ \operatorname{psi} \times 6.894757 \mathrm{E}+00 & =\mathrm{kPa}\end{array}$




\title{
5. Effects of Wellbore Friction Under Transient Conditions
}

\author{
by Raju Penmatcha* and Khalid Aziz
}

\begin{abstract}
We have developed an analytical solution to describe the three dimensional, transient flow in a reservoir with horizontal wells that have infinitely conductive wellbores. A methodology is also developed that couples frictional pressure drop in the wellbore to the analytical solution. Using this model, we can evaluate the effects of wellbore friction on horizontal well productivity under transient flow conditions.
\end{abstract}

\subsection{Introduction}

In the preceding Chapter, we demonstrated the effects of friction in the wellbore when there is steady state flow both in the reservoir and the wellbore. In this section, we develop an analytical solution that can be used to study frictional effects under transient conditions. We develop a methodology to analytically describe three dimensional flow in a reservoir with horizontal wells that have infinitely conductive wellbores. The base solution that we use is the the one derived by Babu \& Odeh [1] for horizontal well productivity calculations in a box shaped reservoir. This model assumes that the reservoir is homogeneous (can be anisotropic), single phase oil flow, all the wells are horizontal wells in the $\mathrm{x}$ or $\mathrm{y}$ direction, and the reservoir is box shaped with no-flux boundaries. Babu \& Odeh's solution assumes that the flux along the well is uniform. Since uniform flux is not always possible, they make this assumption purely for mathematical convenience. We derive a solution for an infinitely conductive well using their base solution. The principle of super-position in space and time is used to arrive at this solution.

\subsection{Babu \& Odeh's Solution for Horizontal Well Productivity}

Babu \& Odeh [1] have developed a general solution for the productivity of a horizontal well in a box-shaped drainage volume. As shown in Figure 5.1, the reservoir contains a horizontal well of radius $r_{w}$ and length L. All the six external boundaries of the drainage volume are of no-flow type. The drainage volume has a height $h$, length a and width $b$. As shown in the figure, the well extends from $(\mathrm{x} 0, \mathrm{y} 1, \mathrm{z} 0)$ to $(\mathrm{x} 0, \mathrm{y} 2, \mathrm{z} 0)$ and is parallel to the y-axis. The well can be placed anywhere in the drainage volume but must be parallel to one of the co-ordinate axes and it can be of any length $(L \leq b)$. Reservoir should be homogeneous but can be anisotropic. Porosity $\phi$ is constant. $k_{x}, k_{y}$, and $k_{z}$ are the reservoir permeabilities in $\mathrm{x}, \mathrm{y}$ and $\mathrm{z}$ directions. There should be single phase, slightly compressible fluid in the reservoir. Initially at time $t=0$, the pressure is uniform throughout the reservoir and is equal to $p_{i}$. At time $t=0^{+}$, fluid is withdrawn from the well at a constant rate of $\mathrm{q}$.

\footnotetext{
${ }^{*}$ The analytical solution developed in this work is a result of our discussions with D.K. Babu, Mobil
} 
Under these circumstances, they have developed a solution for pressure at any time $(t<0)$, or at any position $(0 \leq x \leq a, 0 \leq y \leq b, 0 \leq z \leq h)$ in the system. More details about this model can be found in their paper [1].
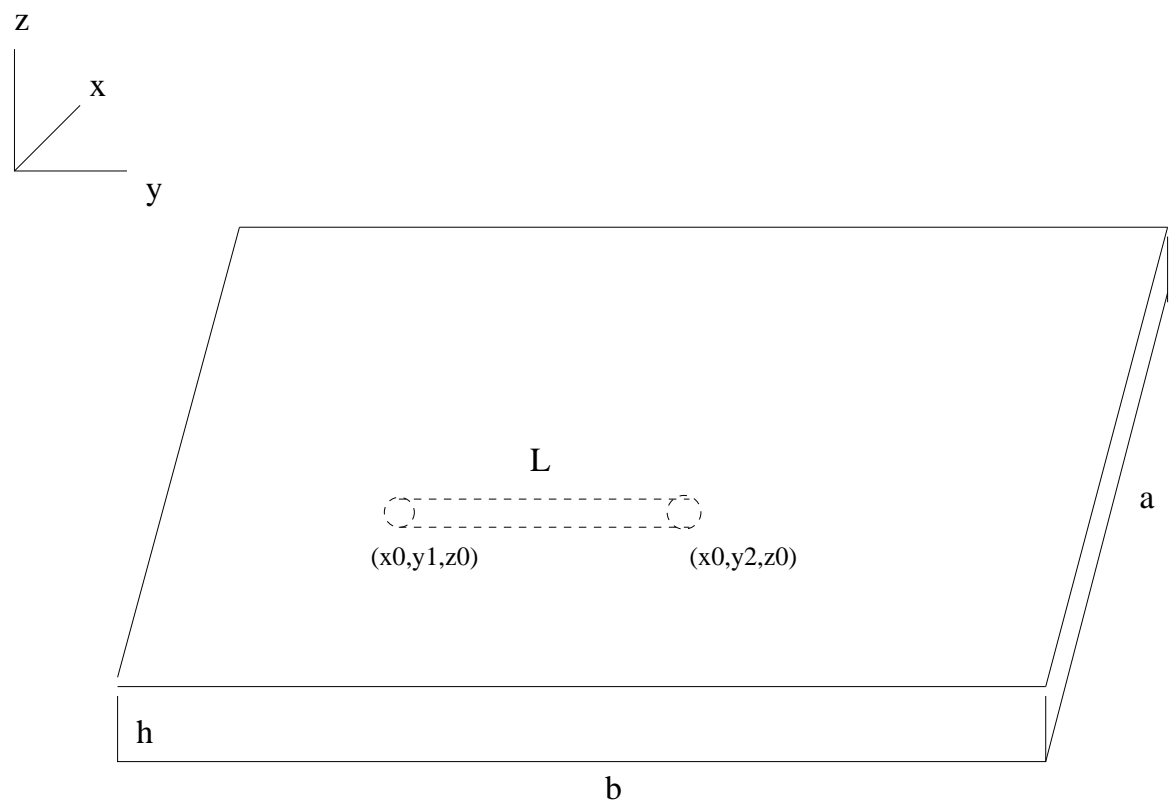

Figure 5.1: Horizontal well in a box-shaped reservoir (Babu and Odeh [1])

The flux along the well is assumed to be uniform in the model described above. But the flux can be uniform only if the well is fully penetrating resulting in $2 \mathrm{D}$ type of flow. When the well is partially penetrating, the pressure support from the reservoir at the two ends of the well would result in higher flux at the ends of the well as compared to the flux at the center of the well. For the cases where the pressure drop in the wellbore is not very significant compared to the drawdown, an infinitely conductive wellbore is a far better assumption than a uniform flux wellbore. In this section, we develop a solution for this case using Duhamel's principle and the uniform flux solution of Babu \& Odeh.

The uniform flux solution by Babu \& Odeh is given as:

$$
\triangle p \equiv p_{i}-p(x, y, z, t)=\left[\frac{886.9 B \mu q}{a b h L \alpha}\right] \int_{0}^{t} \int_{y_{1}}^{y_{2}}\left(S_{1} \cdot S_{2} . S_{3}\right) d y_{0} d \tau
$$

where $S_{1}, S_{2}$, and $S_{3}$ are instantaneous point sink functions (Green's functions) located at $\left(x_{0}, y_{0}, z_{0}\right)$ and satisfying no-flow boundary conditions at $\mathrm{x}=0, \mathrm{a} ; \mathrm{y}=0, \mathrm{~b}$; and $\mathrm{z}=0, \mathrm{~h} . x_{0}, y_{0}$, and $z_{0}$ are the $\mathrm{x}, \mathrm{y}$ and $\mathrm{z}$ coordinates at the center of the well. $S_{1}, S_{2}$, and $S_{3}$ are defined as,

$$
\begin{gathered}
S_{1} \equiv S_{1}\left(x, x_{0}, \tau\right) \equiv 1+2 \sum_{n=1}^{\infty} \cos \frac{n \pi x}{a} \cos \frac{n \pi x_{0}}{a} \exp \left[-\frac{n^{2} \pi^{2} k_{x} \tau}{\alpha a^{2}}\right] \\
S_{2} \equiv S_{2}\left(y, y_{0}, \tau\right) \equiv 1+2 \sum_{m=1}^{\infty} \cos \frac{m \pi y}{b} \cos \frac{m \pi y_{0}}{b} \exp \left[-\frac{m^{2} \pi^{2} k_{y} \tau}{\alpha b^{2}}\right] \\
S_{3} \equiv S_{3}\left(z, z_{0}, \tau\right) \equiv 1+2 \sum_{l=1}^{\infty} \cos \frac{l \pi z}{h} \cos \frac{l \pi z_{0}}{h} \exp \left[-\frac{l^{2} \pi^{2} k_{z} \tau}{\alpha h^{2}}\right]
\end{gathered}
$$


where $\alpha=157.952 \phi \mu c_{t}$, days

They carried out the integration to obtain the pressure drop at any point $(\mathrm{x}, \mathrm{y}, \mathrm{z})$ in the reservoir at time $t$.

$$
\triangle p=\left(p_{i}-p(x, y, z, t)\right)=\left[\frac{886.9 B q \mu}{a b h \alpha}\right][t+\text { otherterms }]
$$

The missing terms in the above equation can be found in Ref.[1]. Eq. 5.5 can also be written as,

$$
\triangle p=\left(p_{i}-p\right)=q \cdot F(t)
$$

\subsection{Analytical Solution: Infinite Conductive Well}

In this section we develop an analytical solution, for a horizontal well with single or multi-laterals placed anywhere in a box-shaped reservoir with sealed boundaries. We use the principle of super-position in space and time to accomplish this task. Let us divide the original well into $\mathrm{n}$ segments. Well segment 1 is assumed to be closest to the heel of the well, while segment $\mathrm{n}$ is closest to the toe of the well. We can now apply the uniform flux solution for each well segment as given by Eq. 5.6. The interaction between each segment can be taken into account by superposition of pressures in space. This type of superposition is possible because of the linearity of the flow in the porous media. Let the mid-point of every segment be the pressure node for that segment. At time $\triangle t$, we can write:

$$
\triangle p_{1}=q_{1} F_{1,1}(\triangle t)+q_{2} F_{1,2}(\triangle t)+\ldots .+q_{n} F_{1, n}(\triangle t)
$$

where $\triangle p_{1}=\left(p_{i}-p(x 0, y m 1, z 0)\right)$ and it gives the pressure drop at the pressure node of well segment 1 due to the flow from itself and all other well segments. The term $F_{m, n}$ in

the above equation stands for the effect of well segment $n$ on well segment $m$. We can write similar equations for all the other segments. For well segment $\mathrm{m}$, for example,

$$
\triangle p_{m}=q_{1} F_{m, 1}(\triangle t)+q_{2} F_{m, 2}(\triangle t)+\ldots .+q_{n} F_{m, n}(\triangle t)
$$

The unknowns in this problem are 'n' q's at the nodes of the $n$ segments. We need 'n' equations to get a closed form for the system of equations. By taking the pressure difference between node 1 and every other node we can form (n-1) equations. For example, the pressure difference between node 1 and node $\mathrm{m}$ is given as:

$$
\begin{array}{r}
\triangle p_{1}-\triangle p_{m}=p_{m}-p_{1}=q_{1}\left[F_{1,1}(\triangle t)-F_{m, 1}(\triangle t)\right]+q_{2}\left[F_{1,2}(\triangle t)-F_{m, 2}(\triangle t)\right] \\
+\ldots+q_{n}\left[F_{1, n}(\triangle t)-F_{m, n}(\triangle t)\right]
\end{array}
$$

Because of the infinite conductivity assumption, pressure is constant in the wellbore. Hence the term on the left hand side of Eq. 5.9 is equal to zero. At this point there is only one more equation needed to close the system of equations. Depending on the well constraint it can be either total flow rate specified or BHP specified.

If the total rate $q_{T}$ is specified, then the last equation will be

$$
q_{T}=q_{1}+q_{2}+\ldots .+q_{n}
$$


If the BHP pressure is specified, then the last equation will be

$$
\triangle p_{1}=p_{i}-p_{1}=q_{1} F_{1,1}(\triangle t)+q_{2} F_{1,2}(\triangle t)+\ldots .+q_{n} F_{1, n}(\triangle t)
$$

where $p_{1}$ is the pressure at the node of segment 1 and is equal to the specified BHP.

Solving the system of $\mathrm{n}$ equations given above, we can calculate the q's from each node for the first time step. Superposition in space was applied in the above set of equations. Now we need to apply superposition in time, to march our solution through time. It is done next.

Let us assume that all time steps are of equal length and are equal to $\triangle t$. Based on that, when $t=3 \triangle t$ (at the end of third time step), we have

For well segment 1:

$$
\begin{array}{r}
\triangle p_{1}=q_{1} F_{1,1}(3 \triangle t)+\triangle_{2} q_{1} F_{1,1}(2 \triangle t)+\triangle_{3} q_{1} F_{1,1}(\triangle t) \\
+q_{2} F_{1,2}(3 \triangle t)+\triangle_{2} q_{2} F_{1,2}(2 \triangle t)+\triangle_{3} q_{2} F_{1,2}(\triangle t) \\
+\ldots+q_{n} F_{1, n}(3 \triangle t)+\triangle_{2} q_{n} F_{1, n}(2 \triangle t)+\triangle_{3} q_{n} F_{1, n}(\triangle t)
\end{array}
$$

For well segment 2:

$$
\begin{array}{r}
\triangle p_{2}=q_{1} F_{2,1}(3 \triangle t)+\triangle_{2} q_{1} F_{2,1}(2 \triangle t)+\triangle_{3} q_{1} F_{2,1}(\triangle t) \\
+q_{2} F_{2,2}(3 \triangle t)+\triangle_{2} q_{2} F_{2,2}(2 \triangle t)+\triangle_{3} q_{2} F_{2,2}(\triangle t) \\
+\ldots+q_{n} F_{2, n}(3 \triangle t)+\triangle_{2} q_{n} F_{2, n}(2 \triangle t)+\triangle_{3} q_{n} F_{2, n}(\triangle t)
\end{array}
$$

and for well segment $n$ :

$$
\begin{array}{r}
\triangle p_{n}=q_{1} F_{n, 1}(3 \triangle t)+\triangle_{2} q_{1} F_{n, 1}(2 \triangle t)+\triangle_{3} q_{1} F_{n, 1}(\triangle t) \\
+q_{2} F_{n, 2}(3 \triangle t)+\triangle_{2} q_{2} F_{n, 2}(2 \triangle t)+\triangle_{3} q_{2} F_{n, 2}(\triangle t) \\
+\ldots+q_{n} F_{n, n}(3 \triangle t)+\triangle_{2} q_{n} F_{n, n}(2 \triangle t)+\triangle_{3} q_{n} F_{n, n}(\triangle t)
\end{array}
$$

In the above array of equations, $q_{1}$ is the flow rate from the 1 st segment at $t=\triangle t$; $\triangle_{2} q_{1}=q_{1}(2 \triangle t)-q_{1}(\triangle t)$ and $\triangle_{3} q_{1}=q_{1}(3 \triangle t)-q_{1}(2 \triangle t)$.

The last equation for this set is obtained based on type of well constraint that we have at this time.

Solving the above set of equations, as we did for the first time step, we can solve for the flow rate distribution along all the well divisions at the third time step. This procedure can be repeated for all the time steps.

\subsection{Frictional Pressure Drop in the Wellbore}

Currently we are working on coupling our wellbore friction models to the analytical solution presented above. This would let us analyze the effects of wellbore friction 
on horizontal well productivity under transient conditions. One way of accomplishing the wellbore/reservoir coupling is as follows:

1. At the end of each time step, using the analytical solution developed in the previous section, we calculate the productivity index per unit length of the well $\left(J_{s}\right)$ for each well section.

2. For the first time step, we assume that the pressure in the wellbore is same every where. At the end of this step, we need to evaluate the pressure and flow rate at each of the $\mathrm{n}$ well sections. For this we need $2 \mathrm{n}$ equations.

3. By using an appropriate form of Eq. 4.8 from the previous chapter, we can form $\mathrm{n}$ equations at the $\mathrm{n}$ nodes. By equating Eqs. 4.A2 and 4.A11 from the Appendix of the previous chapter, we can form (n-1) equations that relate the pressure difference between two adjacent nodes to the wellbore pressure drop. For the first time step, the pressure difference between two adjacent nodes will be zero. For the subsequent time steps, the pressure difference between two adjacent nodes will be the pressure difference between these nodes as calculated at the end of previous time step.

4. The final equation required to solve the system of equations is supplied by the well constraint which is either flow rate or BHP specification.

5. The pressure calculated at each node at the end of each time step is kept constant through out the duration of the next time step. They are updated only at the end of the next time step. So this is explicit coupling. At this point we assume that the errors introduced by explicit coupling of the wellbore and the reservoir will be small. We also plan to do implicit coupling so that we can compare the differences between these two methods.

\section{References}

1. Babu, D.K. and Odeh, A.S.: "Productivity of a Horizontal Well," SPE Reservoir Engineering, pp. 417-421, November 1989 


\title{
6. Effects of Grid Systems on Predicting Horizontal Well Productivity
}

\author{
by Jing Wan, Raju Penmatcha, Sepehr Arbabi, and Khalid Aziz
}

\begin{abstract}
This study compares horizontal well performance simulated by a uniform coarse grid, a uniform fine grid and a non-uniform fine grid using the Peaceman well model. An analytical solution is also used to compare with the numerical solutions. We find that when using a coarse grid with conventional well models, the flow rate of the wel 1 is underpredicted, which means the well index calculated by Peaceman formula needs to be increased for the numerical simulator to predict the well productivity correctly. Our analysis shows that the explicit modeling of wells, as done in a recent paper, can lead to incorrect results.
\end{abstract}

\subsection{Introduction}

When solving the flow equations of a reservoir numerically, the block pressure cannot be assumed to be equal to the wellbore pressure. These two pressures can be related by a numerical, semi-analytical or analytical well model, which gives the appropriate well index. Peaceman [1] has derived expressions for well index under the condition of 2D flow from an isolated well under steady state or pseudo-steady state condition. Babu and Odeh [2, 3] have considered a more general case and have obtained an analytical solution for the steady-state or pseudo-steady state case with an arbitrarily located well in the block, provided the well is along one of the grid lines. Penmatcha et al. [4] have extended Babu and Odeh's model to the case of infinite well conductivity, which yields the expected non-uniform flux pattern along the wellbore.

Most current commercial simulators use the Peaceman model in spite of its limitations. Dietrich and Kuo [5] have compared horizontal well performance simulated by a uniform coarse grid with a non-uniform fine grid system using the Peaceman model. They concluded that the well productivity is overpredicted using a coarse grid and the conventional well model proposed by Peaceman. Dietrich and Kuo also applied an explicit modeling technique using a non-uniform grid system, in which the grid was refined around the well to represent the length and the diameter of the horizontal well. They also set the permeability of the wellbore gridblocks at a very high level to establish a negligible pressure gradient along the wellbore. At the same time, a very large well transmissibility connection factor was used for the wellbore at the heel to force the well and the well block pressures to be the same, thus deactivating the well model.

The numerical and analytical solutions presented here show that for the case considered by Dietrich and kuo, well productivity is underpredicted using a coarse grid system with a conventional well index, which is different from the results of Dietrich and Kuo. We also show that the reservoir performance simulated by a non-uniform fine grid can deviate significantly from that simulated by a uniform fine grid and that calculated by the appropriate analytical well model. 


\subsection{Well Model}

The well index (WI), which accounts for the geometric characteristics of the well and the reservoir properties around the well, is used to relate the well bottomhole pressure and the well block pressure.

\subsubsection{Peaceman Formula}

Assuming single phase flow we can relate well and well block pressures through the radial form of Darcy's law:

$$
\begin{gathered}
q_{p}=W I \frac{k_{r p}}{\mu_{p} B_{p}}\left(P_{p, \text { block }}-P_{\text {well }}\right) \\
W I=\frac{\theta k h}{\ln \left(\frac{r_{0}}{r_{w}}\right)+S}
\end{gathered}
$$

where $\theta$ is the angle open to flow and $\mathrm{S}$ is the skin factor.

Peaceman [1] derived the following expression for $r_{0}$ (for a well parallel to the $\mathrm{x}$-axis):

$$
r_{0}=0.28 \frac{\left[\left(k_{z} / k_{y}\right)^{1 / 2} \Delta y^{2}+\left(k_{y} / k_{z}\right)^{1 / 2} \Delta z^{2}\right]^{1 / 2}}{\left(k_{z} / k_{y}\right)^{1 / 4}+\left(k_{y} / k_{z}\right)^{1 / 4}}
$$

It is based on the assumptions of single phase flow, homogeneous reservoir, uniform grid, uniform permeability, an isolated well (the well is at least five gridblocks away from all boundaries and ten gridblocks away from other wells [6]), pseudo-steady state or steady state conditions, and block-centered well. Since a horizontal well cannot be far from the top or bottom boundary when the reservoir is thin, the accuracy of Peaceman's well model is restricted. Furthermore the flow around a horizontal well may not be two dimensional.

\subsubsection{Babu and Odeh's Analytical Solution}

Babu and Odeh [2] considered a more general case, where the well can be arbitrarily located at any position in a block, provided that it is parallel to one of the axes or gridlines. This analytical solution is based on steady-state (or pseudo-steady state)

However, Babu's analytical solution is based on the assumption of uniform flux along the wellbore. In reality, the flux pattern along the well is non-uniform, which is also the case for the problem considered by Dietrich and Kuo [5]. Recently, Penmatcha at al. [4] have developed a solution for a well with non-uniform influx and infinite conductivity. The wellbore is assumed to have a uniform pressure, resulting in non-uniform flux along the well length. Later we will use this analytical solution to evaluate the adequacy of numerically computed well productivity.

\subsection{Grid Systems}

\subsubsection{Uniform Grids}

In the previous section, we discussed the fact that the Peaceman well model is based on a uniform grid. For homogeneous, uniform permeability, single phase flow reservoir simulation, it is appropriate to use this model. 
In practice, the limitation of computational resources requires that the grid be as coarse as possible with refinement in some regions; therefore we need to ensure that the well model is correct. All well models are based on some assumptions, so the model used needs to be appropriate for the problem being considered. We will show in the next section how this is accomplished.

\subsubsection{Non-uniform Grids}

The use of non-uniform grid spacing is often required in reservoir simulation. For example, it is necessary for 3-D simulations of stratified reservoirs or for well coning calculations [5]. Dietrich and Kuo [5] used the approach of grid refinement and deactivating the wellbore to realize explicit well modeling. In this approach, the wellbore is represented by a series of very small gridblocks scaled to represent the length and diameter of the horizontal well, the permeability of the wellbore is set at a high level and the transmissibility connection factor for the wellbore at the heel is also made very large, so that the well model itself can be removed from the problem and the effect of the well index is trivial. Then they assumed that this scheme will yield accurate solutions for horizontal well productivity.

However, we have found that this approach has two significant disadvantages:

1. Numerical Approximation:

Aziz and Settari [6] showed some disadvantages of irregular grids considering the accuracy of finite-difference approximation. In most of current commercial simulators, the block-centered grids are used. Aziz and Settari have shown that this type of grid causes higher numerical errors than point-distributed grids. In the example below, we will show the divergence of the results by a non-uniform fine grid from both a uniform fine grid and an analytical solution.

2. Physical Restrictions:

Palagi [7] discussed well models for Cartesian and Voronoi grids. The numerical results of block pressure obtained with large grid aspect ratios do not agree well with analytical solutions for locations close to the wells. In spite of this, the Peaceman's model provides the correct well pressure. The discrepancy of the pressure of gridblocks adjacent to the well is due to the assumption of linear flow used to derive geometric factors for grid connections in the Cartesian system, even in areas of predominantly radial flow like regions around the well. It is clear that this effect is more significant for large aspect ratios.

Dietrich and Kuo's approach of using explicit well modeling actually uses the block pressure as the well pressure. This is possible because by explicit well modeling and the approach to remove the wellbore, the block pressure and the well pressure are the same. However, without the correct well index, and because the block pressure for large aspect ratio grid system will deviate a lot from the correct one, large errors are introduced. 


\subsection{Example Problem}

We tried one example to test the effects of grid systems on predicting horizontal well productivity.

\subsubsection{Model Description}

Table 6.1: Reservoir and Fluid Properties

\begin{tabular}{ll}
\hline$K_{x}, K_{y}$ & $=100 \mathrm{mD}$ (isotropic) \\
$K_{z}$ & $=100 \mathrm{mD}$ (isotropic) \\
$K_{x}, K_{y}$ & $=150 \mathrm{mD}$ (anisotropic) \\
$K_{z}$ & $=5 \mathrm{mD}$ (anisotropic) \\
$\Phi$ & $=0.2$ \\
$C_{t}$ & $=3.0 \times 10^{-5} \mathrm{psi}^{-1}$ at $P_{\text {ini }}$ \\
$q_{\max }$ & $=10000 \mathrm{STB} / \mathrm{d}$ \\
$b h p_{\min }$ & $=2001.521 \mathrm{psia}$ \\
$\mu$ & $=1 \mathrm{cP}$ \\
$\mathrm{B}$ & $=1 \mathrm{RB} / \mathrm{STB}$ \\
$P_{\text {ini }}$ & $=3596.94 \mathrm{psi}$ \\
Drainage area & $=2640 * 2640 \mathrm{ft}^{2}$ \\
Thickness & $=100 \mathrm{ft}$ \\
$L_{w}$ & $=1000 \mathrm{ft}$ \\
$\mathrm{S}$ & $=0.0$ \\
$r_{w}$ & $=0.1875 \mathrm{ft}$ \\
\hline
\end{tabular}

We used a three-dimensional, homogeneous closed reservoir. A single horizontal producer is centered in the reservoir and partially penetrates the reservoir in the y-direction. We assume only oil phase exists in the reservoir, so it is a single phase problem. Both constant bottomhole pressure and constant flow rate constraints are used at the horizontal well. Horizontal well production rates are all evaluated under pseudo-steady state when the production rate is constrained. But when the bottomhole pressure is constrained, it is not possible to reach pseudo-steady state, we will elaborate further on this point later.

The properties and controlling parameters in simulation are shown in Table 6.1. The configuration of the non-uniform fine grid is exactly the same as that used in reference [5].

\subsubsection{Analysis of Results}

All the numerical results were obtained using a homogeneous single phase flow model in the Eclipse reservoir simulator. The analytical solution is used as the yardstick to measure the adequacy of simulation results.

Constant Bottomhole Pressure Constraint on the Well

(i) Results by the uniform fine grid system and the analytical solution 
Figure 6.1 shows that simulations with the coarse grid underpredict well productivity over most of the simulated period. Figure 6.2 depicts an expanded region of Figure 6.1 from 20 to 25 days to better show differences among various results. Figure 6.3 is a typical plot used in well testing. We find that there is no unit slope line, which means pseudo-steady state was never reached in this case. Nevertheless, we will use this case to compare our results with those of Dietrich and Kuo's. We can see from Figures 6.1 and 6.2 that the curve of the uniform fine grid is closest to that of the analytical solution. In this case, the Grid Aspect Ratio (GAR) is equal to 6. It should be mentioned that simulations were done with no gravity in order to be consistent with the currently available analytical solution.

Figure 6.4 shows the flow rate ratio vs. well index ratio. $W I_{0}$ is the default well index value calculated by Eclipse for the coarse grid simulation, and increased WI is obtained by multiplying it by a factor. The flow rate ratio is the ratio of the coarse to uniform fine grid or coarse to analytical solution. We note that the Peaceman's well index, which is the default in Eclipse, has to be multiplied by a factor corresponding to the flow rate ratio of 1 to yield the correct coarse grid solution. In this case, the correct well index ratio is flow rate dependent and varies from 1.24 to 1.44. In Figure 6.4, we observe two different trends, one is when the well index ratio increases with increasing oil rate ratios, which corresponds to the period that the pressure derivative curve has a negative slope; the other is that when the well index ratio increases while the oil rate ratio decreases, it corresponds to the period when the derivative curve has a positive slope.

\section{(ii) Different Schemes to Obtain Irregular Grids and Explicit Modeling Results}

Figures 6.1 and 6.3 show the production rate of the horizontal well calculated by different grids and well models. There are three curves referring to the flow rates simulated by non-uniform fine grids. By setting high permeability to the wellbore and high WI to the heel, we can get slightly better results than those from the non-uniform fine grid with no adjustment of $\mathrm{K}$ or WI. While these results are close to those simulated by coarse grids at late times, they are still far from those of uniform fine grids and analytical solutions.

Dietrich and Kuo also evaluated coarse grid simulation by this method, the difference is that they used explicit modeling plus a non-uniform fine grid for their reference solution, which we have demonstrated to be wrong. One reason is that the Grid Aspect Ratio for their problem is about 66 . It is large enough to cause a significant error in the pressure of grid blocks near the well. So their result that the coarse grid overpredicts well productivity is completely different from ours, since we see that coarse grid simulation underpredicts well productivity.

\section{Constant Flow Rate Constraint on the Well}

In this case, the slope of the pressure derivative curve is exactly unity (Figure 6.5), so we can identify the period after about 10 days as pseudo-steady state. Figures 6.6 and 6.7 show that the coarse grid simulation underpredicts productivity at the end of the pseudosteady state period. Again to better show details in results, an expanded region of Figure 6.7 is displayed in Figure 6.8. Figure 6.9 shows that in coarse grid simulation, to obtain the correct horizontal well productivity, the well index should be multiplied by a factor of 1.25 for the isotropic case and by a factor of 1.07 for the anisotropic case. Figure 6.10 demonstrates similar results by the use of bottomhole pressure ratio. 


\subsection{Conclusions}

An explicit modeling procedure employing high resolution grid systems near the well has been shown to be even worse than the coarse grid simulation results. Only when using the approach to deactivate the well model, are reasonable results are obtained, but they are still far from the correct solutions. Numerical approximations and physical restrictions are responsible for this deviation.

Horizontal well rates computed using a uniform coarse grid system and a conventional well model will under-predict well productivity for a well of the type considered.

The correct well index to be used in coarse grid systems can be computed and it is problem dependent.

\subsection{Nomenclature}

Q Flow rate, STB/day

B Formation volume factor, bbl/STB

$k_{r} \quad$ Relative permeability

$k_{x} \quad$ Permeability in the $\mathrm{x}$-direction, $\mathrm{mD}$

$k_{y} \quad$ Permeability in the $\mathrm{y}$-direction, $\mathrm{mD}$

$k_{z} \quad$ Permeability in the z-direction, $\mathrm{mD}$

$r_{w} \quad$ Wellbore radius, $\mathrm{ft}$

$r_{0} \quad$ Effective well radius, $\mathrm{ft}$

$\mathrm{S}$ Skin factor

h Thickness, ft

$C_{t} \quad$ Total compressibility, psi $^{-1}$

$\Delta x \quad$ Gridblock size in the $\mathrm{x}$-direction, $\mathrm{ft}$

$\Delta y \quad$ Gridblock size in the $\mathrm{y}$-direction, $\mathrm{ft}$

$\Delta z \quad$ Gridblock size in the z-direction, $\mathrm{ft}$

$\Phi \quad$ Porosity

bhp Bottomhole pressure, psi

$\mathrm{P} \quad$ Pressure, psi

$P_{\text {ini }}$ Initial pressure, psi

$L_{\text {well }}$ Well length, $\mathrm{ft}$

$\mu$ Viscosity, cP

\section{Subscripts}

p Phase

w Well 


\section{References}

1. Peaceman, D.W.: "Representation of a Horizontal Well in Numerical Simulation," paper SPE 21217 presented at the 11th SPE Symposium on Reservoir Simulation, Anaheim, California, February 17-20, 1991.

2. Babu, D.K. and Odeh, A.S.: "Productivity of a Horizontal Well," SPE Reservoir Engineering, pp. 417-421, November 1989.

3. Babu, D.K. and Odeh, A.S.: "Productivity of a Horizontal Well: Appendices A and B" paper SPE 18334 presented at the 63rd Annual Tech. Conf., 1988.

4. Penmatcha, R. V., Babu, D. K., and Aziz, K.: Effects of Wellbore Friction Factor under Transient Conditions, Chapter 5 of this Annual Report, 1997.

5. Dietrich, J.K. and Kuo, S.S.: "Predicting Horizontal Well Productivity," JCPT, February 29, 1996.

6. Aziz, K. and Settari, A. Petroleum Reservoir Simulation, Elsivier Applied Science, New York, p. 75.

7. Palagi, C.L.: Generation and Application of Voronoi Grid to Model Flow In Heterogeneous Reservoirs, Ph.D. Dissertation, Chapter 6, Stanford University, 1992. 


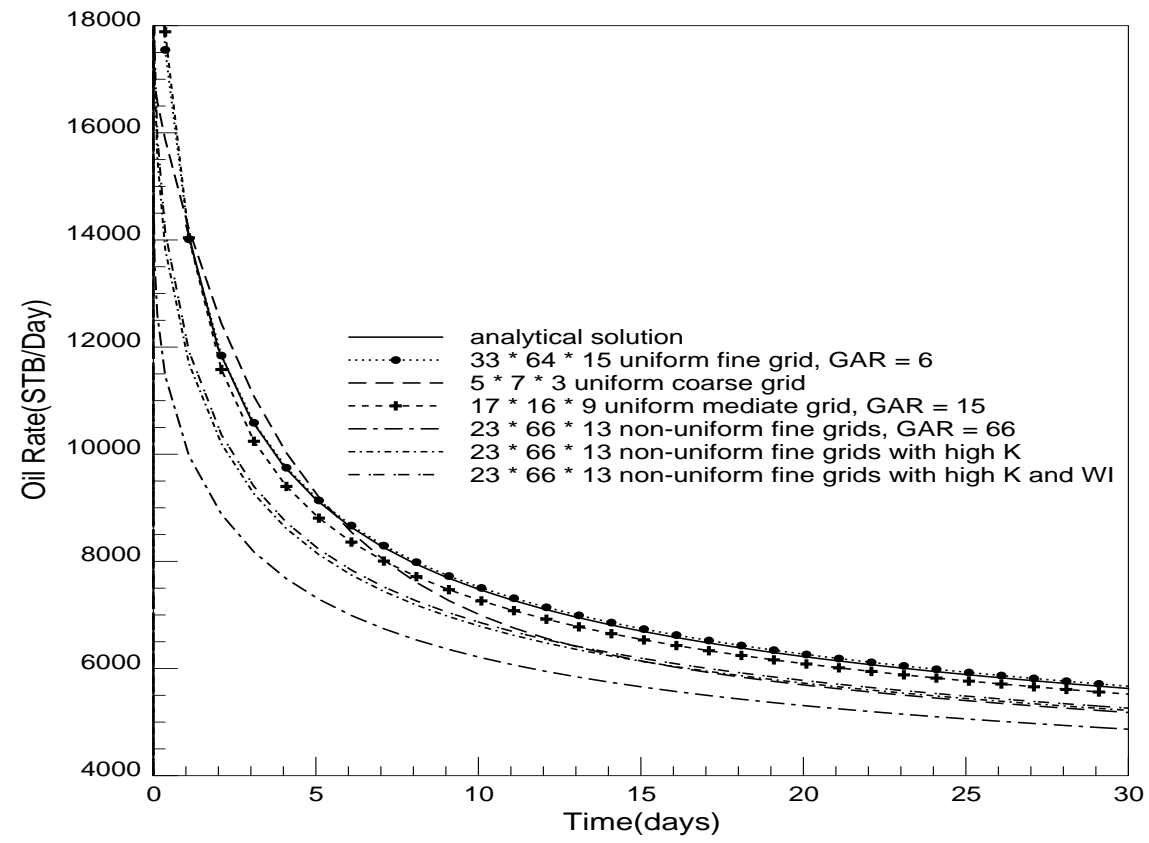

Figure 6.1: Influence of Grid System on Production Ratio for the Case of Constant Well Pressure Constraint

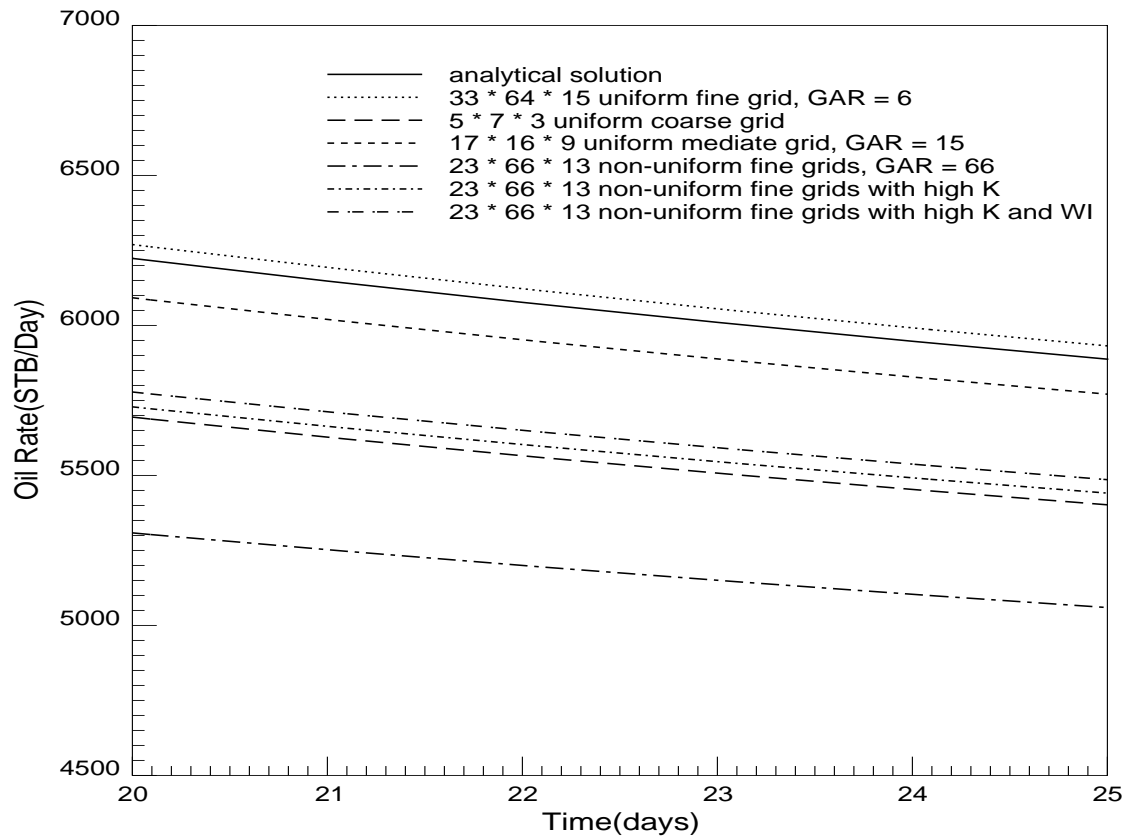

Figure 6.2: An Expanded Portion of Figure 6.1 from 20 to 25 Days 


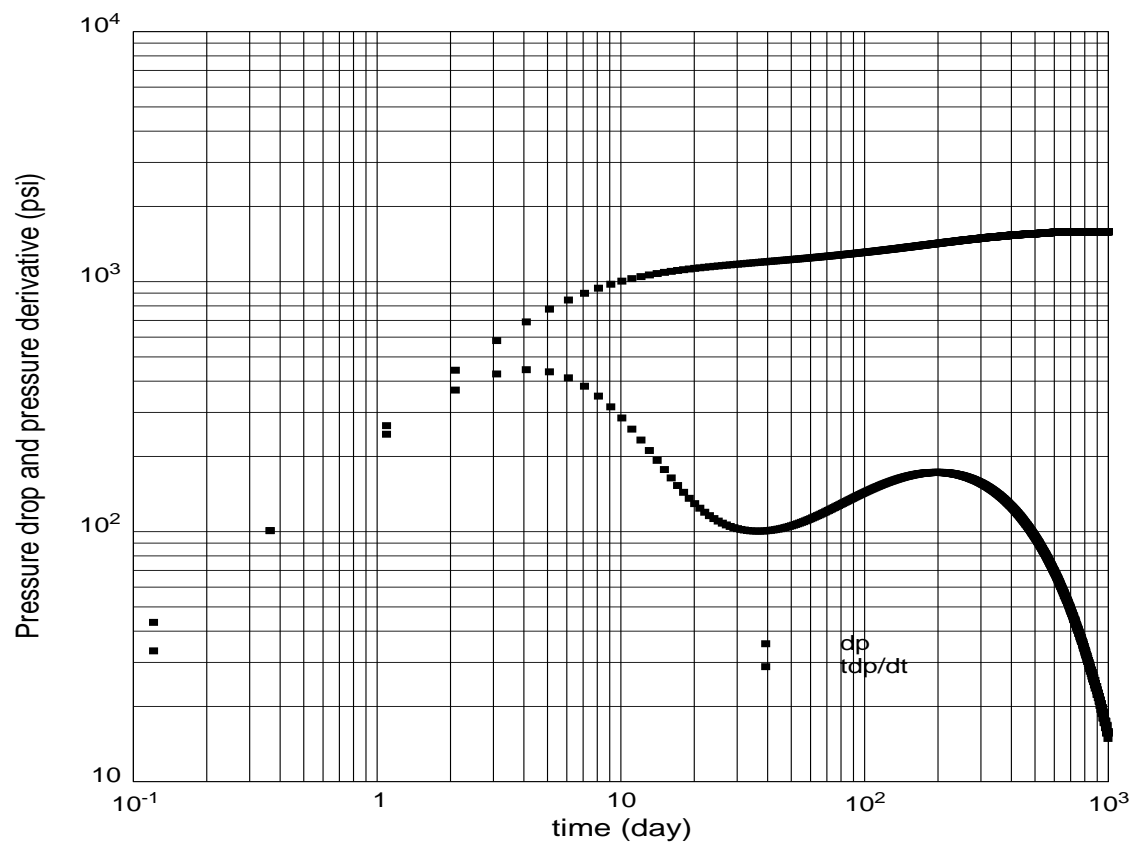

Figure 6.3: Approach to Pseudo Steady State for the Case of Constant Well Pressure Constraint

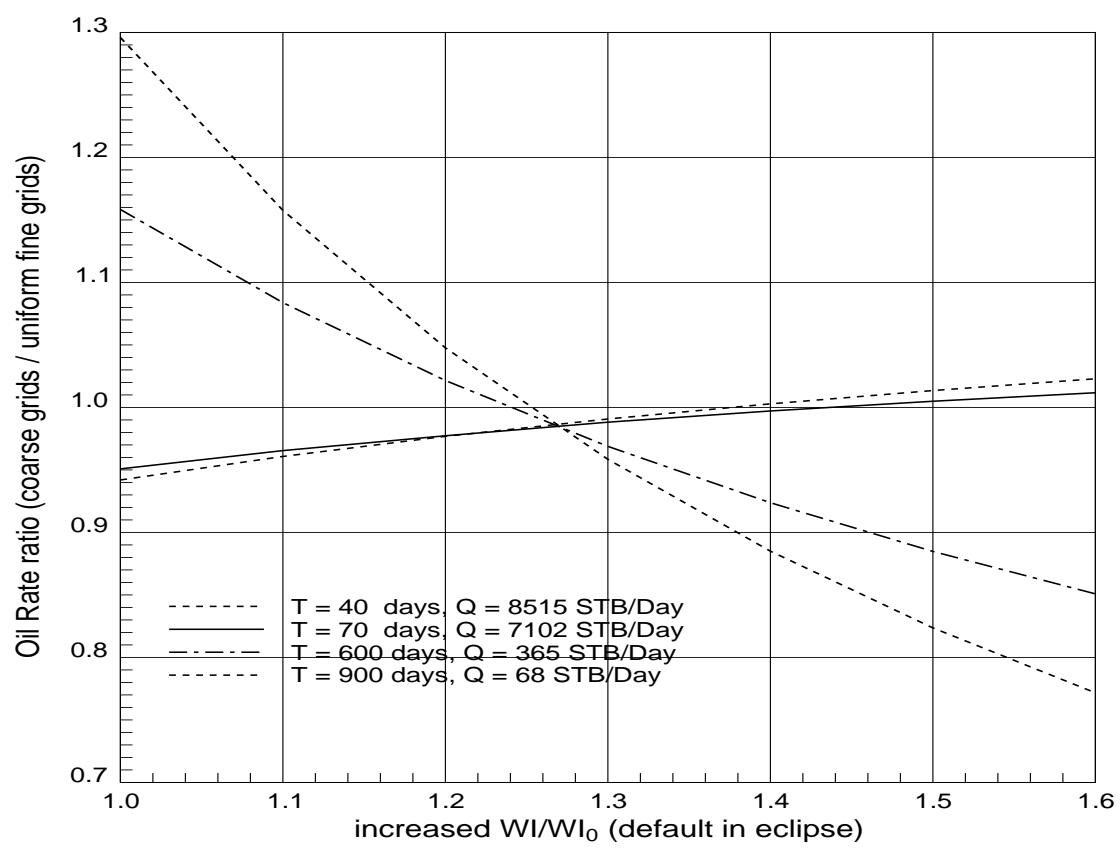

Figure 6.4: Adjustment Factor for WI to obtain Correct Flow Rate - Isotropic Case 


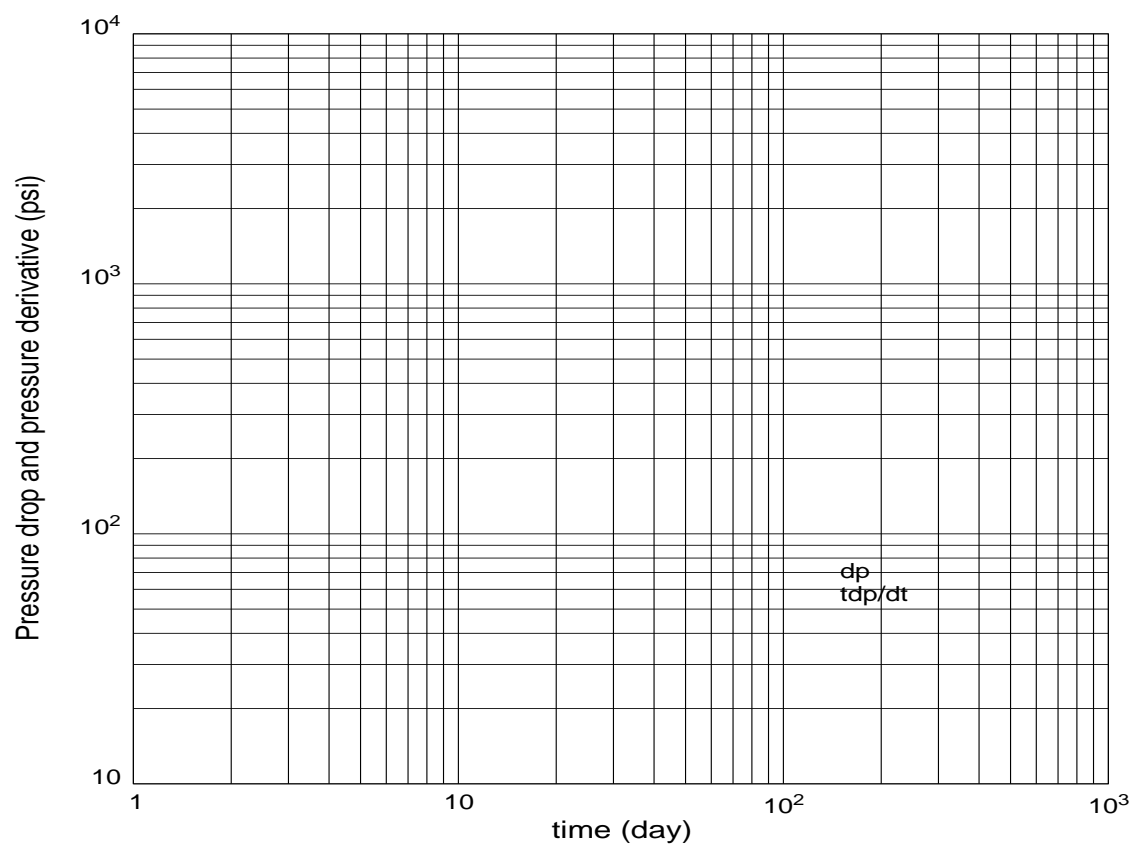

Figure 6.5: Approach to Pseudo Steady State for the Constant Flow Rate Case

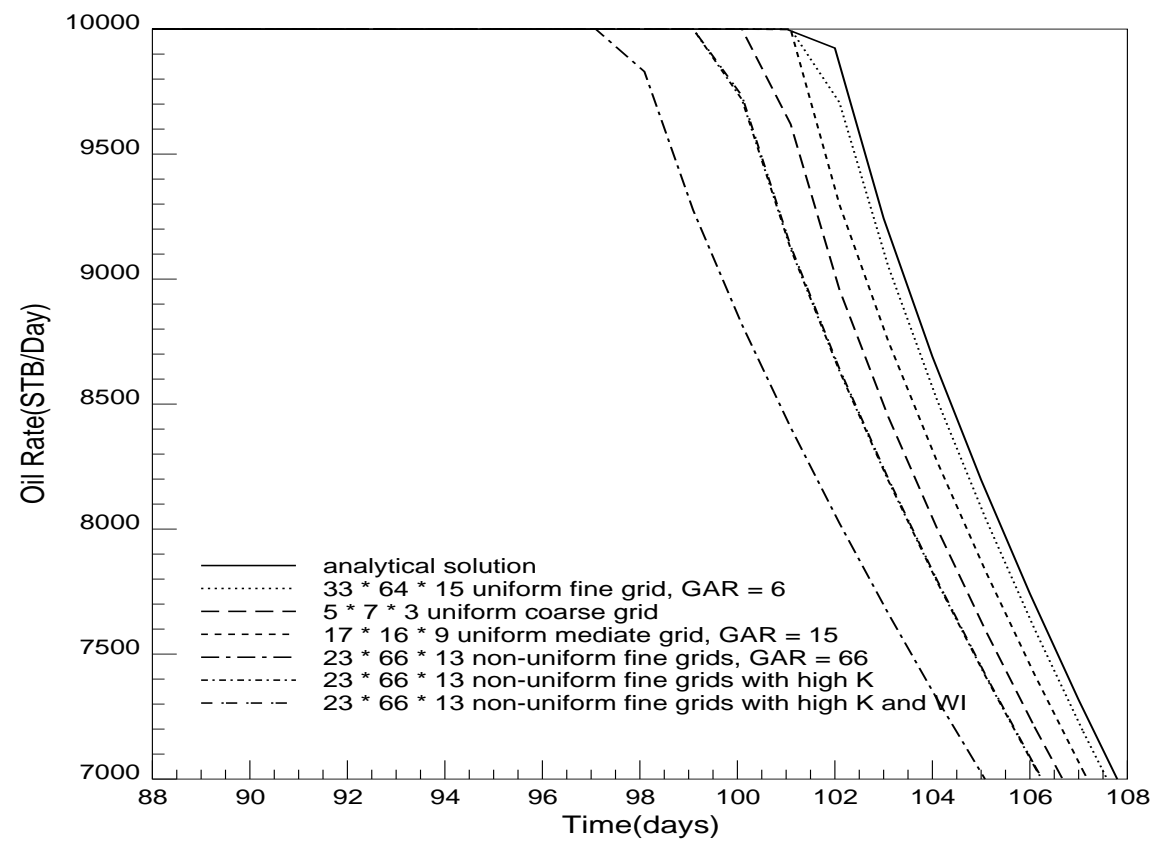

Figure 6.6: Results of Constant Flow Rate Set Initially Followed by a Period of Constant Wellbore Pressure Constraint 


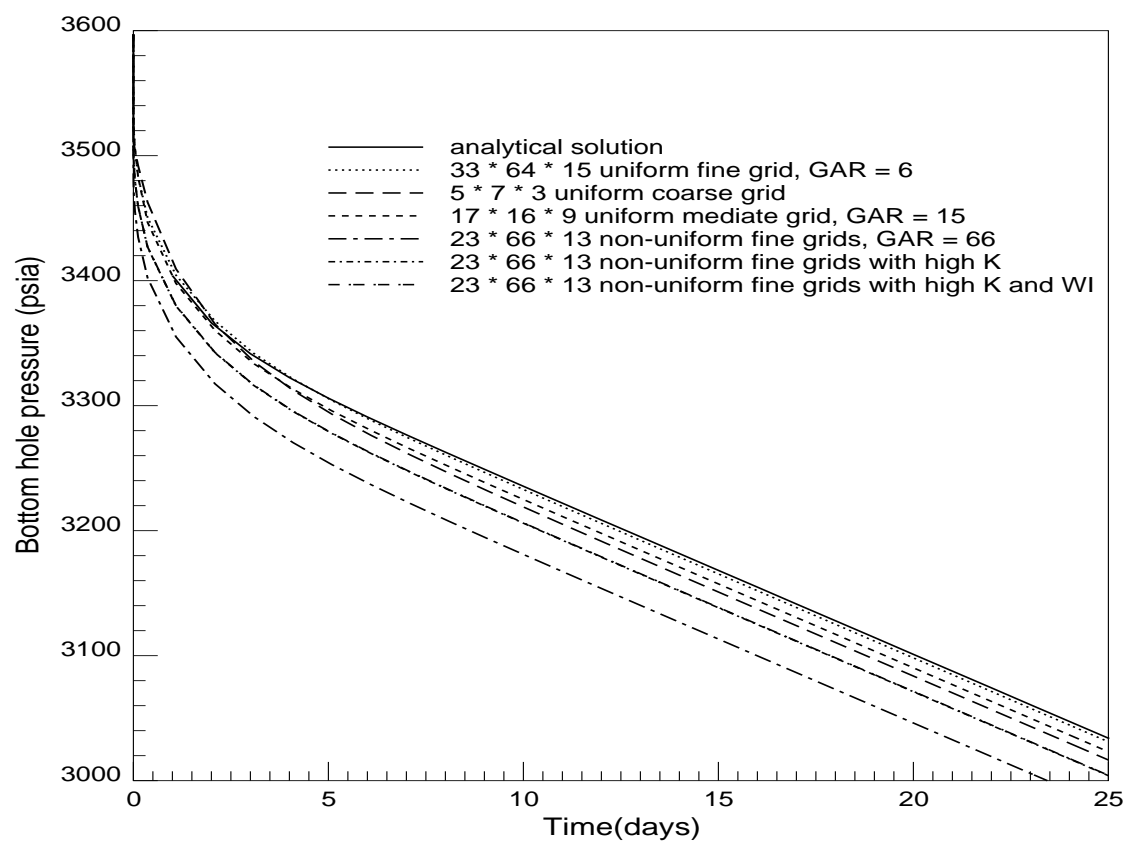

Figure 6.7: Bottomhole Pressure in the Case of Constant Flow Rate Constraint

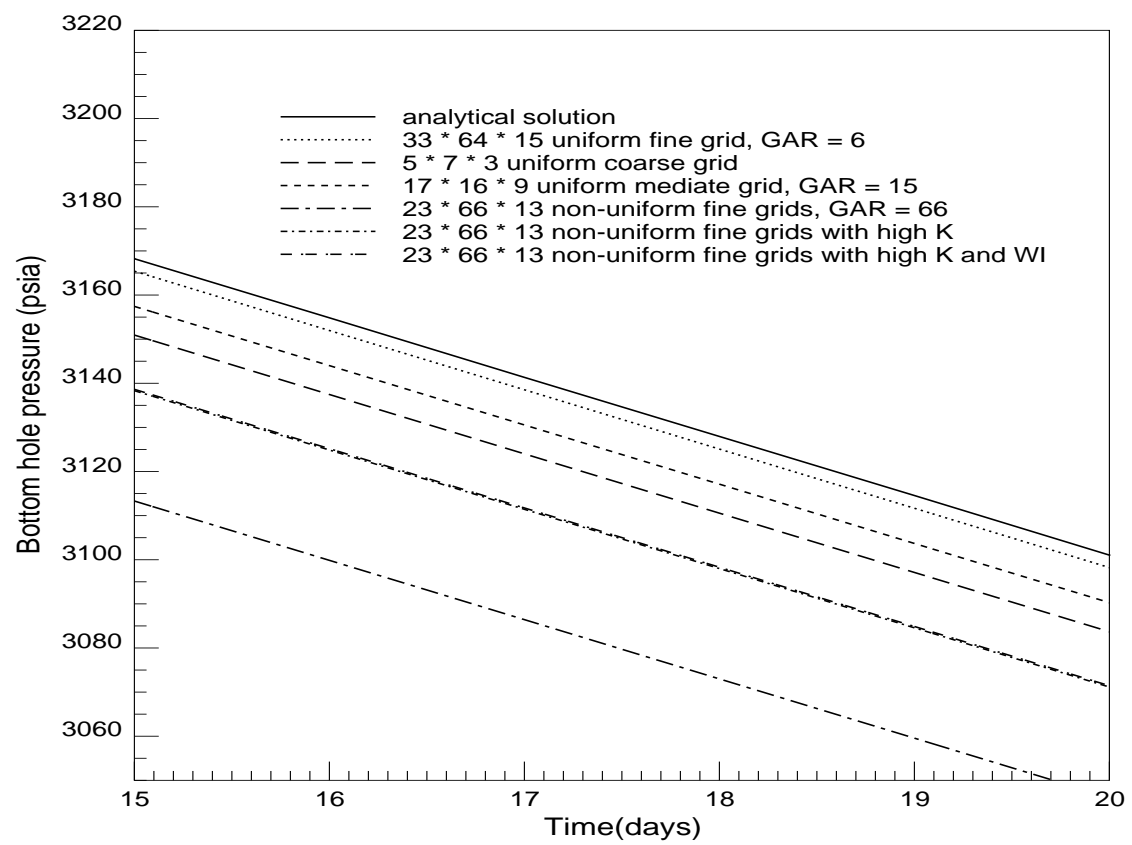

Figure 6.8: An Expanded Portion of Figure 6.7 from 15 to 20 Days 


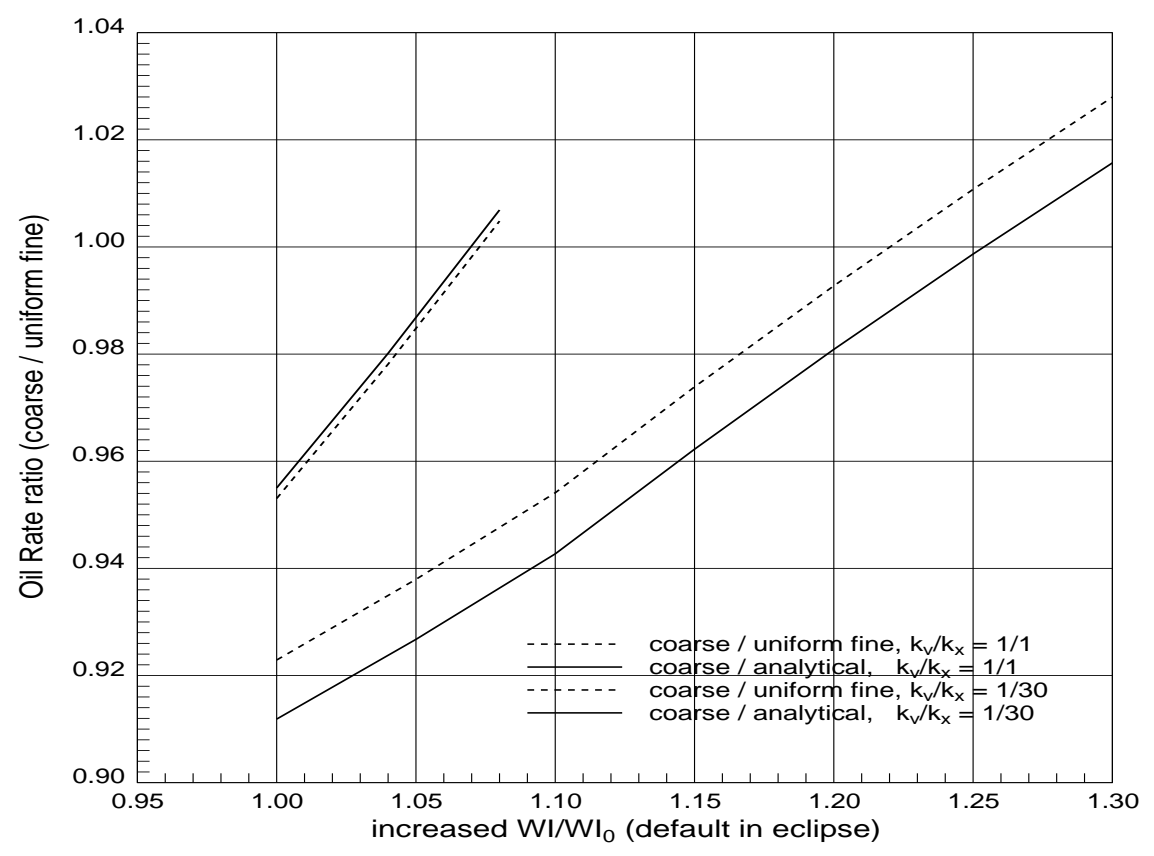

Figure 6.9: Variation of Oil Rate with Well Index for both Isotropic and Anisotropic Cases

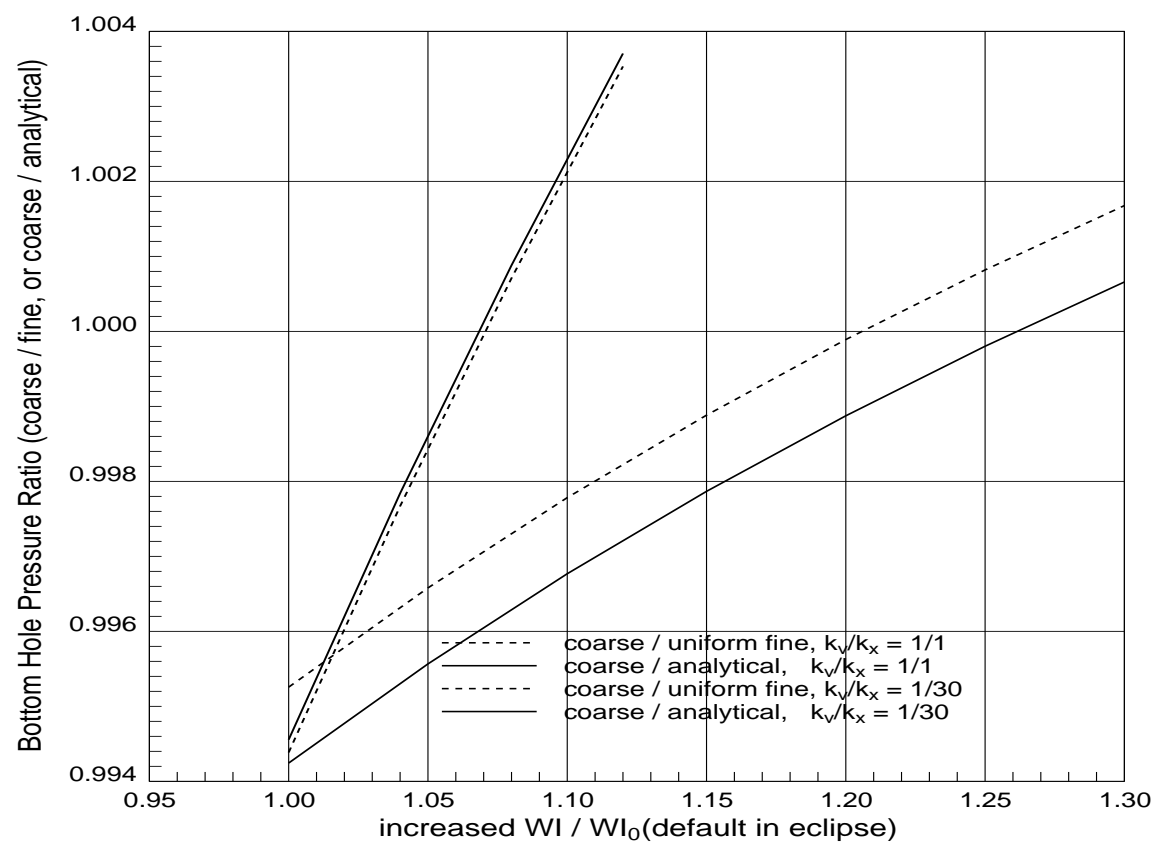

Figure 6.10: Variation of Bottomhole Pressure with Well Index for both Isotropic and Anisotropic Case 


\title{
7. Effect of Reservoir Heterogeneities and other Parameters on Performance Prediction of Non-Conventional Wells
}

\author{
by Khald Aziz, Sepehr Arbabi, and Clayton V. Deutsch
}

\begin{abstract}
Non-conventional wells that include, horizontal, highly deviated and multilateral wells - often called designer wells - are becoming more and more common. Both analytical and numerical tools have been developed and continue to be developed for predicting their performance. Unfortunately, predictions made using these tools rarely match actual performance, except in cases where sufficient production data are available for history matching and the model used for making predictions is selected carefully. Even then the predictions are generally good only for a limited time. In this paper we explore reasons for our inability to make accurate predictions. We consider a case where a vertical well has been drilled and cored. Then, we generate twenty consistent geostatistical descriptions of permeability and porosity that are all constrained to the hard data obtained from the vertical well. Simulations with these realizations show large differences in production rate, WOR and GOR predictions as a result of variations in reservoir properties. It is also shown that the effect of well index (WI) on simulation results is large. Furthermore, for the example considered, analytical models for critical rate and productivity calculations were found to be of limited practical use.
\end{abstract}

\subsection{Introduction}

Wells drilled in petroleum reservoirs interact in a complex way with the reservoir. In order to predict their behavior we must be able to model multiphase flow in the well and the reservoir. In this problem there are many sources of uncertainties, some of these are explored in this paper. The most serious of these is the limited data about the reservoir itself.

As with everything else in nature, in the end the best we can hope to do is to predict the performance of horizontal wells only in a probabilistic sense and reduce the uncertainty to a manageable level. In a fascinating paper Beliveau (1995) ${ }^{1}$ has analyzed the performance of 1,306 horizontal wells from 230 fields around the world. He shows that the productivity improvement factors (PIF) - defined as the initial stable oil or gas production rate of a horizontal well divided by the current production of an offsetting vertical well - have an approximately log-normal distribution because "most reservoir parameters are log-normally distributed about their mean." He shows that horizontal wells in conventional reservoirs have a mode (most likely) PIF of 2, a median of (50/50) 3 and an average (or mean) of 4. In heavy oil reservoirs the mode was about 5 and in fractured reservoir about 6 . Beliveau also compared forecasts of well performance with actual results. Here he found that in a 13-well program in a North Sea field actual results were 14\% higher than initial forecasts; however, the average of the absolute individual well errors was much higher at $43 \%$. Only 8 of the 13 producers had actual results within $\pm 50 \%$ of the forecasts. Furthermore the predicted ranking of wells was not preserved 
during their production phase. Beliveau ${ }^{1}$ also summarizes Shell Canada's experience with 75 horizontal wells in several fields: Actual average results exceeded forecasts by about 20\%; while the average absolute error (error bar) was almost $60 \%$. Fewer than half the wells performed within $\pm 50 \%$ of their forecasts. While Beliveau makes repeated reference to the importance of geological heterogeneity, he does not quantitatively investigate its effect on well performance.

In another study by Mullane et al. (1996) ${ }^{2}$ performance of 29 horizontal wells was compared with predictions. While in this case the estimated oil rate was hit exactly, the average absolute error was $47 \%$. Predictions of only about half the wells were within $\pm 50 \%$ of actual performance. They used a “calibrated" version of Joshi's deliverability equation (Joshi, 1986) ${ }^{3}$ to predict oil rate.

In published field scale evaluations, oil rate at a given drawdown appears to be the most common predicted quantity that is compared with field performance. In this paper we will show how more detailed well performance predictions differ with different geological descriptions constrained to the same data, different grid block sizes, and different methods of upscaling absolute permeability. Here we will consider time for which plateau production can be sustained, WOR and GOR. These factors are much more difficult to predict than fluid rate. In addition we will show the effect of techniques used for modeling wells in simulators. In particular we see the effect of the well index and the pressure drop in the well. Finally we will compare analytical and numerical results for water cresting into a horizontal well and well productivity.

\subsection{What is Needed for Predicting Performance}

Predicting the performance of non-conventional (including horizontal wells) or conventional wells requires that we be able to model transient multiphase flows in systems consisting of one or more connected petroleum reservoirs and several wells, that are produced under specified constraints on pressures or rates imposed on individual wells or groups of wells. In order to predict the performance of a well in such complex systems we must be able to

- describe each reservoir (geometry, permeability and porosity distribution);

- describe each well (profile, completion details and the internal condition of the well ;

- model the artificial lift system used in the field;

- model multiphase, multicomponent flows in heterogeneous porous rocks;

- adequately characterize reservoir fluids, and mixtures of reservoir fluids and any fluids that are injected into the reservoir;

- model changes in fluid properties with changes in pressure and temperature (PVT models);

- model the influx of fluids into wells as result of drawdown along wells;

- model multiphase, multicomponent flow in wells; and

- specify the initial state (saturations and pressures) and the boundary conditions (water influx from aquifers) of each reservoir.

None of the above requirements is ever fully met and yet we must make predictions. Furthermore, three-phase, multicomponent flow modeling requires many of assumptions and simplifications that may not be always justified. The job of the engineer is to understand the sources of uncertainty and make the best possible predictions to provide guidance to management in making decisions to meet overall 
corporate objectives. Assessment of uncertainty associated with predictions can be used to associate risk with each option.

Models of various degrees of sophistication may be available or possible. But the level of sophistication must be balanced with (Aziz 1989, 1995) $)^{4,5}$ :

- available resources,

- available information, and

- the objective of the predictions.

It would be ridiculous to try to model the whole reservoir by modeling the flow in individual pores of a network that represents the reservoir. Yet a pore network model of a small portion of the reservoir may provide very useful information about the average behavior of a network of pores, that is represented in reservoir engineering through absolute and relative permeabilities and porosities. This is why our interest spans models of various kinds and complexity - from models for flow in a single pore to full-field models.

In the remainder of this paper we will show how performance predictions are affected by major sources of uncertainty. We will restrict our discussion to predicting the performance of a single horizontal well. First we will discuss the effect on well performance predictions of reservoir description and how it is used in simulators. Next we will show the effect of uncertainty associated with some of the assumptions made in models used for predicting horizontal well performance.

\subsection{Reservoir Description and Simulation Grid}

It is generally acknowledged that lack of knowledge about reservoir heterogeneity is a major cause of the "error bars" associated with performance predictions. Here we will consider a hypothetical example of a horizontal well that is based on data from a real reservoir. The drainage volume associated with the well is $10,000 \times 5,000 \times 100 \mathrm{ft}$.

We will assume that a single vertical well has been drilled. With information from this well and other sources a stochastic model is constructed to produce multiple permeability and porosity images of the drainage volume.

The synthetic reservoir data was fashioned after a fluvial sandstone reservoir with a $70 \%$ net-to-gross ratio. Sequential indicator simulation was used to construct a sandstone/shale lithofacies model. An indicator variogram with a vertical range of $10 \mathrm{ft}$. and an isotropic horizontal range of $100 \mathrm{ft}$. was considered. This resulted in shales that generally extend over less than $100 \times 100 \times 10 \mathrm{ft}$. Porosity and permeability in the shales was set to 0.1 and $1.0 \mathrm{mD}$. The sandstone porosity model was created by sequential Gaussian simulation with a normal scores variogram with a vertical range of $10 \mathrm{ft}$. and an isotropic horizontal range of 2,500 ft. Sequential Gaussian simulation with the collocated cokriging option in GSLIB (Deutsch and Journel, 1992) ${ }^{6}$ was used for the permeability model. The normal scores of porosity were correlated with permeability with a correlation of 0.7 . The normal scores variogram of permeability had a vertical range of $3.33 \mathrm{ft}$. and an isotropic horizontal range of 1,500 $\mathrm{ft}$. The data representing a vertical well that goes through the heel of the horizontal well was extracted from an initial unconditional geostatistical realization. The lithofacies, porosity and permeability data from this vertical well were honored in all twenty geostatistical models. The geostatistical parameters for all realizations are identical and the images appear to be similar. For the medium grid the mean shale fraction is 0.311 
(std. dev. of 0.007 ), mean porosity 0.261 (std. dev. of 0.003 ), and mean horizontal permeability 430.04 (std. dev. 5.71). The horizontal well is aligned along the $\mathrm{X}$-axis and placed in the middle of the drainage volume.

All stochastic images were constrained to the same permeability and porosity at the location of the vertical well and are created on a $100 \times 50 \times 30$ grid $(150,000$ uniform grid blocks, each block is $100 \times 100 \times 3.33 \mathrm{ft}$ ) which is considered to be the fine grid in this study. For the purposes of simulation we have created two sets of upscaled images of the fine grid: a $20 \times 10 \times 10$ medium grid $(500 \times 500 \times 10 \mathrm{ft}$ blocks), and a $10 \times 5 \times 5$ coarse grid $(1,000 \times 1000 \times 20 \mathrm{ft}$ blocks $)$.

The grid is nearly uniform in all cases. The upscaling from the fine grid to the other two grids was done using two commonly used methods:

- numerical single phase flow matching in each of the three directions for each upscaled block and the fine grid blocks contained in that block (referred to as medium- $f$ and coarse- $f$ ), and

- power law averaging (referred to as medium- $p$ and coarse- $p$ ).

Histograms of horizontal and vertical permeability for all three scales are shown in Figure 7.1. The first of the flow based upscaled realizations was used to estimate the power law averaging exponent for the horizontal and vertical directions. Exponents of 0.71 and 0.02 were obtained for the horizontal and vertical directions for the medium grid, and 0.73 and -0.18 for the coarse grid. This gives a total of 100 images to process (20 fine grid, 40 medium grid and 40 coarse grid).

Figures 7.2 through 7.5 show XZ (vertical permeability) and XY (horizontal permeability) slices going through the horizontal well for four selected realizations. These figures compare fine grid descriptions with corresponding upscaled images for the flow based (medium- $f$ and coarse- $f$ ) and power averaging (medium- $p$ ) methods. These images were selected because they showed extreme behavior during performance predictions.

In addition to the grid blocks generated by this process, a layer of homogeneous grid blocks with huge pore volumes $\left(25 \times 10^{12}\right.$ cubic feet $)$ were added to the top and bottom of the reservoir to simulate a large gas cap and a large aquifer. All other data (also extracted from a real field study) are shown in Table 7.1.

Table 7.1: Summary of Rock and Fluid Properties Used

\begin{tabular}{|l|l|}
\hline Initial pressure, $\mathrm{P}_{\mathrm{i}}$, at GOC & $2294 \mathrm{psia}$ \\
\hline Rock compressibility, at $\mathrm{P}_{\mathrm{i}}$ & $1.2 \mathrm{E}-5 \mathrm{psia}^{-1}$ \\
\hline Oil viscosity & $1.3-1.5 \mathrm{cP}$ \\
\hline Gas viscosity & $0.016-0.020 \mathrm{cP}$ \\
\hline Connate water saturation & 0.07 \\
\hline Critical gas saturation & 0.10 \\
\hline Residual oil sat. to water & 0.25 \\
\hline Residual oil sat. to oil & 0.07 \\
\hline Capillary pressure & None \\
\hline
\end{tabular}

The single 2,000 ft horizontal well nearly located in the middle of the drainage volume is produced at 
a rate of 5,000 barrels per day with a minimum bottom-hole pressure of 1,500 psia. The results for oil production rate, GOR and WOR for the medium- $f$ grid are shown in Figures 7.6 to 7.8. Based on these and other results, a single realization (identified on figures showing results) was selected as the base case. Figure 7.9 compares production rates predicted for this base cases by fine, medium- $f$, medium- $p$, coarse- $f$ and coarse- $p$ grids. Figures 7.10 and 7.11 provide corresponding results for GOR and WOR. Because of the large computational time required, it was not possible to process all of the fine grid images. Results of all of the simulations are summarized in Table 7.2. Here values of maximum, minimum, mean, and spread (maximum-minimum) are presented for:

1. Cumulative oil production during the plateau period (when the oil rate is constant at 5,000 barrels per day),

2. Time for the oil rate to drop to 3,000 barrels per day (40\% of the specified rate),

3. GOR at 6,000 days,

4. WOR at 6,000 days. and

5. Bottomhole pressure at 2,000 days.

We have also performed simulations for the base case at the medium grid by using the upscaled geological description from the corresponding coarse grid. This was done to see the effect of grid block size (discretization errors). The results for this case are referred to as medium-f-c and they are compared with other cases on Figures 7.9 to 7.11 . 

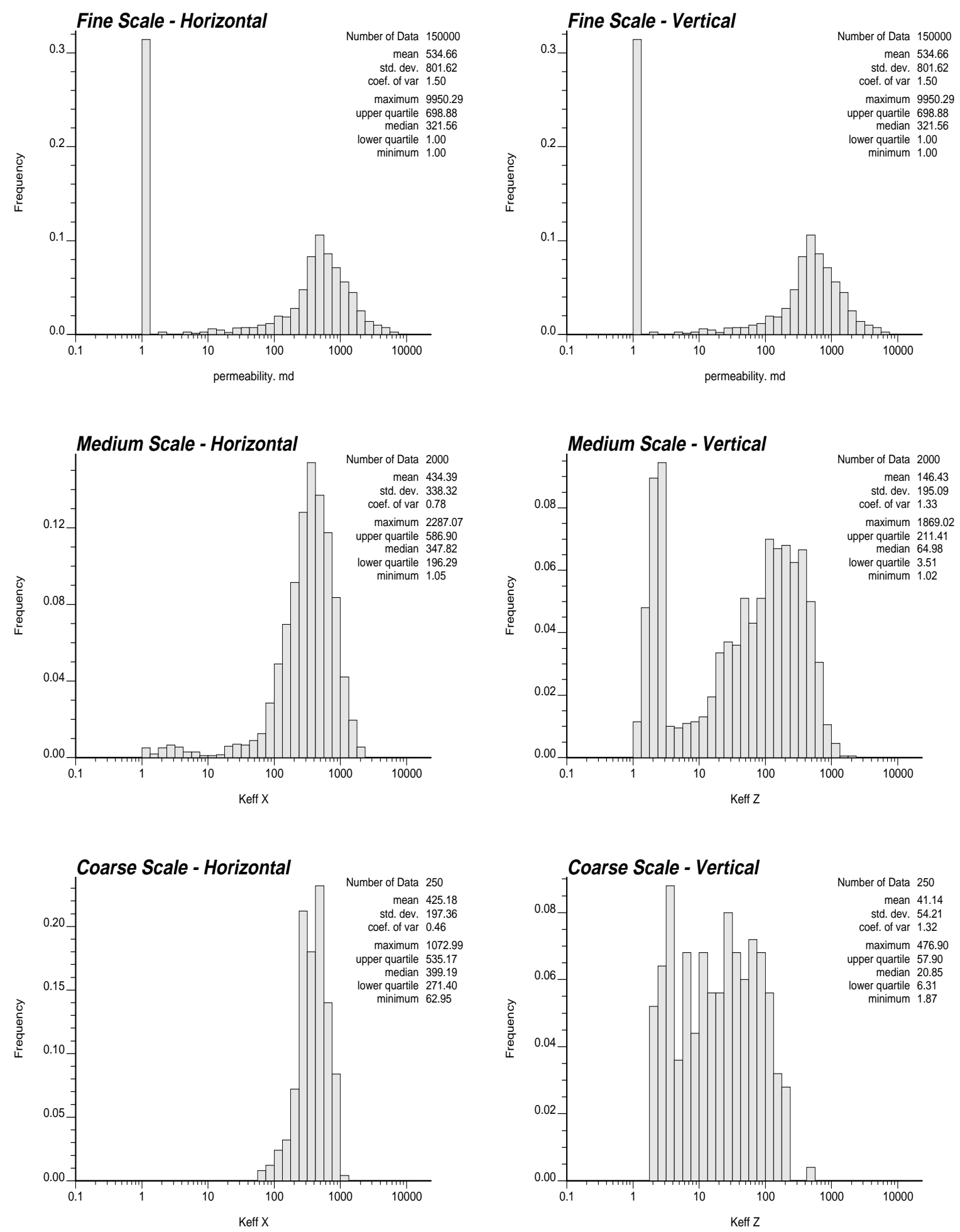

Fig. 7.1: Histograms of horizontal and vertical permeability for fine, medium, and coarse resolutions. A fixed multiplier of 0.1 is applied to the vertical permeability data in the simulations. 

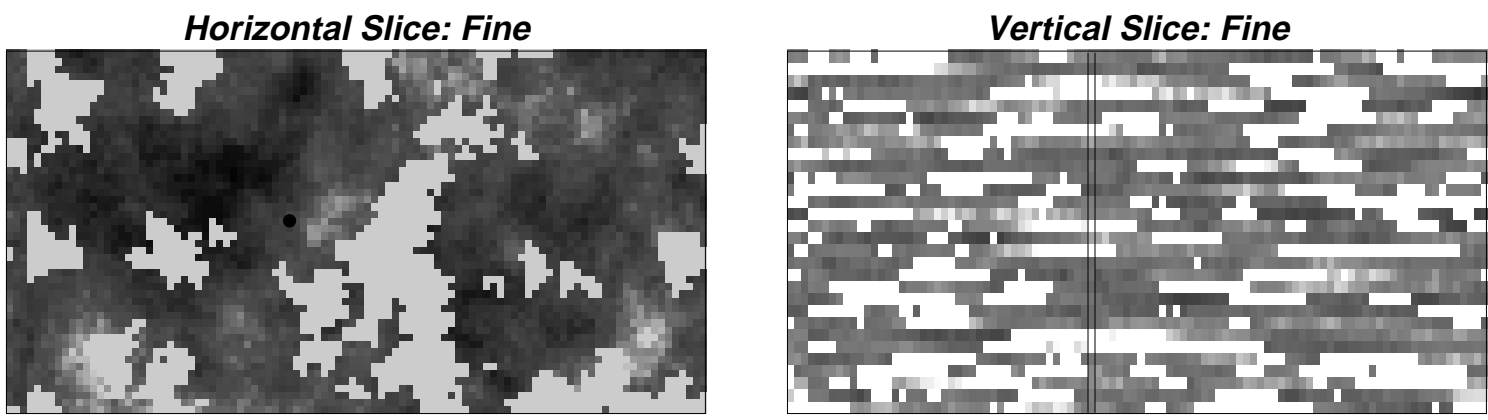

Horizontal Slice: Medium

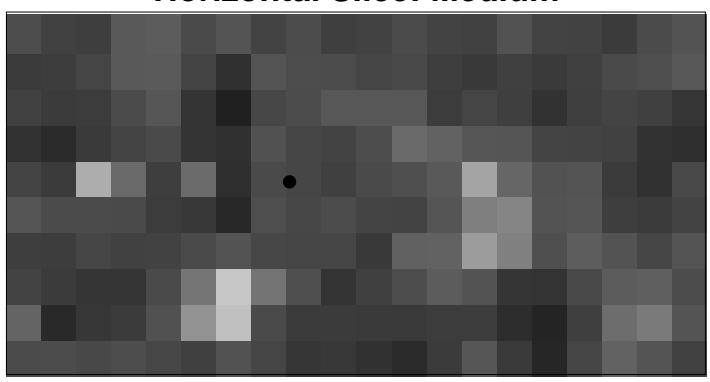

Vertical Slice: Medium

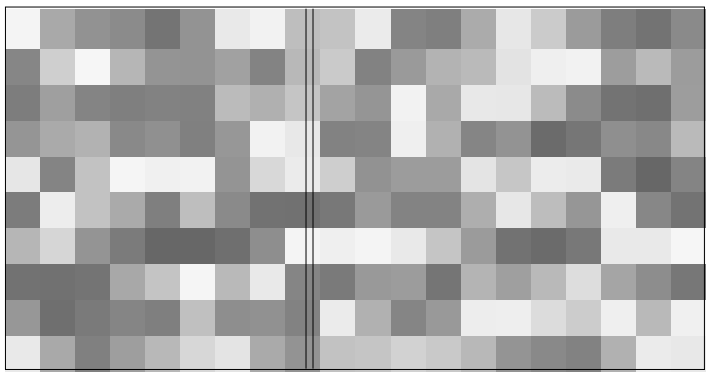

Horizontal Slice: Coarse

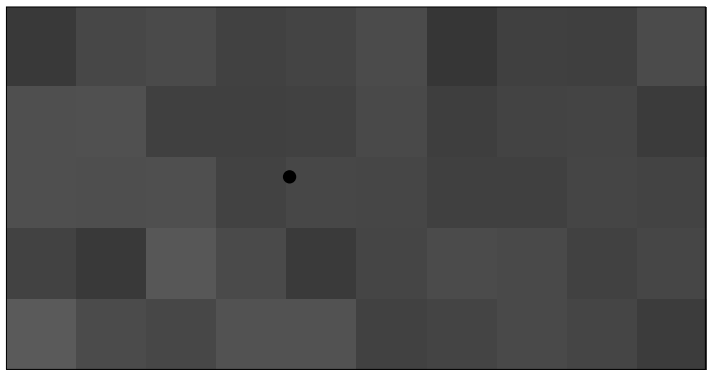

Vertical Slice: Coarse

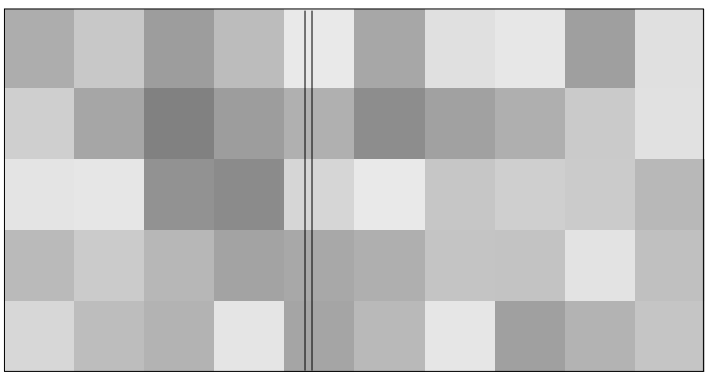

Horizontal Slice: Medium (power average)

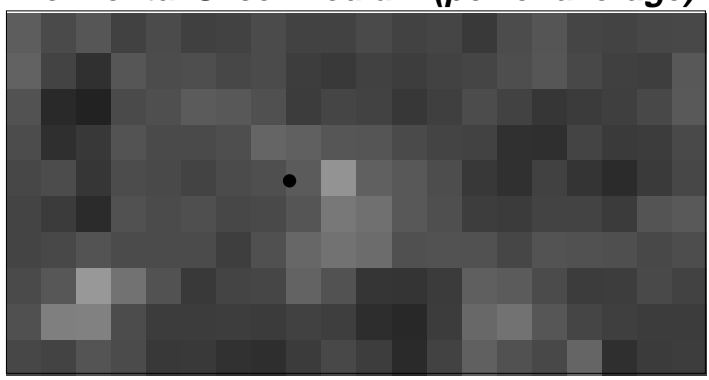

Vertical Slice: Medium (power average)

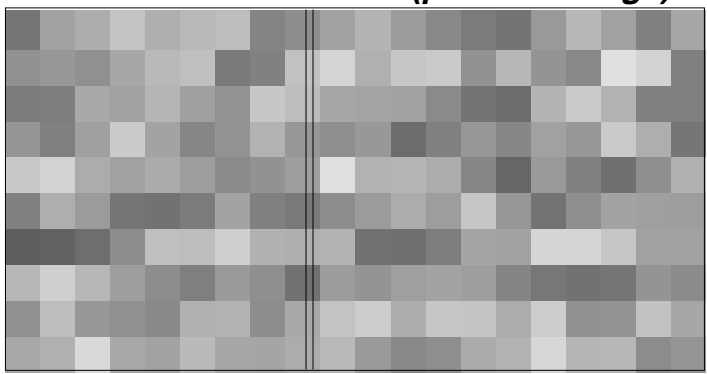

Fig. 7.2: Horizontal and vertical permeability maps of realization 3 for fine, medium-f, coarse- $f$, and medium- $p$ grids. Slices are for the plane of the well. 
Horizontal Slice: Fine

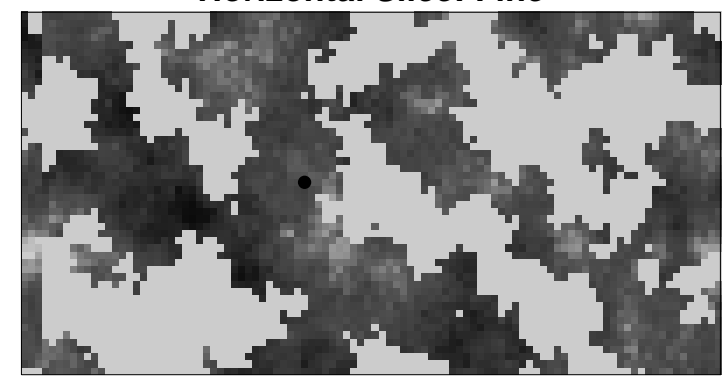

Horizontal Slice: Medium

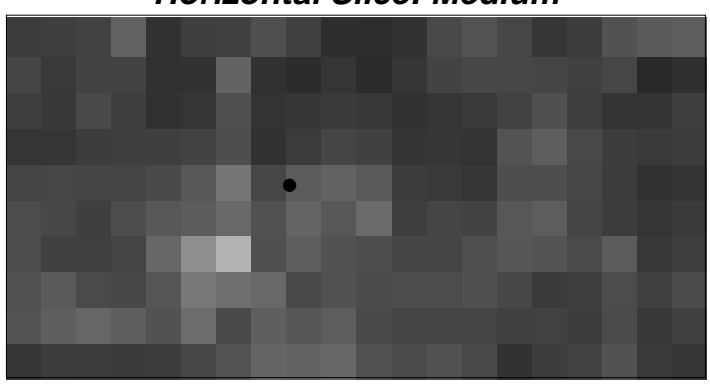

Horizontal Slice: Coarse

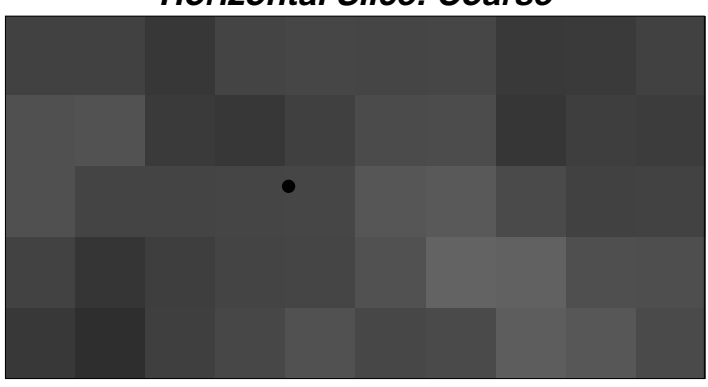

Horizontal Slice: Medium (power average)

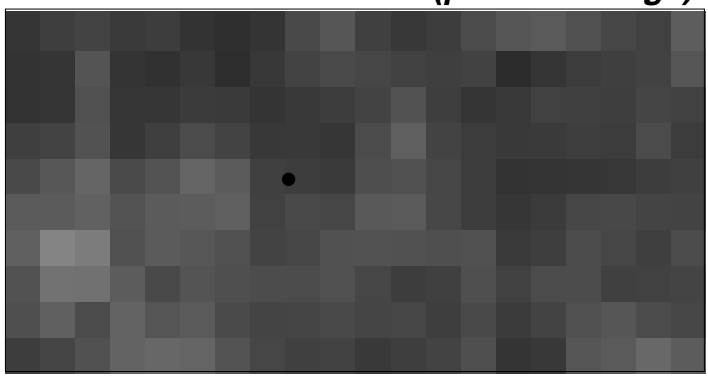

Vertical Slice: Fine

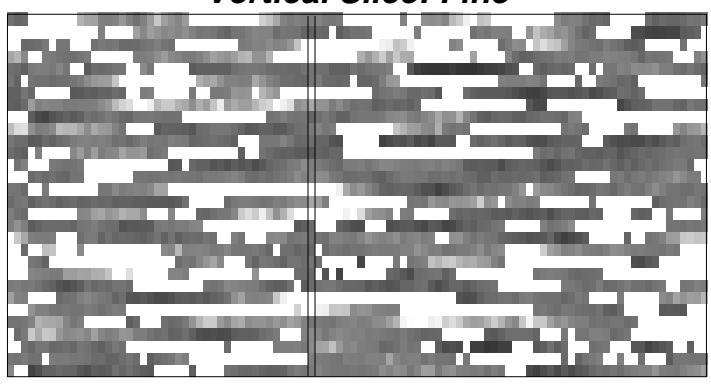

Vertical Slice: Medium

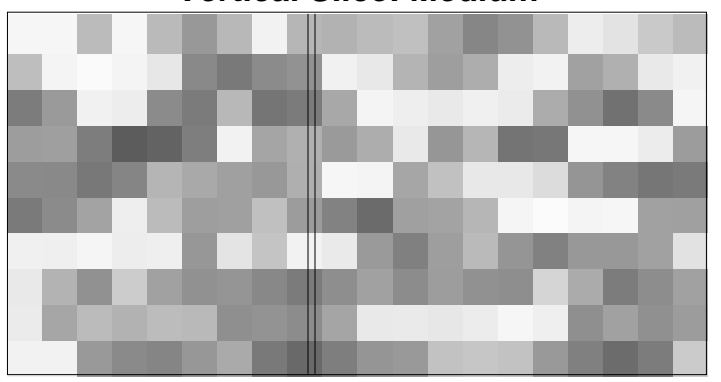

Vertical Slice: Coarse

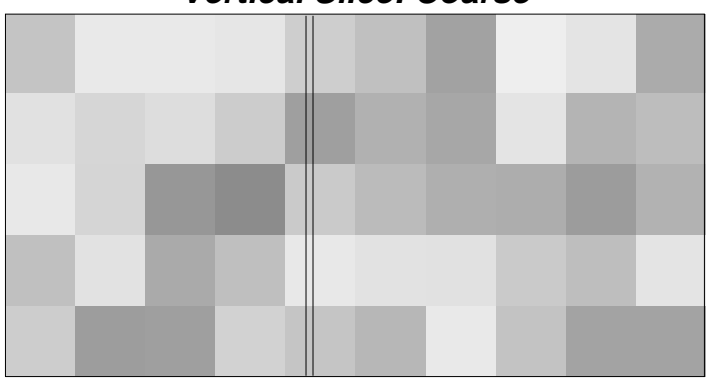

Vertical Slice: Medium (power average)

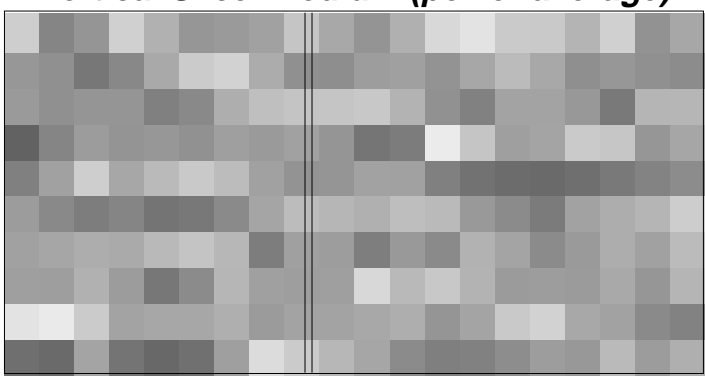

Fig. 7.3: Horizontal and vertical permeability maps of realization 9 for fine, medium-f, coarse- $f$, and medium- $p$ grids. Slices are for the plane of the well. 

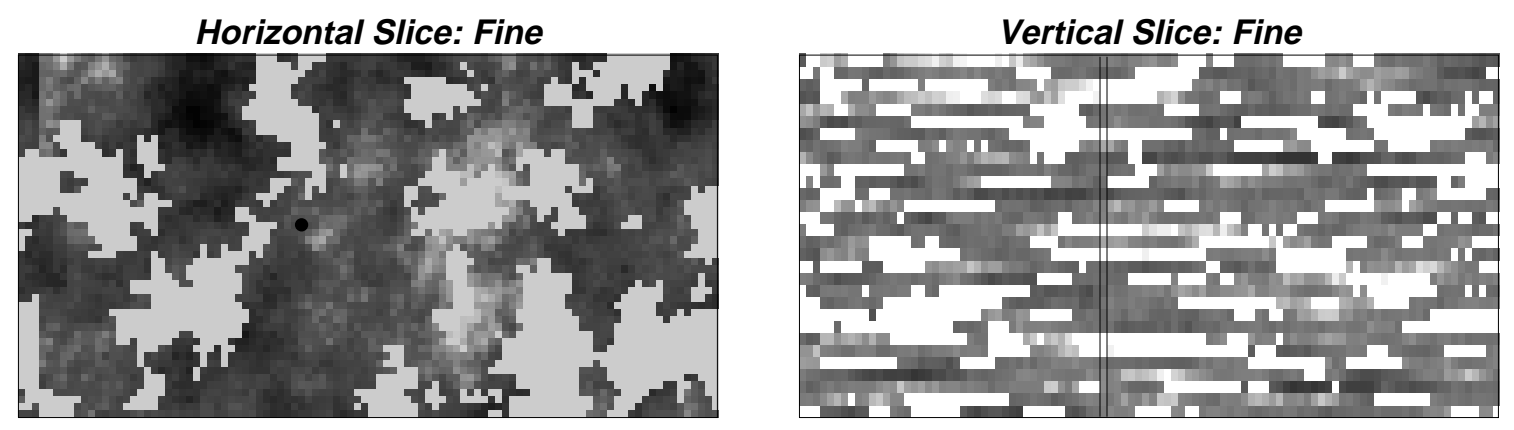

Horizontal Slice: Medium
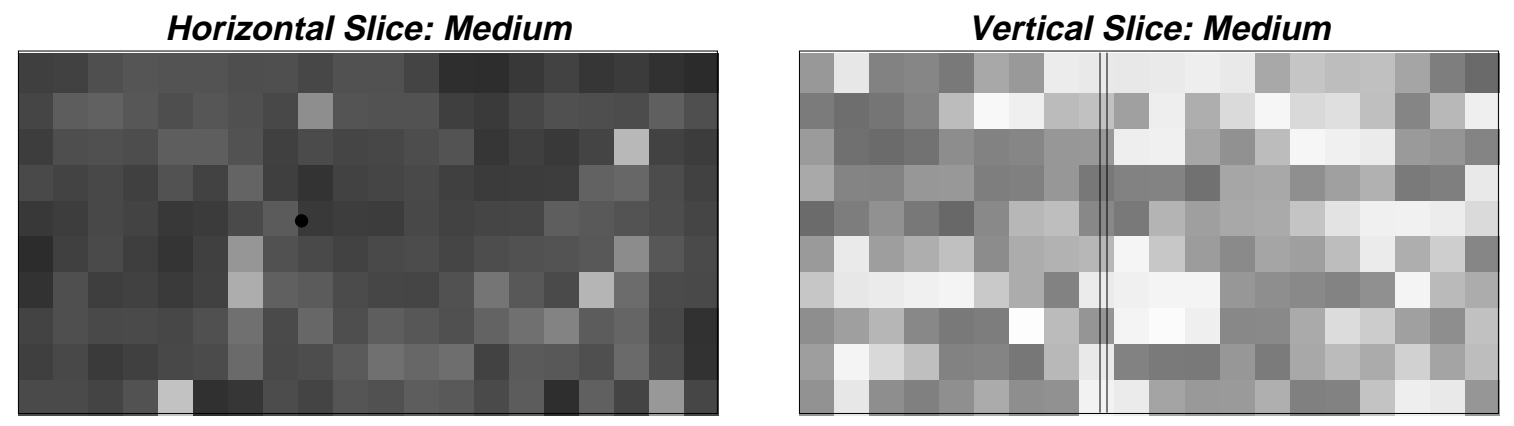

Horizontal Slice: Coarse

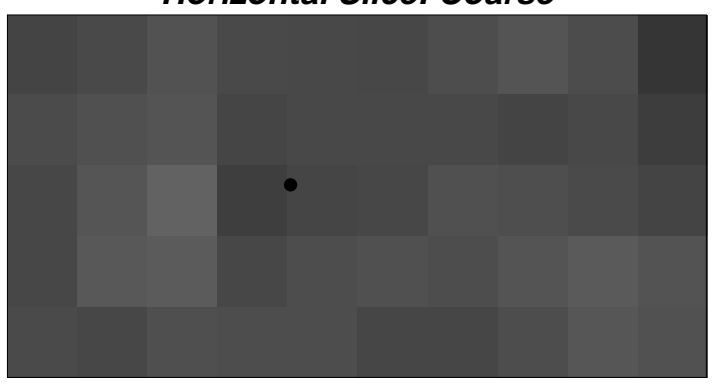

Vertical Slice: Coarse

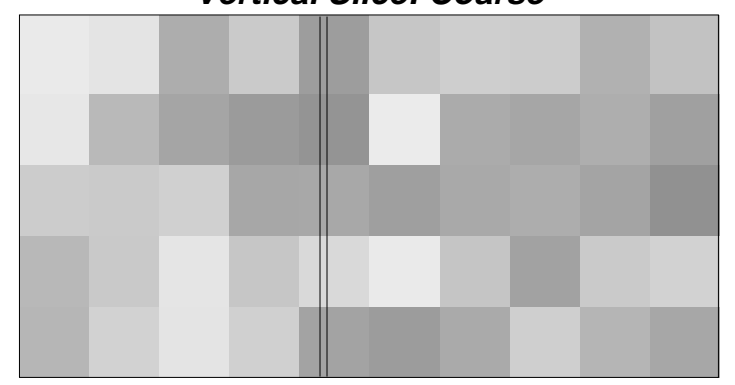

Horizontal Slice: Medium (power average)
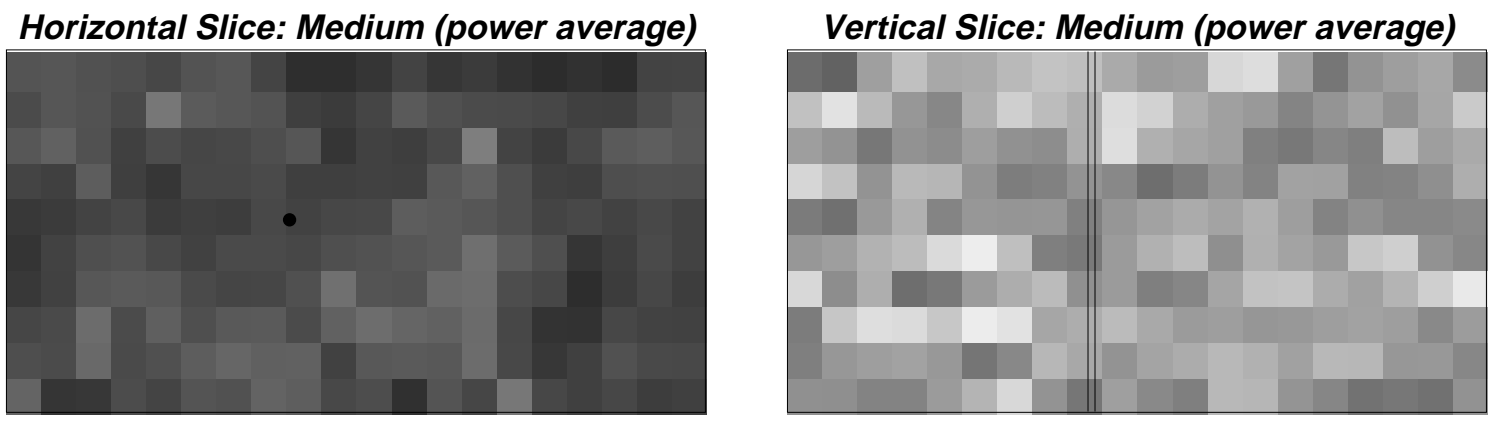

Fig. 7.4: Horizontal and vertical permeability maps of realization 12 for fine, medium-f, coarse-f, and medium-p grids. Slices are for the plane of the well. 

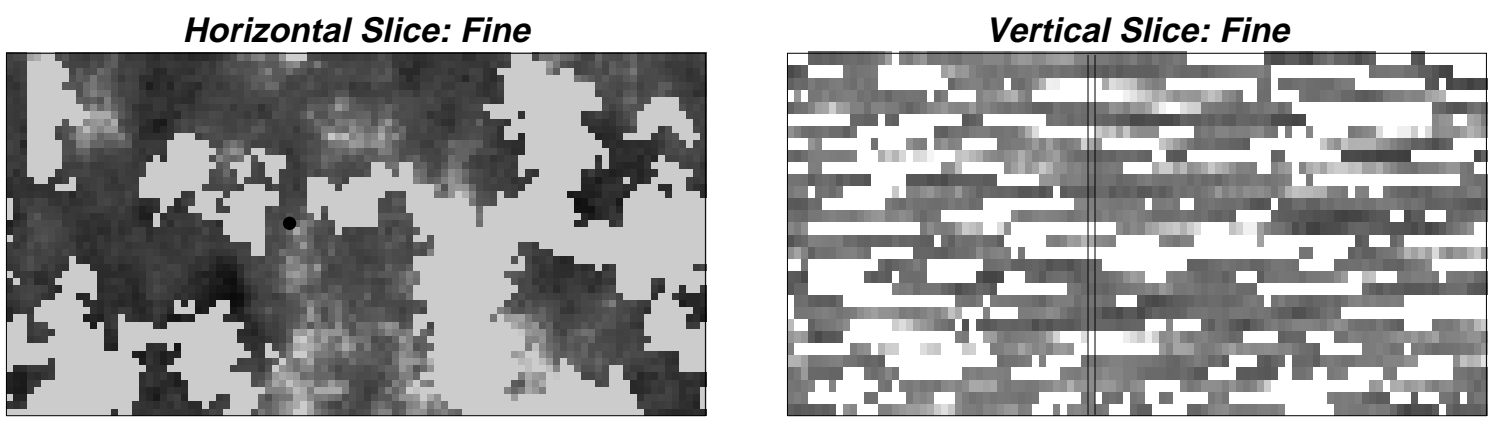

Horizontal Slice: Medium

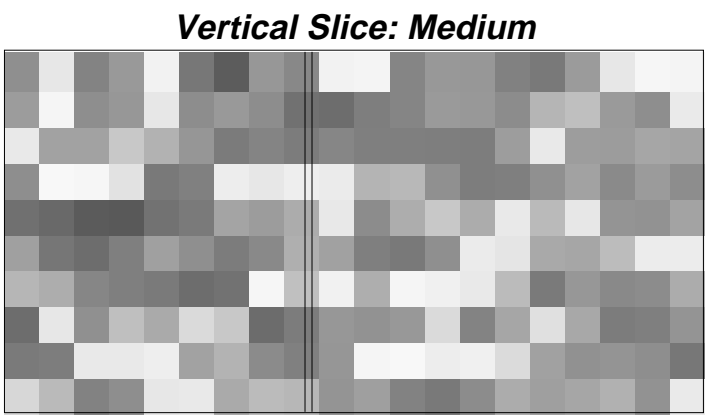

Horizontal Slice: Coarse

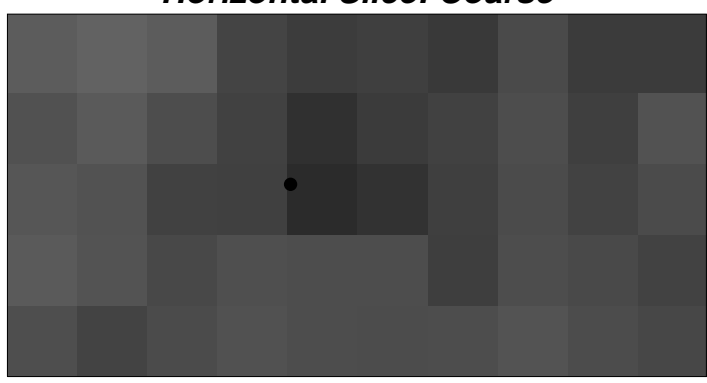

Vertical Slice: Coarse

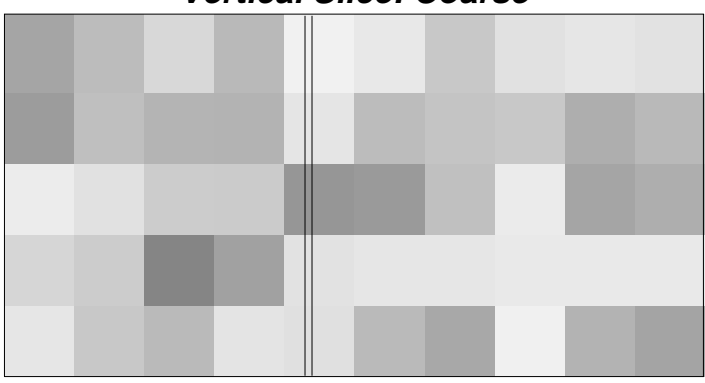

Horizontal Slice: Medium (power average)

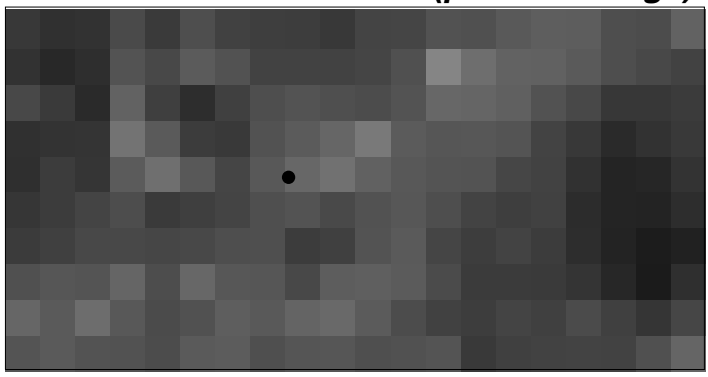

Vertical Slice: Medium (power average)

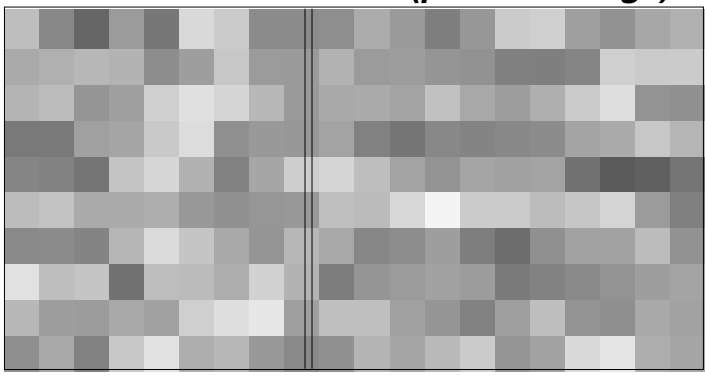

Fig. 7.5: Horizontal and vertical permeability maps of realization 19 (base case) for fine, medium-f, coarse-f, and medium-p grids. Slices are for the plane of the well 


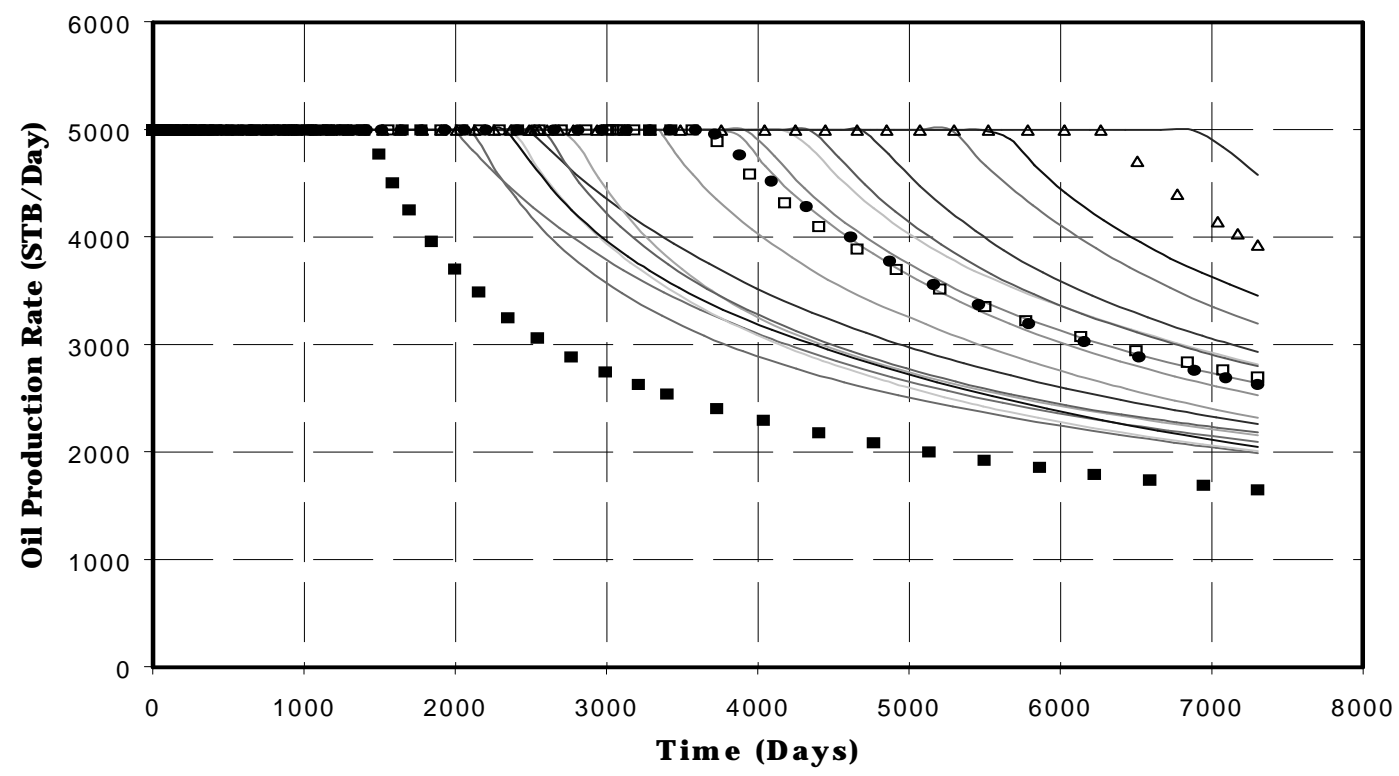

Fig. 7.6: Oil production rate for 20 realizations on Medium-f grid. Symbols identify the four images from Figures 7.2-7.5. - $\bullet$ is realization $3, \Delta$ is realization $9, \square$ is realization 12 , and $\square$ is realization 19 which is the base case

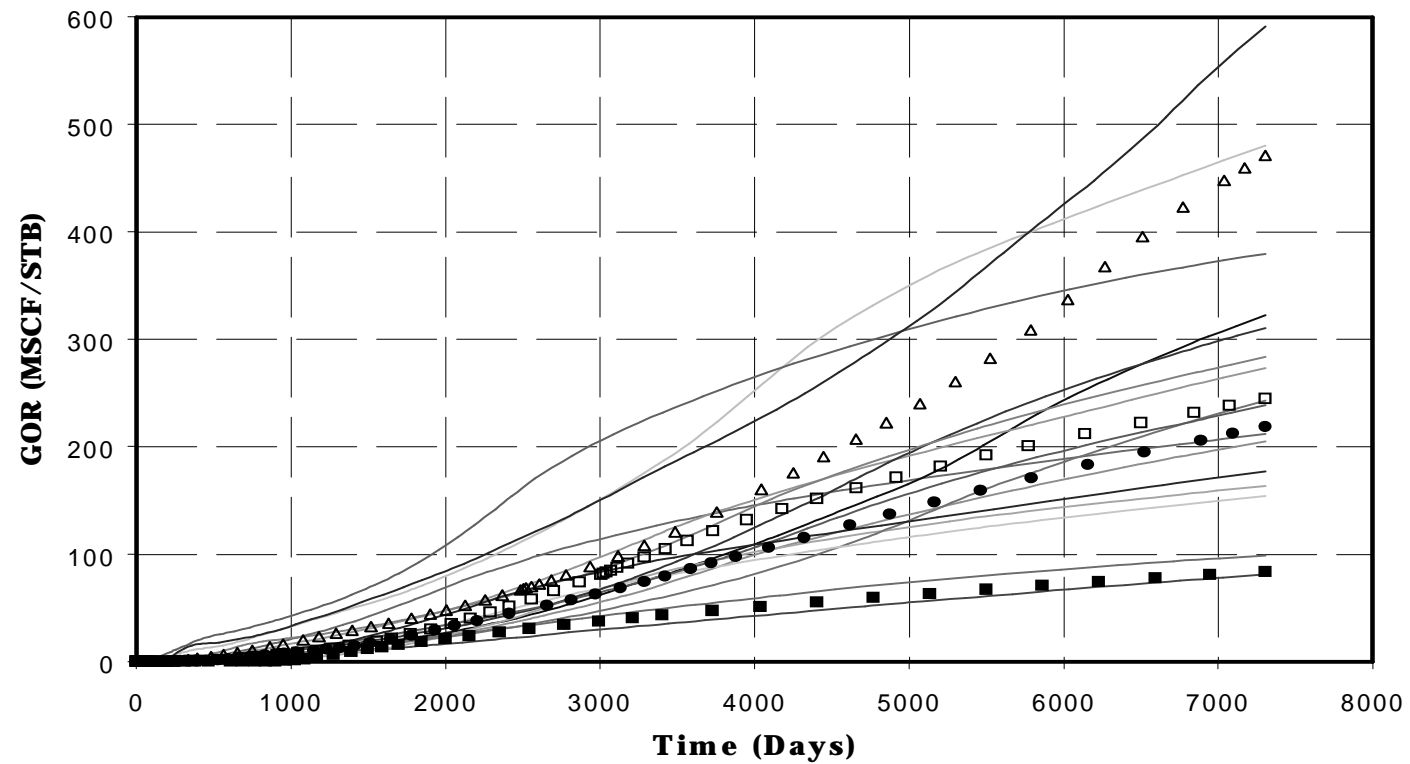

Fig. 7.7: GOR for 20 realizations on Medium-f grid. Symbols identify the four images from Figures 7.2-7.5. - • is realization 3, $\Delta$ is realization $9, \square$ is realization 12 , and $\square$ is realization 19 which is the base case 


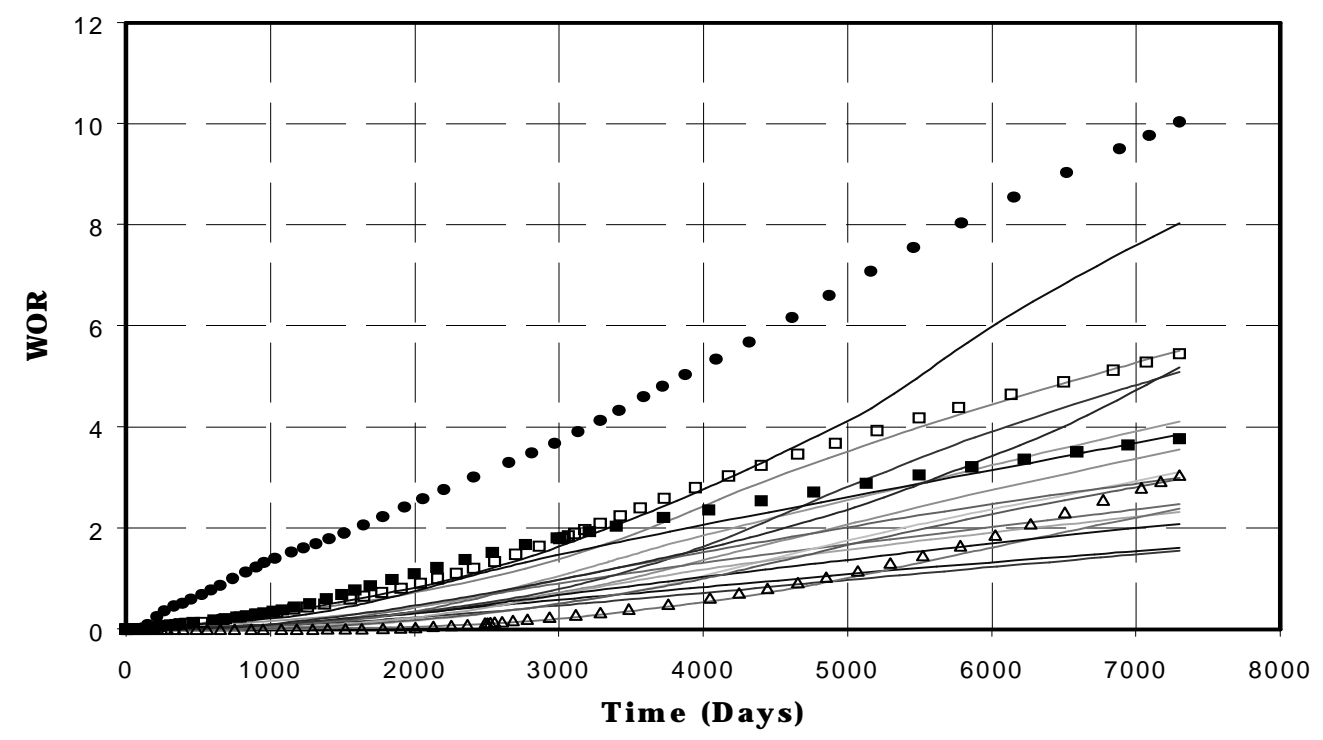

Fig. 7.8: WOR for 20 realizations on Medium-f grid. Symbols identify the four images from Figures 7.2-7.5. $-\bullet$ is realization $3, \Delta$ is realization $9, \square$ is realization 12 , and $\square$ is realization 19 which is the base case

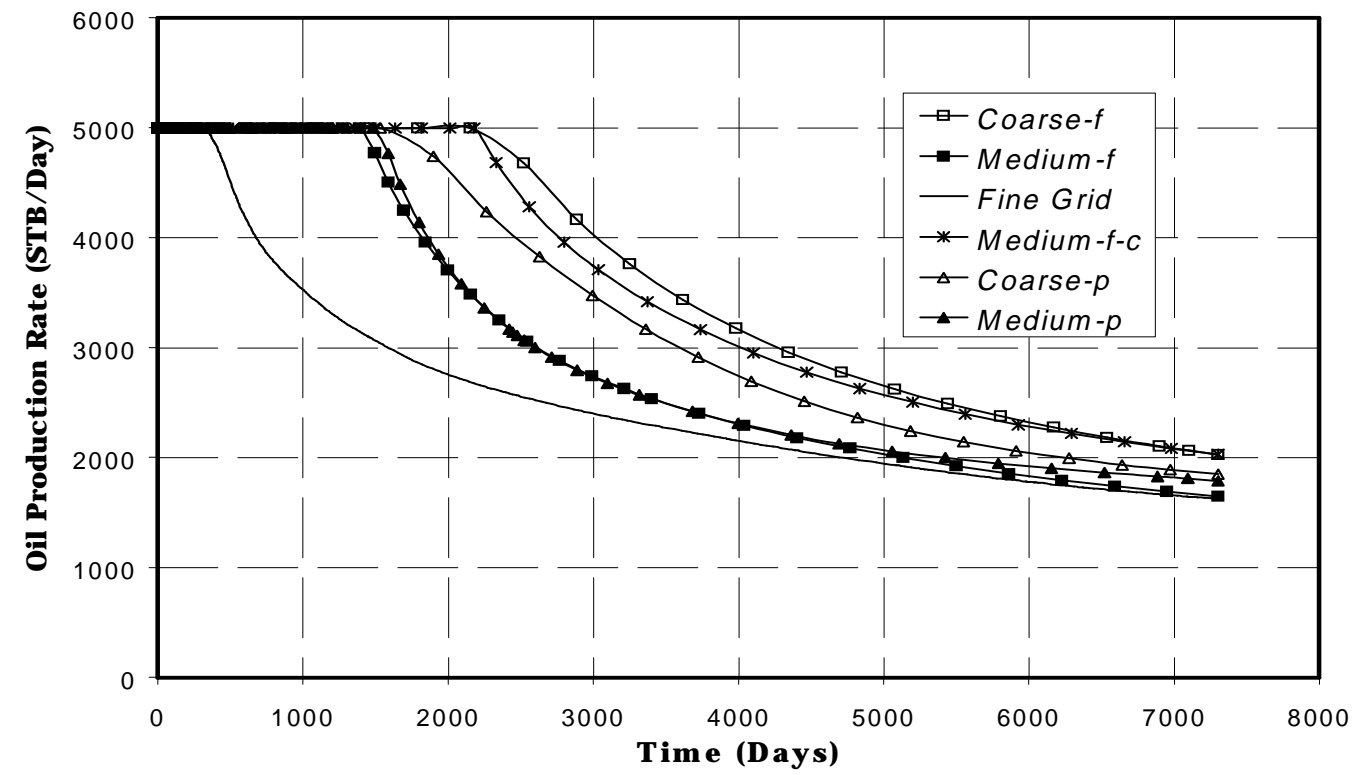

Fig. 7.9: Comparison of oil production rates from different grids for the base case (realization 19) 


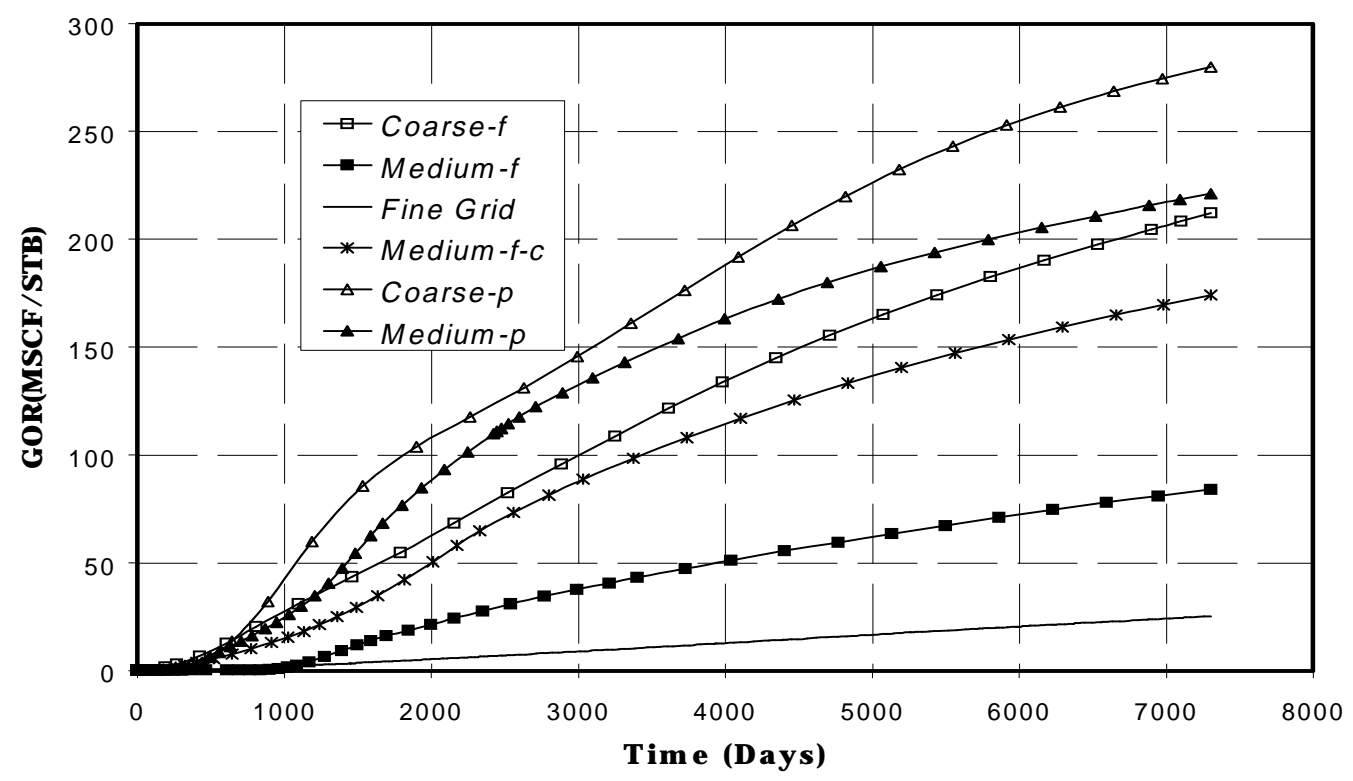

Fig. 7.10: Comparison of gas/oil ratio (GOR) from different grids for the base case (realization 19)

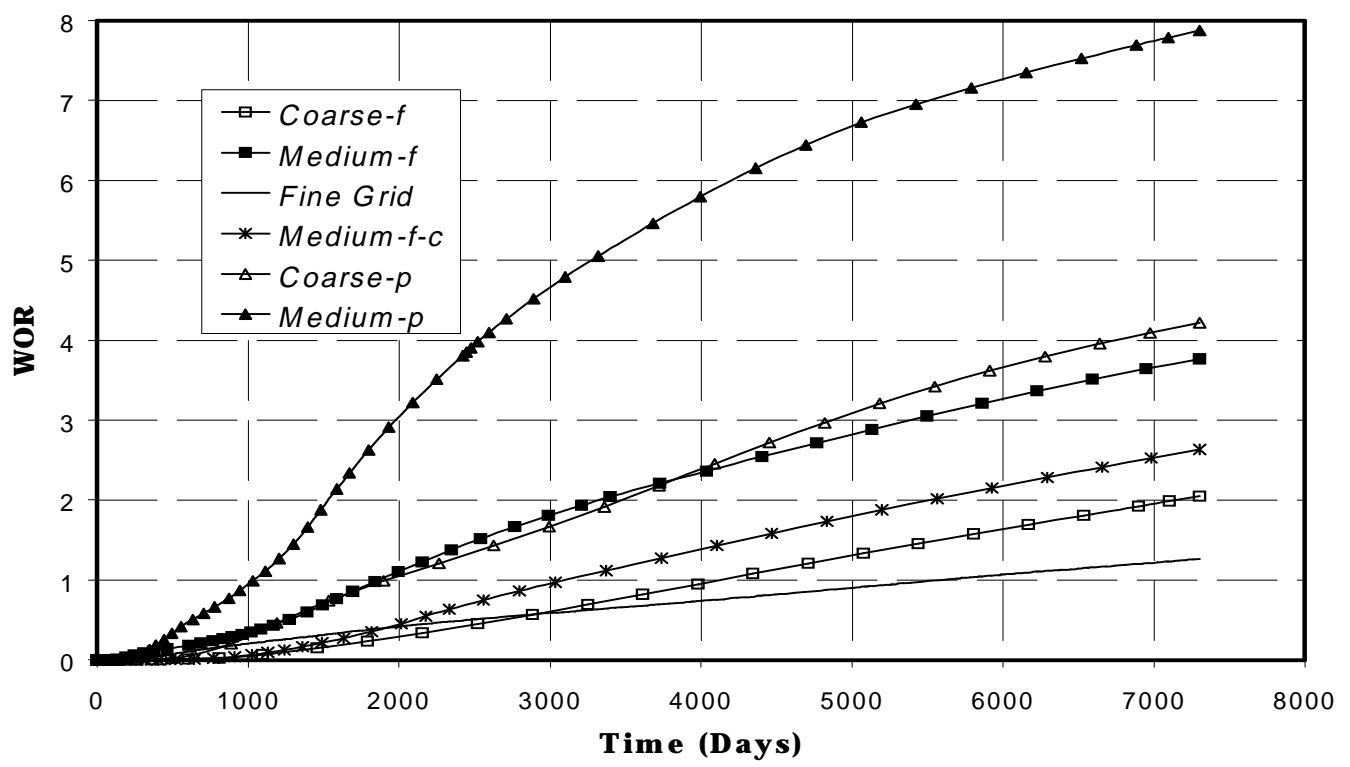

Fig. 7.11: Comparison of water/oil ratio (WOR) from different grids for the base case (realization 19) 
Table 7.2: Summary of Simulation Results

\begin{tabular}{|c|c|c|c|c|c|c|}
\hline & $\begin{array}{l}\text { Cumulative } \\
\text { Oil Production } \\
\text { (plateau period) } \\
\text { MMSTB }\end{array}$ & $\begin{array}{l}\text { Time to Reach } \\
\text { Oil Rate of } \\
\text { 3,000 STB/Day } \\
\text { Days }\end{array}$ & $\begin{array}{l}\text { GOR at } \\
6000 \text { Days } \\
\text { MSCF/STB }\end{array}$ & $\begin{array}{l}\text { WOR at } \\
6000 \text { Days }\end{array}$ & $\begin{array}{l}\text { WBHP at } \\
2000 \text { Days } \\
\text { Psia }\end{array}$ & \\
\hline Maximum & 30.94 & 9726 & 384 & 7.44 & 2109 & \multirow{3}{*}{$\begin{array}{l}\text { Coarse Grid } \\
\text { flow based }\end{array}$} \\
\hline Minimum & 7.40 & 3753 & 74 & 0.89 & 1500 & \\
\hline Mean & 18.09 & 6363 & 168 & 1.96 & 1823 & \\
\hline Maximum & 34.35 & 9706 & 426 & 8.23 & 2159 & \multirow{4}{*}{$\begin{array}{l}\text { Medium Grid } \\
\text { flow based }\end{array}$} \\
\hline Minimum & 6.95 & 2618 & 67 & 1.23 & 1500 & \\
\hline Mean & 18.20 & 5948 & 213 & 3.10 & 1856 & \\
\hline Spread & 27.40 & 7088 & 359 & 7.10 & 659 & \\
\hline Maximum & 23.61 & 8275 & 806 & 11.13 & 2118 & \multirow{4}{*}{$\begin{array}{l}\text { Medium Grid } \\
\text { power avg }\end{array}$} \\
\hline Minimum & 7.41 & 2601 & 172 & 3.59 & 1500 & \\
\hline Mean & 12.76 & 4467 & 426 & 6.90 & 1730 & \\
\hline Spread & 16.20 & 5674 & 634 & 7.54 & 818 & \\
\hline
\end{tabular}


These results show that the oil rate is more sensitive to upscaling than to gridblock size. However, GOR and WOR are highly sensitive to both the block size and upscaling. This is confirmed by other work (not reported here) where we have found that cresting calculations require very fine grids. It is also clear that the geological description has a huge influence on results. In particular, we see that the spread in results decreases as reservoir parameters are upscaled to coarser and coarser grids, and power law averaging reduces the spread as compared to flow based upscaling. The spread in predictions based on just 20 stochastic images is huge and it is likely to increase as more images are processed. The other interesting observation is that a smaller spread does not necessarily mean lower uncertainty. In other words, the distribution of uncertainty generated by repeated flow simulations may not span the true or full uncertainty because of the assumptions made in the stochastic model and/or the method used for upscaling. In the example discussed here, the flow based upscaling (considered to be the more reliable method) gives a bigger spread in results than simpler power law averaging. Neither of the two upscaling methods is exact.

\subsection{Influence of Model Assumptions}

\subsubsection{Well Models}

All simulators use simplified models to relate the wellblock pressure to the pressure of the well in that block. Here we will show the effect of using an inappropriate well model. The sources of uncertainty in the well model are: saturation gradients that cause the effective phase permeabilities for the well region to be different from the corresponding values for the well grid block, effective absolute permeabilities for the well region may be different from the average values for the block, the effective block radius $\left(r_{0}\right)$ may be in error because default procedures in simulators are based on assumptions that are more suitable for typical vertical wells than for horizontal wells, and the effective skin may not be known. All of these factors are not likely to cause the well index to increase or decrease by a factor of more than 5 times the default value. We have done simulations by changing the well index by a factor of 5, 0.2, 1.2 and 0.8 . As expected the oil production rate, GOR, WOR are all highly sensitive to the WI. The effect of changes in WI on production rate and GOR for the base case medium-f grid are shown in Figure 7.12 . 


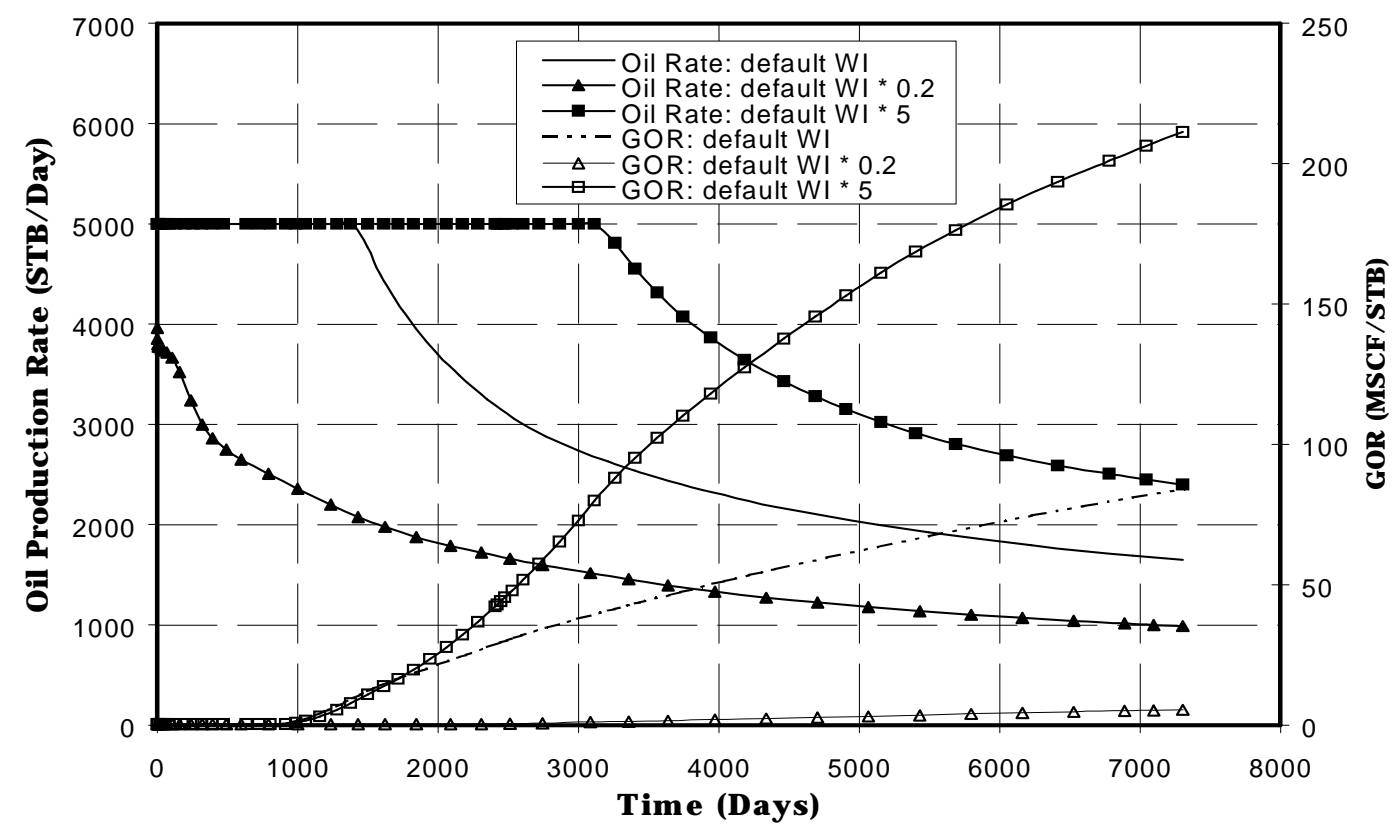

Fig. 7.12: Effect of well index (WI) on oil production rate and GOR for the base case (realization 19)

\subsubsection{Effect of Wellbore Pressure Drop on Well Performance}

Another factor in well modeling that is often ignored is the pressure drop in the well. This is only important in cases where the reservoir permeability is high and the drawdown is small. We have used a high pipe roughness of $1 \mathrm{~mm}$ in the medium- $f$ grid base case to see the effect of well pressure drop on results. The pressure drop calculation method is the homogeneous (no-slip) model in the Eclipse $(1995)^{8}$ simulator. These two values of roughness cause a pressure drop in the well at 6,000 days that is approximately $18 \%$ of drawdown. The maximum reduction in oil rate is about $12 \%$ when friction is included over when friction is ignored. Clearly not as major a factor in this case as reservoir heterogeneties. The greatest effect of wellbore pressure drop is on GOR. While in the homogeneous case the wellbore pressure drop causes the gas to breakthrough earlier than when this pressure drop is ignored, the behavior for the base case is opposite.

\subsubsection{Analytical Cresting Models}

Often analytical models are used to assess the tendency of water and gas to crest into a horizontal well. This is done because the simulation of cresting requires very fine grids. The analytical cresting models are based on assumptions that are generally different from those in simulation studies or real fields. Also, analytical models can only produce critical rates or time for 
breakthrough, not the behavior for super-critical rates. Arbabi and Fayers (1995) ${ }^{7}$ have shown that different semi-analytical models presented in the literature can produce results for critical rates that are different by a factor of 24 . They have proposed a new model that produces essentially the same results as careful simulations that mimic the assumptions in the analytical model. Here we present an example where we have used the technique developed by Arbabi and Fayers $(1995)^{7}$ to calculate the critical rate for our problem, under the assumption of steady-state, using average permeabilities of $433 \mathrm{mD}$ in the horizontal direction and $14 \mathrm{mD}$ in the vertical direction. The optimum well location, defined as the location that gives the same critical rate for both the gas and water interfaces, is predicted to be about $30 \mathrm{ft}$ from the gas/oil contact with a critical oil rate of about 68 barrels per day. Theory shows that for the no-flow boundaries used in simulation, there is no critical rate. Simulations with a rate of 50 barrels per day (critical rate of the well in the middle of the reservoir) show that indeed this is true, but the gas and water breakthroughs occur after 3,000 years for the "optimum" well location, and when the well is located in the middle of the reservoir water breakthrough occurs at about 1,800 years and gas at about 4,300 years. As expected, when the simulations are done with medium- $f$ grid breakthroughs occur earlier. We have also done simulations with the production rate of 5,000 barrels per day and the two well locations. Again, as expected, moving the well to the $30 \mathrm{ft}$ location delays water breakthrough and reduces gas breakthrough times. The overall conclusion from this part of our work is that critical cresting rate solutions available in the literature have limited practical utility, because real situations normally have different boundary conditions and furthermore economical production rates are usually much higher than critical rates.

\subsubsection{Productivity Models}

The most common approach used to compare the performance of horizontal and vertical wells is the use of single phase analytical solutions for steady-state or pseudo steady-state flow in homogeneous media. The most popular of these is Joshi's equation. ${ }^{2,3}$ Since our problem does not reach steady-state it is not appropriate to directly use Joshi's equation. A more general transient analytical model based on the work of Babu and $\mathrm{Odeh}^{9}$ is used here with infinite conductivity in the well. In Figure 7.13 we compare analytical and simulation results. As expected the agreement between the numerical and analytical models for the single phase case is excellent. However, for the three-phase case (with the gas cap and the aquifer included) the simulation results (homogeneous properties, medium grid) are very different from the single phase results. The PI's calculated for various situations are given in Table 7.3. The PI depends on the model (analytical or numerical, boundary conditions, single or multiphase flow) used and the conditions in the reservoir (transient, pseudo steady-state, steady-state). These results clearly show the inappropriateness of using a steady-state analytical model for predicting horizontal well performance. Furthermore, we observe that the results are very different when pressure support is provided by the gas and water zones. 


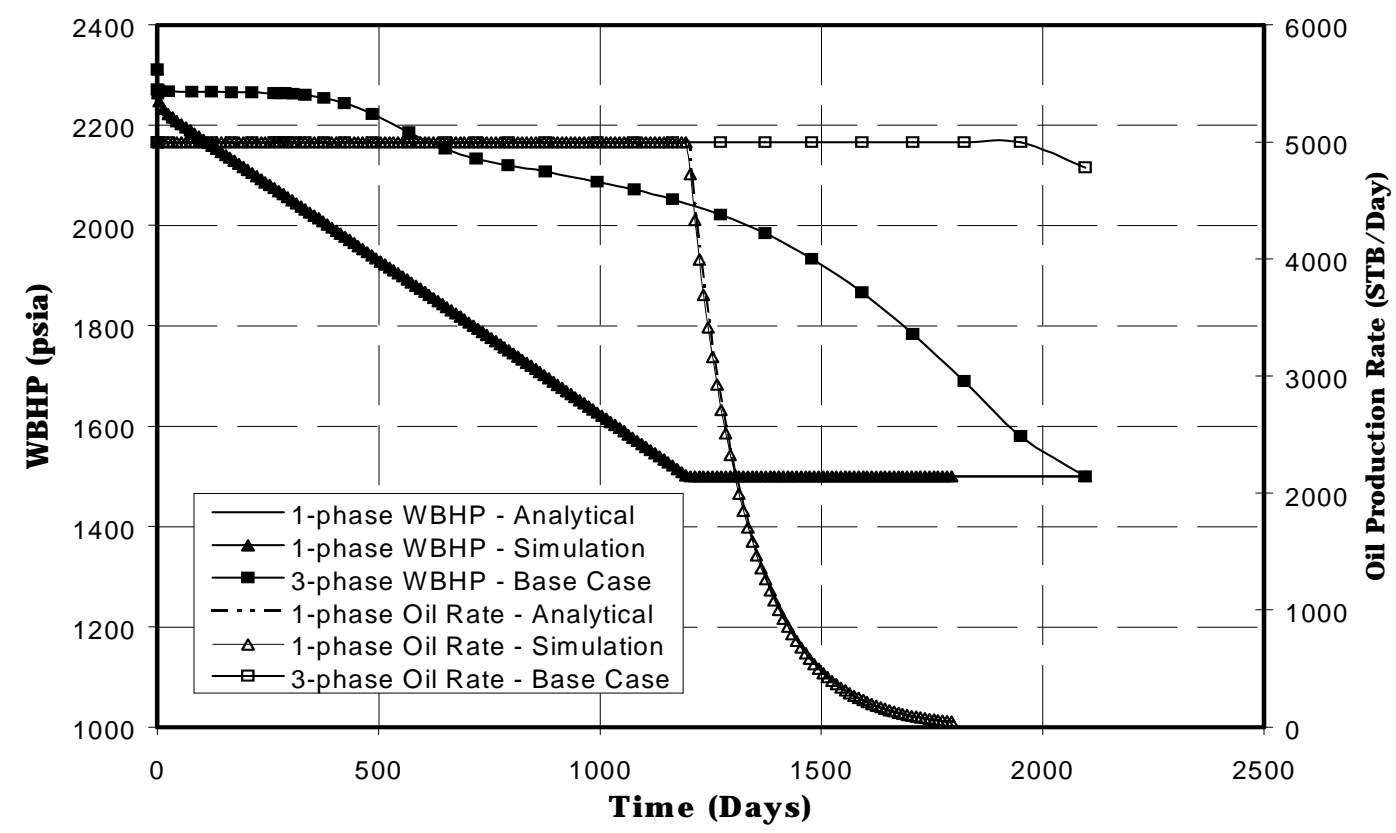

Fig. 7.13: Comparison between single phase (analytical and simulation) and three-phase medium grid simulation with homogeneous properties

Table 7.3 - Pl's Calculated from Various Methods

\begin{tabular}{|cccc|}
\hline Method \& Problem & \multicolumn{3}{c|}{$\begin{array}{c}\text { PI } \\
\text { (STB/Day/psi) }\end{array}$} \\
\hline & 100 Days & 1,400 Days & 4,000 Days \\
\hline $\begin{array}{c}\text { Babu and Odeh, 1-phase } \\
\text { (Transient) }\end{array}$ & $68.7^{*}$ & 67.5 & + \\
\hline $\begin{array}{c}\text { Simulation, 1-phase } \\
\text { (Transient) }\end{array}$ & $65.3^{*}$ & 63.9 & + \\
\hline $\begin{array}{c}\text { Joshi, 1-phase } \\
\text { (Steady State) }\end{array}$ & $18.9^{*}$ & 18.9 & + \\
\hline $\begin{array}{c}\text { Simulation, 3-phase } \\
\text { (Homogeneous Case) }\end{array}$ & $139.7^{*}$ & $15.2^{*}$ & 3.7 \\
\hline
\end{tabular}

+ no oil production at the fixed well bottom hole pressure of $1500 \mathrm{psi}$

* oil production at constant rate of 5000 STB/Day 


\subsubsection{Effect of Well Type}

In this section, we present and discuss results obtained when the horizontal well is replaced by a vertical well. The vertical producer is fully completed in the oil zone and two situations are considered: (1) the vertical well passes through the heel of the horizontal well discussed previously, and (2) the vertical well is moved to another location near the upper right hand corner of the reservoir. In each case, simulations with the previous twenty realizations were repeated on medium- $f$ grids. Figures 7.14-7.16 display the oil production rate, GOR, and WOR for the first case. In this case, all fine stochastic images have the same permeability and porosity at the well. The upscaled images do not have this property in common. Nonetheless, a much narrower spread is seen among various realizations in comparison with the results from the horizontal well (Figs. 7.6-7.8). This narrower spread is somewhat expected but perhaps what is surprising are lower GOR and WOR of the vertical well compared to the horizontal well. One reason for this behavior is provided by oil production rates in Figs. 7.8 and 7.14. From Fig. 7.14, it can be seen that all vertical wells have switched to bottomhole pressure control after 2178 days. On the other hand, horizontal wells can sustain the specified constant production rate of 5000 barrels per day up to 6870 days (Fig. 7.8). This means that vertical wells have lower production rates than horizontal wells. Obviously, this implies lower WOR and GOR for vertical wells. In fact, realizations with similar production rate history indicate lower or close WOR and GOR with horizontal well. Our base case happens to be such a realization. The base case with horizontal well goes under the bottomhole pressure control at 1495 days while it does the same at 773 days with the vertical well. The production rates at the end of 20 years are respectively $1650 \mathrm{STB} / \mathrm{day}$ and 1633 $\mathrm{STB} /$ day with horizontal and vertical wells. The corresponding GOR and WOR are 84 STB/MSCF and 3.77 for the horizontal well and 318 STB/MSCF and 3.63 for the vertical well.

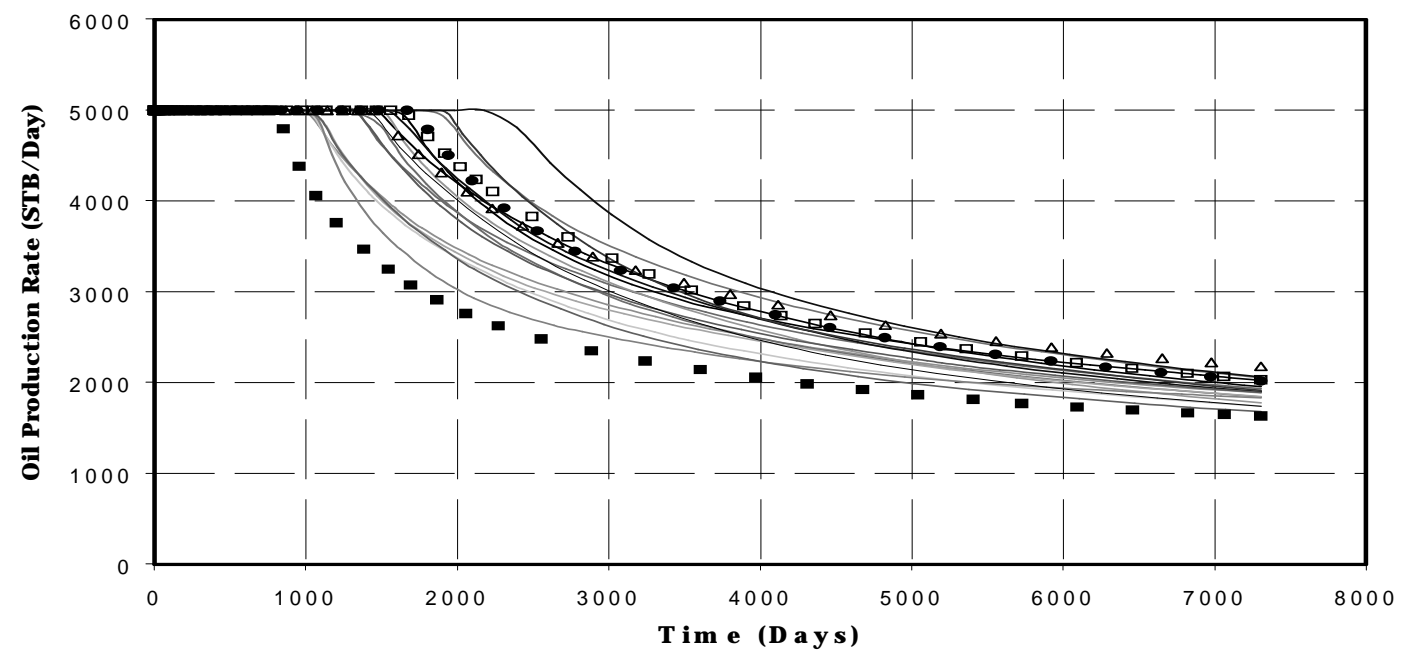

Fig. 7.14: Oil production rate for 20 realizations on Medium- $f$ grid for vertical well case (1). Symbols identify the four images from Figures 7.2-7.5. - $\bullet$ is realization $3, \Delta$ is realization $9, \square$ is realization 12 , and $\square$ is realization 19 which is the base case 


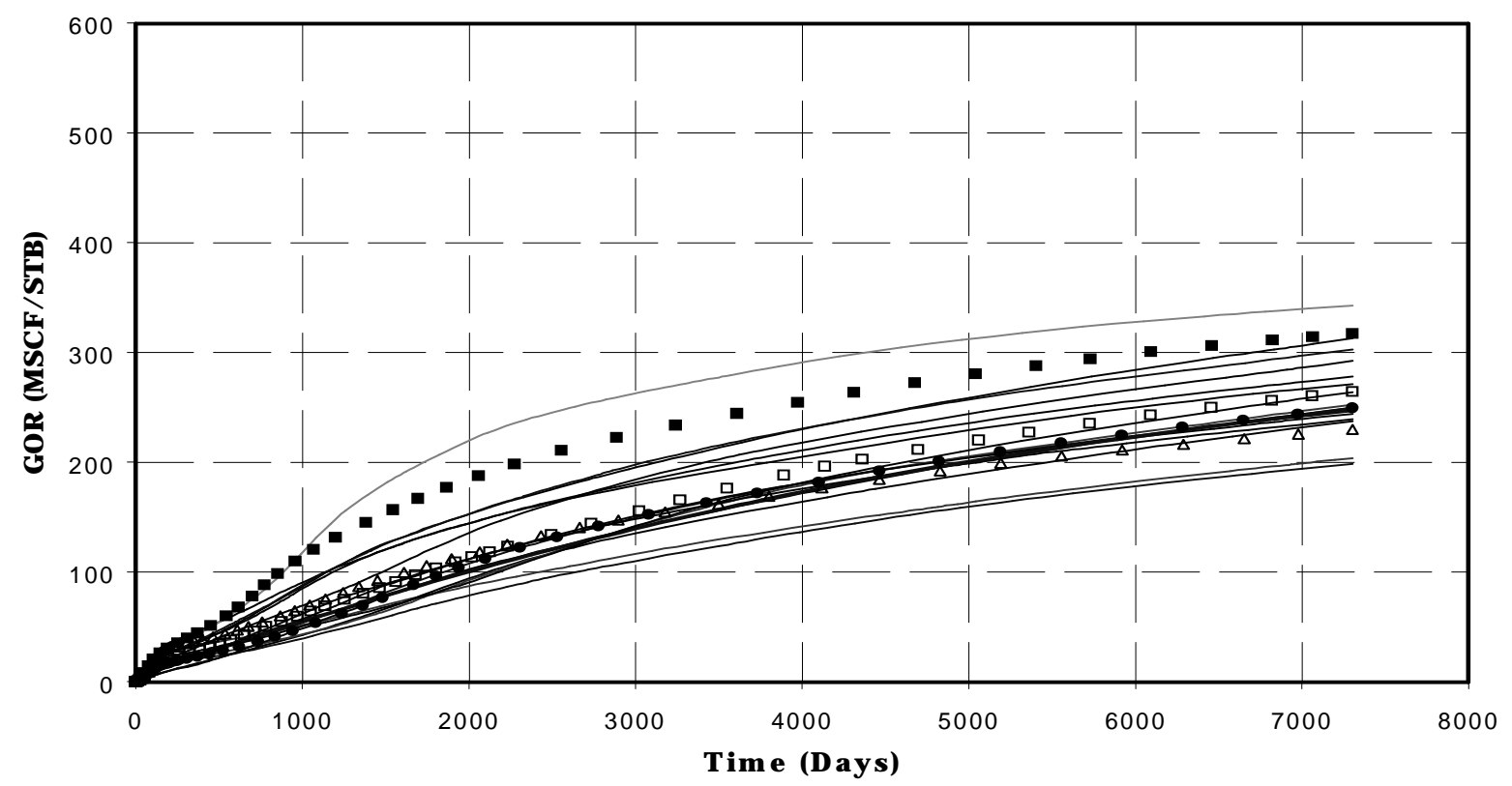

Fig. 7.15: GOR for 20 realizations on Medium-f grid for vertical well case (1). Symbols identify the four images from Figures 7.2-7.5. - $\bullet$ is realization $3, \Delta$ is realization $9, \square$ is realization 12 , and $\square$ is realization 19 which is the base case.

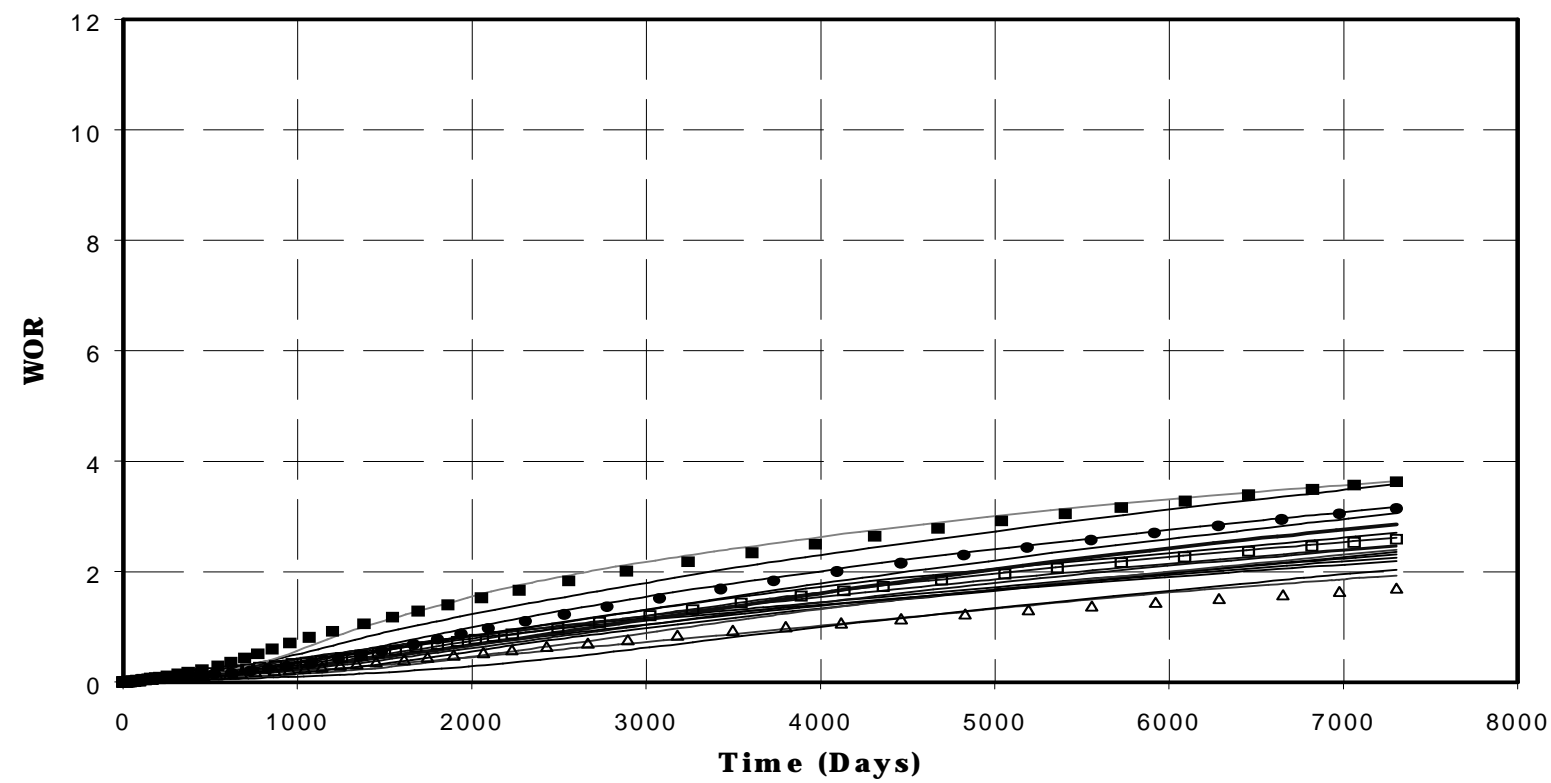

Fig. 7.16: WOR for 20 realizations on Medium-f grid for vertical well case (1). Symbols identify the four images from Figures 7.2-7.5. - $\bullet$ is realization $3, \Delta$ is realization $9, \square$ is realization 12 , and $\square$ is realization 19 which is the base case. 
In the second case, the vertical well is moved to location $\mathrm{x}=7500 \mathrm{ft}$ and $\mathrm{y}=1500 \mathrm{ft}$ near the upper right hand corner of the reservoir. Figures 7.17-7.19 show the results of the twenty realizations. Now every fine stochastic image is different at the well location. As a result the upscaled images show greater variability. This larger variation manifests itself in wider spread among different realizations as depicted in Figs. 7.17-7.19. One interesting observation is that the spread in results for this case is still less than that of the horizontal well. This points to the important role of geology along the length of a horizontal well, and emphasizes the need for proper modeling of this geology to reduce uncertainty.

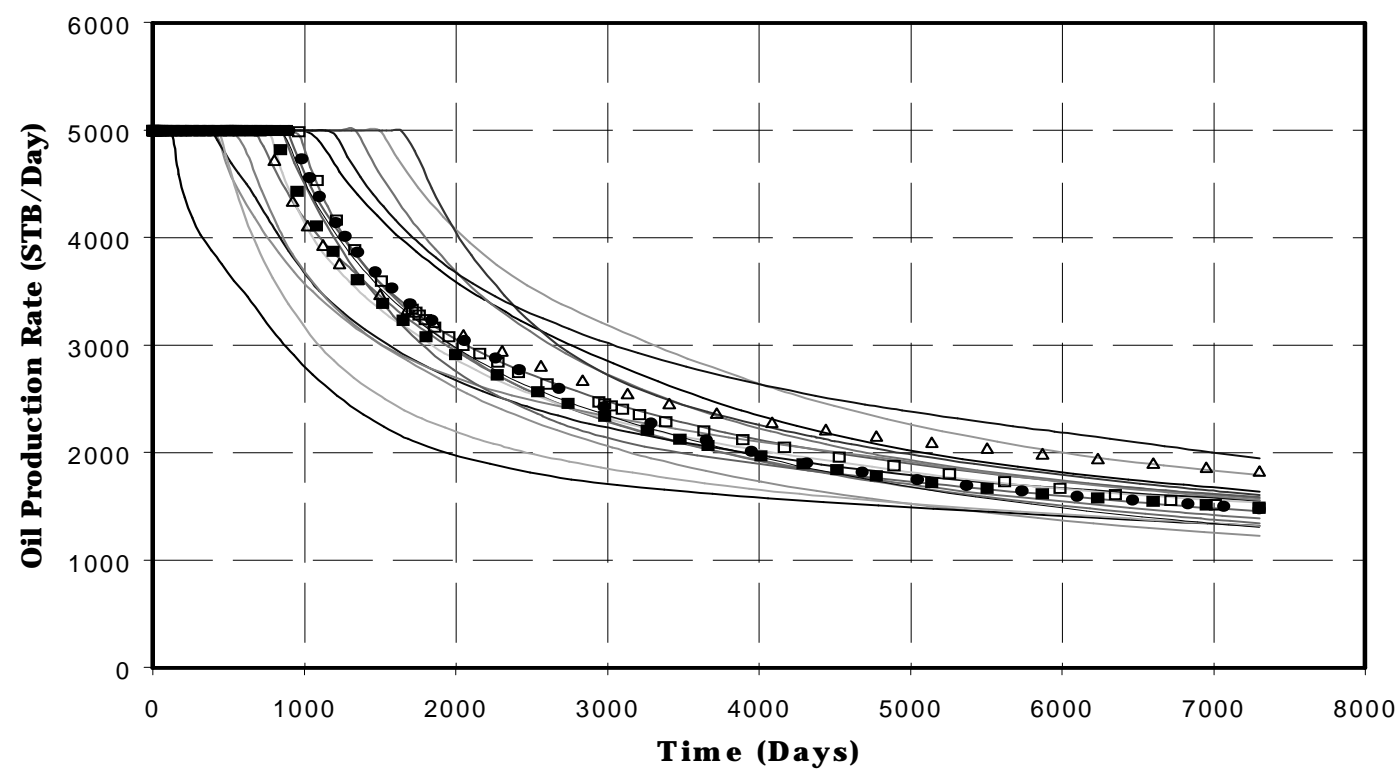

Fig. 7.17: Oil production rate for 20 realizations on Medium- $f$ grid for vertical well case (2). Symbols identify the four images from Figures 7.2-7.5. - $\bullet$ is realization $3, \Delta$ is realization $9, \square$ is realization 12 , and $\square$ is realization 19 which is the base case 


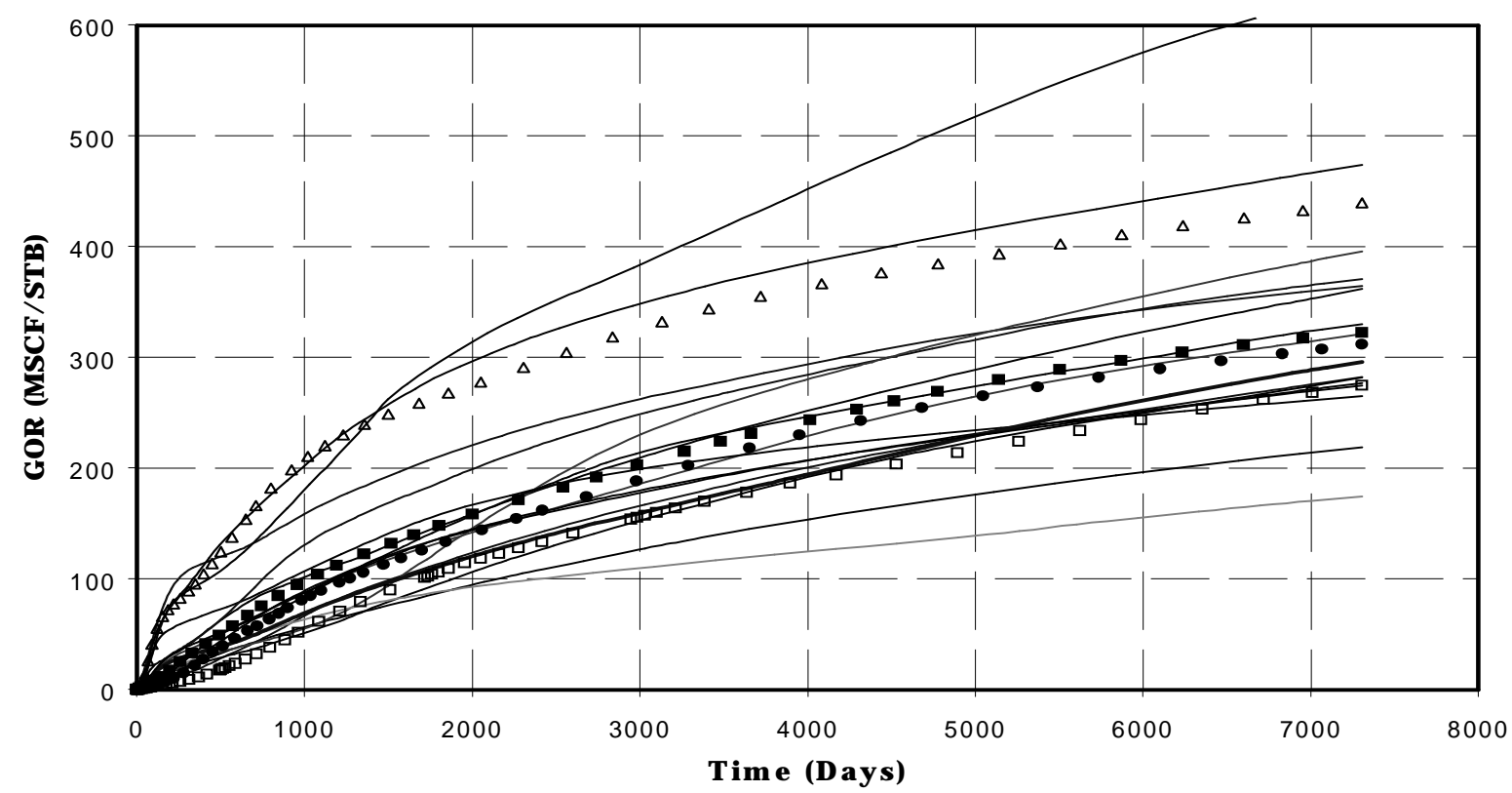

Fig. 7.18: GOR for 20 realizations on Medium-f grid for vertical well case (2). Symbols identify the four images from Figures 7.2-7.5. - $\bullet$ is realization $3, \Delta$ is realization $9, \square$ is realization 12 , and $\square$ is realization 19 which is the base case.

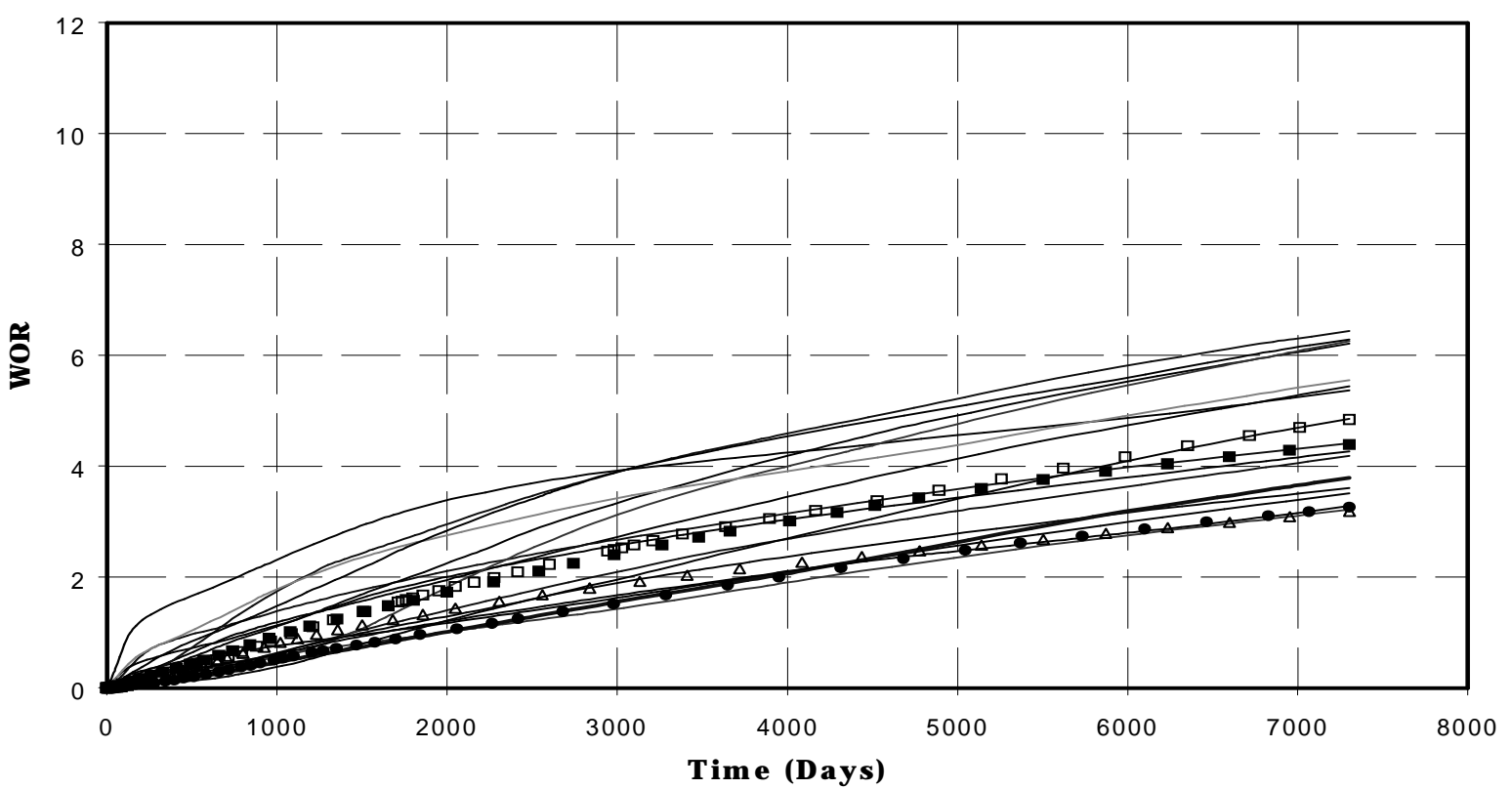

Fig. 7.19-GOR for 20 realizations on Medium-f grid for vertical well case (2). Symbols identify the four images from Figures 7.2-7.5. - $\bullet$ is realization $3, \Delta$ is realization $9, \square$ is realization 12 , and $\square$ is realization 19 which is the base 


\subsubsection{Other Factors}

Not all sources of errors or uncertainty have been investigated in this paper. Some of the other factors that may influence predictions but were not considered in our study are mentioned below:

1. Exact location of the well and the condition of the wellbore are often not known precisely.

2. Net-to-gross ratio, pore volumes and hydrocarbon volumes were all fixed.

3. Aquifer and gas cap sizes were fixed.

4. Influence of other wells in the field was not considered.

5. A very simple no-slip model for multiphase flow in the horizontal well was used.

6. Wellbore hydraulic calculations during multiphase flow in the well can be in error. In this study well pressure constrained at the well heel.

7. Only two upscaling techniques for absolute permeability were considered.

8. Relative permeabilities were not upscaled.

9. Grid alignment with the well can be a serious problem in multiple well studies.

10. The reservoir was assumed to not have any fractures.

11. A black-oil fluid description was used.

\subsubsection{Concluding Remarks}

The greatest source of uncertainty is reservoir description and how it is used in simulators. Integration of data through geostatistical techniques leads to multiple descriptions that all honor available data. The reality is never known. The only way to reduce this uncertainty is to use more data from geological studies, formation evaluation, high resolution seismic, well tests and production history to constrain stochastic images.

Even with a perfect knowledge about reservoir geology, current models cannot do routine simulations at a fine enough scale. Furthermore, we normally don't know what scale is fine enough. Upscaling introduces errors and masks some of the physical phenomenon that we are trying to model. The scale at which upscaling is robust is not known and it is probably smaller in most cases than the scale actually used for predicting performance. Uncertainties in the well index (WI) can cause errors in predictions that are of the same magnitude as those caused by reservoir heterogeneities.

Simplified semi-analytical models for cresting behavior and productivity predictions can be very misleading. 


\section{References}

1. Beliveau, D.: "Heterogeneity, Geostatistics, Horizontal Wells, and Blackjack Poker," JPT (December 1995) 1068.

2. Mullane, T. J., Churcher, P.L.,Tottrup, P. and Edmonds, A.C.: "Actual vs. Predicted Horizontal Well Performance, Weyburn Unit, S.E. Saskatchewan," JCPT, March 1996, Volume 35, No. 3, 24-30.

3. Joshi, S.D.: "Augmentation of Well Productivity Using Slant and Horizontal Wells." JPT (June 1988) 729.

4. Aziz, K.: “Ten Golden Rules for the Simulation Engineer,” JPT (Nov. 1989)1157.

5. Aziz, K.: “Advances in Horizontal Well Simulation," JCPT Distinguished Author Series Paper, (Nov. 1995) 34, No. 9, 14.

6. Deutsch, C.V. and Journel, A.G.: "GSLIB: Geostatistical Software Library and User's Guide." Oxford University Press, New York, 1992, 340

7. Arbabi, S. and Fayers, J.: "Comparative Aspects of Coning Behavior in Horizontal and Vertical Wells," paper presented at the $8^{\text {th }}$ European Symposium on Improved Oil Recovery, Vienna, Austria, May 15-17, 1995.

8. Eclipse Reference Manual, 95A Release, Intera Information technologies (now GeoQuest).

9. Babu, D. K. and Odeh, A. S.: "Productivity of Horizontal Wells," SPE Reservoir Engineering, (Nov. 1989) 417-421. 


\title{
8. Analysis of Gravity Drainage in Horizontal Wells
}

\author{
by Mahadevan Venkataraman, Dengen Zhou, Sepehr Arbabi, and \\ Khalid Aziz
}

\begin{abstract}
One of the major forces affecting the recovery of oil from reservoirs through horizontal wells is gravity. In this report, the effect of gas injection on the gravity drainage process in horizontal wells is discussed. The sweep efficiency of the gas injection process for different configurations of horizontal wells will be studied using a CT scanner. The viscous and gravitational forces coming into play in the reservoir are discussed. The experimental setup for this project is given and some results of simulation runs based on this physical model are provided.
\end{abstract}

\subsection{Introduction}

Horizontal wells are used extensively in recovering oil from reservoirs as they expose a larger amount of the formation to the well than vertical wells. One of the important forces in the recovery of oil using horizontal wells is gravity. This is especially important in case of heavy oils where the reservoir pressure is not sufficiently high to move the viscous oil towards the wellbore. Gravitational forces are most effective when the horizontal well is placed close to the bottom of the reservoir and the distance for the flow of oil through the porous medium is minimized.

Gas injection has been used widely to improve the recovery of oil. It can be used for maintaining pressure in the reservoir which in turn contributes to an increase in productivity or for miscible displacement. Nitrogen and carbon dioxide are the two commonly used nonhydrocarbon gases.

The significance of the gravity effects as well as the viscous effects in the gravity drainage processes are studied and a sensitivity analysis on the different factors affecting the production using a horizontal well is presented.

\subsection{Literature Survey}

Gravity drainage in horizontal wells for the recovery of oil has been studied with regard to the optimal positioning of the well and its advantages over the use of vertical wells. Meszaros et al.[1] experimentally studied the drainage of oil into horizontal wells using inert gas injection with oil viscosities varying from $1000 \mathrm{cp}$ to $4000 \mathrm{cp}$ and found that oil recovery increased from $50 \%$ to about $70 \%$. The stability of the gas front was also studied for two horizontal wells placed one on top of the other. Gas was injected through the upper well and the stability of the front was studied using a physical model. It was also found that it is more difficult to maintain a stable gas front in a 3-D model than in a 2-D model. The production was mainly due to gravity drainage at low flow rates and the oil recovery was high. In all the experiments, the initial production rate was found to be high due to gravity drainage and as 
the front moved down, the effect of gravity reduced and hence the production of oil reduced considerably. At very low injection pressure, the production rate of oil was very slow but a steady gas front was maintained. Also as the injection pressure was increased, the recovery of oil decreased. Both carbon dioxide and nitrogen were used in the study and in both cases the dissolution of the gas in oil was neglected.

Dykstra and Dickinson[2] studied the effect of dip, reservoir thickness, oil mobility and well spacing on production and recovery from horizontal and vertical wells. In this study, mobility ratio was considered to be uniform for both the horizontal as well as the vertical well case even though this assumption is not valid. The head of the fluid flowing into the well was calculated as the height from the wellbore to the gas oil contact. This head and flow rate were found to decrease with time. In the case of horizontal formations (no dip), there was no effect on the ratio of horizontal and vertical well flow rates with time. It was found that for very thick formations, vertical wells performed better than horizontal wells. For inclined formations, horizontal wells were found to produce more than vertical wells. The thickness affected the flow rate ratios of horizontal to the vertical wells. Also, in case of vertical wells the well spacing was found to be important.

Williams[3] studied the effects of horizontal well placement and gravity on sweep efficiency for a gas injection process. In the cases considered here, the injection rate and the production rate were made equal. Two cases considered were staggered pattern horizontal wells and two parallel horizontal wells. A viscous to gravity ratio was defined and the pore volumes produced were evaluated as a function of this ratio. It was found that sweep efficiency of a staggered pattern was less than that of adjacent horizontal wells. Enhancement of the gravity drainage process through hydrocarbon additives were also studied by Nasr et al.[4]. The recovery of oil by the use of horizontal wells were found to be always greater than $50 \%$ and the addition of Naptha to the steam injection process increased the recovery to $82.5 \%$. Extensive literature also exist on steam assisted gravity drainage and inert gas injection[5-7].

In most of the above cases, saturation profiles at different instances of time were not identified. The experiments were done using oil with high viscosities. The saturation profiles inside the models were not studied in these cases. The saturation profiles would indicate the regions where the oil recovery is high and where the residual oil saturations are high. In this report, the experimental setup to be used for studying the saturation profiles during the gravity drainage process using a CT scanner is discussed along with some simulation results based on the physical model.

\subsection{Viscous and Gravitational Forces}

In a gravity drainage process, the gravitational force is used to overcome the resistance to flow. The gravitational forces acting on the fluid are characterized by the gravity number which is the ratio of gravitational forces to viscous forces. On the other hand, capillary number is the ratio of capillary to viscous forces. The gravity and capillary numbers (Zhou et. al.[8]) are given as;

$$
N_{g}=\frac{k A \delta \rho g}{\mu_{0} q_{t}}
$$




$$
N_{c}=\frac{k A \delta p_{c}}{q_{t} \mu_{0} L}
$$

where $\mathrm{k}$ is the permeability of the medium, $\mathrm{A}$ is the cross-sectional area open to flow, $\delta \rho$ is the difference in the densities of oil and gas, $\mu_{0}$ is the oil viscosity, $q_{t}$ is the total flow rate, and $\delta p_{c}$ is the capillary pressure between oil and gas at a given oil saturation.

In a gravity drainage process, the gravity number should be much greater than the capillary number. The flow rate in the model should be adjusted such that $N_{g}>>N_{c}$. The gravity and capillary numbers are also used to scale up from the model to the field.

\subsection{Experimental Setup and Procedure}

The main aim of this study is to obtain sweep efficiencies for different horizontal well configurations. A Berea sandstone core (4 inches diameter and 2 feet long) will be used for this study and the core will be coated with epoxy. Inlet and outlet ports will be provided on diametrically opposite ends of the core as shown in Figure 8.1. The ends of the core will be sealed with a flow distributor. Decane will be used for the initial case. The core will be saturated with decane and the permeability of the core will be measured.

The gravity drainage process by itself would take a very long time. This is because as the height of the fluid decreases, the pressure head drops and it takes a very long time to drain the oil out. Instead, gas will be injected at a very low flow rate to speed up the recovery. Care would be taken to make sure that flow is dominated by gravitational forces. The gas used in this case is Nitrogen.

A CT scanner will be used to scan the core for saturations and the saturation profiles will be obtained at different time intervals and at different depth of the cores. These patterns will be matched with the initial dry pattern of the core and the saturation of oil will be obtained.

\subsection{Simulation of the Experiment}

Experiments which are to be conducted were first simulated with the help of the Eclipse commercial simulator. A lab scale study was done in order to estimate the injection flow rate of Nitrogen. The size of the core was 4 inches in diameter and $60 \mathrm{cms}$ in length. This was modeled using cubic grids of $1 \mathrm{~cm} \mathrm{x} 1 \mathrm{~cm} \mathrm{x} 1 \mathrm{~cm}$ each and no flow boundary conditions were applied to the boundary blocks. Transmissibilities of some boundary blocks were set to zero in order to approximate the cylindrical shape of the core. A horizontal injector was completed at the top and a horizontal producer was used at the bottom. The producing well was kept at atmospheric pressure and the pressure of the injection well was kept just above the atmospheric pressure (0.01 psi difference). The experiments were simulated using different permeabilities and injection pressures and the results that are provided are for the $300 \mathrm{mD}$ case. The typical values of porosity for Berea sandstone varies between 0.1 to 0.3. For this simulation, the value of porosity is taken to be 0.1 . Sensitivity runs were also done for different horizontal well configuration such as adjacent and staggered patterns.

A typical saturation profile after 0.4 days of gas injection is given in Figure 8.2. The

production rate of oil for horizontal injector placed at the top and horizontal producer placed 

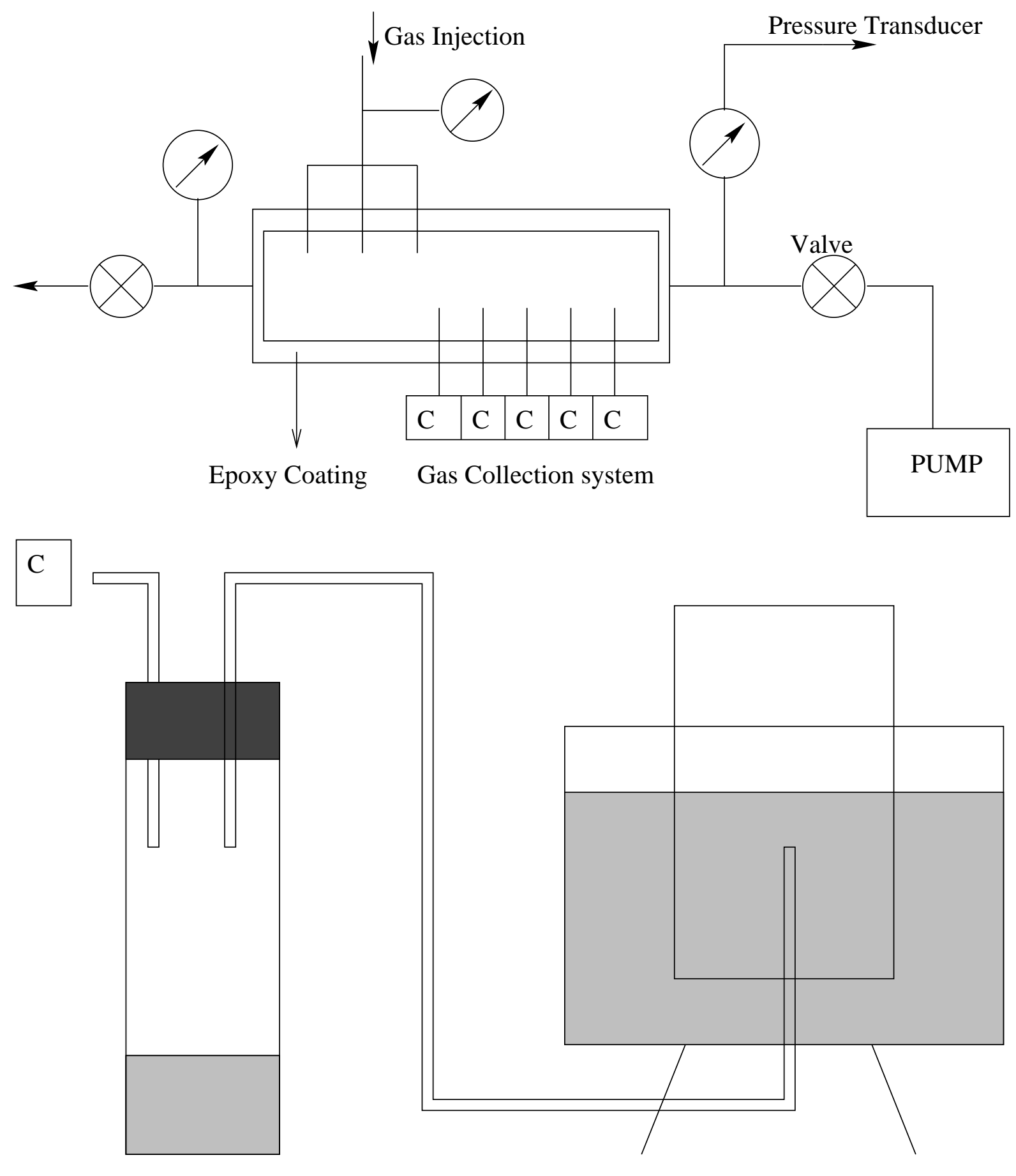

Liquid Separator

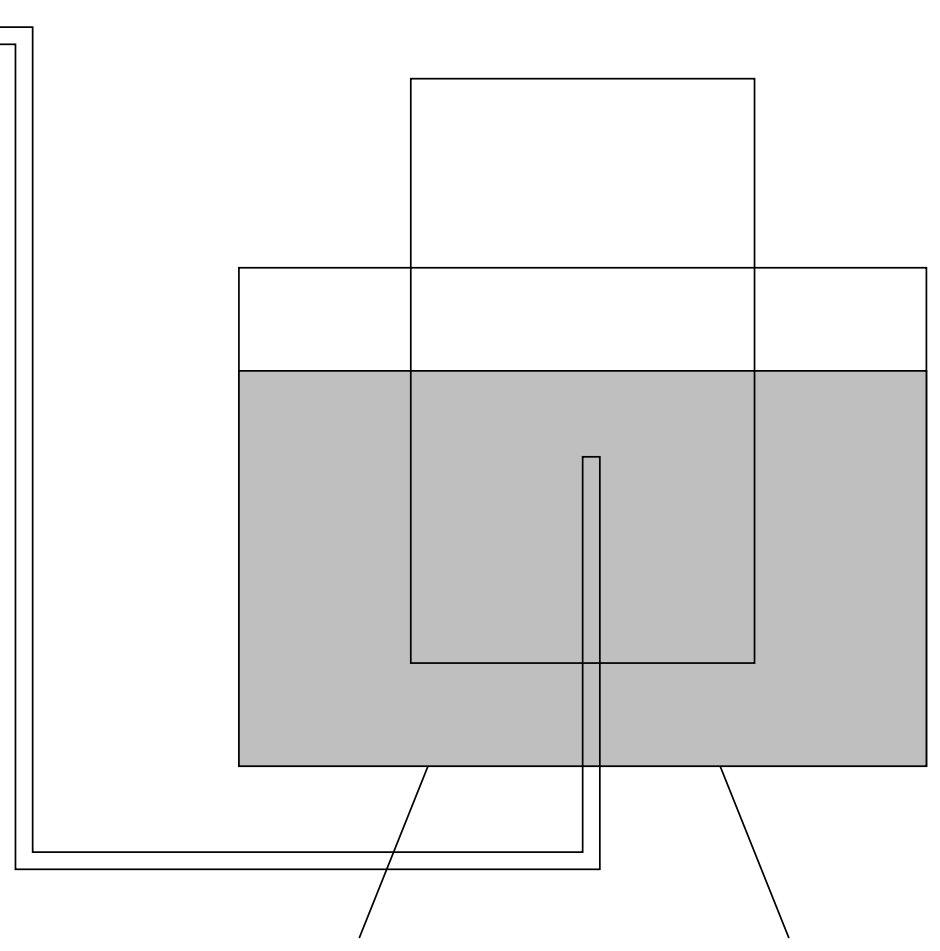

Gas flow rate measurement

Figure 8.1: Experimental Setup 
at the bottom is given in Figure 8.3. The shape of the production curve obtained was found to match with that of the general trend of production curves in gravity drainage processes. There is a sharp increase in the production rate at early times and the production falls off with time. In this case, horizontal wells were also modeled as a series of vertical wells. The results were found to be similar for both the cases.

Simulations were run using one vertical producer and one vertical injector. It can be seen that the sweep efficiency of the vertical wells is very low compared to the sweep efficiency of the horizontal well by comparing Figure 8.4 and 8.5. The sweep efficiency was found to be good at the top layers as the gas swept through all the top layers. But after gas break-through, the production of oil is reduced and residual oil saturation is high in some regions. The saturation profile indicating the sweep efficiency of the vertical wells can be seen in Figure 8.5.

The production rates for oil and gas are given in Figures 8.6 and 8.7. It can be seen that there is an increase in the production rate till gas breakthrough and after gas breakthrough there is a sharp decrease in the production rate of oil.

The saturation profiles obtained from the CT scanner will be matched to the saturation profiles from simulation. From the differences in the two saturation profile, it can be observed as to how the heterogeneities affect the sweep efficiency. Also, the effects of heterogeneities can be studied using a simulator. Sensitivity studies were also done using simulations and a similar trend in the production rates and saturation profiles were observed for different viscosities and permeabilities.

\subsection{Future Work}

Experiments are to be performed for the vertical wells case, staggered horizontal wells pattern and adjacent horizontal wells pattern. The saturation profiles from the experiment are to be compared with the profiles obtained from the simulation runs. The physical model results will be up-scaled to the field scale. Also the effect of heterogeneity on the gravity drainage process will be studied using the simulator. 


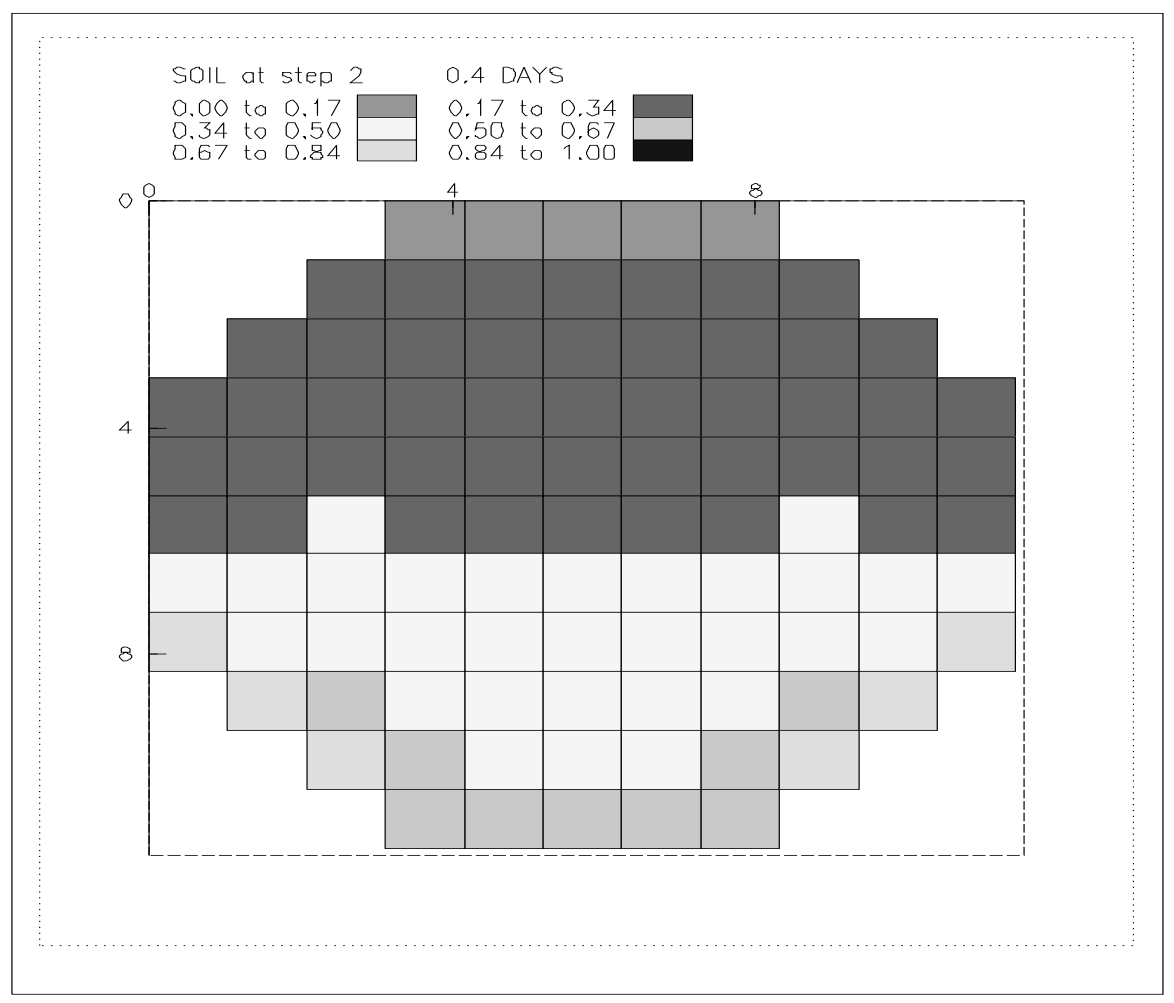

Figure 8.2: A Typical Saturation Profile after 0.4 Days

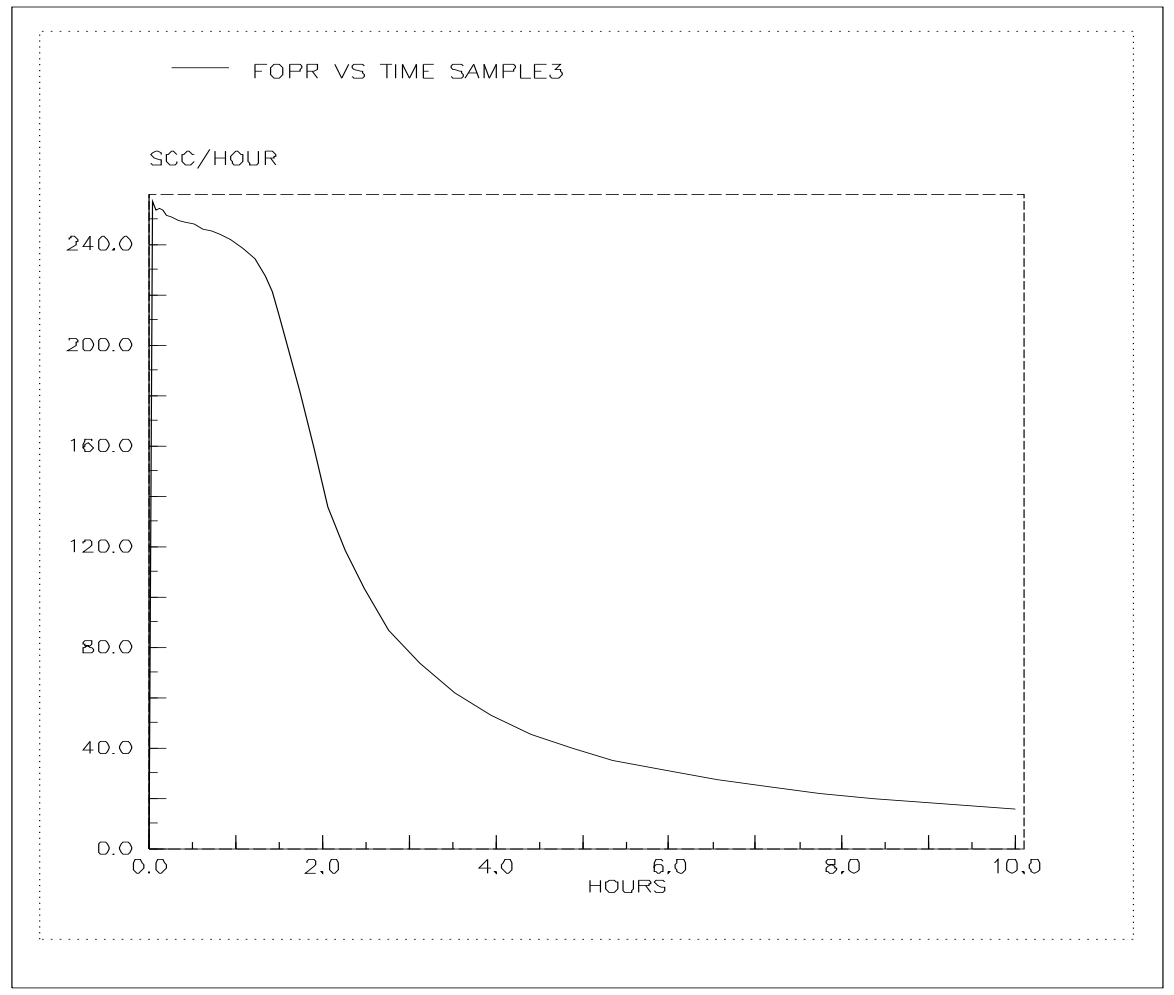

Figure 8.3: Production Rate of Oil versus Time for Parallel Horizontal Injector and Producer 


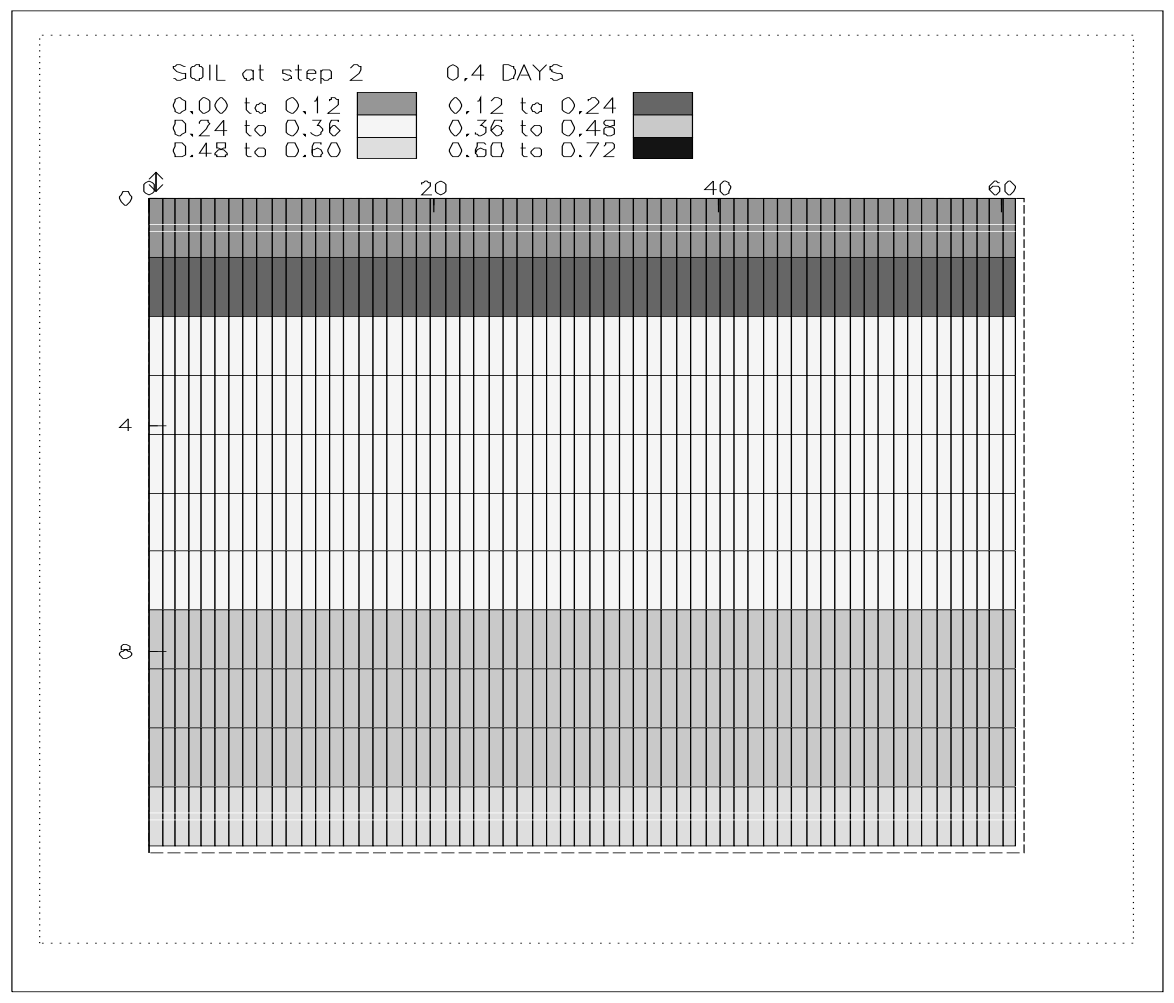

Figure 8.4: Saturation Profile in X-Z Direction for Horizontal Injector and Producer

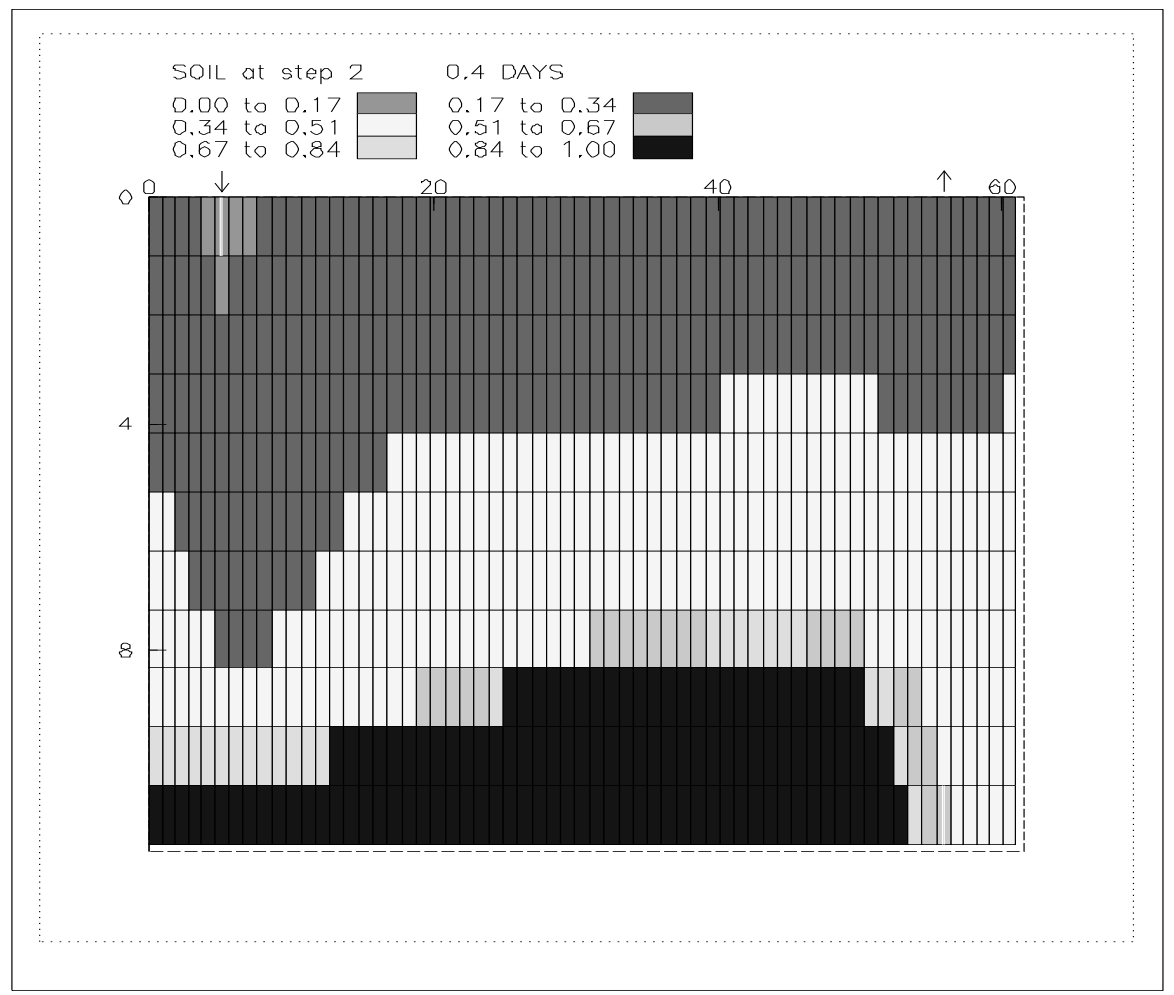

Figure 8.5: Saturation Profile in X-Z Direction for Vertical Injector and Producer 


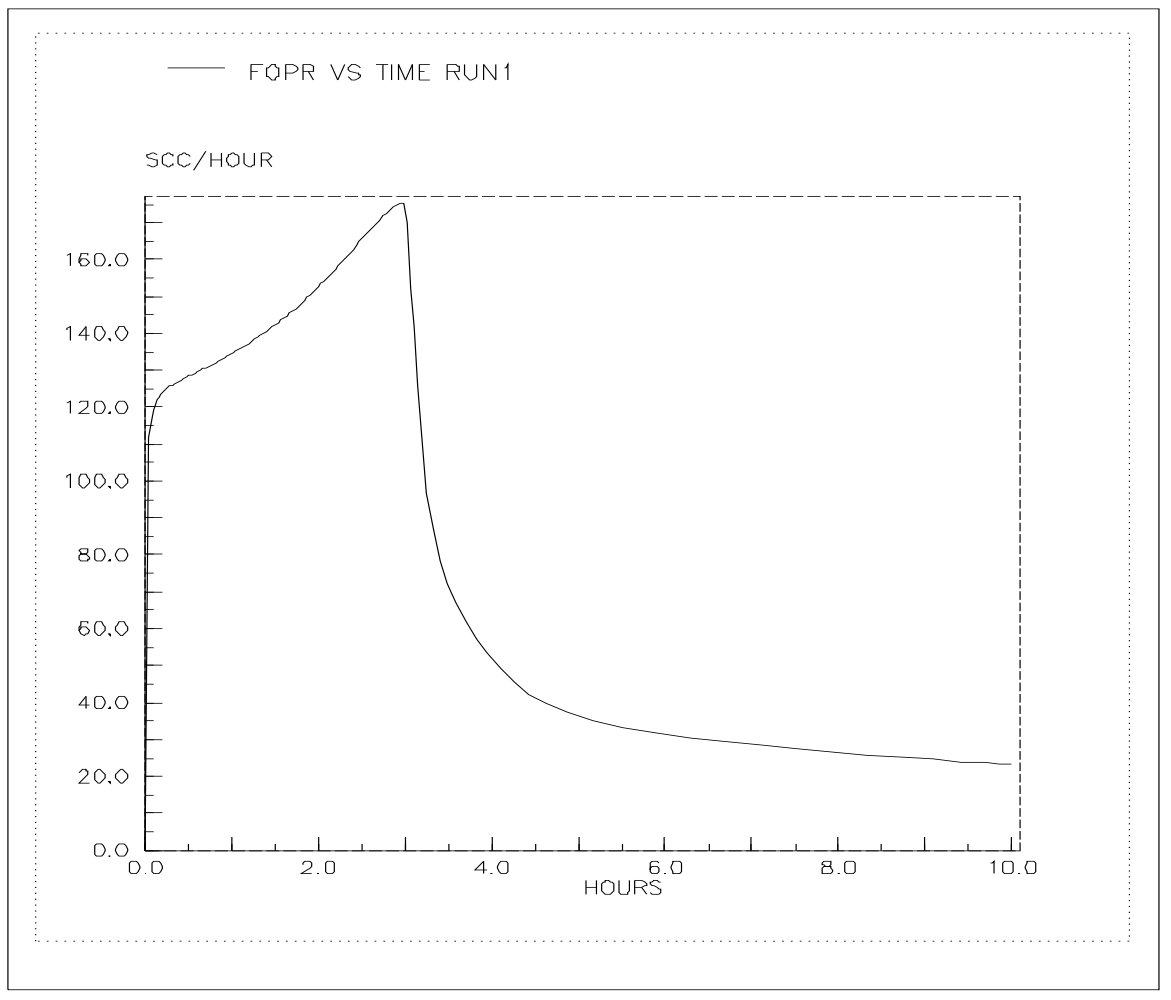

Figure 8.6: Oil Production Rate for Vertical Injector and Producer

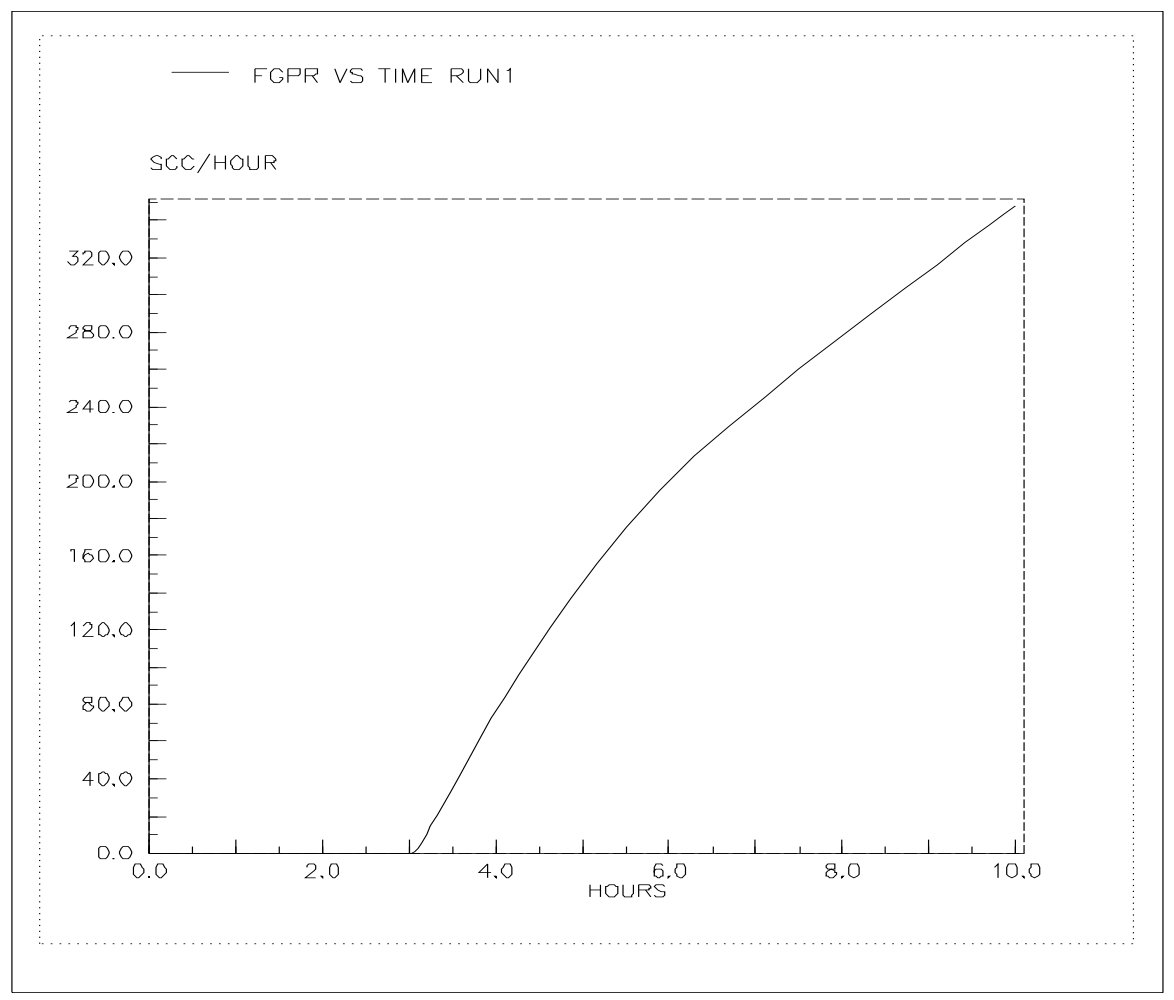

Figure 8.7: Gas Production Rate for Vertical Injector and Producer 


\section{Reference}

1. Meszaros, G., Chakma, A., and Islam, M.R., "Scaled Model Studies and Numerical Simulation of Inert Gas Injection with Horizontal Wells", SPE 20529, 65th annual technical conference and exhibition, New Orleans, LA, Sept 23-26, 1990, pg 585.

2. Dykstra, H., and Dickinson, W.,"Oil Recovery by Gravity Drainage into Horizontal Wells Compared with Recovery from Vertical Wells", SPE 19827, 64th anual technical conference, San Antonio, TX, Oct 8-11, 1989, pg 607.

3. Williams, Richard Jr., "The effects of horizontal well placement and gravity on sweep efficiency", Ch 4, M.S. Thesis,1997, pg 23.

4. Nasr, T.N., Kimber, K.D., Vendrinsky, D.A., and Jha, K.N.,"Process Enhancement in Horizontal Wells through the Use of Vertical Drainage Channels and Hydrocarbon Additives", SPE 21794, Western regional meeting, Long Beach, CA, Mar 20-22, 1991, $\operatorname{pg} 417$.

5. Jha, K.N., and Chakma, A., "Nitrogen Injection with Horizontal Wells for Enhancing Heavy Oil Recovery:2-D and 3-D models", SPE 23029, Asia-pacific conference, Perth, Australia, Nov 4-7, 1991, pg 765.

6. Edmunds, N.R., and Sugget, J.C., "Design of a Commercial SAGD Heavy Oil Project", SPE 30277, Heavy Oil symposium, Calgary, Canada,19-21 june 1995, pg 287.

7. Ahner, P.F., and Sufi, A.H.,"Physical Model Steamflood Studies Using Horizontal Wells", SPE 20247, SPE Reservoir Engineering, Feb. 1994, pg 59.

8. Zhou, D., Fayers, F.J., and Orr, F.M. Jr., "Scaling of Multiphase Flow in Simple Heterogeneous Porous Media", SPE 27833, pg 559. 


\title{
9. Correlations for Cresting Behavior in Horizontal Wells
}

\author{
by Antonio Souza, Sepehr Arbabi, and Khalid Aziz
}

\begin{abstract}
Water and gas cresting in horizontal wells are important phenomena in reservoirs that have an aquifer and/or a gas-cap. In practical situations, many reservoirs are produced under supercritical rates and a breakthrough of the displacing phase becomes inevitable. At the beginning of a reservoir simulation study, it is a common procedure to make an estimate of the breakthrough time and the post-breakthrough behavior. Grid sensitivity runs are also made to obtain the best grid block sizes to use, since an accurate representation of cresting behavior requires fine grid simulations, which are costly and not always practical. In this work a procedure was developed to obtain accurate breakthrough times using just coarse grid simulations. The flow equations were written in dimensionless form and important parameters affecting multiphase flow were identified. Simple correlations for a quick estimate of breakthrough time, maximum oil rate and post-breakthrough behavior were derived based on an appropriate set of dimensionless variables and an extensive number of simulation runs. The effects of gridblock size and grid pattern were investigated in detail. Effects of rate, mobility ratio, well drainage area, well height, and end-points and shapes of relative permeability curves were also included. A procedure to derive pseudofunctions either using numerical correlations or coarse grid simulations is also presented. These pseudofunctions can be used to improve the performance of coarse grid simulations. An optimum grid pattern to start a reservoir simulation study is proposed. Results for fields with several horizontal wells are also discussed. Application to a real field example shows that the correlations and procedures derived are reliable and accurate, and can be used for field situations before starting a reservoir simulation study.
\end{abstract}

\subsection{Introduction}

Petroleum reservoirs often have a gas cap and/or an aquifer. In these situations they are subjected to rapid gas and/or water movement towards the well, created by a sharp pressure gradient in the well direction. As the production begins, the interface between the fluids, that is, gas-oil contact or water-oil contact, deforms from its initial plane shape to a cone or crest. When a field is developed by vertical wells, the shape of the deformation is called a cone, but when it is developed by horizontal wells, this deformation is better described as a crest.

Figure 9.1 shows the general three phase model of the cresting behavior, with the two crests approaching the well as the production continues.

Several analytical solutions for coning behavior for vertical wells and more recently

for horizontal wells have been reported in the literature $[1,2,3,4,5,6,7]$. Most of those solutions were developed for the steady-state condition reached by the cone with a constant potential boundary condition at the lateral boundaries of the reservoir. This is normally 


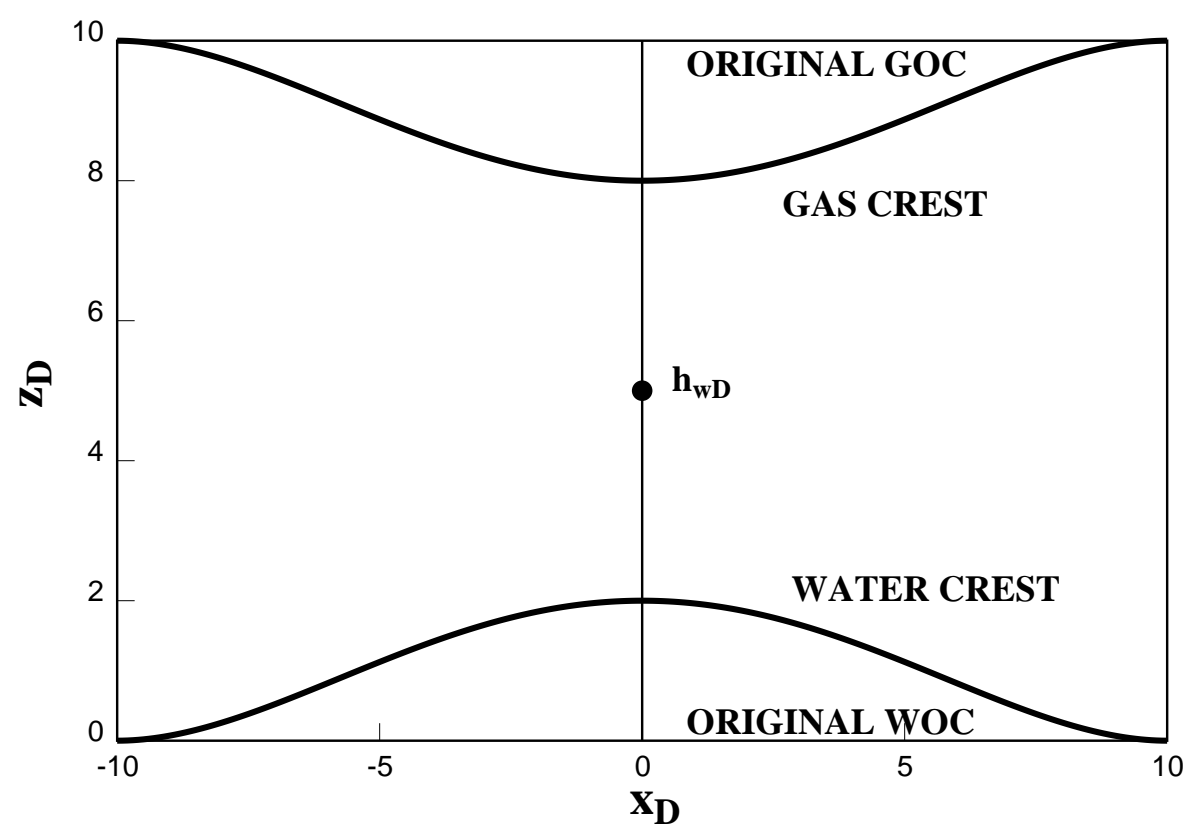

Figure 9.1: General model for cresting behavior

done to calculate the critical rate, for which several different approaches have been used. These solutions are useful when the injection pattern in the reservoir creates a constant potential boundary between production and injection wells.

There are also analytical solutions available for infinite-acting reservoirs and for closed-boundary reservoirs, the latter being specially useful when there are several production wells in a line, creating a no-flow boundary $[8,9,10,11,12]$. In those situations, it becomes important to know the breakthrough time of the displacing phase (gas or water) and the post-breakthrough behavior for supercritical rates.

Analytical solutions are useful for several cases shown in the literature, since they can give a good estimate, are easy to apply and, more importantly, they do work for some field situations. For these reasons, despite their limitations, they are still being developed and used, at least during the early stages of a reservoir study.

The assumptions used to derive analytical solutions limit their applicability. These limitations have led to the use of numerical simulators to study coning and cresting behavior. Simulators solve the complete set of equations, along with the correct boundary conditions, they can handle important reservoir complexities, and their use has become a standard practice in reservoir engineering.

However, simulators have their own limitations. The numerical diffusion created by the approximations made during the discretization of time and spatial terms leads to the requirement of using a large number of blocks to achieve accurate results. The number of blocks are limited by computer storage and CPU time consideration which are ultimately limited by hardware and economic constraints.

New correlations are developed here for breakthrough time and post-breakthrough behavior of horizontal wells. A procedure to obtain an accurate breakthrough time using just coarse grid simulations is also proposed. 


\subsection{Assumptions and Dimensionless Equations}

Correlations are more general and reliable when derived based on the fundamental flow equations, so that the important physical mechanisms are automatically incorporated in the correlations. The flow equations, however, have a set of dimensional variables that, with some assumptions, can be easily incorporated in dimensionless variables, reducing the number of variables to be used to develop correlations. We first state the assumptions used in this study. The main assumptions are:

1. Homogeneous medium (constant permeability in each direction).

2. Non-deformable formation $\left(c_{r c}=0\right)$ and constant porosity.

3. Isotropic medium (same permeability all directions).

4. Constant viscosity for each phase.

5. Absence of capillary pressures.

6. Two-dimensional flow : For vertical wells, this implies radial flow and the problem is solved in r-z coordinates. For horizontal wells, this implies a cross-sectional model, with the well penetrating the entire formation. In this situation, the pressure drop in the well is also neglected.

7. Undersaturated reservoir $\left(p>p_{b p}\right)$.

8. Constant production rate.

9. Two-phase flow.

10. Symmetry for the well position : Assume that the lateral boundary size is the same for both sides of the well.

For this study, the following set of dimensionless variables can be defined:

1. Lateral dimensions:

$$
x_{D}=\frac{x}{h_{o}} \sqrt{\left(\frac{k_{v}}{k_{h}}\right)}
$$

2. Vertical dimension:

$$
\begin{gathered}
z_{D}=\frac{z}{h_{o}} \\
\lambda_{o}=\frac{k_{r o c w}}{\mu_{o}}
\end{gathered}
$$


3. Potential:

$$
\Phi_{D}=\frac{\Phi}{\Delta \rho g h_{o}}
$$

4. Well drainage distance: using Equation 9.1:

$$
x_{e D}=\frac{x_{e}}{h_{o}} \sqrt{\left(\frac{k_{v}}{k_{h}}\right)}
$$

5. Well height:

$$
h_{w D}=\frac{h_{w}}{h_{o}}
$$

6. Mobility ratio:

$$
M=\frac{k_{\text {rwro }}}{k_{\text {rocw }}} \frac{\mu_{o}}{\mu_{w}}
$$

7. Dimensionless time

$$
t_{D}=\frac{\Delta \rho g \lambda_{o} k_{v} t}{\phi_{e f} h_{o}}
$$

where $\Delta \rho$ is the initial density difference at water-oil contact at reservoir conditions, and is given by:

$$
\Delta \rho=\rho_{w}-\rho_{o}
$$

with

$$
\rho_{w}=\frac{\rho_{w s t d}}{B_{w}}
$$

and

$$
\rho_{o}=\frac{\rho_{o s t d}+\rho_{g s t d} R_{s}}{B_{o}}
$$

The oil mobility $\lambda$ is given by Equation 9.3

8. Dimensionless rate

$$
q_{D}=\frac{q_{T}}{2 \pi \Delta \rho g \lambda_{o} \sqrt{k_{v} k_{h}} L h_{o}}
$$

where $q_{T}$ is the total rate at reservoir conditions. 
9. Dimensionless cumulative oil recovery

$$
N_{p D}=\frac{N_{p} \sqrt{\frac{k_{v}}{k_{h}}}}{2 \pi \phi_{e f} L h_{o}{ }^{2}}
$$

10. Dimensionless pressure

$$
p_{D}=\frac{p-p_{S T D}}{\Delta \rho g h_{o}}
$$

11. Dimensionless compressibility

$$
c_{l D}=c_{l} \Delta \rho g h_{o}, \quad l=o, w
$$

12. Normalized saturations

$$
\begin{aligned}
& S_{w}^{*}=\frac{S_{w}-S_{w c}}{1-S_{w c}-S_{o r}} \\
& S_{o}^{*}=\frac{S_{o}-S_{o r}}{1-S_{w c}-S_{o r}}
\end{aligned}
$$

so that

$$
S_{o}^{*}+S_{w}^{*}=1
$$

13. Normalized relative permeabilities

Relative permeabilities can be written as

$$
\begin{gathered}
k_{r w}=k_{r w r o} k_{r w}{ }^{*} \\
k_{r o}=k_{r o c w} k_{r o}{ }^{*}
\end{gathered}
$$

The normalized relative permeabilities $k_{r w}^{*}$ and $k_{r o}^{*}$ are given by power functions of saturations as

$$
\begin{gathered}
k_{r w}^{*}=\left(S_{w}^{*}\right)^{n_{w}} \\
k_{r o}^{*}=\left(S_{o}^{*}\right)^{n_{o}}
\end{gathered}
$$


Using this set of dimensionless variables, the dimensionless equations for oil and water can be written as, [13]

Oil:

$$
\frac{\partial}{\partial x_{D}}\left[\frac{k_{r o}^{*}}{B_{o}} \frac{\partial \Phi_{o D}}{\partial x_{D}}\right]+\frac{\partial}{\partial z_{D}}\left[\frac{k_{r o}^{*}}{B_{o}} \frac{\partial \Phi_{o D}}{\partial z_{D}}\right]-\frac{2 \pi q_{o D}}{B_{o}}=\frac{\partial}{\partial t_{D}}\left(\frac{S_{o}^{*}}{B_{o}}\right)
$$

Water:

$$
\frac{\partial}{\partial x_{D}}\left[\frac{k_{r w}^{*}}{B_{w}} \frac{\partial \Phi_{w D}}{\partial x_{D}}\right]+\frac{\partial}{\partial z_{D}}\left[\frac{k_{r o}^{*}}{B_{w}} \frac{\partial \Phi_{w D}}{\partial z_{D}}\right]-\frac{2 \pi q_{w D}}{B_{w}}=\frac{1}{M} \frac{\partial}{\partial t_{D}}\left(\frac{S_{w}^{*}}{B_{w}}\right)
$$

Where:

$$
q_{l D}=\frac{q_{l}}{2 \pi \Delta \rho g \lambda_{l} \sqrt{k_{v} k_{h}} L h_{o}}, \quad l=o, w
$$

or

$$
q_{l D}=\frac{q_{D} k_{r l}^{*}}{k_{r o}^{*}+M k_{r w}^{*}}, \quad l=o, w
$$

and

$$
q_{o D}+M q_{w D}=q_{D}
$$

The mobilities are given by:

$$
\lambda_{l}=\frac{k_{\text {rwro }}}{\mu_{w}}
$$

These dimensionless equations show the important dimensionless variables that should be used to develop correlations for water cresting behavior. Those dimensionless variables are $t_{D}, q_{D}, \mathrm{M}$ and two dimensionless variables relating the shape of the relative permeabilities curves, which are chosen here as the exponents $n_{o}$ and $n_{w}$. The dimensionless variable $h_{w D}$ relating the location of the well given by Equation 9.6 and the well drainage distance given by Equation 9.5 are also used. The compressibility effects of $B_{o}$ and $B_{w}$ are given by Equations 9.10 and 9.11, which also consider the effect of a constant $R_{s}$ for undersaturated reservoir.

The boundary conditions are no-flow on the lateral edge and on the top of the reservoir. A large aquifer to give pressure support is placed at the bottom of the reservoir.

An aquifer size of 200 times the reservoir size must be used to maintain the reservoir pressure without any injection. Smaller aquifers result in rapid decline of reservoir pressure, which normally results in changing the well control from constant production rate to specified bottom hole pressure. This completely changes the breakthrough time, since the assumption of constant production rate is no longer valid.

A large aquifer, however, can also be modeled with the same results using a line of injectors at the bottom of the aquifer with total injection matching total production, for an aquifer size of 20 times the reservoir size. This line of injectors was simulated here using a horizontal well injector in the transverse direction.

ALso, in order to avoid high temporal numerical diffusion effects, the dimensionless time-step size should not be larger than one hundredth of the dimensionless breakthrough time $\left(\Delta t_{D}=0.01 t_{b t D}\right)$. 


\subsection{Simulations}

Equations 9.23 and 9.24 derived in Section 9.2 can be solved using a commercial simulator with the appropriate input data. The ECLIPSE 100 simulator [14], version 95a, was used for the simulations.

Figure 9.2 shows an example of the grid used to make the runs. The horizontal producer is represented by the point close to the top of the reservoir, drilled in the orthogonal direction $\mathrm{y}$ of the plane $\mathrm{xz}$. The horizontal injector crosses the entire reservoir in the $\mathrm{x}$ direction and is located at the bottom of the aquifer.

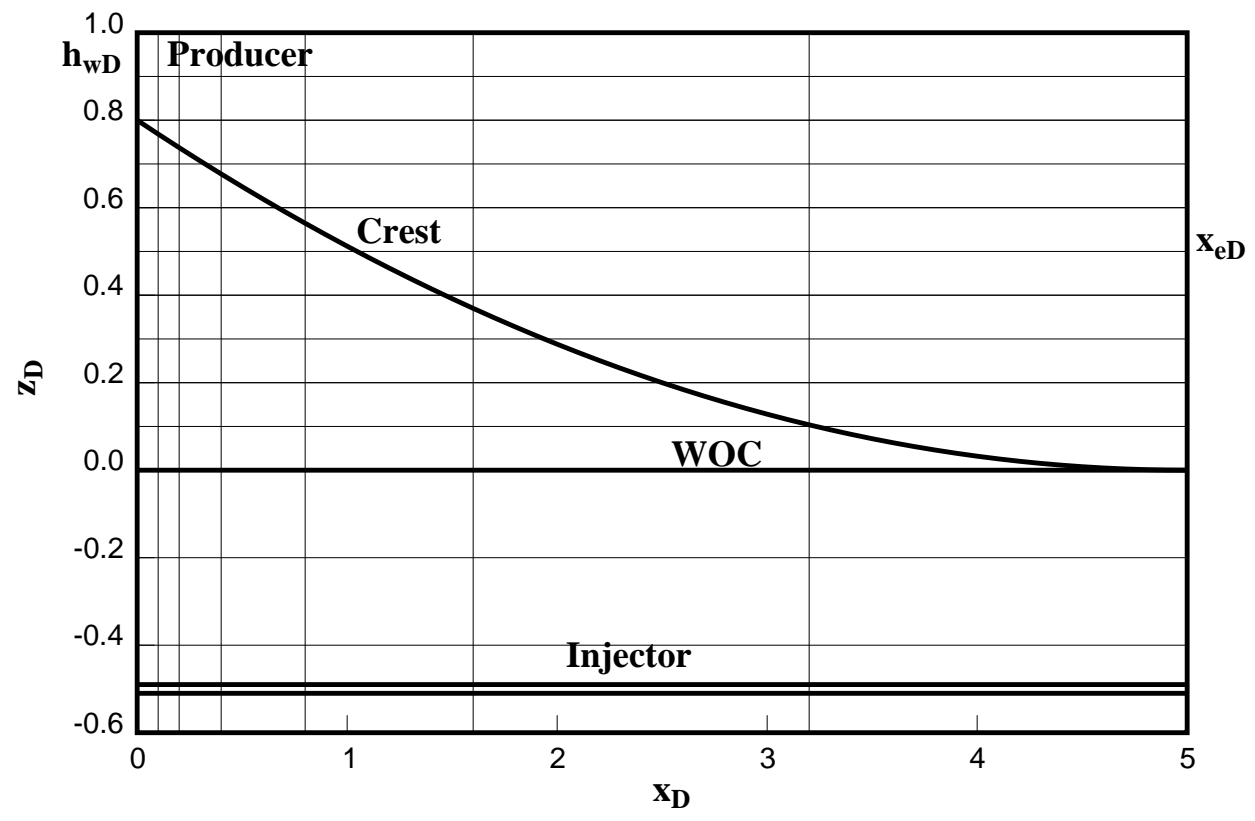

Figure 9.2: Grid example for the simulations.

Since the main idea of the work is to derive correlations for breakthrough time and post-breakthrough behavior as functions of the gridblock sizes and the dimensionless variables described in the last section, several initial runs were performed in order to verify their sensitivities. This was done by varying horizontal and vertical gridblock sizes, lateral boundary size, aquifer size, time-step size and the dimensionless variables. The procedures used and initial conclusions reached are described next.

\subsubsection{Grid Block Size}

An initial set of runs demonstrated that breakthrough time and WOR are more affected by vertical gridblock size dimensions than by horizontal ones. This was expected since the crest moves from the water-oil contact to the well mainly in the vertical direction, so that the numerical diffusion effect in this direction is higher than in the horizontal one.

Assuming a constant horizontal pattern, a plot of the dimensionless breakthrough time $\left(t_{b t D}\right)$ against the number of blocks used in the vertical direction $\left(n_{b z}\right)$ gives the behavior shown in Figure 9.3. This curve suggests an asymptotic shape tending to a value of $t_{b t D}^{0}$, 
which would represent the dimensionless breakthrough time when the number of blocks in the vertical direction tends to infinity.

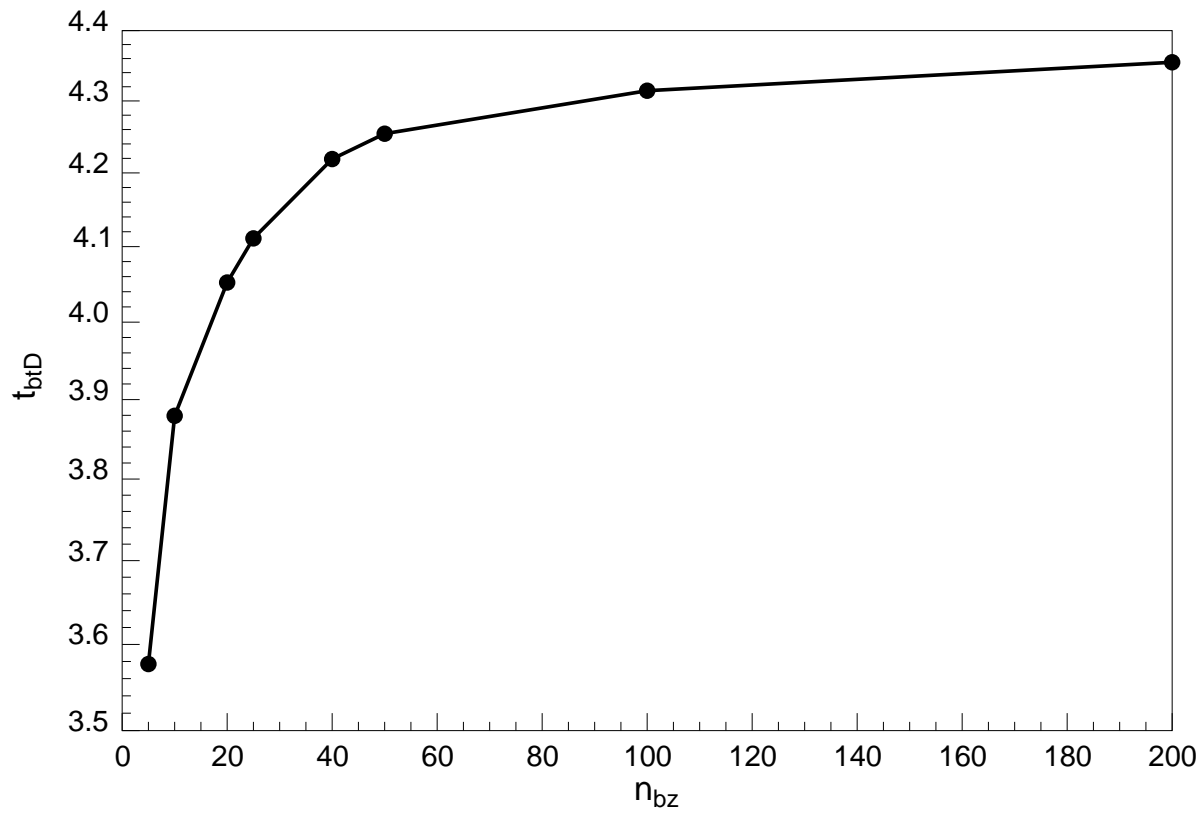

Figure 9.3: Effect of number of blocks used between the top of the formation and water-oil contact on breakthrough time.

One of the simplest functions that can approximate this behavior is the following:

$$
t_{b t D}=t_{b t D}^{0} e^{-\frac{\alpha}{n_{b z}}}
$$

If the vertical gridblock size remains constant throughout the oil column, $\Delta z_{D}$ will be the inverse of $n_{b z}$ and Equation 9.29 can be written as

$$
t_{b t D}=t_{b t D}^{0} e^{-\alpha \Delta z_{D}}
$$

which gives a straight line in a plot of $\ln t_{b t D}$ vs $\Delta z_{D}$. The parameters $t_{b t D}^{0}$ and $\alpha$ are functions of the dimensionless variables described earlier. Two assumptions, however, must be made to write the correlations in such a way: a constant vertical gridblock size for each simulation and a horizontal grid pattern that minimizes the effects of the gridblock sizes on flow in that direction. These assumptions are analyzed next.

\subsubsection{Vertical Gridblock Size Analysis}

In the vertical direction, uniform grid with refinement close to the well works best. We found that $\Delta Z_{D}=2 \times 0.01,0.02,2 \times 0.03,9 \times 0.1$ was almost as good as a uniform grid of $\Delta Z_{D}=0.01$.

\subsubsection{Horizontal Gridblock Size Analysis}

In the horizontal direction, the suggested pattern is to use the same size (smallest gridblock size in the vertical direction) for the first two blocks and then start doubling the 


\begin{tabular}{|l|l|}
\hline Oil column, $h$ & $100 \mathrm{~m}$ \\
\hline Horizontal permeability, $k_{h}$ & $1 \mathrm{~d}$ \\
\hline Vertical permeability, $k_{v}$ & $1 \mathrm{~d}$ \\
\hline Porosity, $\phi$ & 0.1 \\
\hline Oil density, $\rho_{o}$ (standard) & $1000 \mathrm{~kg} / \mathrm{m}^{3}$ \\
\hline Water density, $\rho_{w}$ (standard) & $900 \mathrm{~kg} / \mathrm{m}^{3}$ \\
\hline Gas density, $\rho_{g}$ (standard) & $1 \mathrm{~kg} / \mathrm{m}^{3}$ \\
\hline Gas-oil solubility ratio $R s$ & 0 \\
\hline Oil viscosity, $\mu_{o}$ & $1 \mathrm{cp}$ \\
\hline Water viscosity, $\mu_{w}$ & $1 \mathrm{cp}$ \\
\hline Oil compressibility, $c_{o}$ & $1 \mathrm{e}-5 \mathrm{bar}^{-1}$ \\
\hline Water compressibility, $c_{w}$ & $1 \mathrm{e}-5 \mathrm{bar}^{-1}$ \\
\hline Rock compressibility, $c_{r}$ & 0.0 \\
\hline Connate water saturation, $S_{w c}$ & 0 \\
\hline Residual oil saturation, $S_{o r}$ & 0 \\
\hline Oil relative permeability exponent, $n_{o}$ & 1 \\
\hline Water relative permeability exponent, $n_{w}$ & 1 \\
\hline Oil relative permeability at $S_{w c}, k_{r o c w}$ & 1 \\
\hline Water relative permeability at $S_{o r}, k_{r w r o}$ & 1 \\
\hline Production rate, $q\left(q_{D}=0.1\right)$ & $5428.74 \mathrm{~m}^{3} / \mathrm{day}$ \\
\hline
\end{tabular}

Table 9.1: Base case data set for simulation runs

gridblock size up to the lateral boundary. For example, if the smallest vertical gridblock size is 0.01 , then the horizontal gridblock sizes are $\Delta x_{D}=0.01,0.01,0.02,0.04,0.08,0.16, \ldots$. The size of the last block is adjusted for the location of the lateral boundary. The results based on this pattern were compared with four other patterns and only minor differences were seen. However, the proposed pattern has the advantage that it gives the best approach to a straight line and therefore gives the best match with Equation 9.30.

\subsection{Water Cresting Correlations}

The base case for the runs was defined for the situation when the reservoir behaves as infinite, with mobility equal to one. The well is at the top of the reservoir and relative permeability curves are straight lines. The input data for the ECLIPSE simulator [14] for the base case are shown in Table 9.1.

From this base case, each dimensionless variable was varied over a large range of practical situations, as shown in Table 9.2. For example, the dimensionless rate varies from 0.005 to 1.0, which using the ECLIPSE data shown in Table 9.1, corresponds to a variation in the dimensional rate from $250 \mathrm{~m}^{3} /$ day to $50000 \mathrm{~m}^{3} /$ day.

For each value of dimensionless variable, six vertical gridblock size $\Delta z_{D}$ were used, with $n_{b z}$ varying from 20 to 200 , covering the vertical range for most of the practical simulations. Table 9.3 shows this range, with the horizontal gridblock sizes following the pattern 


\begin{tabular}{|c|c|c|}
\hline DIMENSIONLESS VARIABLE & BASE CASE & VARIATION \\
\hline$q_{D}$ & 0.1 & 0.005 to 1 \\
\hline$M$ & 1 & 0.1 to 100 \\
\hline$x_{e D}$ & 20 & 2.5 to 20 \\
\hline$h_{w D}$ & 1 & 0.2 to 1 \\
\hline$n_{o}$ & 1 & 1 to 7 \\
\hline$n_{w}$ & 1 & 1 to 3 \\
\hline
\end{tabular}

Table 9.2: Base case for the simulations and range of variation of each dimensionless variable.

\begin{tabular}{|c|c|r|c|}
\hline$\Delta z_{D}$ & $n_{b z}$ & $\Delta x_{D}$ & $n_{b x}$ \\
\hline 0.005 & 200 & $0.005,0.005,0.01,0.02,0.04,0.08,0.16, \ldots, 5.12,9.76$ & 13 \\
\hline 0.01 & 100 & $0.01,0.01,0.02,0.04,0.08,0.16, \ldots, 5.12,9.76$ & 12 \\
\hline 0.02 & 50 & $0.02,0.02,0.04,0.08,0.16, \ldots, 5.12,9.76$ & 11 \\
\hline 0.025 & 40 & $0.02,0.02,0.04,0.08,0.16, \ldots, 5.12,9.76$ & 11 \\
\hline 0.04 & 25 & $0.04,0.04,0.08,0.16, \ldots, 5.12,9.76$ & 10 \\
\hline 0.05 & 20 & $0.04,0.04,0.08,0.16, \ldots, 5.12,9.76$ & 10 \\
\hline
\end{tabular}

Table 9.3: Grid patterns for the simulations.

suggested in the last chapter.

The next sections describe the specific procedures used to obtain the correlations. It took about 5000 runs with the ECLIPSE simulator [14] to obtain the results of this work. Each run varied from 1 minute to 4 hours of CPU time in an alpha machine, with a total of around 3000 CPU hours.

\subsubsection{Breakthrough Time}

The correlations for $t_{b t D}$ were derived using Equation 9.30, which can be rewritten as:

$$
\ln \frac{t_{b t D}^{0}}{t_{b t D}}=\alpha \Delta z_{D}
$$

which means that $\ln t_{b t D}$ is a linear function of $\Delta z_{D}$, with $t_{b t D}^{0}$ and $\alpha$ being the parameters to be found.

The procedure used was to record the values of breakthrough time for each run, defined to be the time to reach a water-cut of 0.001 . Then, a linear regression with the six points of $\Delta z_{D}$ and $t_{b t D}$ was performed, using Equation 9.31. Figure 9.4 shows one example of such a match, for $q_{D}=0.5$.

With this procedure, for each dimensionless variable, the values of $t_{b t D}^{0}$ and $\alpha$ were obtained. These values are shown in Table 9.4, together with parameter $m$ for postbreakthrough behavior defined in Section 9.4.2. 


\begin{tabular}{|c|c|c|c|c|c|c|c|c|}
\hline$q_{D}$ & $\mathrm{M}$ & $x_{e D}$ & $h_{w D}$ & $n_{o}$ & $n_{w}$ & $t_{b t D}^{0}$ & $\alpha$ & $m$ \\
\hline 0.1 & 1 & 20 & 1 & 1 & 1 & 4.3889 & 1.6048 & 0.5170 \\
\hline 0.15 & & & & & & 2.4227 & 2.3122 & 0.5058 \\
\hline 0.2 & & & & & & 1.6310 & 2.9727 & 0.5000 \\
\hline 0.3 & & & & & & 0.9653 & 4.0448 & 0.4866 \\
\hline 0.5 & & & & & & 0.5243 & 6.9362 & 0.4678 \\
\hline 0.7 & & & & & & 0.3445 & 9.0971 & 0.4447 \\
\hline 1.0 & & & & & & 0.2300 & 11.1761 & 0.4366 \\
\hline 0.07 & & & & & & 7.7275 & 1.2328 & 0.5353 \\
\hline 0.05 & & & & & & 13.5931 & 0.8270 & 0.5576 \\
\hline 0.03 & & & & & & 33.9145 & 0.5843 & 0.6013 \\
\hline 0.02 & & & & & & 72.0729 & 0.4114 & 0.6531 \\
\hline 0.01 & & & & & & 254.285 & 0.1537 & 0.9917 \\
\hline 0.005 & & & & & & 729.435 & 0.1444 & 1.6030 \\
\hline & 2 & & & & & 3.6525 & 1.5111 & 0.5538 \\
\hline & 5 & & & & & 3.1642 & 1.5884 & 0.5897 \\
\hline & 10 & & & & & 2.9799 & 1.4732 & 0.6094 \\
\hline & 50 & & & & & 2.8323 & 1.5454 & 0.6335 \\
\hline & 100 & & & & & 2.8003 & 1.4203 & 0.6371 \\
\hline & 0.5 & & & & & 5.6395 & 1.3949 & 0.4745 \\
\hline & 0.1 & & & & & 12.6440 & 1.3741 & 0.4062 \\
\hline & & 10 & & & & 4.3514 & 1.6373 & 0.5329 \\
\hline & & 5 & & & & 4.1576 & 1.5188 & 0.6129 \\
\hline & & 2.5 & & & & 3.5721 & 1.6658 & 0.9256 \\
\hline & & & 0.8 & & & 3.5447 & 2.4520 & 0.4451 \\
\hline & & & 0.6 & & & 2.2080 & 3.5495 & 0.3634 \\
\hline & & & 0.4 & & & 0.9943 & 6.7327 & 0.2715 \\
\hline & & & 0.2 & & & 0.2495 & 29.6500 & 0.2074 \\
\hline & & & & 2 & & 4.1237 & 2.1519 & 0.4938 \\
\hline & & & & 3 & & 3.6852 & 2.6456 & 0.4668 \\
\hline & & & & 4 & & 3.2572 & 2.8951 & 0.4492 \\
\hline & & & & 5 & & 2.9260 & 3.2076 & 0.4410 \\
\hline & & & & 6 & & 2.6515 & 3.0973 & 0.4355 \\
\hline & & & & & 2 & 4.4433 & 0.7300 & 0.5196 \\
\hline & & & & & 3 & 4.4626 & 0.3964 & 0.5201 \\
\hline
\end{tabular}

Table 9.4: Values of $t_{b t D}^{0}, \alpha$ for breakthrough time and $m$ for $q_{o D}$ for each dimensionless variable simulated. A blank entry indicates that dimensionless variable has its base value (first row). 


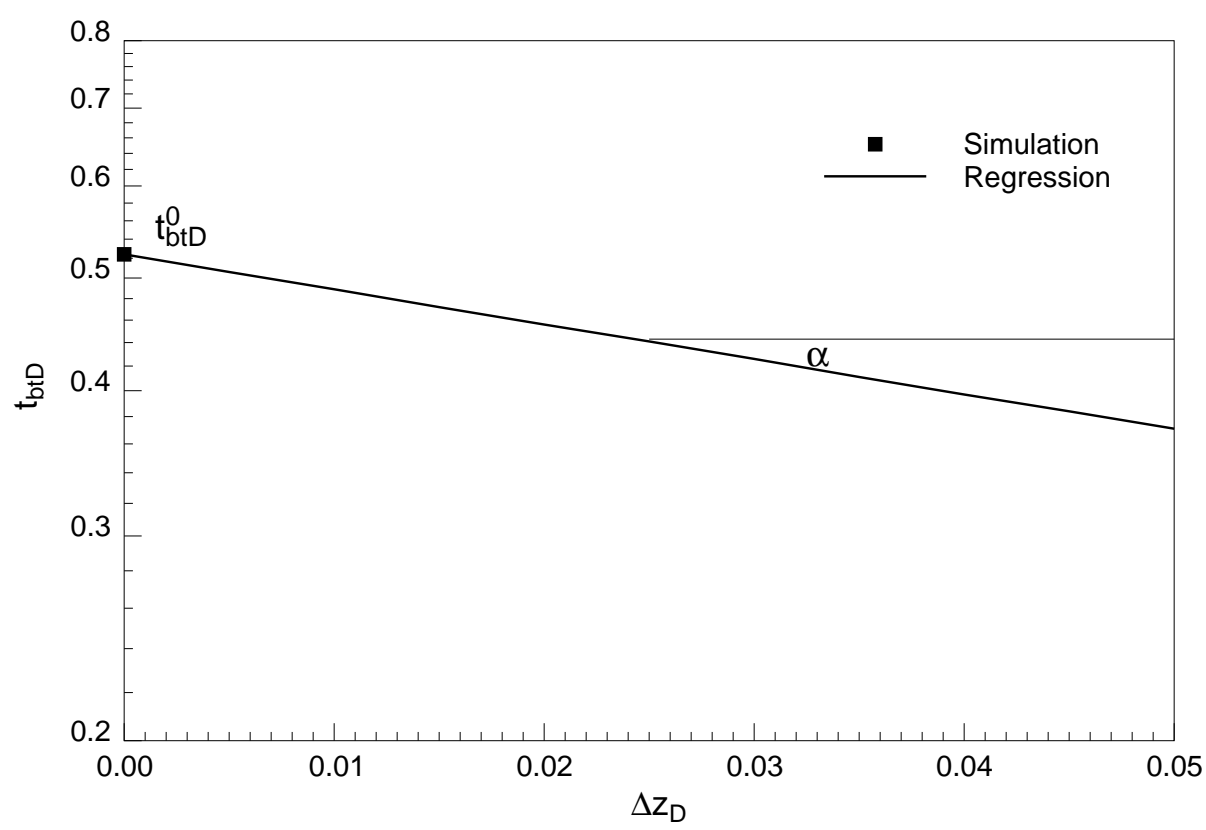

Figure 9.4: Linear regression of $t_{b t D}$ vs $\Delta z_{D}$ for $q_{D}=0.5$

Next, the correlations for the parameters $t_{b t D}^{0}$ and $\alpha$ as functions of the dimensionless variables were developed. The variations in dimensionless variables were made from the base case, that is, only one dimensionless variable was varied for each set of $\Delta z_{D}$. It was assumed that each dimensionless variable could be matched independently, so that the final correlation is a combination of several functions of just one variable. This assumption implies that the effect of simultaneous variations in two or more dimensionless variables can be calculated as a combination of separate variations in each dimensionless variable from the base case. The runs showed that this is a good assumption for most combinations of dimensionless variables except for simultaneous variations of $q_{D}$ with $M, x_{e D}$ or $n_{o}$. For this reason, an extra set of runs varying these dimensionless variables was made and the values of $t_{b t D}^{0}$ and $\alpha$ for these runs are shown in Tables 9.5 and 9.6. Extra functions of these dimensionless variables were attached to each correlation to account for this.

The values of $q_{D}$ were matched with each value of $t_{b t D}^{0}$ and $\alpha$. The other parameters were matched as correction factors, that is, the ratio between each value and its correspondent base case value. For example, each value of $t_{b t D}^{0}$ for the well location $h_{w D}=0.8,0.6,0.4$ and 0.2 was divided by 4.3889 (see Table 9.4), which is the value of $t_{b t D}^{0}$ for the base case. These ratios were then used in the regression with the $h_{w D}$ values. In this way, any dimensionless variable in the following correlations, except for $q_{D}$, can be skipped for a quick estimate of breakthrough time, if this dimensionless variable has its base case value. For example, the base case value for mobility ratio is $M=1$. If this value can be assumed for a real field situation, the factors for $\mathrm{M}$ in Equations 9.34 and 9.35 are equal to one and can be skipped from the correlations.

It could also be seen that in some situations a dimensionless variable has a small effect on results. In these situations, the dimensionless variable was not considered in the correlations.

A combination of linear regression and the polytope procedure [15] was used for 


\begin{tabular}{|c|c|c|c|c|c|}
\hline$q_{D}$ & $\mathrm{M}$ & $x_{e D}$ & $t_{b t D}^{0}$ & $\alpha$ & $m$ \\
\hline 0.2 & 2 & & 1.2476 & 3.2477 & 0.5481 \\
\hline 0.2 & 5 & & 0.9673 & 3.8930 & 0.6008 \\
\hline 0.2 & 10 & & 0.8432 & 4.4230 & 0.6119 \\
\hline 0.2 & 50 & & 0.7190 & 4.8453 & 0.6385 \\
\hline 0.2 & 0.5 & & 2.2655 & 2.2006 & 0.4498 \\
\hline 0.5 & 2 & & 0.3388 & 11.8824 & 0.4356 \\
\hline 0.5 & 5 & & 1.1401 & 14.0348 & 0.0744 \\
\hline 0.5 & 0.5 & & 0.7745 & 4.0770 & 0.4309 \\
\hline 0.05 & 2 & & 12.2626 & 0.8370 & 0.6038 \\
\hline 0.05 & 5 & & 11.3972 & 0.8720 & 0.6405 \\
\hline 0.05 & 10 & & 11.0968 & 0.8493 & 0.6559 \\
\hline 0.05 & 50 & & 10.8592 & 0.8819 & 0.6733 \\
\hline 0.05 & 100 & & 10.8220 & 0.8669 & 0.6789 \\
\hline 0.05 & 0.5 & & 16.0048 & 0.7757 & 0.5154 \\
\hline 0.02 & 2 & & 69.6013 & 0.5326 & 0.6697 \\
\hline 0.02 & 5 & & 68.0938 & 0.6324 & 0.6849 \\
\hline 0.02 & 10 & & 67.5586 & 0.6661 & 0.6922 \\
\hline 0.02 & 50 & & 67.0587 & 0.5439 & 0.6938 \\
\hline 0.02 & 0.5 & & 76.7792 & 0.3609 & 0.6321 \\
\hline 0.2 & & 10 & 1.6250 & 2.9871 & 0.5081 \\
\hline 0.2 & & 5 & 1.5842 & 3.0518 & 0.5499 \\
\hline 0.2 & & 2.5 & 1.4496 & 3.3277 & 0.7285 \\
\hline 0.5 & & 10 & 0.5196 & 6.7655 & 0.4730 \\
\hline 0.5 & & 5 & 0.5137 & 7.0519 & 0.5011 \\
\hline 0.5 & & 2.5 & 0.4815 & 7.4504 & 0.6114 \\
\hline 0.05 & & 10 & 13.3166 & 0.7036 & 0.5953 \\
\hline 0.05 & & 5 & 12.1040 & 0.7787 & 0.8287 \\
\hline 0.05 & & 2.5 & 9.0261 & 0.8581 & 1.3244 \\
\hline 0.02 & & 10 & 64.7916 & 0.1272 & 0.9707 \\
\hline 0.02 & & 5 & 46.3915 & 0.2128 & 1.4986 \\
\hline 0.02 & & 2.5 & 28.2599 & 0.2392 & 2.1657 \\
\hline 0.01 & & 10 & 183.151 & 0.1444 & 1.5506 \\
\hline 0.01 & & 5 & 111.778 & 0.1945 & 2.2586 \\
\hline 0.01 & & 2.5 & 62.973 & 0.1296 & 3.2044 \\
\hline
\end{tabular}

Table 9.5: Values of $t_{b t D}^{0}, \alpha$ for breakthrough time and $m$ for $q_{o D}$ for simultaneous variations of $q_{D}$ with $M$ or $x_{e D}$. $h_{w D}, n_{o}$ and $n_{w}$ are all equal to 1 , their base values. Also a blank entry indicates that dimensionless variable has its base value. 


\begin{tabular}{|c|c|c|c|c|}
\hline$q_{D}$ & $n_{o}$ & $t_{b t D}^{0}$ & $\alpha$ & $m$ \\
\hline 0.2 & 2 & 1.4872 & 4.0277 & 0.4711 \\
\hline 0.2 & 4 & 1.1382 & 4.3968 & 0.4215 \\
\hline 0.2 & 6 & 0.9393 & 4.4910 & 0.4153 \\
\hline 0.5 & 2 & 0.4549 & 7.2575 & 0.4778 \\
\hline 0.5 & 4 & 0.3507 & 6.4689 & 0.3952 \\
\hline 0.5 & 6 & 0.2921 & 5.7655 & 0.4011 \\
\hline 0.05 & 2 & 13.191 & 1.2828 & 0.5409 \\
\hline 0.05 & 4 & 10.968 & 1.9781 & 0.4935 \\
\hline 0.05 & 6 & 9.007 & 2.2315 & 0.4704 \\
\hline 0.02 & 2 & 71.1536 & 0.4904 & 0.6466 \\
\hline 0.02 & 4 & 63.4909 & 0.9991 & 0.6064 \\
\hline 0.02 & 6 & 53.9141 & 1.3018 & 0.5805 \\
\hline 0.01 & 2 & 253.433 & 0.3534 & 0.9822 \\
\hline 0.01 & 4 & 237.871 & 0.8563 & 0.9048 \\
\hline 0.01 & 6 & 207.192 & 1.0771 & 0.8847 \\
\hline
\end{tabular}

Table 9.6: Values of $t_{b t D}^{0}, \alpha$ for breakthrough time and $m$ for $q_{o D}$ for simultaneous variations of $q_{D}$ with $n_{o} . x_{e D}=20$ and $\mathrm{M}, h_{w D}$, and $n_{o}$ are equal to 1 , their base values.

developing correlations. Since more than one function could normally match the points, the criteria used to select the best function for each match was a maximum error of $5 \%$ in the simplest possible function. All the functions can be categorized in two main groups:

1. Polynomial functions:

$$
f(y)=A+B f(x)+C f(x)^{2}+D f(x)^{3}
$$

with $f(x)=x$ or $\ln (x)$ and $f(y)=y$ or $\ln (y)$.

2. Extensions of the linear functions:

$$
f(y)=A(B f(x)+C)^{D}+E
$$

with $f(x)=x^{a}$ or $\ln (x+a)$ or $e^{a x^{b}}$ and $\mathrm{f}(\mathrm{y})=\mathrm{y}$.

The parameters $t_{b t d}^{0}$ and $\alpha$ can be written as:

$$
t_{b t D}^{0}=f t\left(q_{D}\right) f t(M) f t\left(x_{e D}\right) f t\left(h_{w D}\right) f t\left(n_{o}\right) f t\left(n_{w}\right) f t\left(q_{D}, M\right) f t\left(q_{D}, x_{e D}\right) f t\left(q_{D}, n_{o}\right)
$$

and

$$
\alpha=f a\left(q_{D}\right) f a\left(h_{w D}\right) f a\left(n_{o}\right) f a\left(n_{w}\right) f a\left(q_{D}, M\right)
$$


where the functions $\mathrm{ft}$ and fa are given by either Equation 9.32 or Equation 9.33. The equations and functions to be used for each factor are given in Table 9.7. The values of each constant are given in Table 9.8 .

For example, to calculate $f t\left(q_{D}\right)$ in Equation 9.34 for $q_{D} \geq 0.1$, Table 9.7 gives the equation to be used as:

$$
f t\left(q_{D}\right)=A\left(B q_{D}{ }^{a}+C\right)^{D}+E
$$

with the constants given by the values shown in the first line of Table 9.8 .

The extra correction factors are given by:

$$
\begin{gathered}
f t\left(q_{D}, M\right)=\operatorname{aft}(M)+b f t(M) \ln \left(q_{D}\right) \\
f t\left(q_{D}, x_{e D}\right)=\operatorname{aft}\left(q_{D}\right)+b f t\left(q_{D}\right) \ln \left(x_{e D}\right) \\
f t\left(q_{D}, n_{o}\right)=\operatorname{aft}\left(n_{o}\right)+b f t\left(n_{o}\right) \ln \left(q_{D}\right) \\
f a\left(q_{D}, M\right)=\operatorname{afa}(M)+b f a(M) q_{D}
\end{gathered}
$$

where the factors are also shown in Tables 9.7 and 9.8 .

\subsubsection{Post-Breakthrough Behavior}

The correlations for post-breakthrough time behavior were derived using a solution based on vertical equilibrium, that can be written as [12]:

$$
q_{o D}=q_{D}\left(\frac{t_{b t D}}{2 t_{D}-t_{b t D}}\right)^{m}
$$

where $m$ is the parameter to be calculated by a nonlinear regression with the dimensionless variables and is equal to 0.5 for the vertical equilibrium assumption used by Tiefenthal [12].

The water-cut $f_{w}$ is given by:

$$
f_{w}=1-\frac{q_{o D}}{q_{D}}
$$

and WOR at standard conditions by:

$$
W O R=\frac{B_{o}}{B_{w}}\left(\frac{q_{D}}{q_{o D}}-1\right)
$$

The simulations were performed normally up to $t_{D}=3 t_{b t D}$ and for this range $m$ showed only small variations with the gridblock sizes, so that the results of the most refined grid of the pattern, that is, $\Delta z_{D}=0.005$, were used for each value of dimensionless variable to perform the regressions. The Gauss-Newton method [15] was used for these computations. These values are shown in Tables 9.4 and 9.5. When the dimensionless variables were not far from the base case, the runs demonstrated that the values of $m$ calculated by these matches can normally be used for $t_{D}$ of up to $50 t_{b t D}$. 


\begin{tabular}{|c|c|c|c|}
\hline Function & Equation & $f(x)$ & $\mathrm{f}(\mathrm{y})$ \\
\hline$f t\left(q_{D}\right), q_{D} \geq 0.1$ & 9.33 & $x^{a}$ & $y$ \\
\hline$f t\left(q_{D}\right), q_{D}<0.1$ & 9.32 & $\ln x$ & $\ln y$ \\
\hline$f t(M)$ & 9.32 & $\ln x$ & $\ln y$ \\
\hline$f t\left(x_{e D}\right)$ & 9.33 & $\ln (x+a)$ & $y$ \\
\hline$f t\left(h_{w D}\right)$ & 9.32 & $x$ & $y$ \\
\hline$f t\left(n_{o}\right)$ & 9.33 & $e^{a x^{b}}$ & $y$ \\
\hline$f t\left(n_{w}\right)$ & 9.33 & $e^{a x^{b}}$ & $y$ \\
\hline$a f t(M)$ & 9.32 & $\ln x$ & $\ln y$ \\
\hline$b f t(M)$ & 9.32 & $\ln x$ & $y$ \\
\hline$a f t\left(q_{D}\right)$ & 9.32 & $\ln x$ & $y$ \\
\hline$b f t\left(q_{D}\right)$ & 9.32 & $\ln x$ & $y$ \\
\hline $\operatorname{aft}\left(n_{o}\right)$ & 9.32 & $\ln x$ & $y$ \\
\hline$b f t\left(n_{o}\right)$ & 9.32 & $\ln x$ & $y$ \\
\hline$f a\left(q_{D}\right)$ & 9.32 & $\ln x$ & $\ln y$ \\
\hline$f a\left(h_{w D}\right)$ & 9.33 & $x^{a}$ & $y$ \\
\hline$f a\left(n_{o}\right)$ & 9.33 & $x^{a}$ & $y$ \\
\hline$f a\left(n_{w}\right)$ & 9.33 & $x^{a}$ & $y$ \\
\hline$a f a(M)$ & 9.33 & $x^{a}$ & $y$ \\
\hline$b f a(M)$ & 9.32 & $\ln x$ & $y$ \\
\hline$f m\left(q_{D}\right)$ & 9.32 & $\ln x$ & $\ln y$ \\
\hline$f m(M)$ & 9.32 & $\ln x$ & $y$ \\
\hline$f m\left(x_{e D}\right)$ & 9.32 & $\ln x$ & $\ln y$ \\
\hline$f m\left(h_{w D}\right)$ & 9.32 & $x$ & $y$ \\
\hline$f m\left(n_{o}\right)$ & 9.33 & $e^{a x^{b}}$ & $\mathrm{y}$ \\
\hline afm $\left(q_{D}\right)$ & 9.32 & $\ln x$ & $\ln y$ \\
\hline$b f m\left(q_{D}\right)$ & 9.32 & $\ln x$ & $y$ \\
\hline
\end{tabular}

Table 9.7: Equations and functions for the correlations of $t_{b t D}^{0}, \alpha$ and $m\left(q_{o D}\right)$. 


\begin{tabular}{|c|c|c|c|c|c|c|c|}
\hline Function & $\mathrm{a}$ & $\mathrm{b}$ & $\mathrm{A}$ & $\mathrm{B}$ & $\mathrm{C}$ & $\mathrm{D}$ & $\mathrm{E}$ \\
\hline$f t\left(q_{D}\right), q_{D} \geq 0.1$ & -0.36187 & - & 0.63589 & 0.32543 & 0.55970 & 7.20687 & -0.02716 \\
\hline$f t\left(q_{D}\right), q_{D}<0.1$ & - & - & 0.56278 & 1.01046 & 0.77215 & 0.06918 & - \\
\hline$f t(M)$ & - & - & 0.00237 & -0.29231 & 0.06269 & -0.00440 & - \\
\hline$f t\left(x_{e}\right)$ & 0.00023 & - & 1.0 & 0.51495 & -0.45280 & 0.05202 & 0.0 \\
\hline$f t\left(h_{w D}\right)$ & - & - & 0.12106 & -1.07741 & 4.23812 & -2.28177 & - \\
\hline$f t\left(n_{o}\right)$ & -0.07799 & 1.12590 & 1.0 & 1.09189 & 0.0 & 1.0 & 0.0 \\
\hline$f t\left(n_{w}\right)$ & 0.00833 & 1.0 & 1.0 & 0.99303 & 0.0 & 1.0 & 0.0 \\
\hline$a f t(M)$ & - & - & -0.02015 & -0.37233 & -0.05630 & 0.01269 & - \\
\hline$b f t(M)$ & - & - & -0.00173 & -0.13537 & -0.00481 & 0.00469 & - \\
\hline$a f t\left(q_{D}\right)$ & - & - & 0.96112 & -0.40901 & -0.20875 & -0.01565 & - \\
\hline$b f t\left(q_{D}\right)$ & - & - & 0.03092 & 0.18297 & 0.09483 & 0.00875 & - \\
\hline$a f t\left(n_{o}\right)$ & - & - & 1.0 & 0.14401 & -0.39431 & 0.11374 & - \\
\hline$b f t\left(n_{o}\right)$ & - & - & 0.0 & 0.05645 & -0.16935 & 0.05053 & - \\
\hline$f a\left(q_{D}\right)$ & - & - & 2.44506 & 0.74312 & -0.08108 & -0.01379 & - \\
\hline$f a\left(h_{w D}\right)$ & -5.16519 & - & 0.95795 & 0.92981 & -0.00023 & 0.34618 & 0.05673 \\
\hline$f a\left(n_{o}\right)$ & 0.43174 & - & 1.0 & 1.00177 & 0.0 & 1.0 & 0.0 \\
\hline$f a\left(n_{w}\right)$ & 1.0 & - & 1.0 & 0.33433 & 0.66566 & -2.73073 & 0.0 \\
\hline$a f a(M)$ & -0.16534 & - & 1.0 & 0.96120 & 0.0 & 1.0 & 0.0 \\
\hline$b f a(M)$ & - & - & 0.18891 & 1.61377 & 0.0 & 0.0 & - \\
\hline$f m\left(q_{D}\right)$ & - & - & -0.85501 & -0.22928 & -0.11837 & -0.02331 & - \\
\hline$f m(M)$ & - & - & 0.99762 & 0.09478 & -0.00288 & -0.00150 & 0.0 \\
\hline$f m\left(x_{e D}\right)$ & - & - & 1.05994 & -0.22414 & -0.14543 & 0.03404 & 0.0 \\
\hline$f m\left(h_{w D}\right)$ & - & - & 0.22840 & 0.7787 & 0.0 & 0.0 & 0.0 \\
\hline$f m\left(n_{o}\right)$ & -0.04130 & 1.0 & 1.0 & 1.03490 & 0.0 & 1.0 & 0.0 \\
\hline m & - & - & -0.35914 & 0.21539 & 0.17615 & 0.00654 & - \\
\hline$a f m\left(q_{D}\right), q_{D} \geq 0.02$ & - & - & 0.18917 & 0.13644 & 0.09088 & 0.02874 & - \\
\hline$b f m\left(q_{D}\right), q_{D} \geq 0.02$ & - & - & -0.07212 & 0.81646 & 0.50476 & 0.05771 & - \\
\hline$a f m\left(q_{D}\right), q_{D}<0.02$ & - & - & -0.20607 & -0.67350 & -0.33860 & -0.03607 & - \\
\hline$b f m\left(q_{D}\right), q_{D}<0.02$ & - & & & & & & \\
\hline
\end{tabular}

Table 9.8: Values of the constants for the correlations of $t_{b t D}^{0}, \alpha$ and $m\left(q_{o D}\right)$. A - means that the constant does not appear in the equation for that factor. 
The value $m=0.5$ can be used for a quick estimate of post-breakthrough behavior when the dimensionless variables are close to the base case, but as the dimensionless variables move away from the base case, $m$ varies considerably.

A procedure similar to that used to obtain the correlations for the parameters of breakthrough time was used for the parameter m. Again the functions described by Equations 9.32 and 9.33 can be applied. Dimensionless rates are still matched using their original values and other parameters are added as correction factors. An extra correction factor to account for simultaneous variations of $q_{D}$ and $x_{e D}$ was also added.

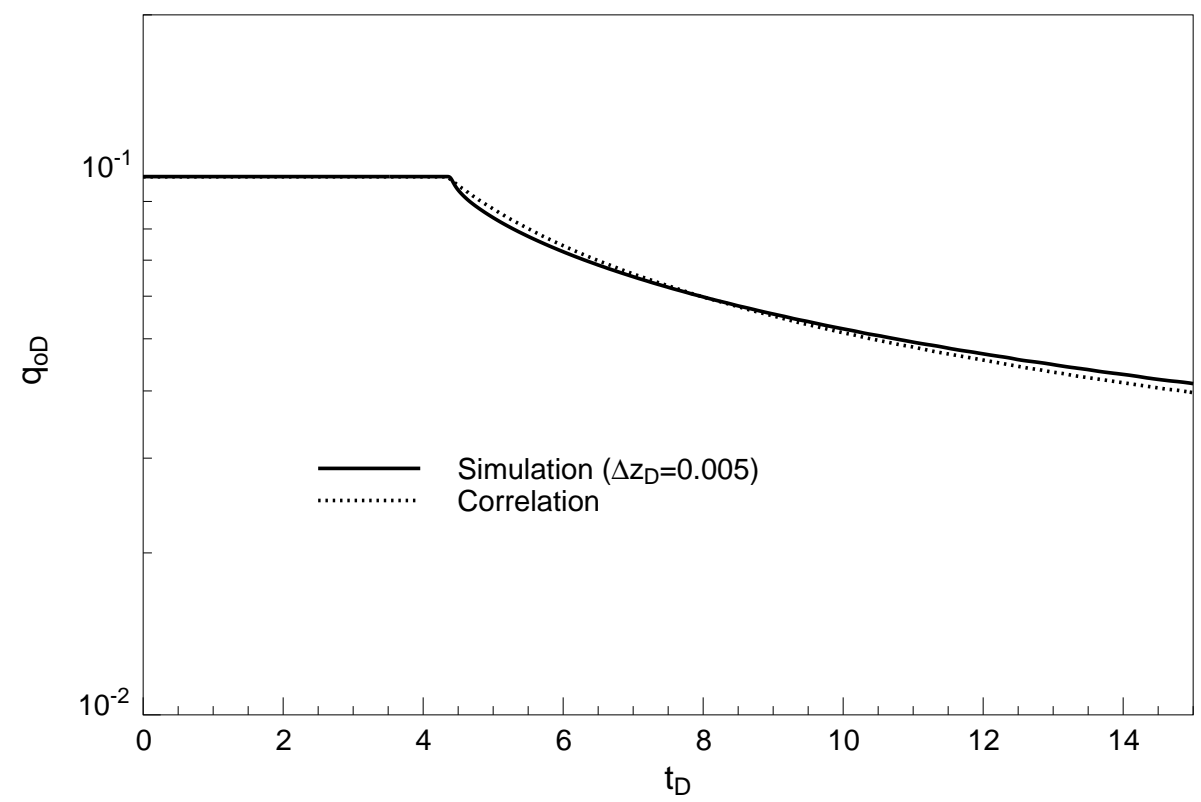

Figure 9.5: Comparison of breakthrough time and $q_{o D}$ correlations with simulation results for the base case.

The correlation can be written as:

$$
m=f m\left(q_{D}\right) f m(M) f m\left(x_{e D}\right) f m\left(h_{w D}\right) f m\left(n_{o}\right) f m\left(q_{D}, x_{e D}\right)
$$

where the functions and corrections factors are given by either Equation 9.32 or Equation 9.33 .

The correction factor $f m\left(q_{D}, x_{e D}\right)$ is given by:

$$
f m\left(q_{D}, x_{e D}\right)=a f m\left(q_{D}\right)+b f m\left(q_{D}\right) \ln \left(x_{e D}\right)
$$

The equations and functions to be used for each factor are given in Table 9.7. The values of each constant are given in Table 9.8.

Figure 9.5 shows a comparison of $q_{o D}$ vs $t_{D}$ calculated by the correlations and by simulations for the base case. The plot of WOR is in Figure 9.6. Except for a small deviation in the beginning of the water production, a good match is obtained.

\subsubsection{Cumulative Oil Recovery}

The dimensionless cumulative oil recovery is given by the area below the curve in Fig. 9.5. Numerically, it can be written for a given $t_{D}$ as: 


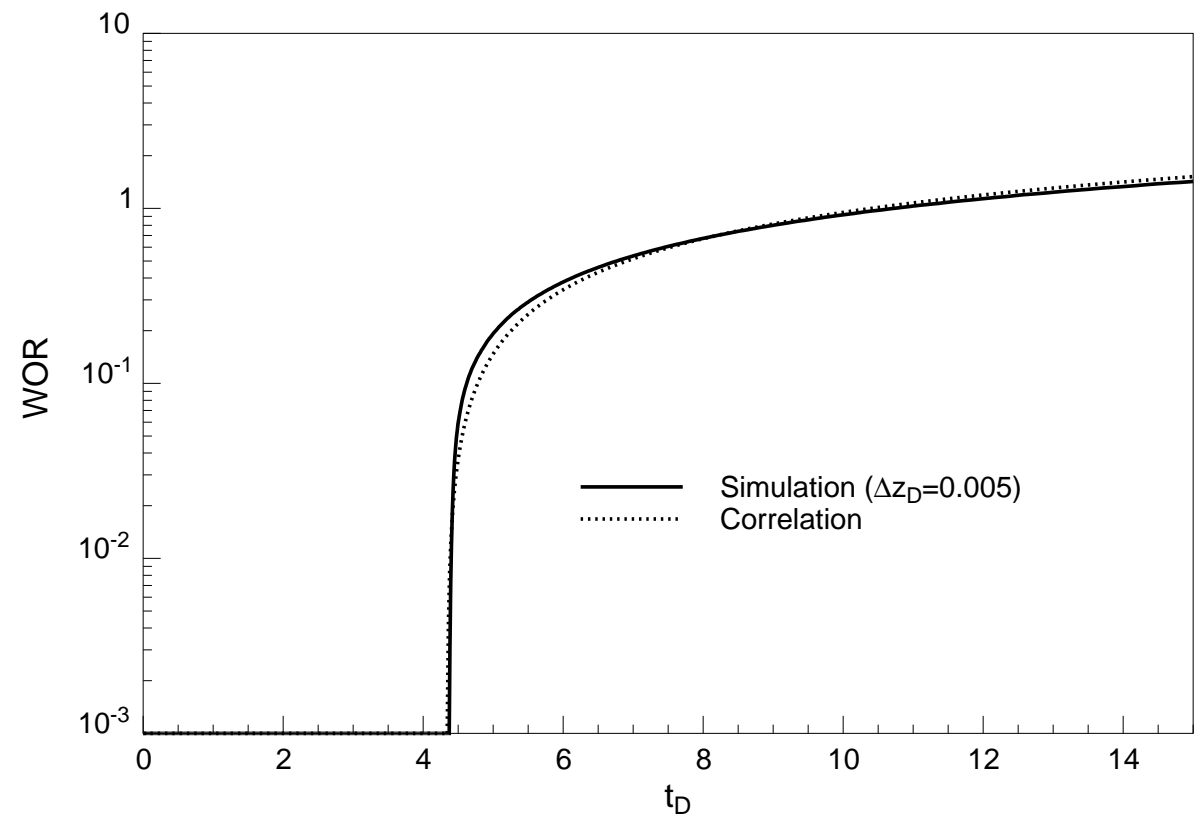

Figure 9.6: Comparison of breakthrough time and WOR correlations with simulation results for the base case.

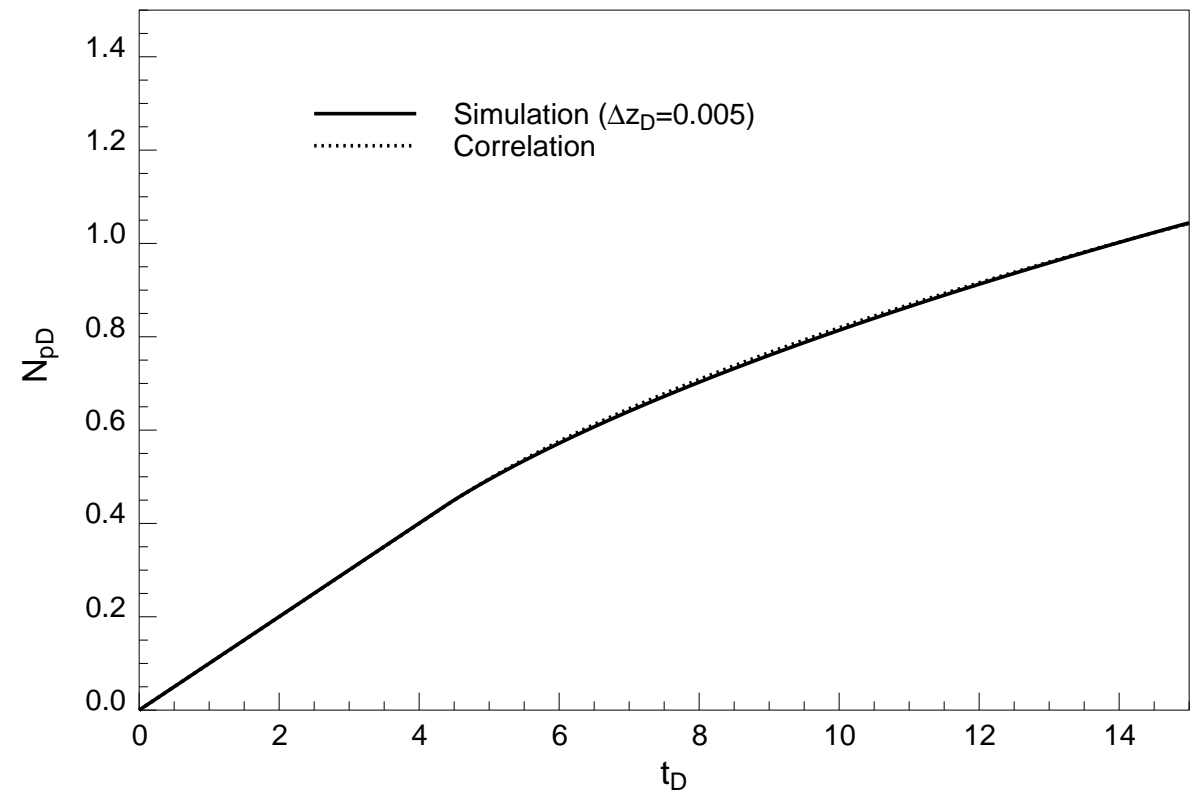

Figure 9.7: Comparison of $N_{p D}$ vs $t_{D}$ between the correlations and the simulation results for the base case. 


$$
N_{p D}=q_{D} t_{b t D}+\int_{t_{b t D}}^{t_{D}} q_{o D}(\tau) d \tau
$$

where the first term indicates $N_{p t b t D}$, that is, $N_{p D}$ up to breakthrough time and the second indicates $N_{p D}$ for post-breakthrough behavior. Using Equation 9.41 and integrating, $N_{p D}$ can be written as:

$$
N_{p D}=\frac{1-2 m}{2-2 m} N_{p t b t D}+\frac{1}{2-2 m}\left[q_{o D}\left(t_{D}\right)\left(2 t_{D}-t_{b t D}\right)\right]
$$

The plot of $N_{p D}$ vs $t_{D}$ for the base case for both simulation and correlation is shown in Figure 9.7, where a good match is still obtained.

\subsubsection{Optimum Grid}

The optimum grid is defined here as the gridblock size that gives the minimum number of blocks for the simulation with a small variation in breakthrough time, $N_{p D}$ and WOR behavior when compared with a refined grid. A refined grid is the gridblock size that gives practically no variation in those parameters when its dimension is reduced.

Here we just need to find the variation of the vertical block dimension $\Delta z_{D}$, since the horizontal dimension $\Delta x_{D}$ can be specified using the pattern described earlier.

Since $t_{b t D}^{0}$ is the limit for breakthrough time when the number of blocks in the vertical direction tends to infinity, this value can be used as a reference to calculate a refined or an optimum grid. Equation 9.31 can be used to find a value of $\Delta z_{D}$ for a specified ratio $t_{b t D}^{0} / t_{b t D}$.

At first, any practical criterion can be used to specify a refined vertical gridblock size. From what is reported in the literature and the experience with several runs, it is suggested that a vertical gridblock size that gives a difference less or equal to $1 \%$ in breakthrough time when compared with the parameter $t_{b t D}^{0}$ be used. From Equation 9.31, using $t_{b t D}=0.99 t_{b t D}^{0}$, a refined grid can be found as:

$$
\Delta z_{\text {Dref }}=0.01 \alpha^{-1}
$$

Similarly, it can be suggested that the optimum grid is the vertical gridblock size that obeys the following criteria:

1 - maximum variation of $5 \%$ in breakthrough time when compared with a refined vertical gridblock size result.

2 - Maximum variation of $3 \%$ in $N_{p D}$ at $t_{D}=3 t_{b t D}$ when compared with a refined vertical gridblock size result.

3 - Visual inspection for WOR variation, that normally loses its smooth behavior for coarse grids.

Using Equation 9.31, the criterion for breakthrough time (variation of $5 \%$ for a refined grid) can be written as:

$$
\Delta z_{\text {Dopt }}=0.06 \alpha^{-1}
$$

The runs showed that this criterion is normally enough to calculate the optimum grid, as the other two criteria defined above are also almost satisfied.

Table 9.9 shows the variation of breakthrough time and $N_{p D}$ for the base case for several $\Delta z_{D}$. Some of them are plotted in Fig. 9.8. Equation 9.48 gives $\Delta z_{\text {Dref }}=0.0062$ for 


\begin{tabular}{|c|c|c|c|c|}
\hline$\Delta z_{D}$ & $t_{b t D}$ & $\operatorname{Var}(\%)$ & $N_{p D}$ & $\operatorname{Var}(\%)$ \\
\hline 0.005 & 4.3547 & - & 0.9617 & - \\
\hline 0.01 & 4.3144 & 0.9 & 0.9555 & 0.6 \\
\hline 0.02 & 4.2542 & 2.3 & 0.9472 & 1.5 \\
\hline 0.03 & 4.2191 & 3.1 & 0.9419 & 2.1 \\
\hline 0.04 & 4.1111 & 5.5 & 0.9257 & 3.7 \\
\hline 0.05 & 4.0522 & 6.9 & 0.9162 & 4.7 \\
\hline 0.1 & 3.8794 & 8.6 & 0.8872 & 7.7 \\
\hline 0.2 & 3.5770 & 17.8 & 0.8292 & 13.8 \\
\hline
\end{tabular}

Table 9.9: Variations in breakthrough time and $N_{p D}$ with vertical gridblock size for the base case.

this situation, which can be roughly rounded to 0.005. Using Equation 9.49, the optimum grid for the base case is $\Delta z_{D}=0.038$, which can be rounded to 0.04 , the value used here.

\subsection{Applications}

Besides the immediate calculation of breakthrough time, $q_{o D}, f_{w}$, WOR, $N_{p}, \mathrm{RF}$ and optimum grid, the derived correlations can be used for some additional applications.

\subsubsection{Maximum Rate}

The derived correlations allow the calculation of any dimensionless variable if the others and the breakthrough time are known. Then, for a given $t_{b t D}^{0}$, a maximum rate to be imposed on the production well can be found. This means that any rate below this $q_{\text {max }}$ gives a breakthrough time longer than the desired one. This maximum rate is not the critical rate in the classical meaning, since the boundary conditions used to develop these correlations are no-flow boundaries. Some works in the literature, however, refer to this rate as the critical rate [16] [17].

The value of $q_{\text {Dmax }}$ can not be calculated explicitly from Equation 9.34, so a numerical procedure must be performed to obtain it. Newton's method was chosen here for such numerical calculations. The equation to be solved is:

$$
F\left(q_{D}\right)=\operatorname{Aft}\left(q_{D}\right) f t\left(q_{D}, M\right) f t\left(q_{D}, x_{e D}\right) f t\left(q_{D}, n_{o}\right)-t_{b t D}^{0}=0
$$

where $\mathrm{A}$ is the product of the other factors in Equation 9.34. Once $q_{D \max }$ in known, $q_{\max }$ can be calculated from Equation 9.12.

\subsection{A field produced by several wells}

For a field with evenly spaced wells as shown in Figure 9.9 and equal production rate for each well, the total production can be calculated using the derived correlations for any number of wells, located at the same reservoir height. 


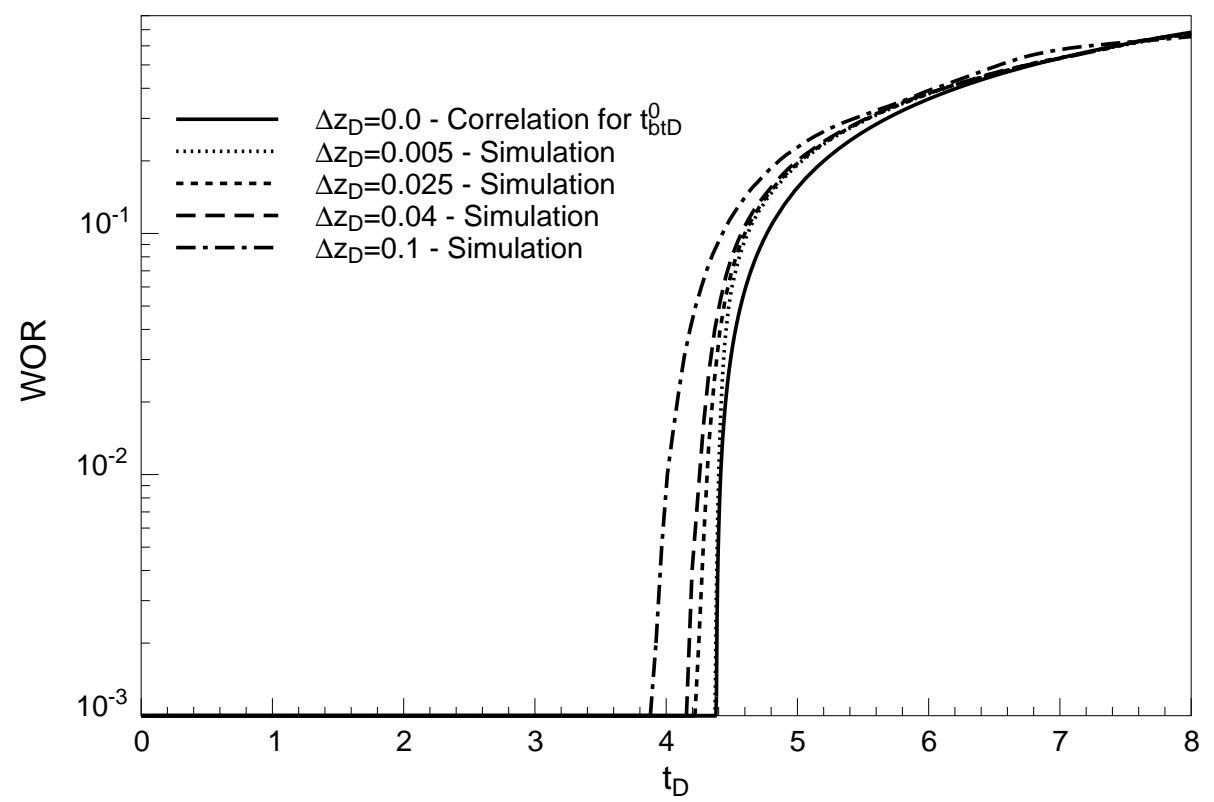

Figure 9.8: Effect of vertical gridblock size on WOR as a function of time.

The rate of each well is given by:

$$
q=\frac{q_{T}}{n_{w e l}}
$$

The dimensionless rate $q_{D}$ can be calculated by Equation 9.12. The drainage distance $x_{e D}$ for each well is given by:

$$
x_{e D}=\frac{X_{D}}{2 n_{w e l}}
$$

where $X_{D}$ is the dimensionless total extent of the reservoir, given by:

$$
X_{D}=\frac{X}{h_{o}} \sqrt{\left(\frac{k_{v}}{k_{h}}\right)}
$$

$N_{p D}$ for the entire field can be calculated by Equation 9.47 using the total dimensionless rate $q_{D T}$. Figure 9.10 shows the cumulative oil recovery for the entire field with four wells using the rate of the base case as the rate $q_{T}$. Again, the match with simulation when all wells are considered is good.

\subsection{Estimation of breakthrough time}

One relevant application of the procedure used to correlate $t_{b t D}$ with $\Delta z_{D}$ is the calculation of an accurate breakthrough time from coarse grid simulations. For the simulation of any field, the pattern described in Section 9.3 can be used to find the value of $t_{b t D}^{0}$ using just two coarse grid simulations. This is shown in Figure 9.11. The two points on the straight line represent the coarse grid simulations for $\Delta z_{D}=0.05$ and $\Delta z_{D}=0.04$. Matching these 


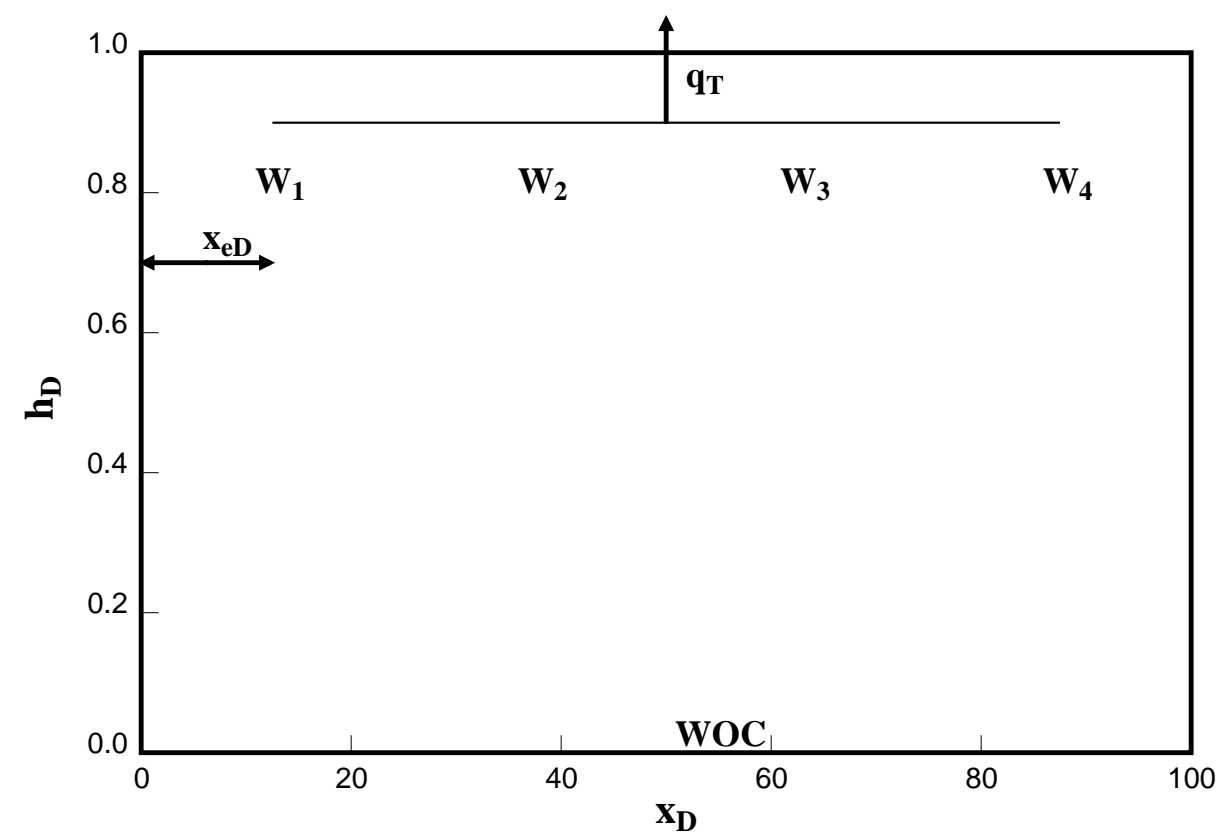

Figure 9.9: Evenly spaced wells in a field with a specified total rate

two points with a straight line, the extrapolation of the line to $\Delta z_{D}=0$ gives the value of $t_{b t D}^{0}$, which represents the breakthrough time for an infinite number of blocks in the vertical direction. This value of breakthrough time can then be used to predict the performance of the reservoir.

\subsection{Pseudofunctions}

The application described in the last section can be extended for the development of pseudofunctions to match the breakthrough time of coarse gridblock size simulations with breakthrough time from refined ones. The main idea is to change one of the dimensionless variables related with the shape of the relative permeability curves, that is, the exponents $n_{o}$ or $n_{w}$ or one of their end-points (which ultimately changes the mobility ratio $\mathrm{M}$ ), so that the velocity of the flow is also changed and this change compensates for the numerical diffusion created with the use of coarse gridblock sizes.

Section 9.4 described how the families of straight lines of $t_{b t D}$ vs $\Delta z_{D}$ were obtained by linear regression. Figure 9.12 shows straight lines from the family of $n_{o}$. The idea is to use a coarse grid simulation $\left(\Delta z_{D}=0.05\right.$ in Figure 9.12) and obtain the same breakthrough time if a refined grid simulation $\left(\Delta z_{D}=0.005\right.$ Figure 9.12) was used. In other words, the objective is to find a value of $n_{o p}$ (pseudo $n_{o}$ ) that matches the breakthrough time from a coarse grid simulation that uses $n_{o}$ and $\Delta z_{D}\left(\Delta z_{D}\left(n_{o}\right)\right)$, with that from a smaller $\Delta z_{D p}\left(\Delta z_{D}\left(n_{o p}\right)\right)$. The value $t_{b t D p}\left(t_{b t D}\left(n_{o p}\right)\right)$ for the refined $\Delta z_{D p}$ can be obtained throughout the straight line of $n_{o}$, that is, from the point A to point B shown in the Figure. The value of $n_{o p}$ is then obtained by extending a horizontal straight line from this value (point B) toward right to the vertical line that passes $\Delta z_{D}$ (point C). The value of $n_{o}$ from the family of straight lines that crosses this latter point is $n_{o p}$. Normally, an interpolation is needed for this graphical procedure. 


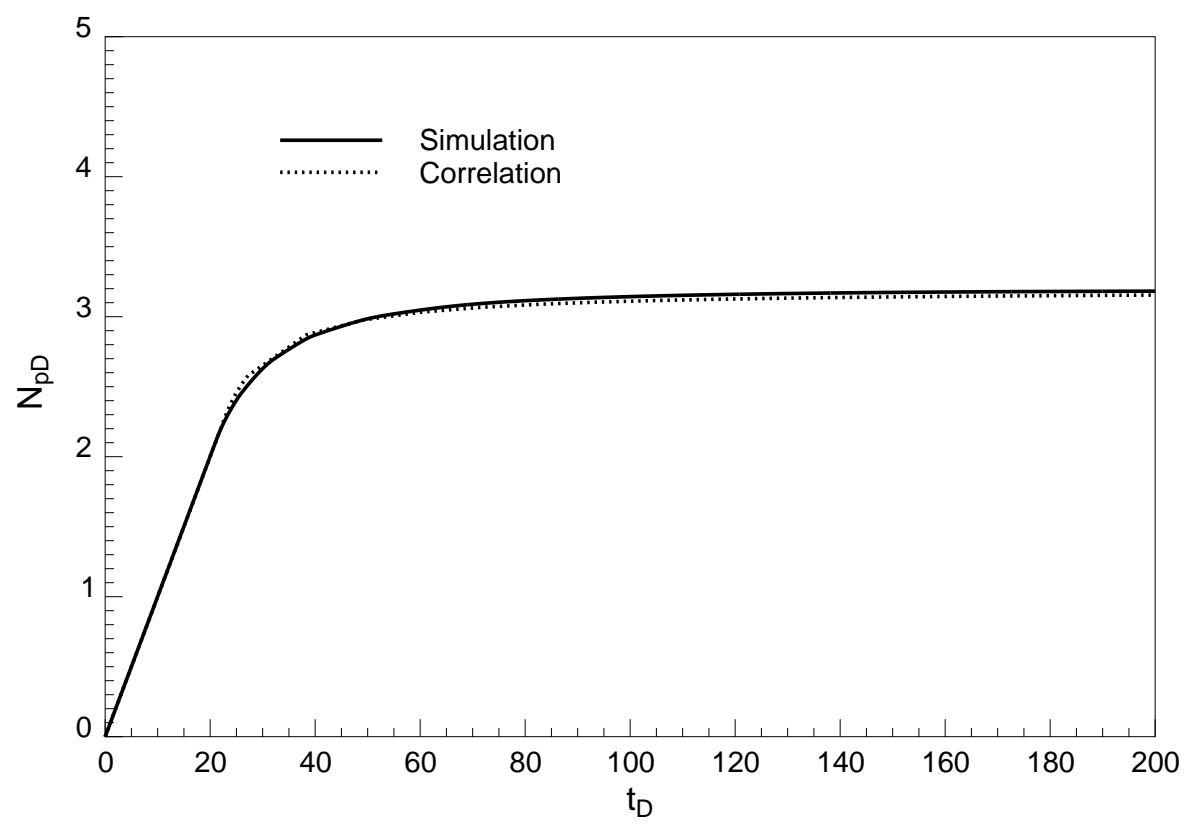

Figure 9.10: $N_{p D}$ vs $t_{D}$ curves for a field with four wells

Numerically, this means that we need to use Equation 9.31 to calculate $n_{o p}$, once $t_{b t D p}$ is known. As in the case of the calculation of maximum rate, a numerical procedure is needed to obtain $n_{o p}$. Again, Newton's method is applied. Using the values of $n_{o p}$ and $t_{b t D p}$ in Equation 9.31 and rearranging, the equation to be solved is:

$$
F\left(n_{o p}\right)=\ln \left[\operatorname{Aft}\left(n_{o p}\right) f t\left(q_{D}, n_{o p}\right)\right]-B f a\left(n_{o p}\right)=0
$$

Where $\mathrm{A}$ is the product of the other factors in Equation 9.34 divided by $t_{b t D p}$ and $\mathrm{B}$ is the product of the other factors in Equation 9.35 times $\Delta z_{D}\left(n_{o}\right)$.

Figure 9.13 shows an application for the base case for $n_{o}$ pseudofunction. The original value of $n_{o}$ is 2 and the procedure described above leads to a value of $n_{o p}$ equal to 1.08. The match of breakthrough time is practically exact, with some difference in WOR at the beginning but, after that, all curves merge into one.

It should be noted that the new value of $n_{o p}$ is always less than the original value $n_{o}$, which means that $n_{o p}$ can be less than 1 , a situation where the relative permeability curve does not have a physical meaning, but still can be used to match the breakthrough time. The procedure to obtain pseudofunctions based on $n_{w}\left(n_{w p}\right)$ and $\mathrm{M}\left(M_{p}\right)$ is similar to that for $n_{o p}$.

The procedures described above can be used in any simulation regardless of correlations. For this, two coarse grid simulations using the suggested pattern must be performed for each value of $n_{o}, n_{w}$ or $\mathrm{M}\left(k_{\text {rwro }}\right)$. These simulations generate a family of straight lines that can be used to calculate the new value $n_{o p}, n_{w p}$ or $M_{p}$ by a procedure similar to that described above. Fig. 9.14 shows an example of such a procedure. Each straight line corresponds to a value of a dimensionless variable $n_{o}, n_{w}$ or $M$. Using the graphical procedure described to obtain the pseudofunctions, the value of the dimensionless variable that matches breakthrough time can be found by interpolation from the straight lines. This value can be 


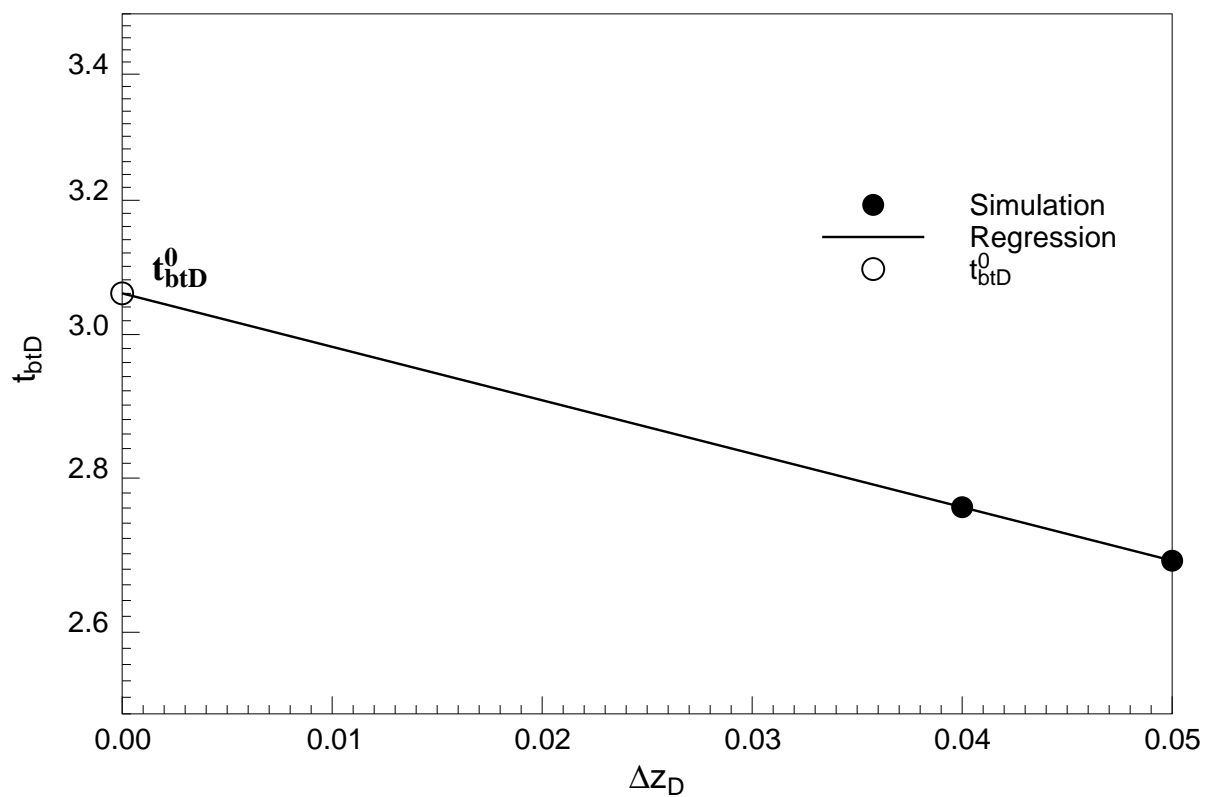

Figure 9.11: Extrapolation of two coarse grid simulations to find $t_{b t D}^{0}$.

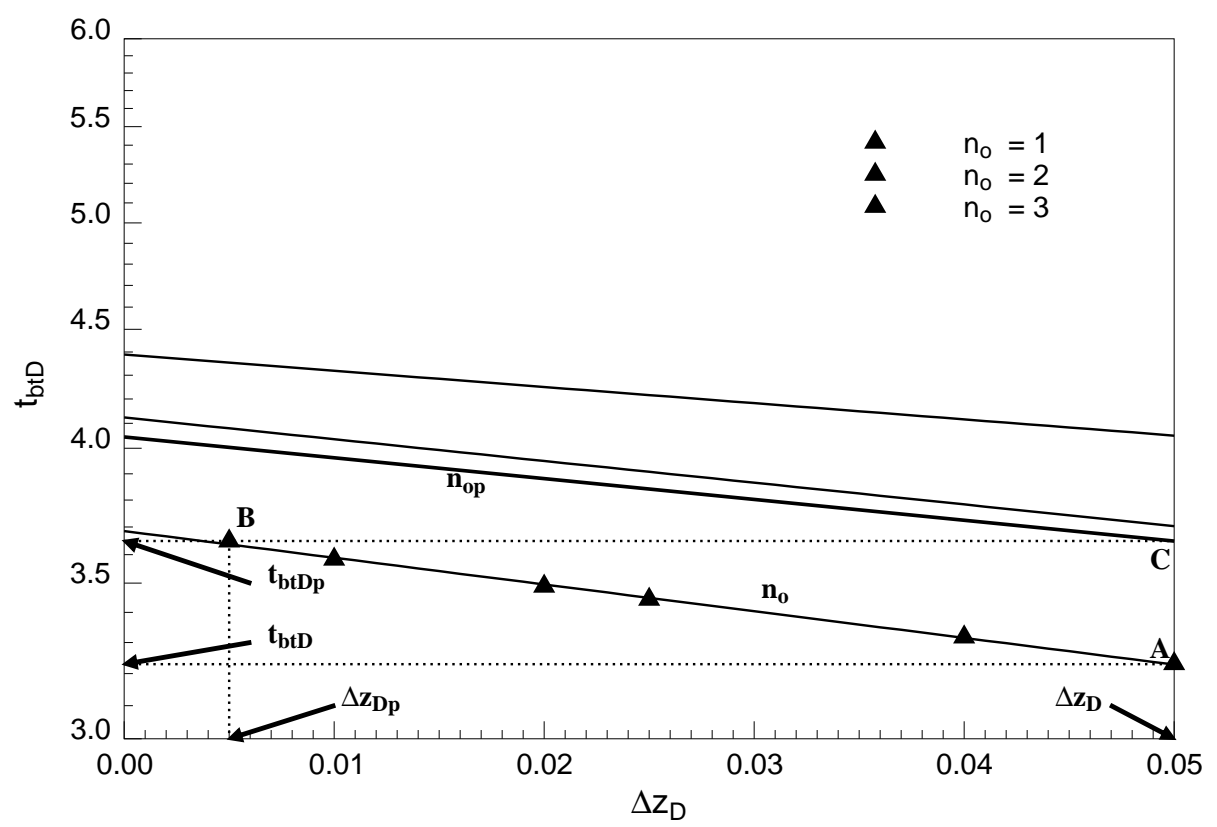

Figure 9.12: Procedure to obtain $n_{o p}$ for a given $n_{o}$ and $\Delta z_{D}$. 


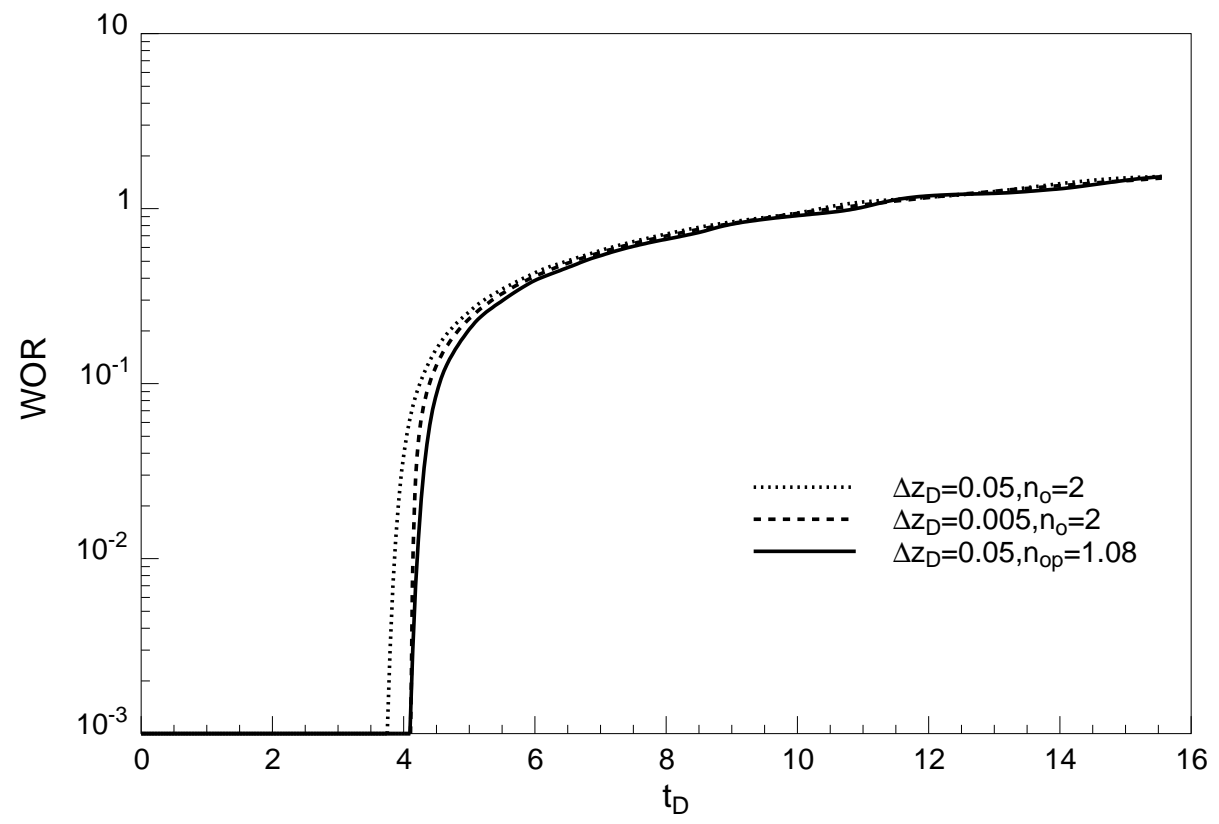

Figure 9.13: Application of pseudo $n_{o}$ for the base case data.

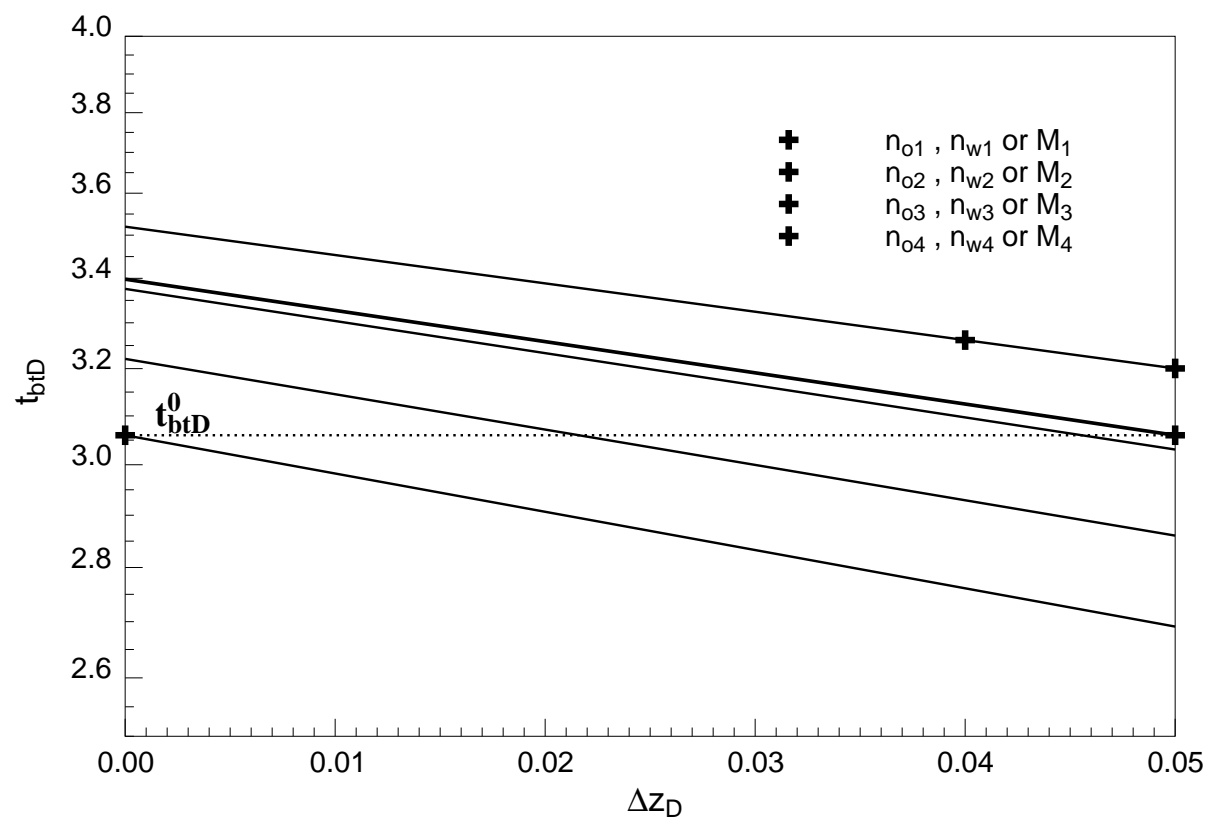

Figure 9.14: Variation of $n_{o}, n_{w}$ or $M$ to generate pseudofunctions for any simulation. 
used to match breakthrough time from one of the coarse grid simulations with the value of $t_{b t D}^{0}$ or a value of $t_{b t D}$ of a refined grid block.

\subsubsection{Gas Cresting Behavior}

The correlations were derived for water-oil flow, but since only dimensionless variables were used and the flow is governed mainly by the differences in densities and mobilities, they can be used exactly in the same way for gas-oil flow. The parameters and subscripts used for water should be now used for gas instead.

$$
G O R=\frac{B_{o}}{B_{g}}\left(\frac{q_{D}}{q_{o D}}-1\right)+R_{s}
$$

Figure 9.15 shows one example of breakthrough time and GOR performance for a gas-oil case application using the same reservoir parameters as the base case, but with $\Delta \rho=400 \mathrm{~kg} / \mathrm{m}^{3}, B_{g}=0.012$ and $\mathrm{M}=10$. The cumulative oil recovery is shown in Fig. 9.16. Both matches show that the correlations derived are robust and they can also be used for gas-oil flow with good accuracy.

\section{$9.9 \quad$ A Field Example}

A program was developed for the correlations and applications shown in this work and applied for a real field. The data for the field were obtained from an input data for the ECLIPSE simulator [14] supplied by the field operator and are shown in Table 9.10. The relative permeability data are given in Tables 9.11 and 9.12.

The field has both an aquifer and a gas cap and is produced by a horizontal well placed in the middle of the oil column. Since the correlations were derived for two-phase flow, they were applied for each phase separately assuming the well penetrated the entire width of the reservoir, made equal to the well length. For this situation, only half of the oil column must be used to apply the correlations. This means that the well will be at the top of the bottom half of reservoir for water-oil flow calculations and at the bottom of the top half of the reservoir for gas-oil flow calculations. It is assumed only half of the total rate to be used for each situation. This assumption is only valid until breakthrough time and if the flow resistances above and below the well are the same, that is, the contrast in densities and mobilities are the same for water-oil and gas-oil flows. This is not the case for this real field, but this assumption can be used as a first approach to check the accuracy of the correlations when applied using field data.

\subsubsection{Water-oil flow}

The water data generate the dimensionless variables shown in Table 9.13. Also shown in the same Table are the values of $t_{b t D}$ and $m$ calculated for these data using the derived correlations.

The results given by the correlations were compared with two simulations using the ECLIPSE simulator [14]: (1) the two-phase flow using the half of the oil column and half of the total rate with the well at the top of the reservoir and, (2) a three-phase flow using the original data for the field, with the well centered in the reservoir. 


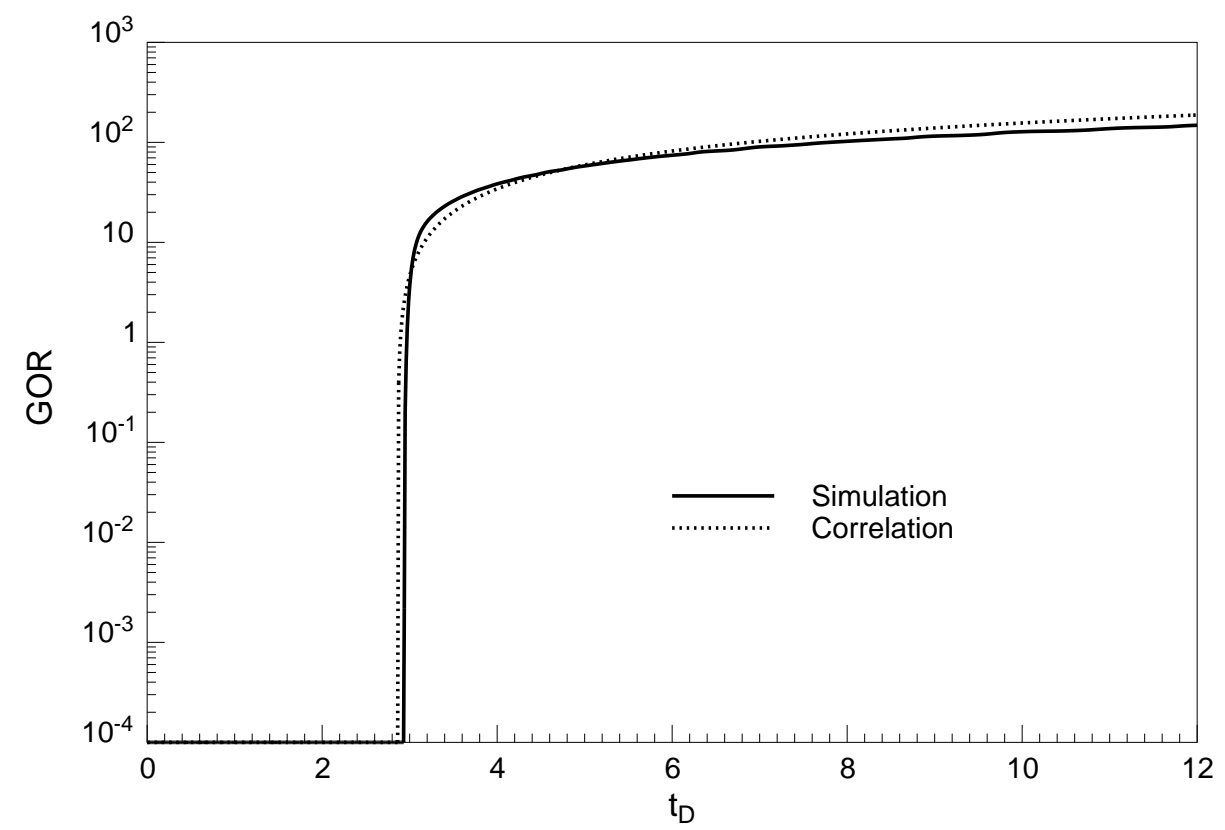

Figure 9.15: Comparison of breakthrough time and GOR from correlations with simulation results for gas-oil flow

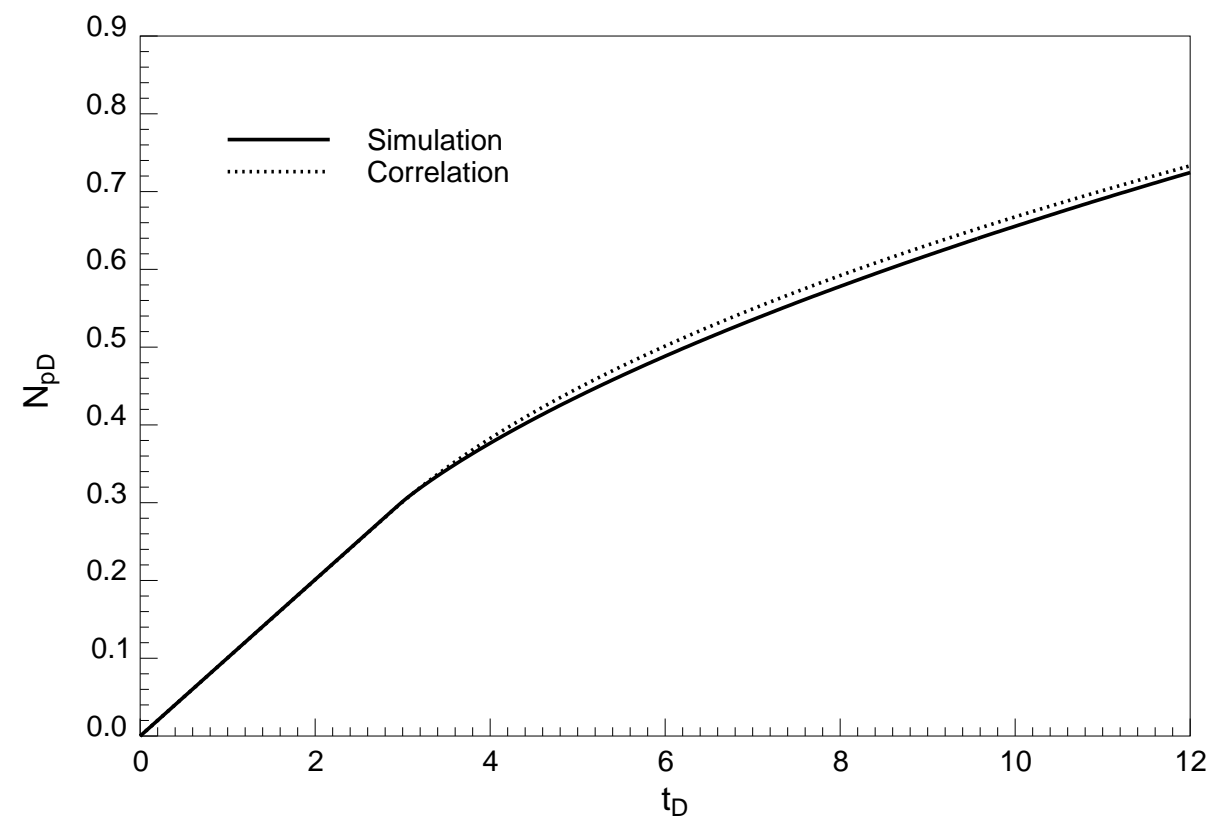

Figure 9.16: Comparison of $N_{p D}$ calculated from correlations to simulation results for gas-oil flow 


\begin{tabular}{|l|l|}
\hline Oil column, $h$ & $40 \mathrm{~m}$ \\
\hline Reservoir width,$W$ & $1300 \mathrm{~m}$ \\
\hline Reservoir extension, $X$ & $900 \mathrm{~m}$ \\
\hline Horizontal permeability, $k_{h}$ & $3 \mathrm{~d}$ \\
\hline Vertical permeability, $k_{v}$ & $1 \mathrm{~d}$ \\
\hline Porosity, $\phi$ & 0.2 \\
\hline Oil formation volume factor, $B_{o}$ & $1.965 \mathrm{rm}^{3} / \mathrm{sm}^{3}$ \\
\hline Water formation volume factor, $B_{w}$ & $1.03 \mathrm{rm}^{3} / \mathrm{sm}^{3}$ \\
\hline Gas formation volume factor, $B_{g}$ & $0.004 \mathrm{rm}^{3} / \mathrm{sm}^{3}$ \\
\hline Oil density, $\rho_{o}$ (standard) & $784.7 \mathrm{~kg} / \mathrm{m}^{3}$ \\
\hline Water density, $\rho_{w}$ (standard) & $1065 \mathrm{~kg} / \mathrm{m}^{3}$ \\
\hline Gas density, $\rho_{g}$ (standard) & $0.995 \mathrm{~kg} / \mathrm{m}^{3}$ \\
\hline Gas-oil solubility ratio, $R s$ & $312 \mathrm{sm} / \mathrm{sm}^{3}$ \\
\hline Oil viscosity, $\mu_{o}$ & $0.22 \mathrm{cp}$ \\
\hline Water viscosity, $\mu_{w}$ & $0.5 \mathrm{cp}$ \\
\hline Gas viscosity, $\mu_{o}$ & $0.02 \mathrm{cp}$ \\
\hline Well length, $L$ & $500 \mathrm{~m}$ \\
\hline Well height, $h_{w}$ & $20 \mathrm{~m}$ \\
\hline Production rate, $q$ & $1000.00 \mathrm{~m}^{3} / \mathrm{day}$ \\
\hline
\end{tabular}

Table 9.10: Field data set used to apply the developed correlations.

\begin{tabular}{|c|c|c|}
\hline$S_{w}$ & $k_{\text {rw }}$ & $k_{\text {row }}$ \\
\hline 0.260 & 0.0 & 0.890 \\
\hline 0.314 & 0.0116 & 0.623 \\
\hline 0.368 & 0.0232 & 0.427 \\
\hline 0.422 & 0.0348 & 0.276 \\
\hline 0.476 & 0.0580 & 0.169 \\
\hline 0.530 & 0.0928 & 0.107 \\
\hline 0.584 & 0.1421 & 0.0445 \\
\hline 0.638 & 0.2175 & 0.0267 \\
\hline 0.692 & 0.3103 & 0.0178 \\
\hline 0.746 & 0.4353 & 0.0045 \\
\hline 0.800 & 0.580 & 0.0 \\
\hline
\end{tabular}

Table 9.11: Water-oil relative permeability curves for field data. 


\begin{tabular}{|c|c|c|}
\hline$S_{g}$ & $k_{r g}$ & $k_{\text {rog }}$ \\
\hline 0.0 & 0.0 & 0.890 \\
\hline 0.074 & 0.0275 & 0.3218 \\
\hline 0.148 & 0.0688 & 0.209 \\
\hline 0.222 & 0.1100 & 0.1348 \\
\hline 0.296 & 0.1650 & 0.0825 \\
\hline 0.370 & 0.2283 & 0.04125 \\
\hline 0.444 & 0.2943 & 0.02475 \\
\hline 0.518 & 0.3768 & 0.0110 \\
\hline 0.592 & 0.4593 & 0.0055 \\
\hline 0.740 & 0.5500 & 0.0 \\
\hline
\end{tabular}

Table 9.12: Gas-oil relative permeability curves for field data.

\begin{tabular}{|c|c|}
\hline$q_{D}$ & 0.0052 \\
\hline $\mathrm{M}$ & 0.287 \\
\hline$x_{e D}$ & 13.0 \\
\hline$h_{w D}$ & 1.0 \\
\hline$n_{o}$ & 3.25 \\
\hline$n_{w}$ & 2.70 \\
\hline$t_{b t D}$ & 477.0 \\
\hline$m$ & 1.84 \\
\hline
\end{tabular}

Table 9.13: Dimensionless variables for water-oil flow for the field data. 


\begin{tabular}{|c|c|}
\hline$q_{D}$ & 0.0085 \\
\hline $\mathrm{M}$ & 6.798 \\
\hline$x_{e D}$ & 13.0 \\
\hline$h_{w D}$ & 1.0 \\
\hline$n_{o}$ & 7.35 \\
\hline$n_{w}$ & 1.23 \\
\hline$t_{b t D}$ & 211.0 \\
\hline$m$ & 1.58 \\
\hline
\end{tabular}

Table 9.14: Dimensionless variables for gas-oil flow for real field data.

Figure 9.17 shows the comparison between these runs with the results of the correlations for $W O R$ vs $t($ days $)$. The value of breakthrough time calculated from the correlations is $t=618$ days, which compared with the value $t=638$ days calculated by the simulator for two-phase flow gives a difference of $3 \%$, which is with in the expected range of errors of the correlations. The WOR behavior also shows that the correlations give good results for the field situation.

The results of the simulation for three-phase flow shows larger deviations from the correlations. The difference in breakthrough time, although still small (3\%), is caused by the differences in the properties of water and gas. As explained, the use of half of the rate for each two-phase flow is only valid if the contrasts in densities and mobilities are the same for water-oil and gas-oil flow. The larger differences in WOR are due to another factor besides the contrast in densities and mobilities between water and gas: the well calculations performed by the simulator after breakthrough time are made using three-phase relative permeabilities [14], which changes both WOR and GOR behaviors. However, the general behavior of simulation and correlation results is very similar.

\subsubsection{Gas-oil flow}

The gas data generate the dimensionless variables shown in Table 9.14. Also shown in the same table are the values of $t_{b t D}$ and $m$ calculated for these data using the derived correlations.

The results of the correlations were again compared with those from the ECLIPSE simulator using two approaches: (1) two-phase flow using the half of the oil column with the well at the bottom of the reservoir and, (2) three-phase flow using the original data for the field, with the well centered in the reservoir, that is, the same simulation used to obtain the results for water-oil flow discussed in Section 9.9.1.

Figure 9.18 shows the comparison between these runs and the results of the correlations for $G O R$ vs $t($ days $)$. The value of $t_{b t}$ calculated by the correlations is $t=580$ days, which compared with the value $t=565$ days from two-phase simulation gives again a difference of about $3 \%$. The GOR behavior shows larger differences, which can be expected since the dimensionless variables have extreme values in this case, far from the base case studied. However, the correlations still gives a good estimate of the post-breakthrough behavior.

The results of the three-phase simulation when compared with those obtained from 


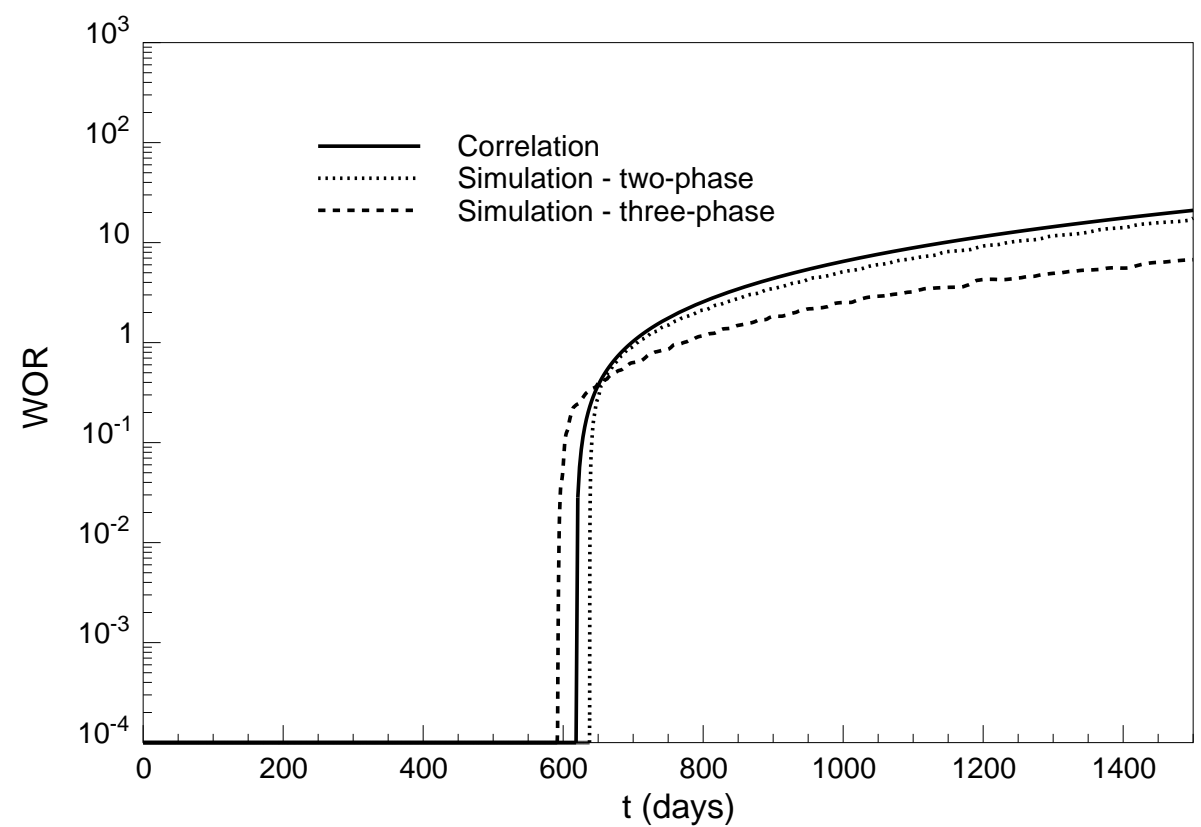

Figure 9.17: Comparison of breakthrough time and WOR correlations with simulation results for a field application.

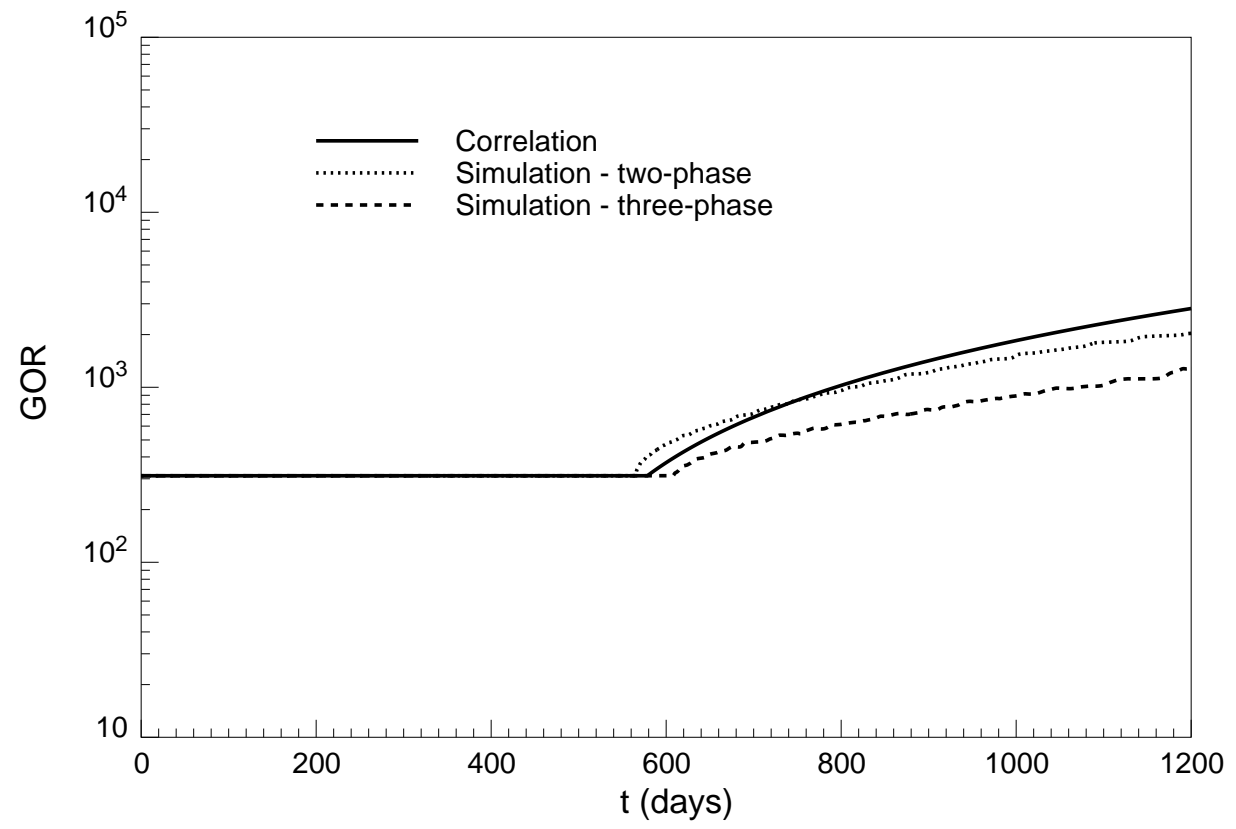

Figure 9.18: Comparison of breakthrough time and GOR correlations with simulation results for a field application. 
the correlations again show larger differences. As in the water-oil case, these differences are due to the differences in properties of the water and gas and the three-phase relative permeability calculations performed by the simulator after breakthrough time. However, the general behavior is not far from that found by the correlations.

\subsection{Conclusions}

Water and gas cresting behavior are important reservoir phenomena that occur in reservoirs with aquifers and/or gas caps. A good understanding of this phenomenon along with calculations based on simple correlations forms a good basis to start a reservoir simulation study. The correlations and procedures developed in this work help to improve this understanding.

The conclusions of this work can be summarized as follows:

1. A set of dimensionless variables has been defined, leading to clear identification of important parameters for a reservoir study.

2. A grid pattern to start a reservoir simulation study has been suggested. The proposed pattern leads to minor loss of accuracy in the results compared with very refined grid results and is useful to generate procedures to calculate breakthrough time, develop correlations and generate pseudofunctions.

3. A new set of correlations for breakthrough time, post breakthrough behavior and optimum grid has been developed using just dimensionless variables derived from the flow equations. These correlations take into account the main reservoir parameters that affect the flow, including the effects of relative permeability end-points and shapes. Numerical diffusion effects are taken into account by the inclusion of grid block sizes in the correlations. The correlations can be used for either water-oil or gas-oil flow. Such correlations can be used at the beginning of a reservoir study to estimate breakthrough time, post-breakthrough behavior and give an initial estimate of for the grid block sizes to use in the simulation runs.

4. A procedure to calculate an accurate breakthrough time using just coarse grid simulations and the suggested pattern has been presented.

5. Pseudofunctions to match breakthrough time using coarse grid runs have been generated. These pseudofunctions can be used together with the developed correlations or even independently, to obtain the right breakthrough time even with a coarse grid.

6. Applicability of the correlations to an entire field with multiple wells has been demonstrated.

7. A real field example has shown that the correlations are reliable and can be used at the beginning of a reservoir simulation study to give an estimate of breakthrough time and post-breakthrough behavior. 


\section{Nomenclature}

\begin{tabular}{|c|c|}
\hline$B$ & formation volume factor, $\mathrm{m}^{3} / \mathrm{m}^{3}$ \\
\hline$c$ & compressibility, $P a^{-1}$ \\
\hline$D$ & depth, distance from the surface, $m$ \\
\hline$f_{w}$ & fractional water flow (water-cut) \\
\hline$F V F$ & formation volume factor, $\mathrm{m}^{3} / \mathrm{m}^{3}$ \\
\hline$g$ & gravitational acceleration $=9.81 \mathrm{~m} / \mathrm{s}^{2}$ \\
\hline GOC & gas-oil contact \\
\hline GOR & gas-oil ratio \\
\hline$h$ & height \\
\hline$h_{w}$ & well height \\
\hline$k$ & permeability, $m^{2}$ \\
\hline$l$ & phase \\
\hline$L$ & well length, $m$ \\
\hline$M$ & mobility ratio \\
\hline$m$ & exponent for $q_{0 D}$ \\
\hline$N$ & cumulative oil recovery, $m$ \\
\hline$n_{l}$ & exponent for $k r_{l}$ \\
\hline$n_{b}$ & number of blocks \\
\hline$p$ & pressure, $P a$ \\
\hline$q$ & rate at reservoir conditions, $\mathrm{m}^{3} / \mathrm{s}$ \\
\hline$Q$ & rate at standard conditions, $\mathrm{m}^{3} / \mathrm{s}$ \\
\hline$R s$ & solubility ratio, $m^{3} / m^{3}$ \\
\hline$R F$ & Recovery Factor \\
\hline$r$ & radial direction, $m$ \\
\hline$s$ & length in the streamline direction, $m$ \\
\hline$S$ & saturation \\
\hline$t$ & time, $s$ \\
\hline$u$ & velocity, $m / s^{2}$ \\
\hline$V$ & volume,$m^{3}$ \\
\hline$x$ & main horizontal direction, with the origin in the well, $m$ \\
\hline$W$ & width (y direction), $m^{3}$ \\
\hline$W O C$ & water-oil contact \\
\hline WOR & water-oil ratio \\
\hline$y$ & horizontal y direction, $m$ \\
\hline$z$ & vertical direction \\
\hline
\end{tabular}

\section{Greek}

$\alpha \quad$ slope of the straight lines for $t_{b t D}$

$\gamma \quad$ hydrostatic gradient $=\rho g, P a / m$

$\lambda \quad$ phase mobility Pa.s $\mathrm{s}^{-1}$

$\mu \quad$ viscosity $P$ a.s

$\rho \quad$ density, $\mathrm{kg} / \mathrm{m}^{3}$ 


$\begin{array}{ll}\phi & \text { porosity } \\ \psi & \text { ratio between the density differences } \\ \Phi & \text { potential, } \mathrm{Pa} \\ \theta & \text { angle } \\ \zeta & \text { mass fraction of each component }\end{array}$

\section{Subscripts}

\begin{tabular}{ll} 
b & block \\
bp & bubble point \\
bt & breakthrough \\
c & critical \\
cp & component \\
D & dimensionless \\
e & external ( lateral edge ) \\
ef & effective \\
g & gas \\
h & horizontal direction \\
i & grid block discretization index \\
l & phase \\
o & oil \\
opt & optimum \\
or & oil residual \\
p & pseudo function \\
r & relative \\
rc & rock \\
ref & refined \\
res & reservoir \\
std & standard conditions \\
v & vertical direction \\
T & total \\
x & horizontal $x$ direction \\
w & water ; well \\
wc & connate water \\
z & vertical $z$ direction \\
\hline
\end{tabular}

\section{Superscripts}

$\begin{array}{ll}- & \text { average } \\ n & \text { per unit volume } \\ n & \text { time discretization level } \\ 0 & \text { related with } \Delta z_{D}=0\end{array}$




\section{References}

[1] S. Arbabi and F. J. Fayers. Comparative aspects of coning behavior in horizontal and vertical wells. In 8th European Symposium on Improved Oil Recovery, Vienna, Austria, May 15-17 1995.

[2] H. I. Meyer and A. O. Garder. Mechanics of two immiscible fluids in porous media. Journal of Applied Physics, 25(11):1400-1406, November 1954.

[3] M. Muskat. The Flow of Homogeneous Fluids Through Porous Media. Reprinted by I.H.R.D.C., Boston, 1982.

[4] D. A. Efros. Study of multiphase flow in porous media. Gastoptexizdat, Leningrad, 1963. in Russian.

[5] F. M. Giger. Evaluation théorique de l'effett d'arête d'eau sur la production par puits horizontaux. Revue de l'institut Francais du Petrole, 38(3), May 1983. In French.

[6] I. Chaperon. Theoretical study of coning toward horizontal and vertical wells in anisotropic formations: Subcritical and critical rates. In SPE 15377, 61st Annual Technical Conference and Exhibition, New Orleans, Louisiana, October 5-8 1986.

[7] M. J. Wheatley. An approximate theory of oil/water coning. In SPE 14210, 60th Annual Technical Conference and Exhibition, Las Vegas, September 22-25 1985.

[8] D. V. Addington. An approach to gas-coning corrrelations for a large grid cell reservoir simulator. JPT, pages 2267-2274, November 1981.

[9] J. Konieczek. The concept of critical rate in gas coning and its use in production forecasting. In SPE 20722, 65th Annual Technical Conference and Exhibition, New Orleans, Loisiana, September 23-26 1990.

[10] P. Papatzacos, T. R. Herring, R. Martinsen, and S. M. Skjaeveland. Cone breakthrough time for horizontal wells. SPERE, pages 311-318, August 1991.

[11] E. Ozkan and R. Raghavan. A breakthrough time correlation for coning toward horizontal wells. In SPE 20964, European Offshore Petroleum Conference, The Hague, Netherlands, October 22-24 1990. Paper SPE 20964.

[12] S. A. Tiefenthal. Supercritical production from horizontal wells in oil-rim reservoirs. SPERE, pages 272-277, November 1994.

[13] A. L. S. Souza. Correlations for cresting behavior in horizontal wells. Technical report, Ph.D. Thesis, Stanford University, 1997.

[14] Exploration Consultants Limited, UK. ECLIPSE Reference Manual Version 95a, July 1994.

[15] P. E. Gill, W. Murray, and M. H. Wright. Practical Optimization. Academic Press, San Diego, CA, 1981. 
[16] F. M. Giger. Analytic two-dimensional models of water cresting before breakthrough for horizontal wells. SPERE, pages 409-416, November 1989.

[17] W. Yang and R. A. Wattenberger. Water coning calculations for vertical and horizontal wells. In SPE 22931, 66th Annual Technical Conference and Exhibition, Dallas, October 6-9 1991. 


\title{
10. Use of Streamline Grids in Reservoir Simulation
}

\author{
by Regis Agut, Sepehr Arbabi, and Khalid Aziz
}

\begin{abstract}
Commonly used Cartesian grids should be aligned with reservoir heterogeneities to represent their effective properties. This is often difficult and sometimes impossible due to the complexity of some reservoir heterogeneity. A new numerical scheme for flexible grids has been proposed and developed by Santosh Verma [5,6]) in an Object Oriented reservoir simulator called FLEX. Different types of grids have been considered such as Voronoi, Control Volume Finite Element (CVFE), and Hexagonal grids. Streamline-based grids have also been used in FLEX but only in limited cases. Our work will consist of developing within FLEX (in $\mathrm{C}++$ ) a new grid generator based on streamlines and also on isopotential lines, in order to improve the accuracy of reservoir flow simulation in heterogeneous media.
\end{abstract}

\subsection{FLEX Simulator}

FLEX is an Object Oriented, flexible grid, black-oil reservoir simulator. The Object Oriented approach is particularly useful because of the complexity of grid generation and of flexible grid geometries (such as Voronoi, CVFE, Hexagonal grids, etc.). Further developments will therefore be made following this approach using the $\mathrm{C}++$ language.

\subsubsection{Why Flexible Grids?}

Flexible grids are made of polygons whose shape and size vary from place to place in the reservoir. Such grids have been introduced because of the limitations of the commonly used Cartesian grids and their associated numerical schemes. The types of grid and the corresponding numerical model implemented in FLEX overcome most of these limitations. Indeed, FLEX is able to align grids with :

- Reservoir boundaries

- Beds with varying permeability tensors (FLEX accepts full and asymetric tensors)

- Faults

- Horizontal/Inclined wells

FLEX can generate 2D grids based on modules of given grid-block geometry as proposed originally by Palagi [3]. Several modules can be assembled together to generate the desired grid. These modules can be scaled, rotated and then placed at any particular location (in the global coordinate system) in the reservoir using simple coordinate transformations.

In 2D, FLEX can use any one of the following modules as a base module (the modules are shown in Figure 10.1):

- Cartesian 
- Hexagonal

- Parallel Cartesian

- Tilted hexagonal

- Radial
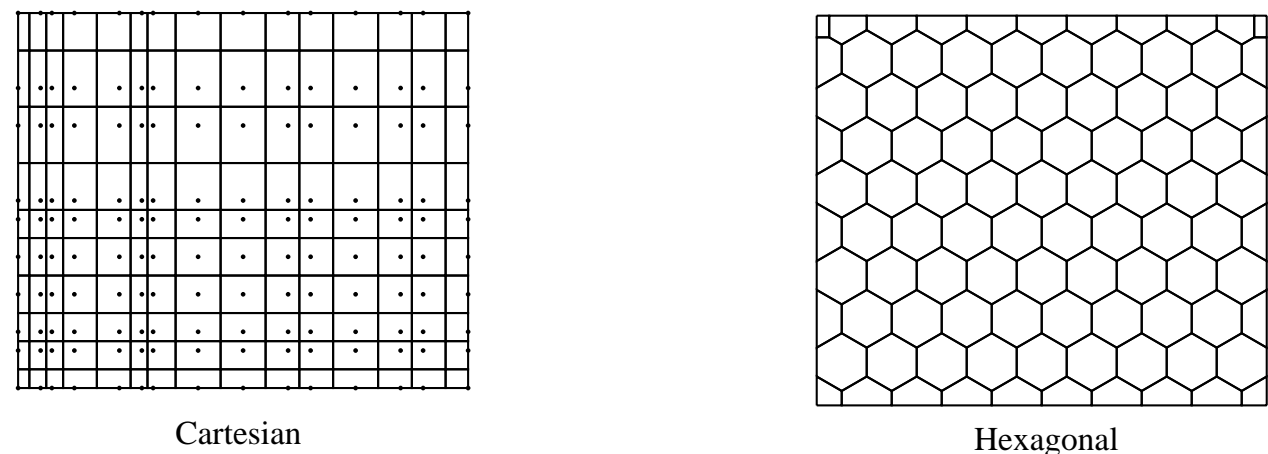

Hexagonal
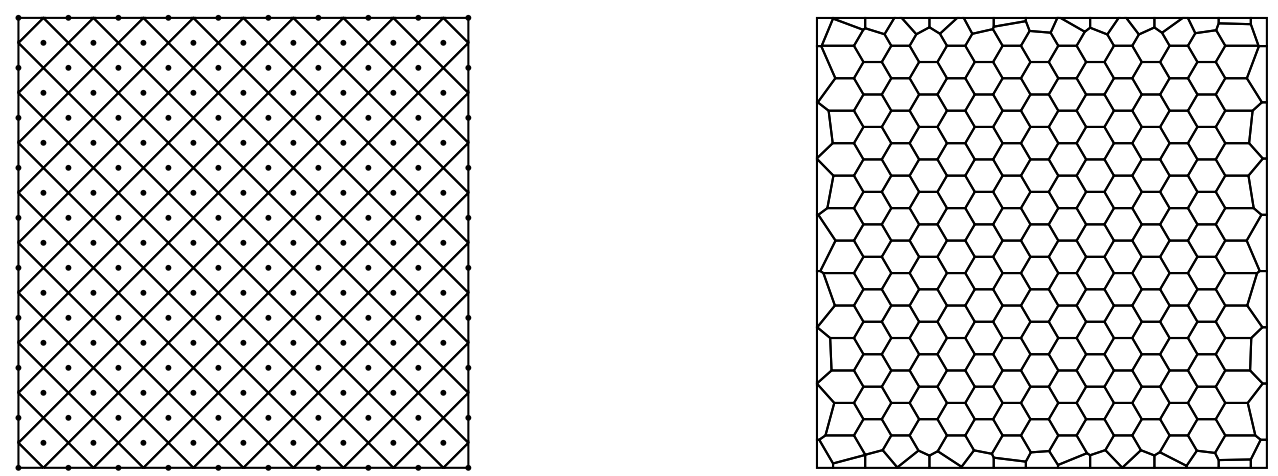

Parallel Cartesian

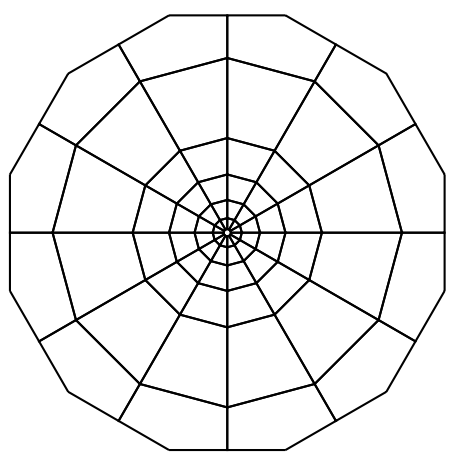

Tilted Hexagonal

Radial

Figure 10.1: Modules Used for Grid Generation in 2D (After Ref [1], Chapter 4)

Figure 10.2 shows a Voronoi grid whose grid nodes were generated using a hexagonal base grid. Radial and Cartesian modules were also placed in different regions, close to wells.

Figures 10.3 and 10.4 give other examples of Voronoi and CVFE grids. 


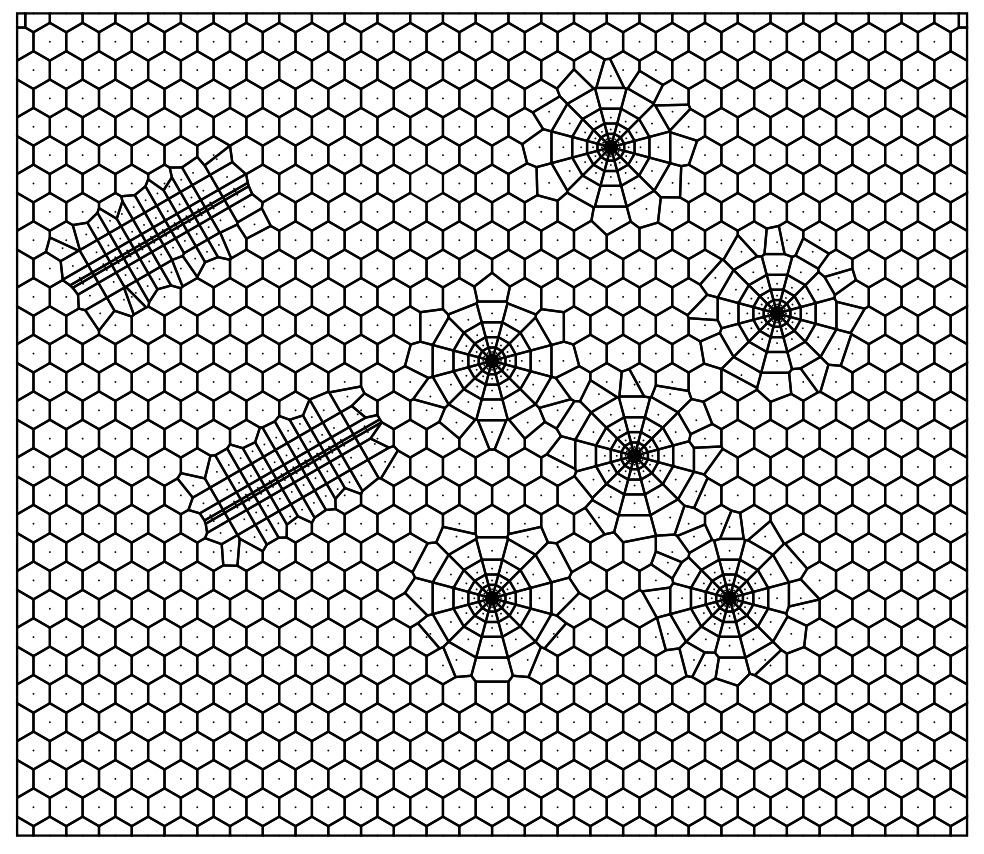

Figure 10.2: A 2D Voronoi Grid Using Modules (After Ref [1], Chapter 4) 


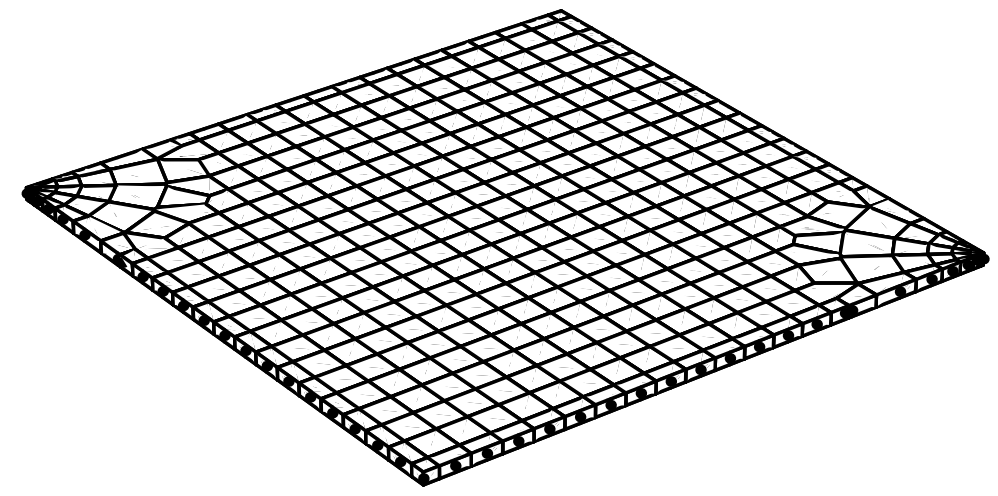

(a) X-Y Cross Section of a Point Distributed Cartesian Grid

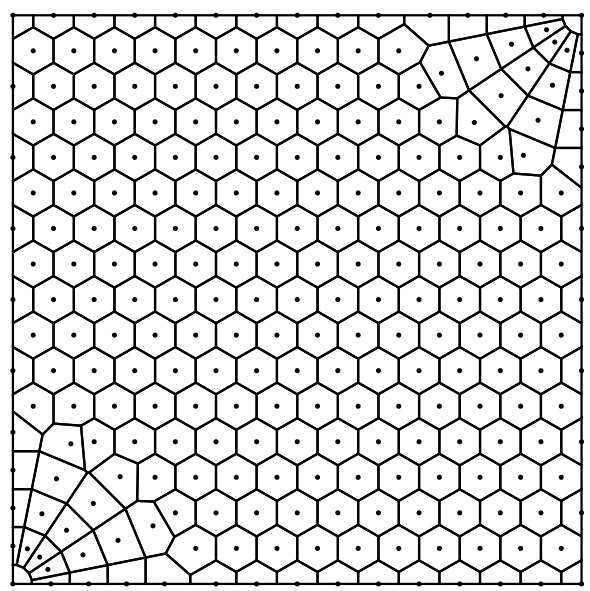

(b) 2D Hexagonal Grid

Figure 10.3: 2D Voronoi Grids with Radial Modules around Wells 


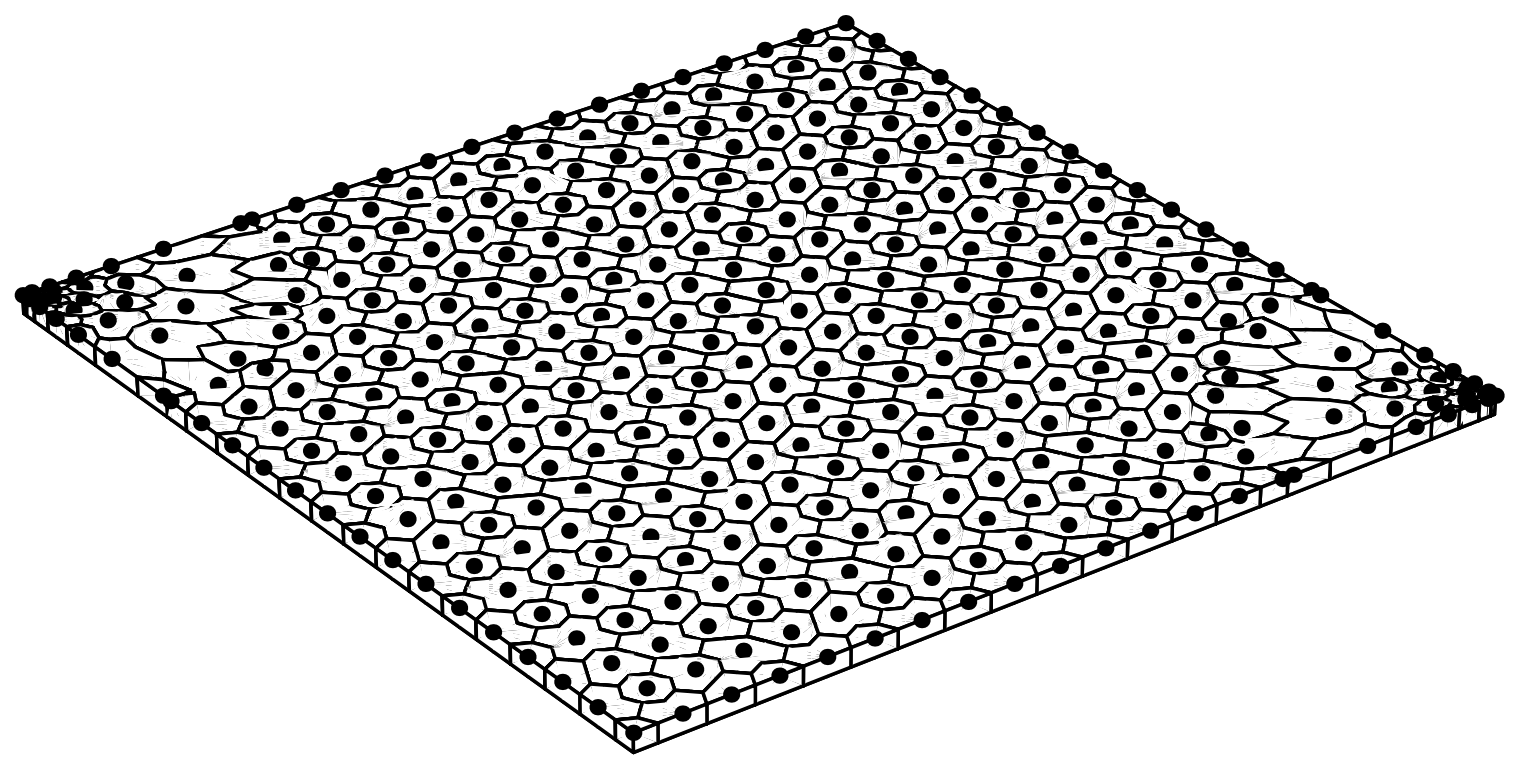

Figure 10.4: 2D CVFE Grid with Radial Modules around Wells 


\subsubsection{Numerical Model}

Grid types and assumptions about reservoir properties affect numerical properties of the solution. The mathematical formulation used to solve for the unknowns is strongly related to these assumptions.

The numerical scheme implemented in FLEX assumes that every control volume has uniform porosity and permeability. This model is based on the fact that flow at any block boundary is constrained by the velocity normal to that boundary.
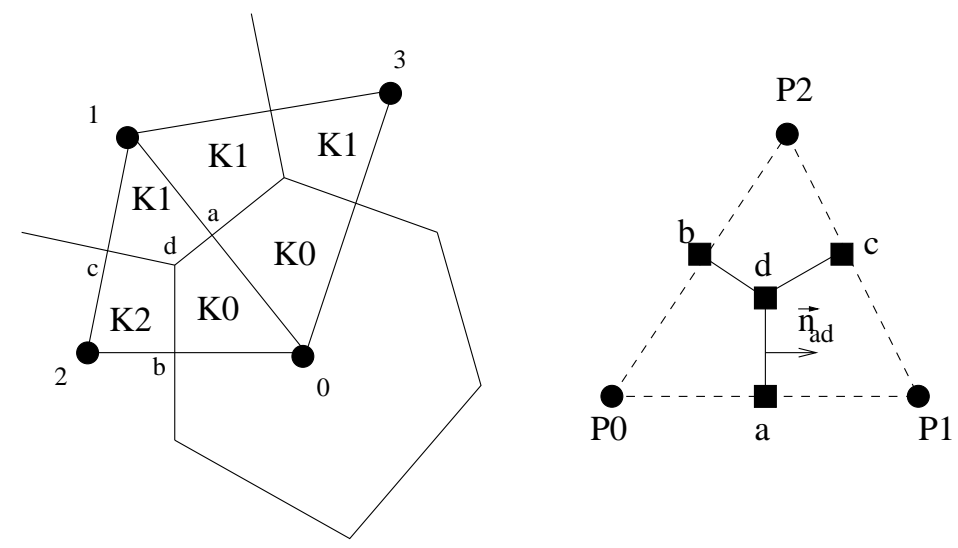

Figure 10.5: Example of a Control Volume and its Connections to the other Grid Blocks follows:

In the example of Figure 10.5, the constraint at the interface a-d can be expressed as

$$
\vec{v}_{0} \cdot \vec{n}_{a d}=\vec{v}_{1} \cdot \vec{n}_{a d}
$$

where $\vec{v}_{i}$ is the velocity in region $i(i=0,1), \vec{n}_{a d}$ is the outward normal on the interface a-d.

The potential at the nodes are obtained by an interpolation between potentials at the interfaces. The flow term corresponding to the nodes considered is :

$$
q_{0 a}=-\int_{a}^{d} \vec{v}_{0} \cdot \vec{n}_{a d} d L=-A_{a d} \vec{v}_{0} \cdot \vec{n}_{a d}
$$

where $A_{a d}$ is the length of the interface a-d and this becomes a surface in 3D.

Substituting for the velocity using Darcy's law: $\overrightarrow{v_{0}}=-\left[\overline{\bar{k}}_{0} \cdot \vec{\nabla} \Phi_{0}\right]$ and solving for the face potentials in terms of the node potentials, the constraint equation can be written in the following form :

$$
\left[\begin{array}{l}
\Phi_{a} \\
\Phi_{b} \\
\Phi_{c}
\end{array}\right]=\left[\begin{array}{lll}
R_{00} & R_{01} & R_{02} \\
R_{10} & R_{11} & R_{12} \\
R_{20} & R_{21} & R_{22}
\end{array}\right]\left[\begin{array}{l}
\Phi_{0} \\
\Phi_{1} \\
\Phi_{2}
\end{array}\right]
$$

The flow terms can thus be expressed as follows:

$$
\left[\begin{array}{c}
q_{a} \\
q_{b} \\
q_{c}
\end{array}\right]=\underbrace{\left[\begin{array}{ccc}
T_{a 0} & T_{a 1} & T_{a 2} \\
T_{b 0} & T_{b 1} & T_{b 2} \\
T_{c 0} & T_{c 1} & T_{c 2}
\end{array}\right]}_{T}\left[\begin{array}{c}
\Phi_{0} \\
\Phi_{1} \\
\Phi_{2}
\end{array}\right]
$$


$T$ is the transmissibility matrix for node 0 which represents the contribution of the triangle $\Delta_{012}$.

All the flow terms for a given node $i$ for each triangle (tetrahedron in 3D) are then collected and the conservation equation for the node is put in the following form :

$$
\sum_{j=1}^{n_{c}}\left[\sum_{k=1}^{n_{s c}}\left(\sum_{l=1}^{n_{s c, l} l} T_{k l} \Phi_{l}\right)\right]_{i}=\underbrace{\frac{V_{i}}{\Delta t}\left[\left(\frac{\Phi S_{p}}{B_{p}}\right)^{n+1}-\left(\frac{\Phi S_{p}}{B_{p}}\right)^{n}\right]_{i}}_{\text {Accumulation Term }}
$$

where $n_{c}$ is the number of connections for a grid node, $n_{s c}$ is the number of subconnections of a connection and $n_{s c, l}$ is the number of nodes which affect flow for a subconnection.

\subsection{Use of Streamline Grids in Simulation}

Streamlines are being used more and more extensively in reservoir flow simulation to characterize flow patterns (Thiele et al. 1996 [4]). Indeed, this is a very efficient way to deal with displacement processes governed by reservoir heterogeneities.

S.Verma and M.Thiele proposed a method to generate a grid based on streamlines. This has been implemented in FLEX but with the assumption of approximating the isopotential lines by straight lines. Figure 10.6 shows the streamlines for an example in 2D (XY) where the wells are located at both ends of the reservoir. The injector is at the left boundary and the producer at the right boundary.

The streamlines are arbitrarily selected at equal intervals and grid-blocks are con-

strained only to the selected streamlines. A coarser streamline-based grid is then generated (See Figure 10.7). 


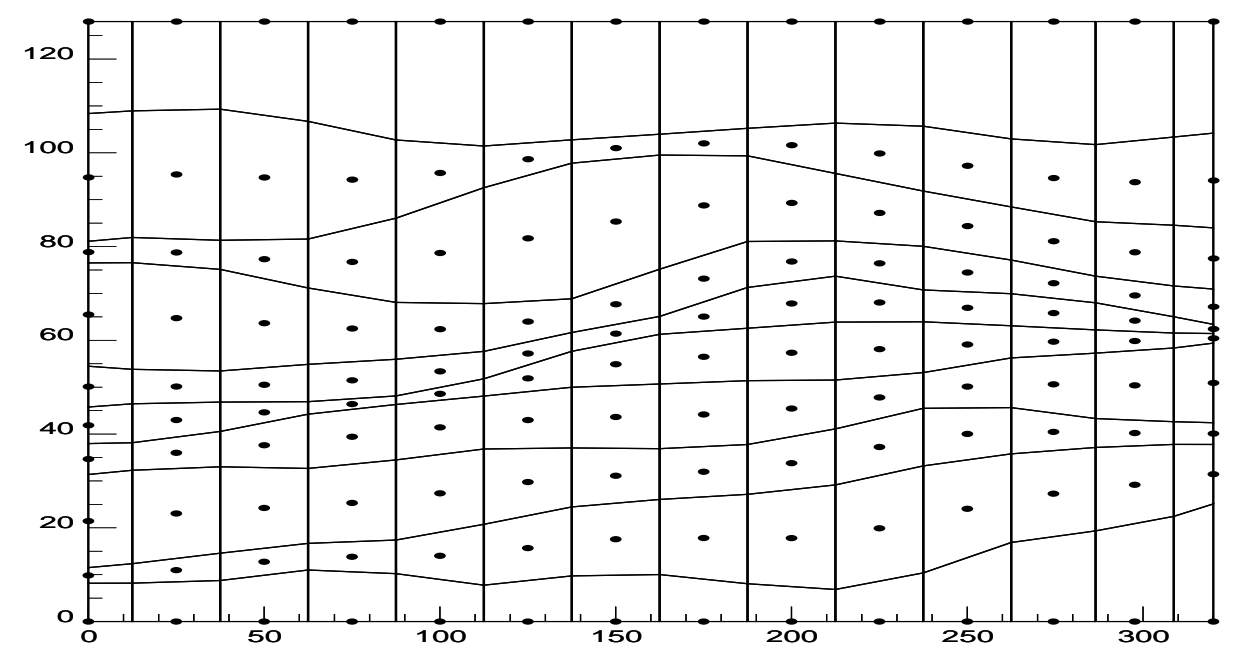

Figure 10.6: Streamline Grid Generated after Selecting Some Streamlines and Upscaling Permeabilities

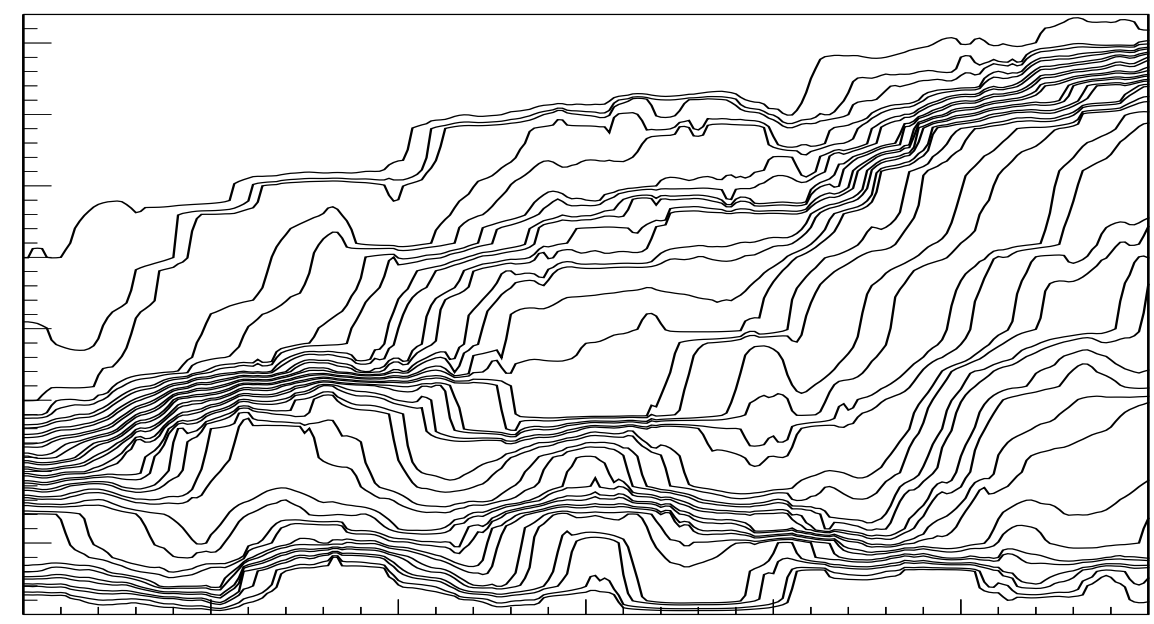

Figure 10.7: Streamlines generated by Injecting at the Most Left Boundary and Producing at the Most Right Boundary 
It was observed that such a grid (aligned to streamlines) significantly improves the water-cut response when compared with a Cartesian grid with the same number of grid nodes.

\subsubsection{New Work on streamline grids}

A new grid generation algorithm is being implemented in FLEX in an object oriented framework (in $\mathrm{C}++$ ). The new grid will be defined by streamlines and equipotentials. These grids are of interest because they reflect variations in the pressure field caused by permeability heterogeneities in the reservoir and can cluster grid lines in high flow regions. This should increase local accuracy of the finite-volume discretization.

First, the streamlines are generated (using for example a streamline-based simulator). The isopotential lines are then calculated using an interpolation algorithm on the pressure values along the streamlines. The intersections between streamlines and isopotential lines are calculated. These intersections correspond to the vertices of the control volumes. Therefore, every control volume is a quadrilateral. The grid nodes are placed in the center of each control volume. This center corresponds to the intersection of the segments joining the mid-points of opposite faces (See Figure 8).

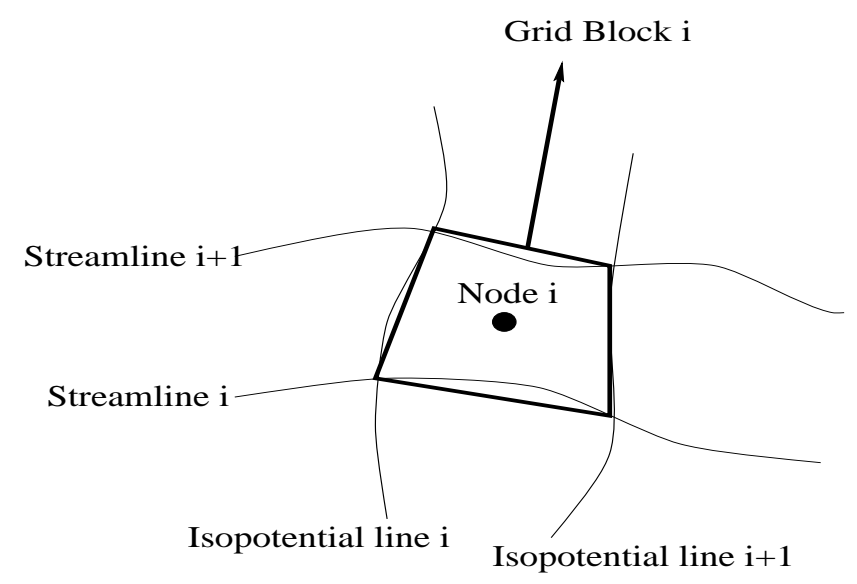

Figure 10.8: Construction of Grid Blocks Using Streamlines and Equipotentials

Once the nodes and the control volumes are generated, we can create the connections between nodes and then calculate the transmissibilities for all the connections.

Flux continuity is imposed following Edwards and Rogers 1994 [2], using the same approach as the one used for the triangles (Verma and Aziz 1996 [6]). The numerical scheme is the same but instead of triangular connections, we have a quadrilateral. The potential and velocity continuity points are located as shown in Figure 9:

Using the same approach as in Section 2.2 on triangles, we can express the flow continuity for the node 0 by the following constraint :

$$
\vec{v}_{0} \cdot \vec{n}_{a}=\vec{v}_{1} \cdot \vec{n}_{a}
$$

Then, we obtain the potential at the interfaces as a linear function of the potentials at the nodes and put it in the following matrix form : 


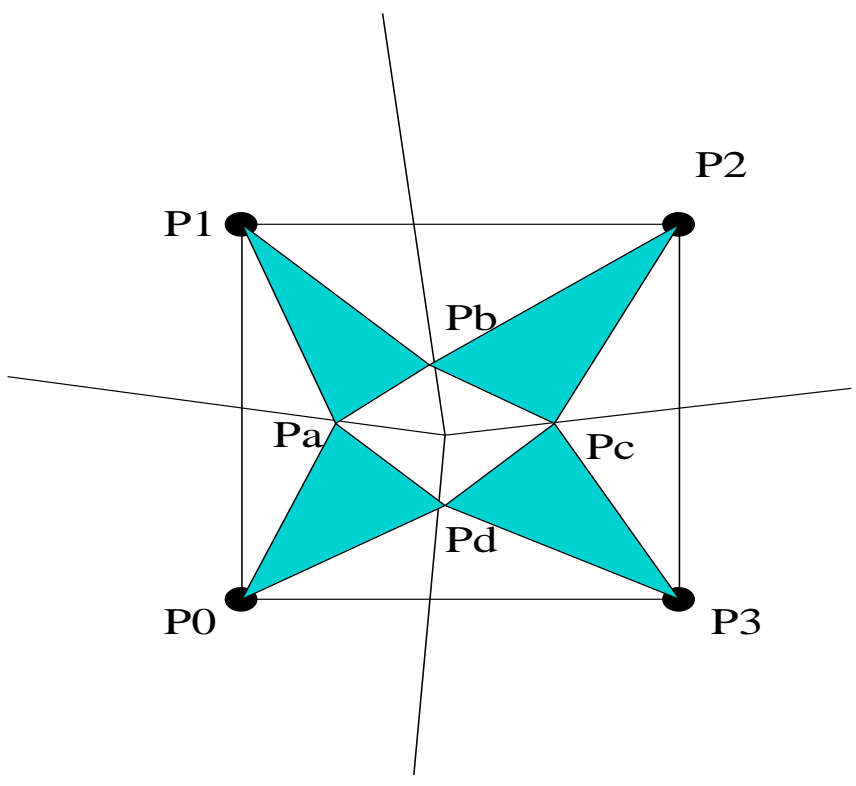

Figure 10.9: Locations of Pressure Points for Interpolation and Transmissibility Calculations

$$
\left[\begin{array}{l}
\Phi_{a} \\
\Phi_{b} \\
\Phi_{c} \\
\Phi_{d}
\end{array}\right]=\left[\begin{array}{llll}
R_{00} & R_{01} & R_{02} & R_{03} \\
R_{10} & R_{11} & R_{12} & R_{13} \\
R_{20} & R_{21} & R_{22} & R_{33} \\
R_{30} & R_{31} & R_{32} & R_{33}
\end{array}\right]\left[\begin{array}{c}
\Phi_{0} \\
\Phi_{1} \\
\Phi_{2} \\
\Phi_{3}
\end{array}\right]
$$

This method allows the points of continuity $P_{a}, P_{b}, P_{c}$ and $P_{d}$ to be chosen along their corresponding segments, using a factor $\alpha$.

$$
\alpha \in] 0 ; 1[\text { and } \alpha=0.5 \text { gives the mid-point. }
$$

Figure 10.10 shows an example of a grid generated by this process:

\subsubsection{Results}

Grids generated with streamlines and isopotential lines are now being tested in order to test for improvements in the accuracy of such an approach, compared to classical Cartesian grids. Some results are shown in this section. The first example considered here corresponds to the permeability field shown on the top of the Figure 10.11. This fine grid (256x128) has been upscaled using a flow-based upscaling method (Figure 10.12). As shown by Figure 10.13, the grid is not very far from a Cartesian grid because there are no big distorsions on the distribution of permeabilities. The water-cut response given in figure 10.14 shows that cartesian grid and streamline grids give very close results. Nevertheless, we observe that the more the grid is aligned along heterogeneities, higher the water-cut at early time. This is the effect of taking into account the flow-paths to build the grid.

The second example is based on the permeability field shown in Figure 10.15 (CASE 2 ). We can see important differences between the cartesian grid and the one built with streamlines and isopotential lines (Figure 10.16). The impact of this grid on the water-cut performance is shown in Figure 10.17 


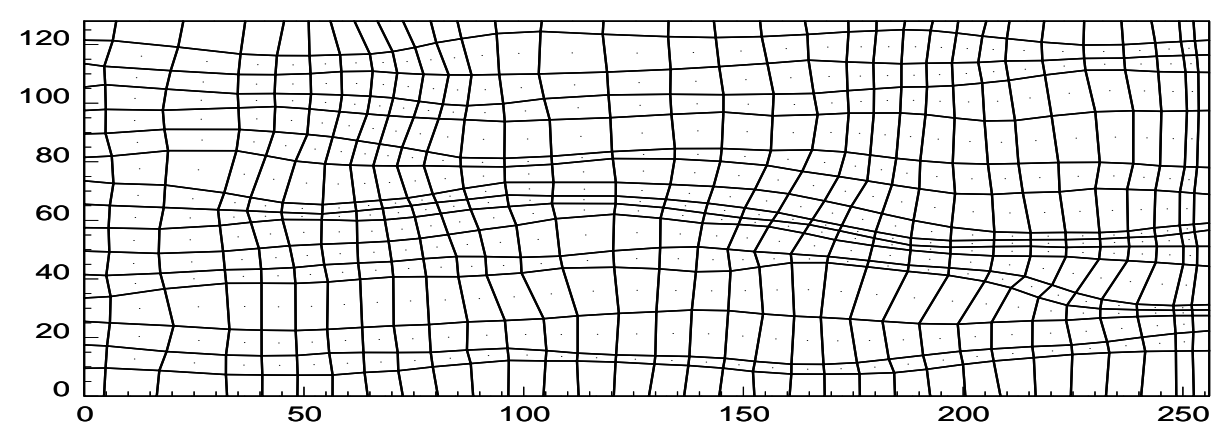

Figure 10.10: Streamline/Isopotential Line Grid
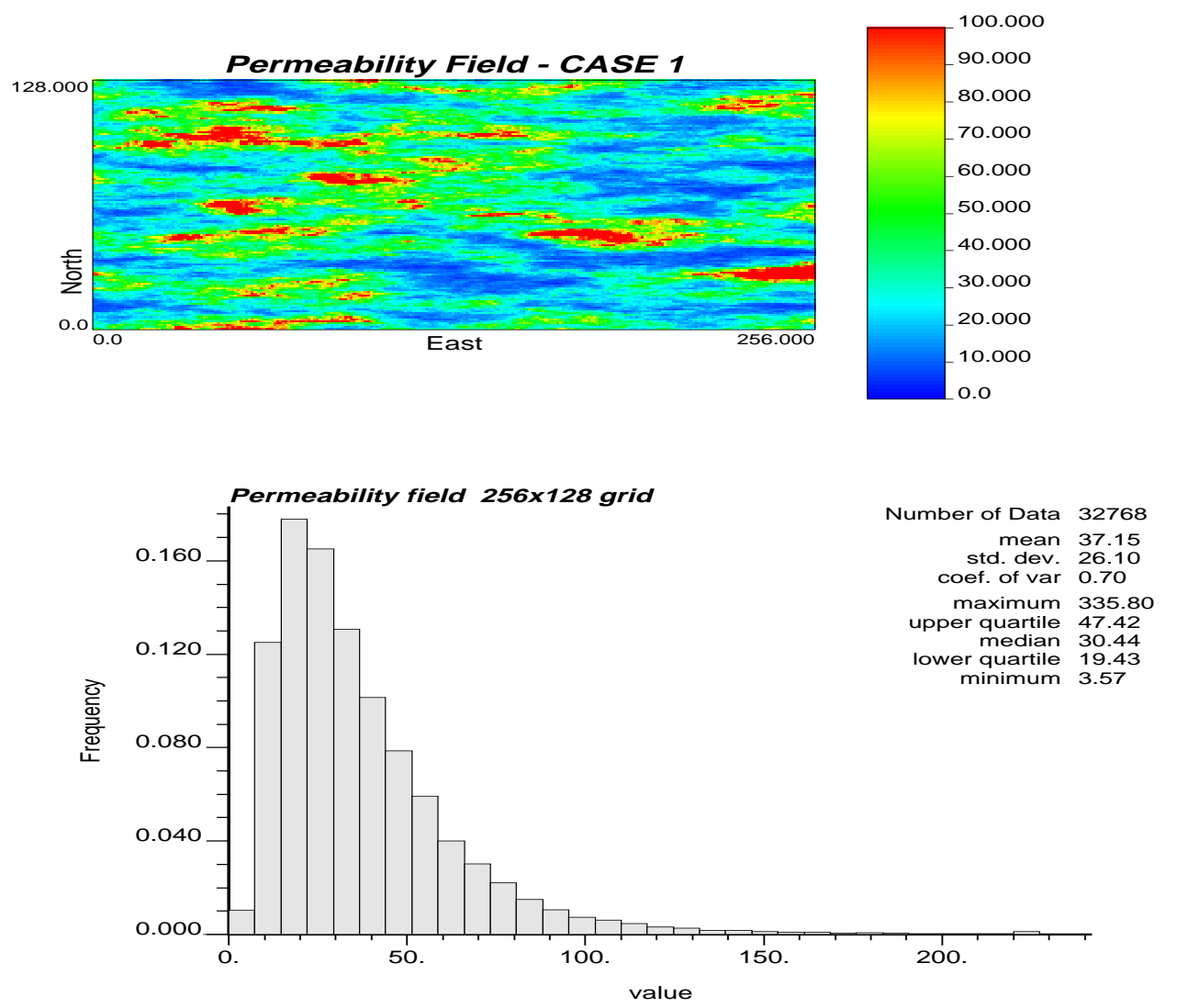

Figure 10.11: Permeability Field 1 

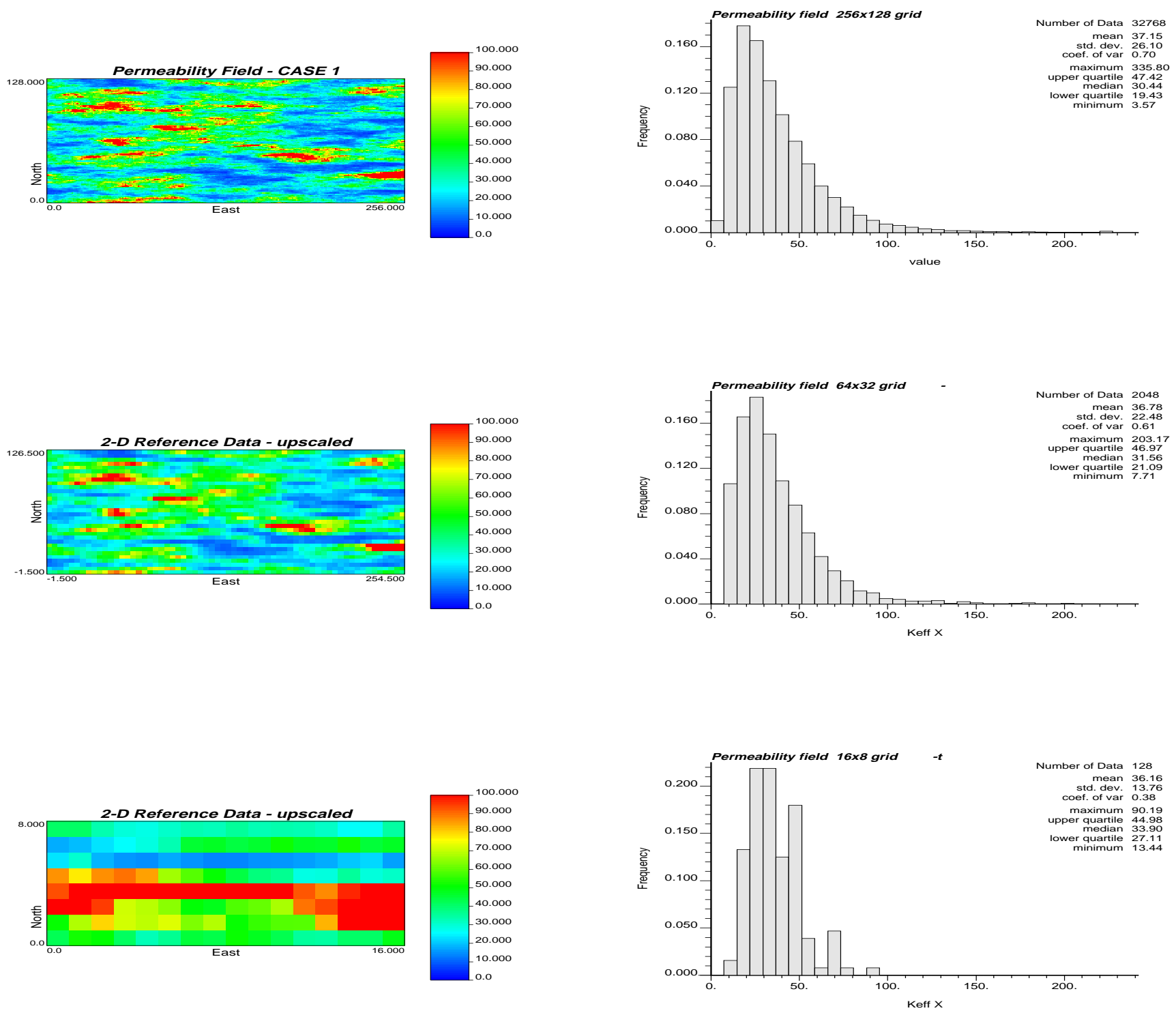

Figure 10.12: Permeability Field 1 

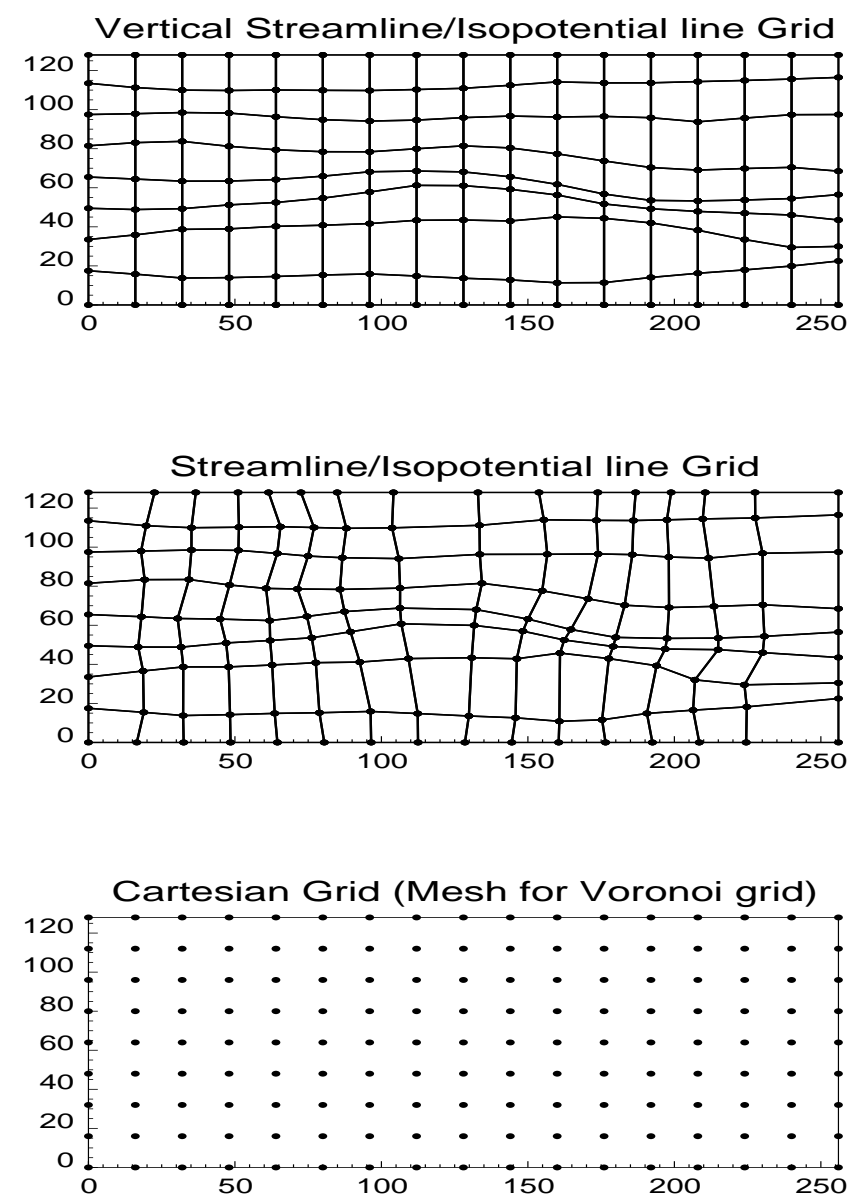

Figure 10.13: Three Different Grids Corresponding to the Permeability Field 1 


\section{WATER CUT : Case 1 \\ (Upscaling : Power Law average $w=0.5$ )}

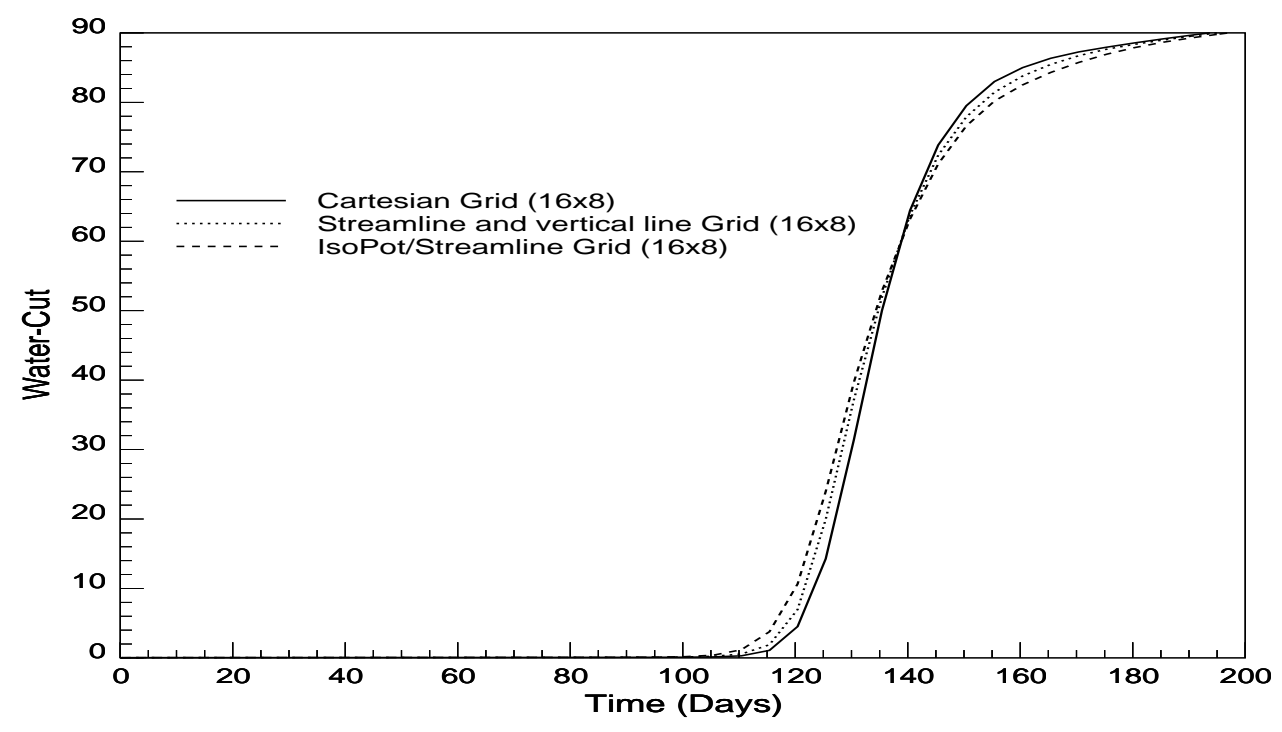

Figure 10.14: Water-Cut Response for the Three Grids Used in CASE 1
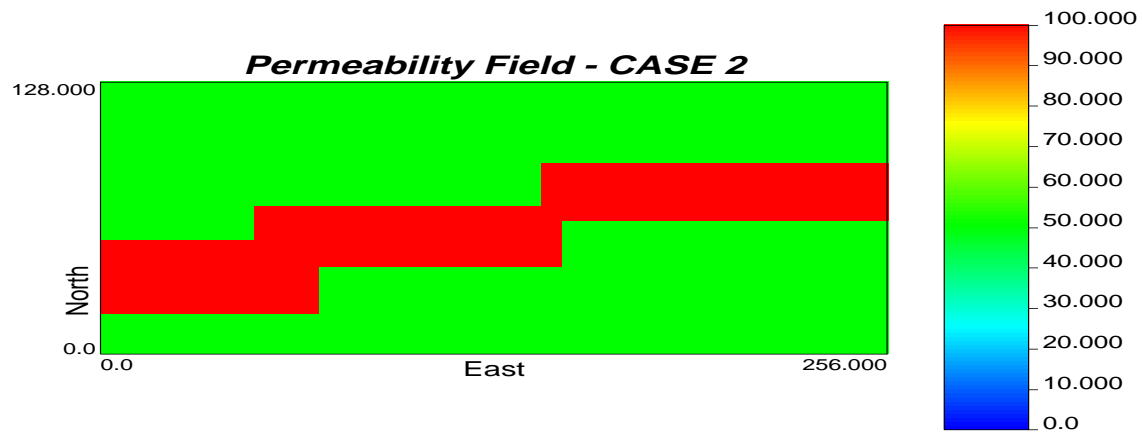

Figure 10.15: Permeability Field 1 
Streamline/Vertical line Grid

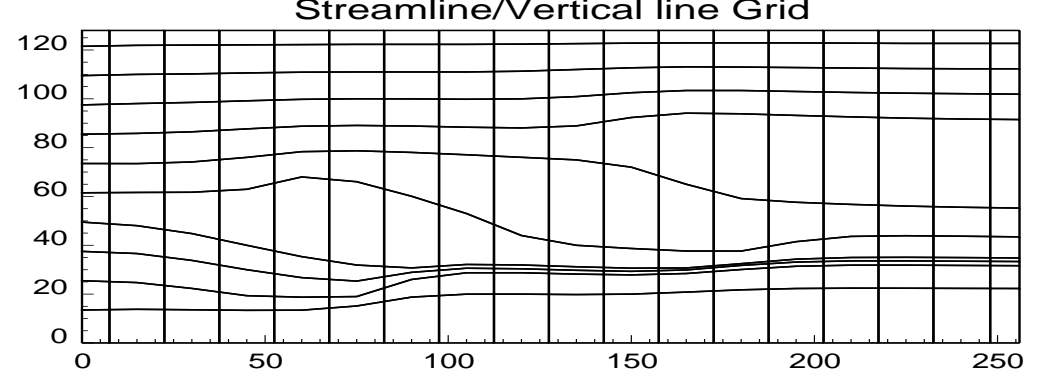

Streamline/Isopotential line Grid

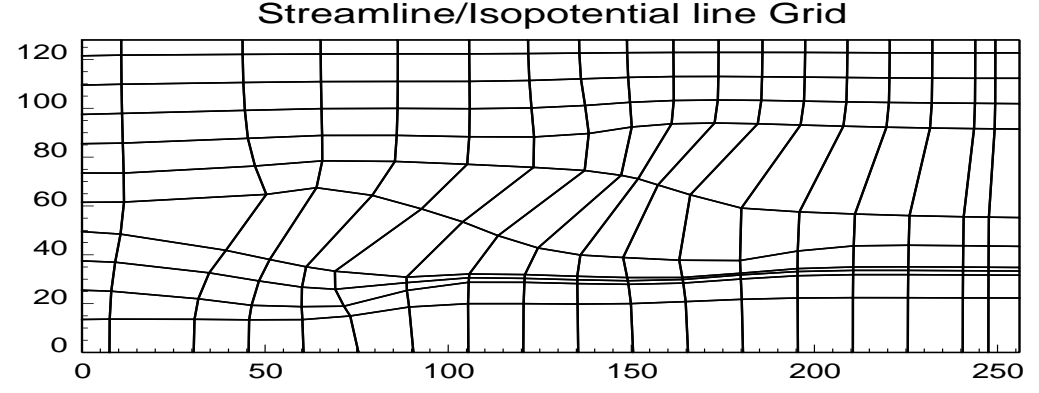

Cartesian Grid (Mesh for Voronoi grid)

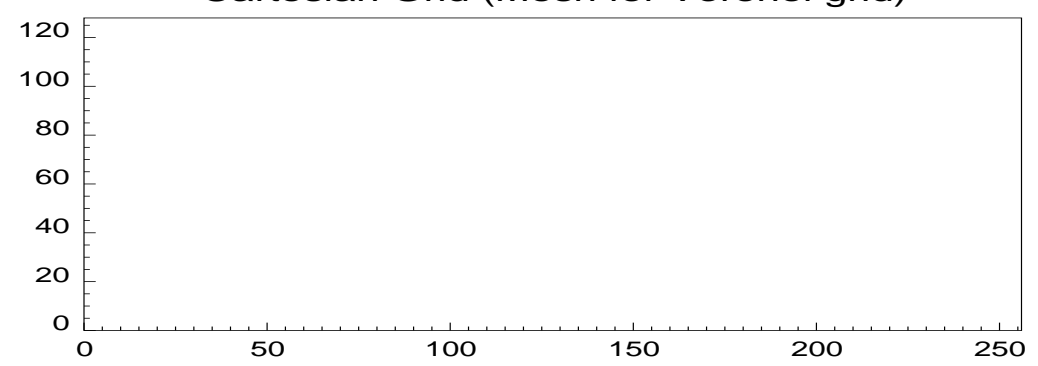

Figure 10.16: Three Different Grids Corresponding to the CASE 2 


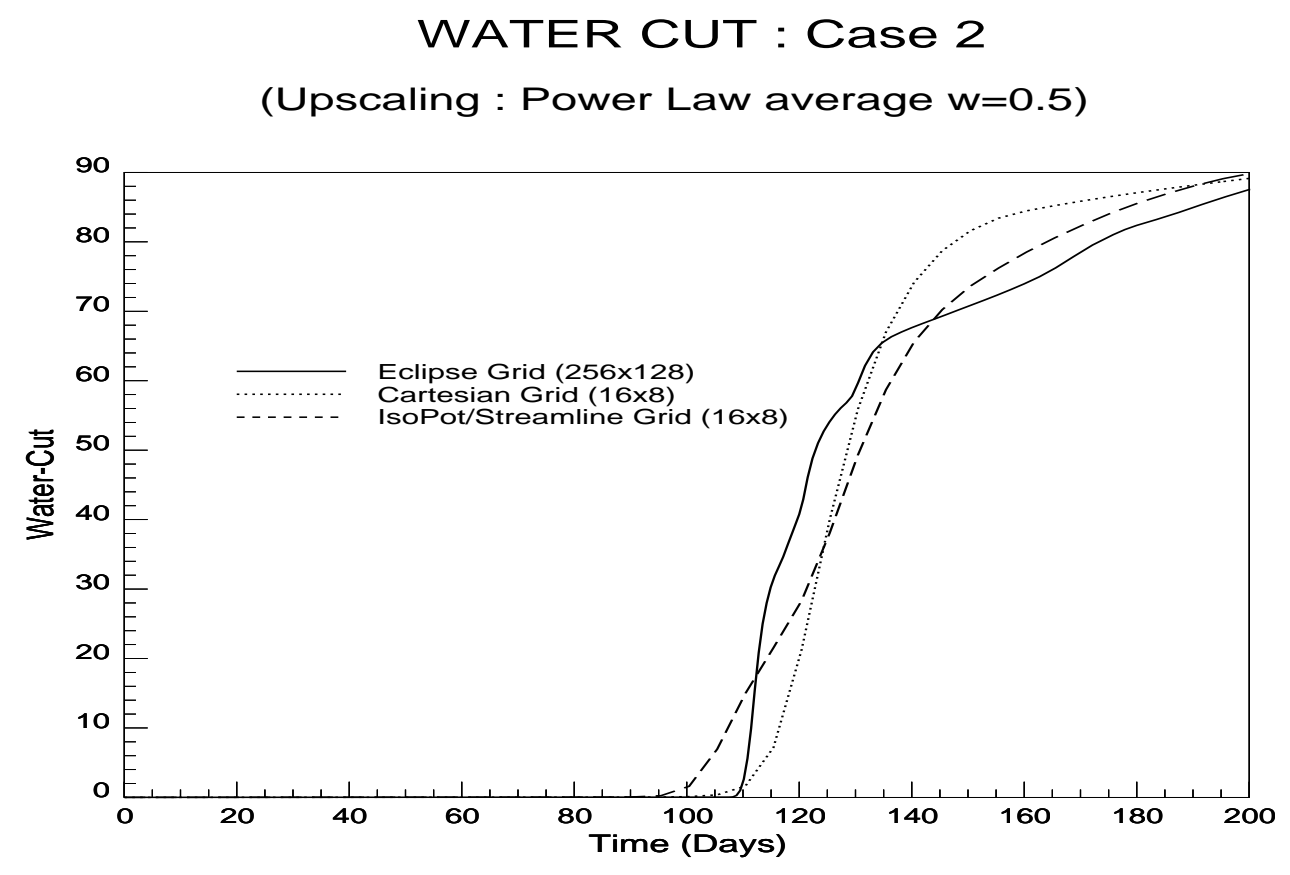

Figure 10.17: Water-Cut Response for the Three Grids Used in CASE 2 


\subsubsection{Future Work}

Future work will involve the extension of the streamline generator (in 2-D first and then in 3-D) to arbitrary well configurations and application to:

- Compressible flow

- Flow with diffusive/dispersive components added to the total flow, like tranverse diffusion and tranverse capillary pressure

- Gravity dominated flow (this problem with mild gravity has just recently been dealt with by operator splitting in a streamline simulator)

A longer term goal is to couple a streamline simulator (such as 3DSL [1,4]) with FLEX. This would yield essential information on flow paths and help to generate realistic grids to be used by FLEX which can handle complex grid geometries in 2D and 3D. Periodic updates of the grid may also be considered. Currently, effects of upscaling and grid level are being investigated compared to time grid simulations.

\section{Acknowledgements}

We would like to thank Santosh Verma for the time he spent on various aspects of this project. RA is especially grateful for his time and technical support.

Valuable discussions with Marco Thiele and Michael Edwards are also gratefully acknowledged. 


\section{References}

[1] Batycky, R.P.:"Field Scale 3-D Streamline Simulator - User Manual," Stanford University, January, 1997.

[2] Edwards, M.G. and Rogers, C.F.:"A Flux Continuous Scheme for the Full Tensor Pressure Equation," Proceedings: 4th European Conference on the Mathematics of Oil Recovery, Norway 1994.

[3] Palagi, C.L.: "Generation and Application of Voronoi Grids to Model Flow in Heterogeneous Reservoirs," Ph.D. Dissertation, Stanford University,May 1992.

[4] Thiele, M.R.., Batycky, R.P., Blunt, M.J. and Orr, F.M.: "Simulating Flow in Heterogeneous Systems Using Streamtubes and Streamlines," SPERE (February 1996), 11, No. 1, 5-12.

[5] Verma, S.: "Flexible Grids for Reservoir Simulation," Ph.D. Dissertation, Stanford University, 1996.

[6] Verma, S., Aziz K.: " Two- and Three-Dimensional Flexible Grids in Reservoir Simulation," paper presented at the 5th European Conference on Mathematics of Oil Recovery, Loeben, Austria, 3-6 Sept, 1996. 